The Biosynathesis of $A$ mplnotericin $\mathbb{B}$

Thesis submitted for the degree of

Doctor of Phillosophy

by

Carmell Marie McNamara

Department of Chemistry

University of Leicester

September 1996 
UMI Number: U087475

All rights reserved

\section{INFORMATION TO ALL USERS}

The quality of this reproduction is dependent upon the quality of the copy submitted.

In the unlikely event that the author did not send a complete manuscript and there are missing pages, these will be noted. Also, if material had to be removed, a note will indicate the deletion.

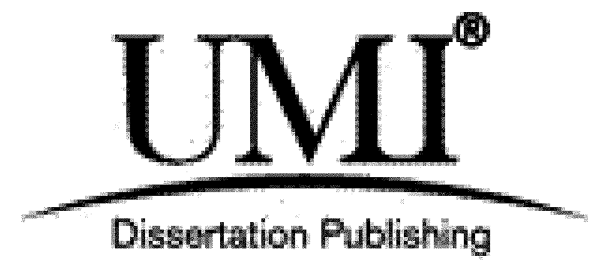

UMI U087475

Published by ProQuest LLC 2015. Copyright in the Dissertation held by the Author.

Microform Edition (c) ProQuest LLC.

All rights reserved. This work is protected against unauthorized copying under Title 17, United States Code.

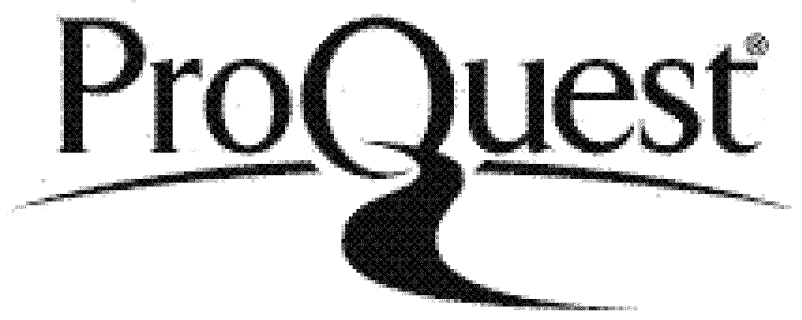

ProQuest LLC

789 East Eisenhower Parkway

P.O. Box 1346

Ann Arbor, MI 48106-1346 


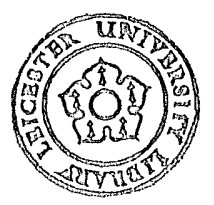




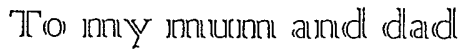




\title{
BIOSYNTHESIS OF AMPHOTERICIN B
}

\author{
Carmel Marie McNamara
}

\begin{abstract}
The polyene antibiotic and natural product amphotericin $\mathrm{B}$ is produced by Streptomyces nodosus, together with its co-metabolite amphotericin A. Polyene antibiotics are used in the treatment of fungal diseases, despite their potent human toxicity. Information on the biosynthetic pathway would provide us with the basic information to produce less toxic analogues. An investigation into the biosynthesis of amphotericin B was undertaken which involved a variety of methods and techniques. Attempted incorporation of precursors into the metabolite was a major part of this work, with the feeding of $\left[{ }^{13} \mathrm{C}_{1}\right]$ - and $\left[{ }^{13} \mathrm{C}_{2}\right]$-acetates and diethyl $\left[{ }^{13} \mathrm{C}_{1}\right]$ malonate, with any incorporation being detected by NMR and mass spectrometry. The synthesis of the deuteriated $(2 S, 3 S)-3$-hydroxy-2- $\left[{ }^{13} \mathrm{C}_{3}\right]$ methyl[3${ }^{13} \mathrm{C}$ ]butanoyl $\mathrm{N}$-acetylcysteamine thioester was undertaken, which was consequently fed to cultures of $S$. nodosus. An investigation of growth and production of amphotericin B from cultures of $S$. nodosus was carried out in order to determine the optimum time for the feeding of precursors. An examination of extracts of the culture broth also provided information on co-metabolites and possible putative intermediates present, which, although only a brief introductory study, indicated the potential importance of such investigations. Some evidence for the proposed mode of biosynthesis of amphotericin B was found, although there was no detection of incorporation of the administered deuteriated thioester. The background work into the growth of Streptomyces nodosus and production of amphotericin B that was carried out in this study will form the basis for any future investigations into the biosynthesis of this important metabolite.
\end{abstract}




\section{Acknow ledgements}

I would like to thank my supervisor, Dr Bernard Rawlings, for his encouragement, enthusiasm and guidance throughout my $\mathrm{PhD}$. Thanks to my industrial supervisor, Steve Box, who was always ready with helpful suggestions and willing to put up with me taking over the equipment at the Pilot Plant. Thanks also to Mike Rees, Jo, Steve and everyone else at Brockham Park who made my visits so enjoyable.

In the lab, I would like to thank Mick Lee for his technical expertise, Dr Graham Eaton for providing the mass spec service and Dr Gerry Griffiths for running my NMR spectra. Thanks very much to everyone who made my life in the lab so enjoyable, and I hope you think of me when you find yourself singing the 'Heidi' theme-tune.

Special thanks go to Phill for being a good friend and for all the excellent mapdrawing on his fume-hood, and to Jim for having someone else to blame for the smell that came wafting up from the culture prep room when we were playing with our bugs.

Whilst writing up, I would like to thank Deanne for keeping me fed and watered when I was too busy to do so myself, and special thanks to Simon for helping me see the lighter side of life when it was all getting me down.

Finally I would like to thank my family, without whose love, support and encouragement, I wouldn't have made it this far.

Is fáda an lá ná dtiochfainn an óiche. 


\section{Statement}

The accompanying thesis for the degree of Ph.D. entitled The biosynthesis of amphotericin $B$ is based on work conducted by the author in the Department of Chemistry of the University of Leicester during the period October 1992 to September 1995. Unless otherwise acknowledged in the text or by reference, all the work recorded herein is original to the best of my knowledge. None of the work has been submitted for another degree in this or any other university.

Signed Goud Ysaygh Date 28 rod April 1997 


\section{Abbreviations}

$\begin{array}{ll}\text { ACP } & \text { Acyl carrier protein } \\ \text { AmA } & \text { Amphotericin A } \\ \text { AmB } & \text { Amphotericin B } \\ \text { BuLi } & \text { Butyllithium } \\ \text { CM } & \text { Complex media } \\ \text { CoA } & \text { Coenzyme A } \\ \text { DCC } & \text { Dicyclohexylcarbodiimide } \\ \text { DEPT } & \text { Distortionless enhancement by polarisation transfer } \\ \text { DIP } & \text { Diisopropylamine } \\ \text { DM } & \text { Defined media } \\ \text { DMAP } & \text { Dimethyaminopyridine } \\ \text { DMCD } & \text { Dimethylcyclodextrin } \\ \text { DMF } & N, N \text {-Dimethyformamide } \\ \text { DMPU } & \text { 1,3-Dimethyl-3,4,5,6-tetrahydro-2(1H)-pyrimidinone } \\ \text { DMSO } & \text { Dimethylsulfoxide } \\ \text { DNA } & \text { Deoxyribonucleic acid } \\ \text { EI } & \text { Electron impact } \\ \text { ESMS } & \text { Electrospray mass spectrometry } \\ \text { FAS } & \text { Fatty acid synthase } \\ \text { FCA } & \text { Fructose-collofilm dextrin-arkasoy } \\ \text { GYE } & \text { Glucose-yeast extract } \\ \text { HMPA } & \text { Hexamethylphosphoramide } \\ \text { HPLC } & \text { High performance liquid chromatography magnetic resonance } \\ \text { LCMS } & \text { Liquid chromatography mass spectrometry } \\ \text { LDA } & \text { Lithium diisopropylamine } \\ \text { NAC } & \text { NMR }\end{array}$


ORF

4-PA

PKS

RNA

SF

3-TDPA

THF

TLC
Open reading frame

4-Pentynoic acid

Polyketide synthase

Ribonucleic acid

Shake flask

3-(Tetradecylthio)propanoic acid

Tetrahydrofuran

Thin layer chromatography 


\section{Compternts}

\section{Chapter 1 Introduction}

1.1 Background 1

1.2 Evolution of polyketides 5

1.2.1 History 5

1.2.2 Fatty acid biosynthesis 6

1.3 Polyketide biosynthesis 9

1.3.1 Aromatic polyketides 11

1.3.2 Non-aromatic polyketides 11

1.3.2.1 Processive $v s$. non-processive hypothesis 16

1.3.2.2 Evidence for processive hypothesis 21

1.3.2.3 Further evidence: genetic analysis $\quad 29$

1.4 Amphotericin B 33

1.4.1 Background $\quad 33$

1.4.2 Mode of action $\quad 35$

$\begin{array}{ll}\text { 1.4.3 Biosynthesis } & 36\end{array}$

\section{Chapter 2 Synthesis of target labelled diketide}

2.1 Introduction 39

2.2 Synthetic strategy $\quad 42$

2.2.1 Approaches 42 
2.2.2.1 Methodology: $(S)$-Valine route

2.2.2.2 (R)-Valine route and incorporation of labels

\section{Chapter 3 Biological studies}

3.1 Introduction $\quad 89$

3.2 Growth and production 90

$\begin{array}{ll}3.2 .1 \text { General } & 90\end{array}$

3.2.2 Amphotericin B 94

3.2.2.1 Whole broth production 97

3.2.2.2 Resuspension of cells 105

$\begin{array}{ll}3.3 \text { Extraction and purification } & 114\end{array}$

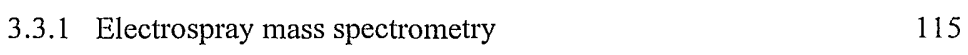

3.3.2 NMR spectroscopy 125

3.4 Search for minor co-metabolites of amphotericin 128

\section{Chapter 4 Labelling studies}

4.1 Introduction 142

4.2 Acetate feedings 144

4.2.1 $\left[1,2-{ }^{13} \mathrm{C}_{2}\right]$ Acetate feeding $\quad 147$

4.2.2 $\left[1-{ }^{13} \mathrm{C}\right]$ Acetate and $\left[2{ }^{13} \mathrm{C}\right]$ acetate feeding 160

4.2.3 $\left[1-{ }^{13} \mathrm{C}\right]$ Acetate and diethyl $\left[2-{ }^{13} \mathrm{C}\right]$ malonate feeding 162

4.3 Diketide feeding 166

Chapter $\mathbf{5}$ Summary and future studies $\quad 178$

$\begin{array}{lll}5.1 \text { Summary } & 178\end{array}$

$\begin{array}{lll}5.2 & \text { Future studies } & 180\end{array}$ 


\section{Chapter 6 Experimental}

$\begin{array}{lll}6.1 \text { General } & 181\end{array}$

6.1.1 Materials 181

6.1.2 Solvents 181

6.1.3 Methods and instrumentation $\quad 182$

6.2 Chemical experimental details 184

6.3 Biological experimental details 213

6.3.1 Composition of culture media 213

6.3.2 Growth of Streptomyces nodosus 215

6.3.3 Quantitative determination of amphotericin $\mathrm{A}$ and amphotericin B concentration 216

6.3.4 Extraction and purification $\quad 216$

6.3.5 Addition of labelled compounds to the fermentation broth 221 


\section{Chappere 1}

\section{Hmitroduction}

\subsection{Baclkground}

Throughout history, natural products have been an integral part of our every day lives. Their applications vary widely, from stimulants (caffeine 1) and narcotics (morphine 2) to a whole host of medicines (e.g. ephedrine 3, ricinine 4 and quinine 5; Fig. 1.1). Although many natural products can be beneficial to either man or the microorganism in the situations to which they are applied, many may serve no apparent useful purpose to the microorganisms that produce them.

Natural products can be divided into two main categories, primary and secondary metabolites. All organisms, from bacteria to plants and mammals, produce (synthesise and utilise) chemicals that are essential for their own general well being, growth and survival, for example amino acids, sugars, nucleotides and derived polymers (RNA, DNA etc.), which are known as primary metabolites.' The secondary metabolites are the class of natural products that appear to have little use in the 'day to day' life of the producing organism, and more frequently only produced by bacteria, fungi, plants and some insects. The pathways that produce 
Stimulants<smiles>O=c1[nH]c(=O)c2c(ncn2[Na])[nH]1</smiles>

Caffeine 1

Tea, coffee plants
Narcotics

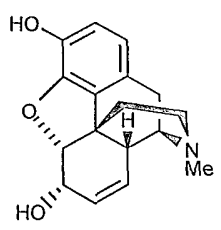

Morphine 2 (Opium)

Papaver sominiferum L.

\section{Medicines}

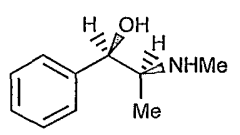

Ephedrine 3: respiratory ailments Ephedra spp.<smiles>COc1ccn([N+](=O)[O-])c(=O)c1C#N</smiles>

Ricinine 4 (castor oil): purgative Ricinus communis L.

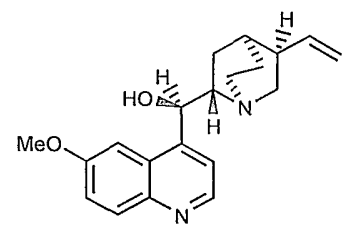

Quinine 5: Antimalarial Cinchona officinalis $\mathrm{L}$.

Fig. 1.1 Examples of natural products 
secondary metabolites are as much part of the genetic make-up of the organism as the primary metabolic pathways but they may only be activated at certain times to ensure the continued survival of the particular organism, for example in a hostile enviromment where many organisms are competing with each other, or at times of stress caused by nutritional limitations. The dividing line between primary and secondary metabolites is not a distinct one, with some primary metabolites having specialised uses more associated with the secondary metabolites. In the biosynthesis of secondary metabolites, the starting materials (or building blocks) are comprised of a small number of primary metabolites-perhaps suggesting an evolutionary process giving rise to the appearance of secondary metabolites. ${ }^{2}$ The secondary metabolites can thus be divided into three main classes according to the primary metabolites used in their biosynthesis; amino acids (including shikimate-derived metabolites) — which are precursors to alkaloids and penicillins; mevalonate-precursor to terpenes and steroids; and acetate-precursor to polyketides and fatty acids.

Many secondary metabolites are molecules that are both structurally and pharmacologically interesting. Within the sub-group of polyketides alone there is great diversity in the range of chemical structures and biological activity (Fig.1.2). These include antibiotics (e.g. erythromycin A 6 and tetracycline 7), antifungal agents (griseofulvin 8 and amphotericin B 9), anti-cancer agents (dynemycin 10) and immunosuppressives (rapamycin 11). 


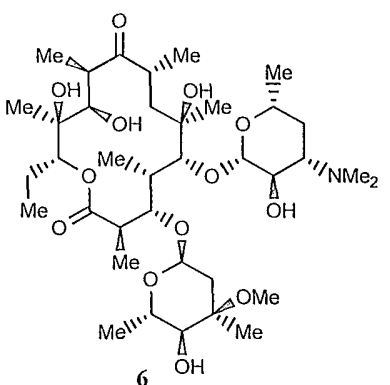

(Saccharapolyspora erythraea)

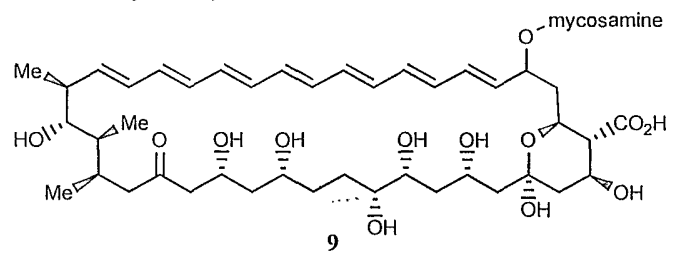

(Streptomyces nodosus)

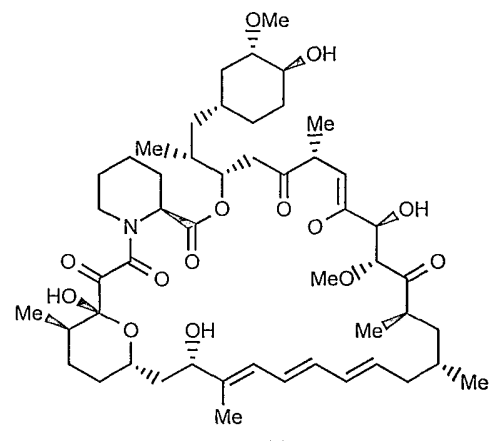

11

(Streptomyces hygroscopicus)

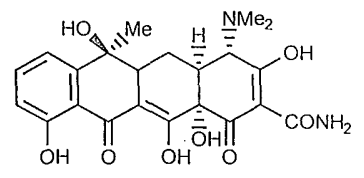

7

(Streptomyces

viridifaciens)

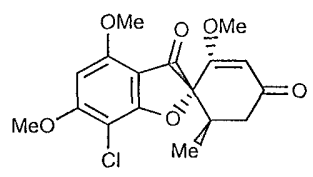

8

(Penicillium

griseofulvin)

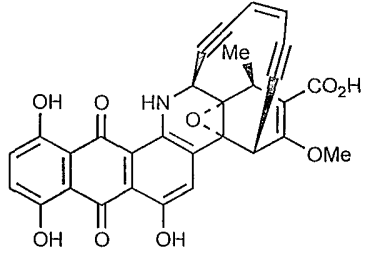

10

(Micromonospora

chersina)

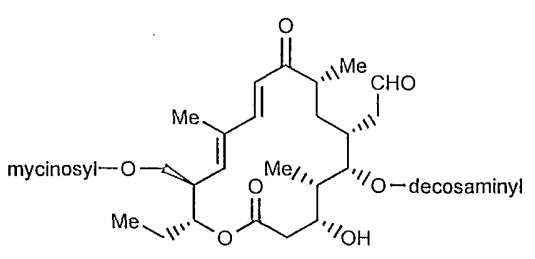

12

Fig. 1.2 Examples of polyketides 


\subsection{Evollution of polyketides}

\subsubsection{History}

It was in 1907 that the term polyketide was first introduced by Collie $^{3}$ when he recognised a number of aromatic plant metabolites were made up of $\left(\mathrm{CH}_{2} \mathrm{CO}\right)_{n}$ units. He provided some experimental evidence in support of his hypothesis ${ }^{4}$ but it was not until some years later that his ideas were investigated further. Work carried out by Robinson, ${ }^{5}$ and independently by Birch, ${ }^{6}$ who proposed the 'acetate hypothesis', stimulated by developments in fatty acid biosynthesis, discovered acetyl coenzyme A (acetyl $\mathrm{CoA}$ ) to act as the 'active acetate' in polyketide construction. ${ }^{7}$ By analogy with fatty acid biosynthesis, ${ }^{8}$ it was suggested that polyketides were formed by a head-to-tail condensation of acetate units. This basic acetate hypothesis still holds firm today, if in an extended form. Most of the polyketides discovered during early studies were of the 'aromatic' type, such as tetracenomycin, with chain extension solely by assembly of $\mathrm{C}_{2}$ acetate units. During later studies, however, it was realised that many polyketides are also constructed of larger sub-units and the acetate hypothesis has been extended to include larger chain starter, e.g. propionyl CoA in tylosin $12^{9}$ (Fig. 1.2) and chain extension units, e.g. methylmalonyl CoA in erythromycin A $6 .^{10}$ 
The initial stimulus into their research was provided by the significant developments in the understanding of fatty acid biosynthesis. The similarities between the structures of fatty acids and polyketides are evident, which appear to arise from the basic analogies in their biosynthetic processes. The basic origin of polyketides involves chain assembly of acetyl units by Claisen-like condensations in a manner that is reminiscent of fatty acid biosynthesis. The polyketides could be considered to be more complex fatty acids, which might suggest an evolutionary process between the two classes of metabolite." This complexity arises from a mixture of different chain starter and extension units (e.g. propionate and butyrate, as well as acetate) and a variety of levels of reduction at the $\beta$-carbonyl position. The absolute configuration of the resulting chiral centres along the carbon chain can be retained during subsequent extensions, which is another factor contributing to the complexity of the polyketides.

To begin to understand the assembly process of the polyketide metabolites, it would therefore be convenient to briefly consider the biosynthesis of the structurally simpler fatty acid metabolites.

\subsubsection{Fatty acid biosynthesis}

Fatty acids are present in almost all living cells where, among their various roles, they play an important part in the structural assembly of cell membranes as diglycerides. The almost ubiquitous distribution of fatty acids means they are 
classed as primary metabolites, and it is only the less common fatty acids that may be considered as secondary metabolites.

Fatty acids are biosynthesised from acetyl CoA and malonyl CoA by a series of condensations and reductions. A general scheme for fatty acid biosynthesis is shown in Fig. 1.3. The initial step in the sequence involves the carboxylation of acetyl CoA to give malonyl $\mathrm{CoA}$, which is then transesterified onto the fatty acid synthase (FAS) where it is covalently bound by an acyl carrier protein. The FAS is the group of enzymes responsible for the processes that occur during chain extension, in which the growing acyl chain is extended by the decarboxylative Claisen condensation of each subsequent malonyl sub-unit. The resultant $\beta$-keto acyl chain is then reduced (to $R-$ $\mathrm{OH}$ ), dehydrated (to produce an $E$ double bond) and further reduced to a methylene group, and the cycle is then repeated until the chain is of required length and is then released by the enzyme complex. It has been established that there are two types of fatty acid synthases based upon their structural organisation. ${ }^{12}$ Type I synthases occur in all animals, occasionally yeasts (all fungi) and some higher bacteria (e.g. some Mycobacterium spp.). Due to the fact that their individual catalytic sites are situated on a common polypeptide chain they cannot be separated from one another, and so they are described as multifunctional proteins. In contrast, type II synthases, found in plants and most bacteria, possess discrete enzymes that normally have a single activity and can be isolated separately. Fatty acid synthases have been investigated widely ${ }^{13,14}$ and one method to provide evidence for the enzymes involved has been to use fatty acid inhibitors. It has been shown that fatty acid biosynthesis 


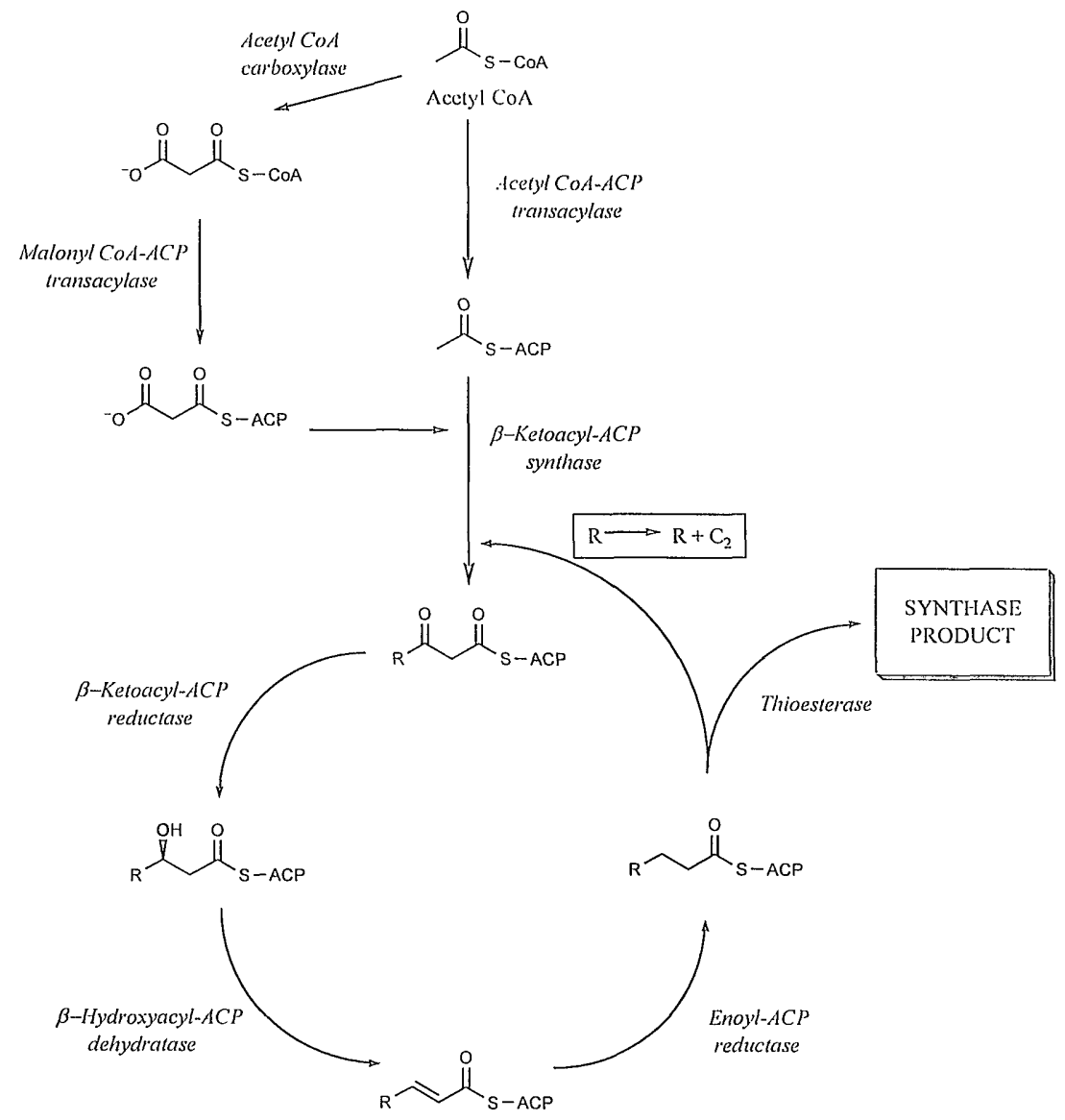

Fig. 1.3 Fatty acid biosynthesis

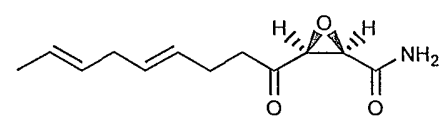

Cerulenin 13 
is inhibited by the antibiotic cerulenin 13 , which is isolated from Cephalosporium caerulens, which acts by blocking the condensing enzyme $\beta$-ketoacyl synthase. ${ }^{15}$ Cerulenin 13 has also been found to inhibit many polyketide synthases ${ }^{16-18}$ suggesting that the condensing enzyme in polyketide biosynthesis is similar to the analogous component in fatty acid biosynthesis.

\section{$\mathbb{1} .3$ Polyketide biosymthesis}

The biosynthesis of polyketides is thought to occur by a process similar to fatty acid biosynthesis, by a series of condensations and reductions, but further complexity is introduced by the involvement of propionate, butanoate and other starter and extension units as well as acetate. All polyketides can be imagined to derive from a basic carbon skeleton that is reminiscent of fatty acids but with variable levels of oxidation at each alternate carbon. The majority of polyketides are produced by the Gram positive bacteria, the actinomycetes, although many occur in other bacteria, with a number having also been found in fungi, marine, plant and other organisms. The actinomycetes produce polyketides that are from two basic origins and can be classified as either aromatic, such as tetracycline 7, or non-aromatic, such as erythromycin A 6. In all cases, the assembly of the polyketide chain is under the control of the enzyme complex that constitutes the polyketide synthase (PKS). As in fatty acid biosynthesis, once the acyl chain has reached its required length it is released by the PKS, after which it may undergo further modifications and 


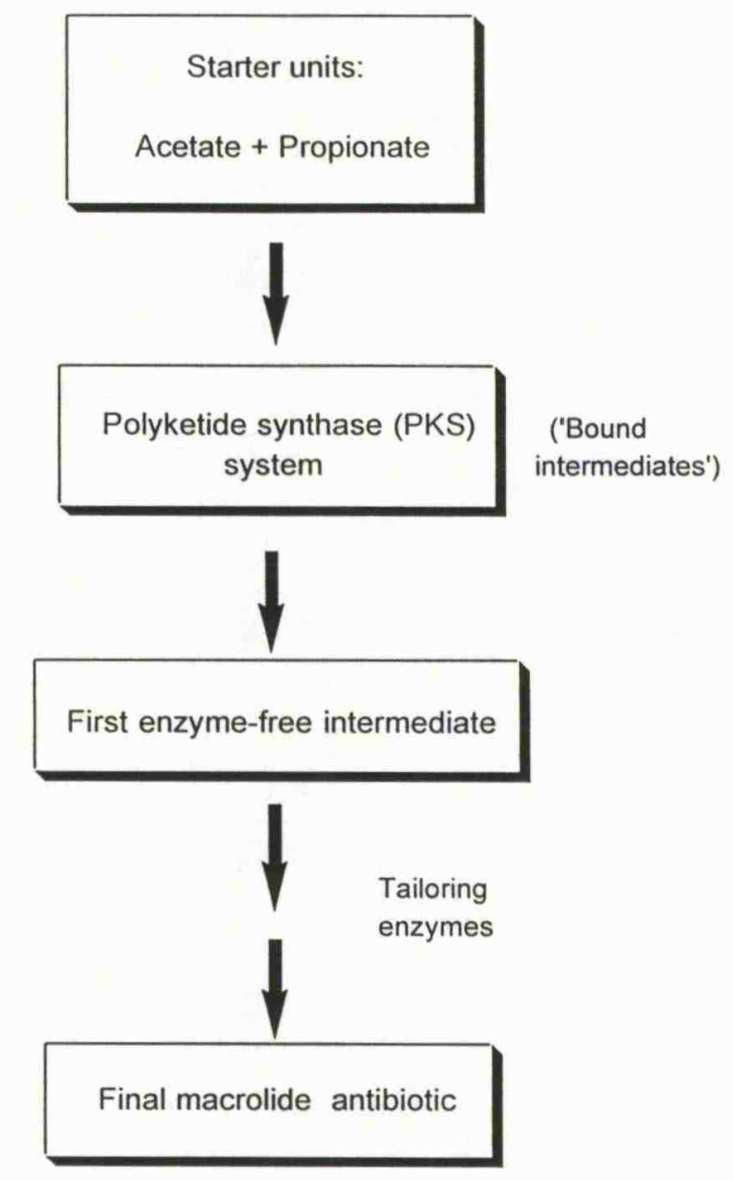

Fig. 1.4 General scheme of polyketide biosynthesis

cyclisations in order to produce the ultimate metabolite (Fig. 1.4). The mode of assembly of the acyl chain by the PKS varies between the aromatic and the nonaromatic polyketides, the latter of which will be dealt with in more detail here, with only a brief discussion of the biosynthesis of aromatic polyketides. 


\subsubsection{Aromatic Polyketides}

The structural skeleton of the aromatic polyketides are generally made up exclusively of acetate sub-units. Once released from the PKS the polyketide chain undergoes cyclisation which may take several different forms, as illustrated for the tetraketide in Fig. 1.5. Subsequent oxidations or decarboxylations may then take place on the 'free' PKS products. The metabolic products can be formed from the unmodified polyketide chain, such as in orsellinic acid 13 (Fig. 1.6), or undergo modifications before (reductions or dehydrations e.g. 6-MSA 14) or after aromatisation (phenolic coupling e.g. usnic acid 15).

When the compound is released from the PKS and it undergoes cyclisation, some aromatic polyketides can then undergo further modifications, for example decarboxylations, $O$-methylation or oxidations. In Fig. 1.7 the biosynthesis of the fungal anthraquinone emodin 16 can be seen to arise from the intramolecular condensation of an octaketide, to give the cyclised intermediate shown, which if oxidised and decarboxylated produces emodin.

\subsubsection{Non-aromatic polyketides}

The assembly of the polyketide skeleton of the non-aromatic structures is again under the control of the PKS but generally involves a combination of acetate, propionate and butyrate sub-units with the occasional more unusual chain extension units, such 


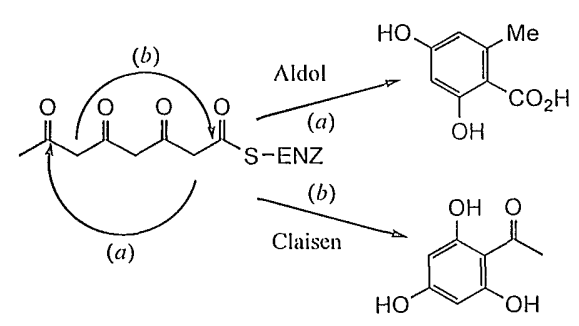

Fig. 1.5 Cyclisations of aromatic polyketides

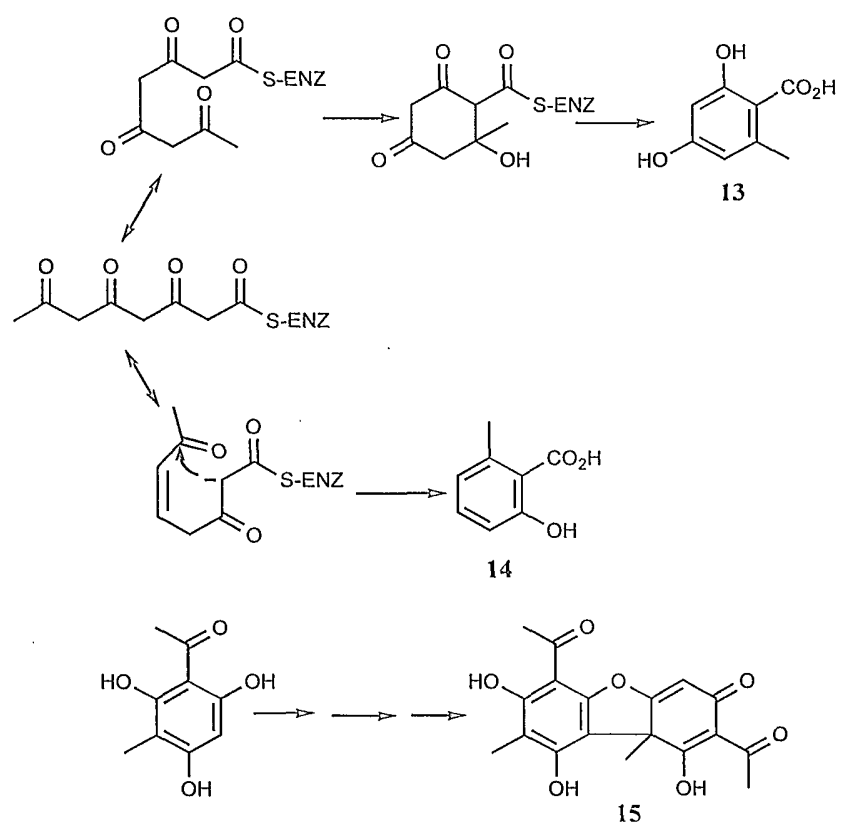

Fig. 1.6 Formation of aromatic polyketides

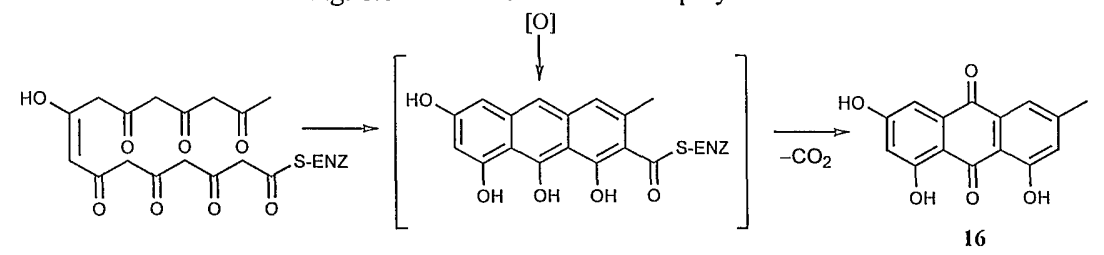

Fig. 1.7 Condensation to form emodin 
as octanoate in fungichromin. ${ }^{19}$ Once the acyl chain has reached the required length and is released by the PKS to give the first enzyme-free product, this may then undergo further modifications and cyclisation to furnish the final metabolite. There are three main classes of non-aromatic polyketide produced by the actinomycetes, which arise due to the various modes of cyclisation. These are the polyethers, macrolides and polyenes.

The structurally modified polyketide chains can, in the first instance, undergo a concerted cyclisation via polyepoxides to produce polyethers by the 'cascade mechanism'. The theory was used to explain the biosynthesis of lasalocid-A 17 , which was among the first of this class of antibiotics to be isolated. ${ }^{20}$ Westley et al. proposed a mechanism as in Fig. 1.8 which involved the formation of the diepoxide 18 that could then undergo the cascade cyclisation, which has since been proved with labelling studies. ${ }^{21}$ Further evidence was provided for this hypothesis with ${ }^{13} \mathrm{C}-{ }^{18} \mathrm{O}$ feeding experiments by Cane in the formation of monensin A $19^{22,23}$ (see p.17), which has since led to the generalised theory of polyether formation. ${ }^{24}$ The second mode of cyclisation involves lactonisation, which results in the formation of macrocyclic rings, that can be divided into two classes-those that possess a polyene chromophore within the macrocyclic ring, which are termed polyenes (e.g. nystatin 20), and those that do not, which are described as macrolides (e.g. tylosin 21). Both forms may be further modified after macrolactonisation by 'tailoring' enzymes to produce the final metabolite. 


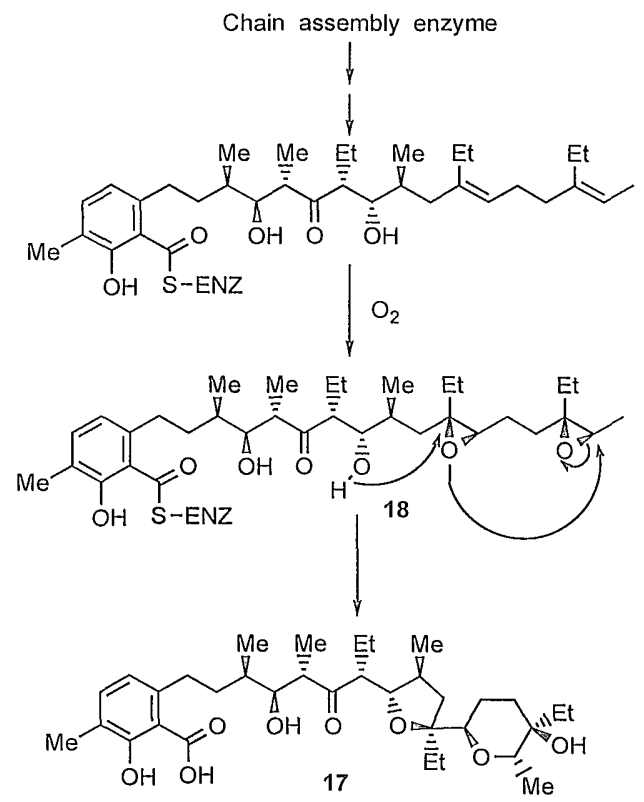

Fig. 1.8 Cascade mechanism for the biosynthesis of lasalocid A

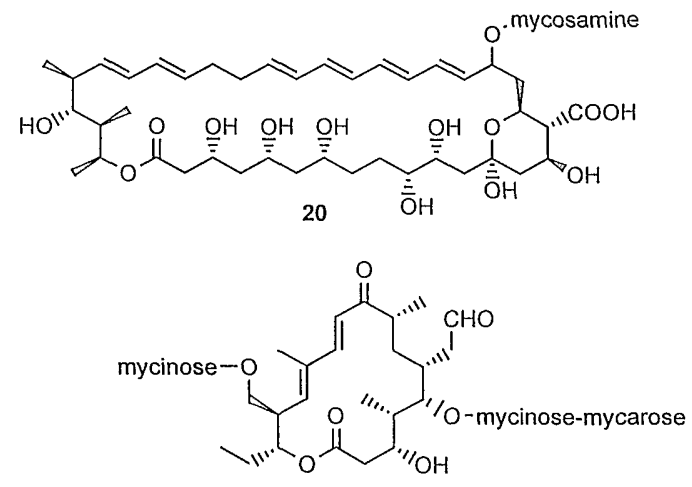

21 
Some of the first investigations into the biosynthesis of the non-aromatic polyketides involved the macrolide erythromycin B 22 produced by Saccharopolyspora erythraea. Early studies into the biosynthesis of this metabolite involved the use of mutants. Intermediates in the latter stages of the biosynthetic pathway can be studied by the aid of inhibitors and blocked mutants. ${ }^{25,26}$ Mutants can be obtained (by genetic or chemical disruption) that are unable to produce a specific enzyme in the biosynthetic pathway, which results in the accumulation of the intermediate prior to the step where the enzyme is inactive, which if isolated in sufficient quantities may provide some information about the late stages of the biosynthesis. In the case of erythromycin, several late intermediates in the biosynthetic pathway were isolated in this way, with the simplest of these being the aglycone of erythromycin, 6deoxyerythronolide B 23, which was postulated as the first enzyme-free intermediate. $^{27}$

The basic backbone of erythromycin is made up of seven propionate sub-units and it was from feeding experiments to cell cultures of $S$. erythraea with $\left[1-{ }^{13} \mathrm{C}\right]$ and $\left[2-{ }^{13} \mathrm{C}\right]$ propionate that the carbon skeleton was elucidated to be constructed of one unit of propionyl CoA and six units of methylmalonyl CoA. ${ }^{10}$ Thus, the component parts of the structure of erythromycin had been identified, but the process by which these were assembled by the PKS to produce the first enzyme-free intermediate, 6deoxyerythronolide B 23, was still not clear. 

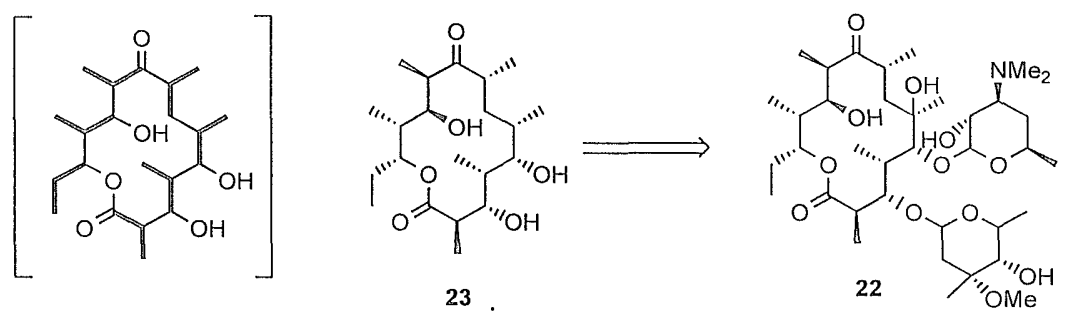

\subsubsection{Processive vs. non-processive hypothesis}

The process of polyketide chain assembly in non-aromatic polyketides must now be considered in more detail. Singly and doubly labelled ${ }^{13} \mathrm{C}$-acetate, propionate or butyrate units have been used extensively to determine the origin of the carbon skeleton. ${ }^{28}$ Such feeding experiments have demonstrated that the carbon backbones are generally made up of a combination of acetate, propionate and butyrate sub-units (e.g. monensin A 19 and rifamycin B 24, Fig. 1.9), with the occasional more unusual chain extension units, such as octanoate in fungichromin $46 .{ }^{29}$

From this selection of non-aromatic polyketides, the broad range of types of metabolite can be seen and already the complexity of the structures is such that it would be difficult to assign certain ones, i.e. rifamycin, to any one of the categories of polyketide already mentioned. Thus, a combination of the different sub-units of the structural skeleton, the various fates of the alternate keto groups and the length of the polyketide chain, along with the events that occur after chain assembly, account 


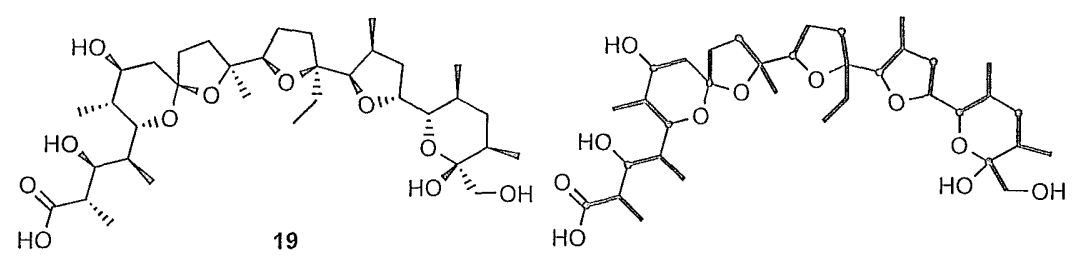

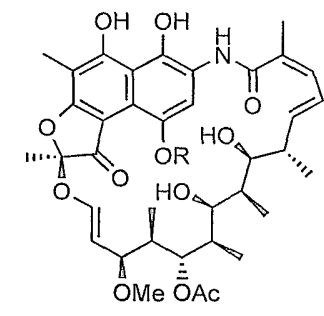

24

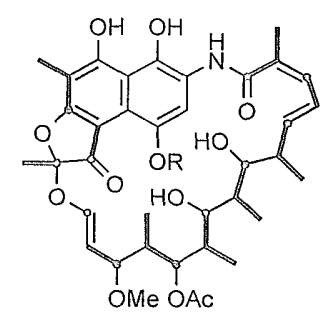

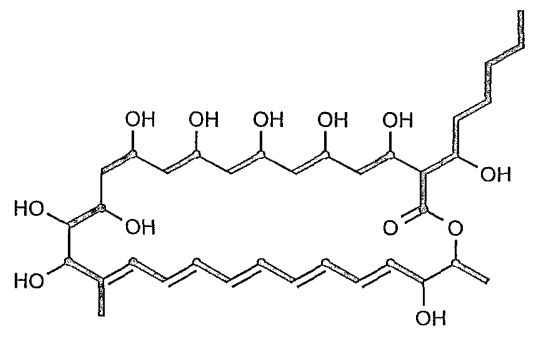

46

Fig. 1.9 Illustration of the acetate, propionate and butyrate skeleton

in some non-aromatic polyketides. 
for the great diversity among these compounds. The selections that the PKS makes in constructing a certain metabolite result in only one out of the numerous theoretically possible carbon chains, and thus the key to understanding the biosynthetic processes involved would be to investigate the programming, structure and function of the PKS itself.

It was considered that the detailed assembly process of the polyketide chain was likely to occur in one of two ways. The 'non-processive' hypothesis involves the formation of the carbon chain under the control of the PKS, with the processing of the observed functionality occurring after the chain assembly is complete or the 'processive' hypothesis in which the growing polyketide chain is modified prior to the addition of the next extension unit (Fig. 1.10). It is the processive pathway that is now considered the more favoured route and so a more detailed scheme of events was proposed (Fig. 1.11) that is reminiscent of the fatty acid biosynthetic cycle. In this hypothesis the pathway proceeds by the condensation of a starter unit (acetyl or propionyl etc.) with chain extension units (malonyl or methylmalonyl CoA). To produce the required oxidation state, after each decarboxylative Claisen condensation, the required functionality is established prior to the next condensation. The chirality of the propionate or butyrate alkyl units introduced into the growing acyl chain is not generally lost during subsequent reactions, thus adding to the structural diversity of this class of metabolites. The cycle is then repeated until the modified acyl chain thioester is of required length and then the first enzyme-free 


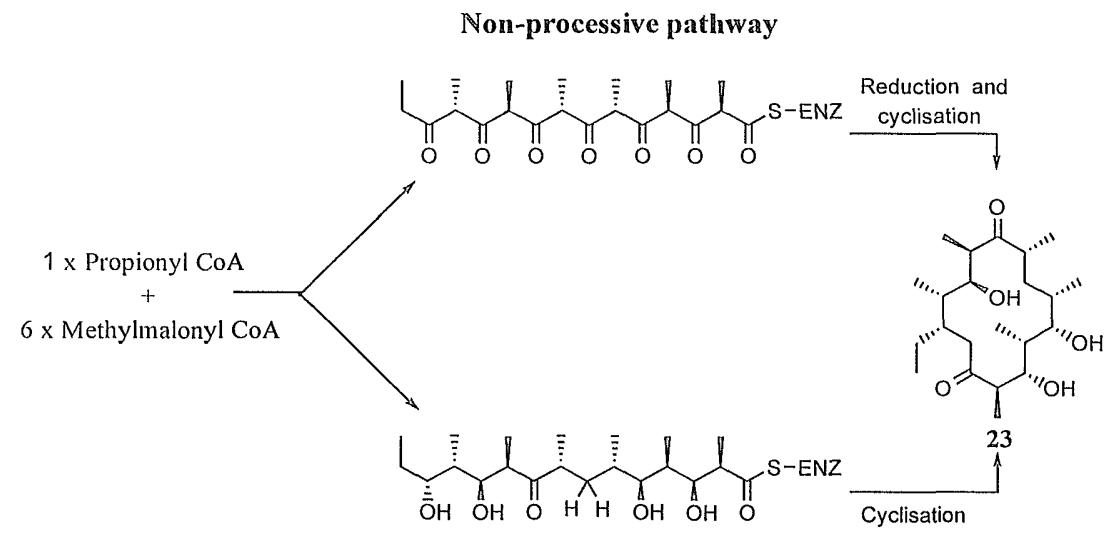

Processive pathway

Fig. 1.10 Two possible routes to the assembly of the polyketide chain in erythromycin biosynthesis

intermediate can be released in stable form. This then undergoes secondary modifications and cyclisations to give the desired polyketide.

In order to determine whether this is indeed the biosynthetic process that the PKS undertakes rather than the non-processive pathway, evidence must be provided to support the hypothesis. This evidence may arise through a variety of experimental procedures including the incorporation of proposed intermediates into the final metabolite, the isolation of PKS partly-assembled intermediates and ultimately the isolation and understanding of the PKS itself. 


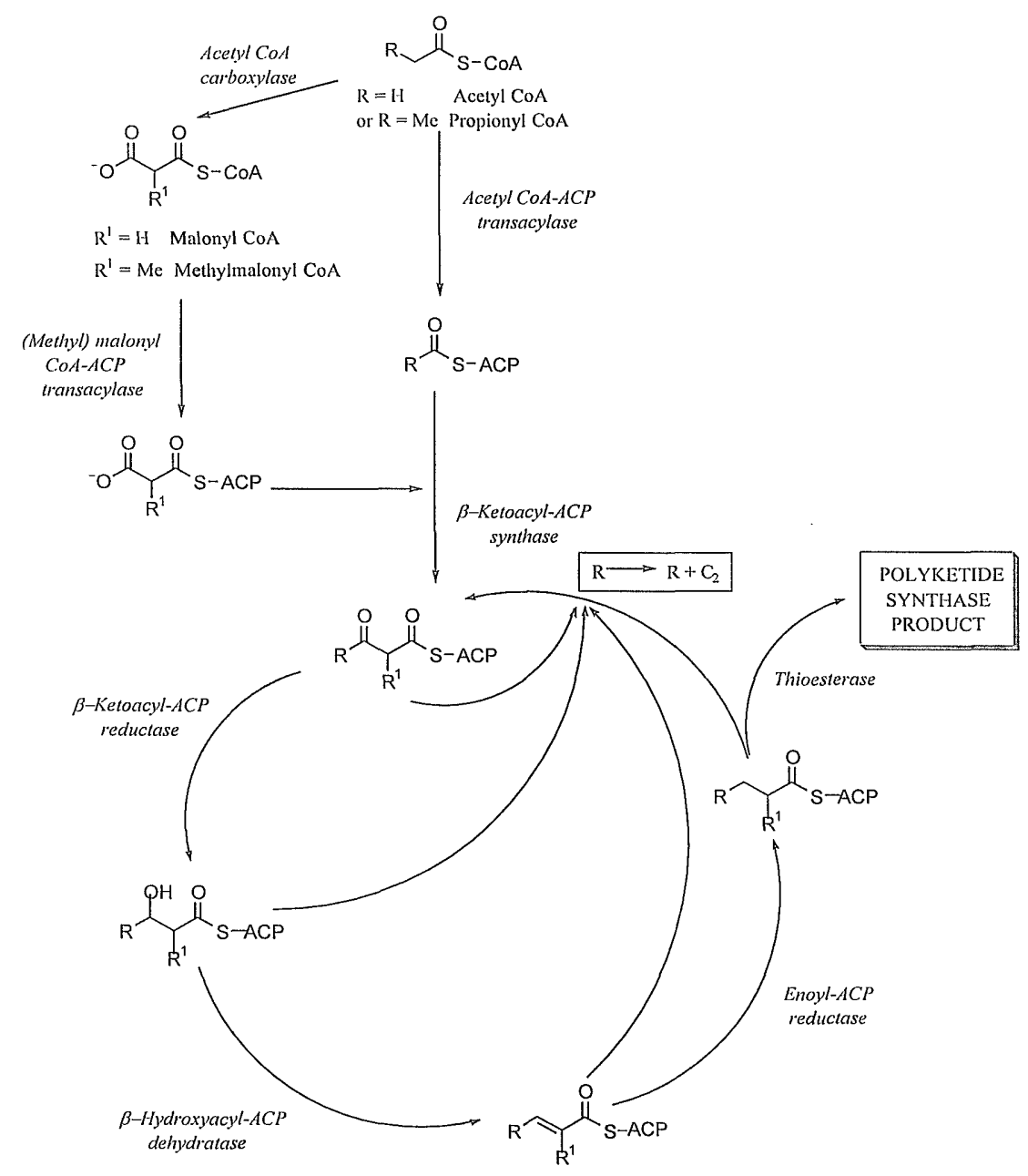

Fig. 1.11 Polyketide biosynthesis; processive hypothesis 


\subsubsection{Evidence for the processive hypothesis}

By far the most extensive experiments have involved the intact incorporation of precursors into a variety of macrolide antibiotics. The first key evidence for the processive hypothesis was provided by intact incorporation of labelled di- and triketide labelled elongation intermediates into tylosin ${ }^{30}$ and erythromycin. ${ }^{31}$ The diketides that were fed in these investigations involved the use of singly and doubly labelled acetate units.

In a study carried out by Hutchinson et al, a variety of labelled intermediates were incorporated intact into tylactone $25^{30}$ (the parent aglycone of the 16 membered macrolide, tylosin) (Fig.1.12). This investigation involved the incorporation of ${ }^{13} \mathrm{C}$ singly labelled precursors at both the di- and tri-ketide stages and the incorporation detected by NMR.

However, it has been the use of doubly labelled precursors that have provided much of the evidence for the processive pathway, e.g. diketide precursors containing two carbon-13 atoms sited adjacent to each other but situated in different 'acyl units', if incorporated intact into the metabolite, each produce a doublet in the ${ }^{13} \mathrm{C}$ NMR spectrum which is superimposed over the natural abundance signal for that carbon atom. The persistence of coupled signals after incorporation suggests that the bond 


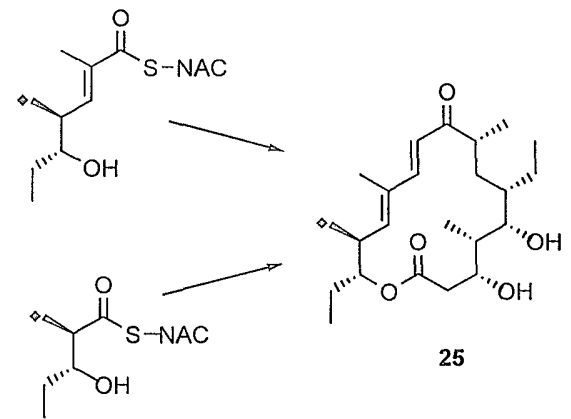

Fig. 1.12 Incorporation of di-and tri-ketides into tylactone $25 ; \diamond^{13} \mathrm{C}$ label

between the coupled carbons has remained intact during biosynthesis, thus providing evidence that the incorporated precursor is indeed a true intermediate.

Investigations into the biosynthesis of erythromycin involved the use of doubly labelled intermediates, in which the postulated processive diketide precursor 26 was synthesised and administered to cultures of S. erythaea. ${ }^{31}$ The intact incorporation of the $N$-acetylcysteamine derivative of $\left[2,3-{ }^{13} \mathrm{C}_{2}\right]-3$-hydroxy-2-methylpentanoic acid 26 into erythromycin B 22 (Fig. 1.13) was detected by the presence of enhanced and coupled doublets in the NMR spectrum. Initially however in this study, and that of Hutchinson et al., the free acid was fed but this experiment resulted in extremely low levels of intact incorporation, which suggested the precursor had been degraded by fatty acid oxidases to give a mixture of $\left[1-{ }^{13} \mathrm{C}\right]$ and $\left[2-{ }^{13} \mathrm{C}\right]$ propionate, which were detected by NMR to have been randomly incorporated into the macrolide. The successful incorporation of the NAC thioester derivative 27 is considered to be due to 
the fact it can undergo transesterification onto the enzyme thiol group more easily than the oxygen of the free acid because of the structural homology to the thiol end of coenzyme A $(\mathrm{CoA}) 29$ and the acyl carrier protein phosphopantetheine chain 28

(Fig. 1.14).

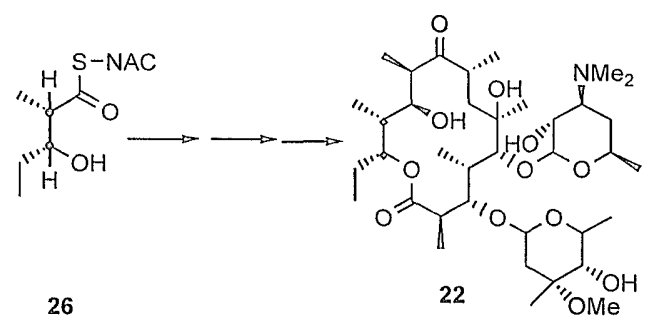

Fig. 1.13 Incorporation of the labelled diketide 26 into erythromycin B $22 ;{ }^{\circ}{ }^{13} \mathrm{C}$ label

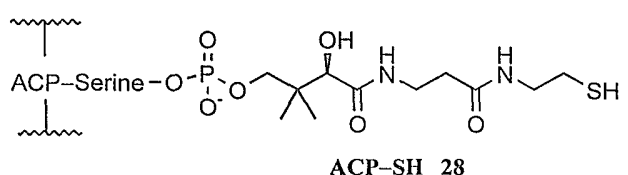

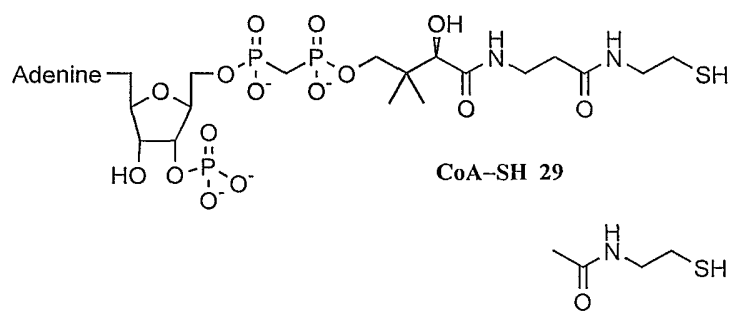

NAC-SH 27

Fig. 1.14 Comparison of the thioester $N$-acetyl cysteamine (NAC) 27 with the thiol part of the terminus of ACP 28 and $\mathrm{COA} 29$ 
These techniques have been utilised in the study of the biosynthesis of a number of polyketides, with the Cane group going on to incorporate ${ }^{13} \mathrm{C}-{ }^{13} \mathrm{C}$ labelled di-, triand tetra-ketide precursors into the alicyclic polyketide nargenicin 30 (Fig. 1.15). ${ }^{32}$

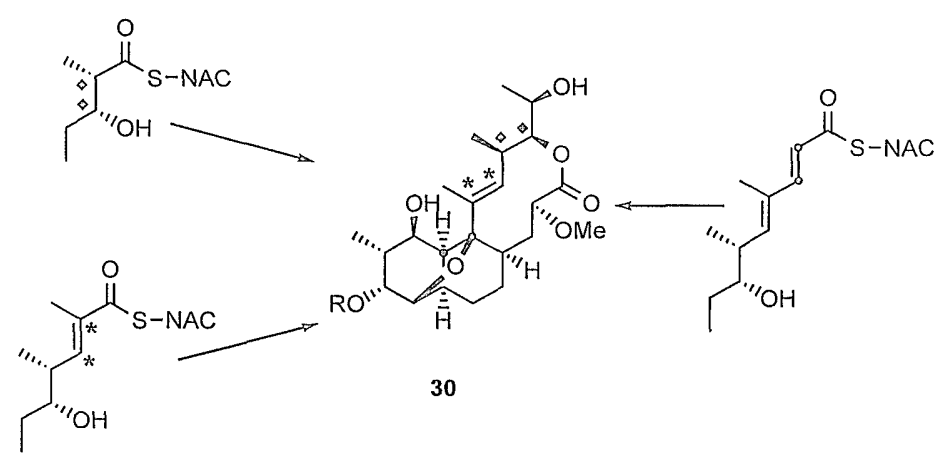

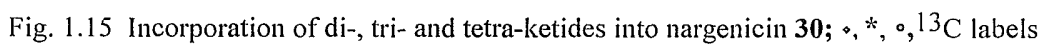

Due to the fact that experiments such as these can be hampered by the rapid degradation of the labelled precursor to acetate (or propionate) by $\beta$-oxidation, it has been shown that the use of $\beta$-oxidation inhibitors such as 4-pentynoic acid 31 and 3(tetradecylthio)propanoic acid 32 increases the possibility of intact incorporation by suppressing the breakdown of the precursor. ${ }^{32,33,34,35}$ Other solutions to the problems such as the solubility and uptake of the precursor must be optimised and the use of 2,6-O-dimethyl- $\beta$-cyclodextrin 33 in studies of the biosynthesis of monensin A 19, has shown to stimulate the production of monensin and possibly also the uptake of the precursor into the metabolite. ${ }^{36}$ 


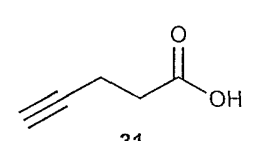

31
$\mathrm{Me}\left(\mathrm{CH}_{2}\right)_{13}-\mathrm{S} \sim$

32

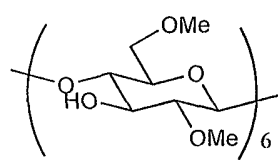

33

Various combinations of isotopic labels may be used in biosynthetic precursors. Intermediates having ${ }^{13} \mathrm{C}$ as the reporter nucleus have been used to establish the integrity of ${ }^{13} \mathrm{C}-{ }^{2} \mathrm{H},{ }^{13} \mathrm{C}-{ }^{12} \mathrm{C}-{ }^{2} \mathrm{H}$, and ${ }^{13} \mathrm{C}-{ }^{18} \mathrm{O}$ bonds after the precursors have been incorporated into the metabolite. If a carbon has one deuterium or ${ }^{18} \mathrm{O}$ attached there is an observable shift in the ${ }^{13} \mathrm{C}$ NMR spectrum, which is described as the $\alpha$-shift. If the heavy isotopes are one bond removed from the ${ }^{13} \mathrm{C}$ atom, there is what is described as a $\beta$-shift. ${ }^{37,38}$ There has been successful incorporation of advanced intermediates containing deuterium labels into aspyrone $34^{39,40,41,42}$ and tetronasin $35^{43,44}$ and a variety of studies involving doubly labelled ${ }^{13} \mathrm{C}$ intermediates in combination with ${ }^{18} \mathrm{O}$ labels incorporated into dehydrocurvularin 36 (Fig. 1.16). ${ }^{33,34}$

In the biosynthesis of methymycin 37 , the ${ }^{13} \mathrm{C}-{ }^{13} \mathrm{C}$ labelled compound 38 was administered to cultures of Nocardia argentinensis and the level of incorporation was determined by the set of enhanced and coupled doublets corresponding to the ${ }^{13} \mathrm{C}$ 

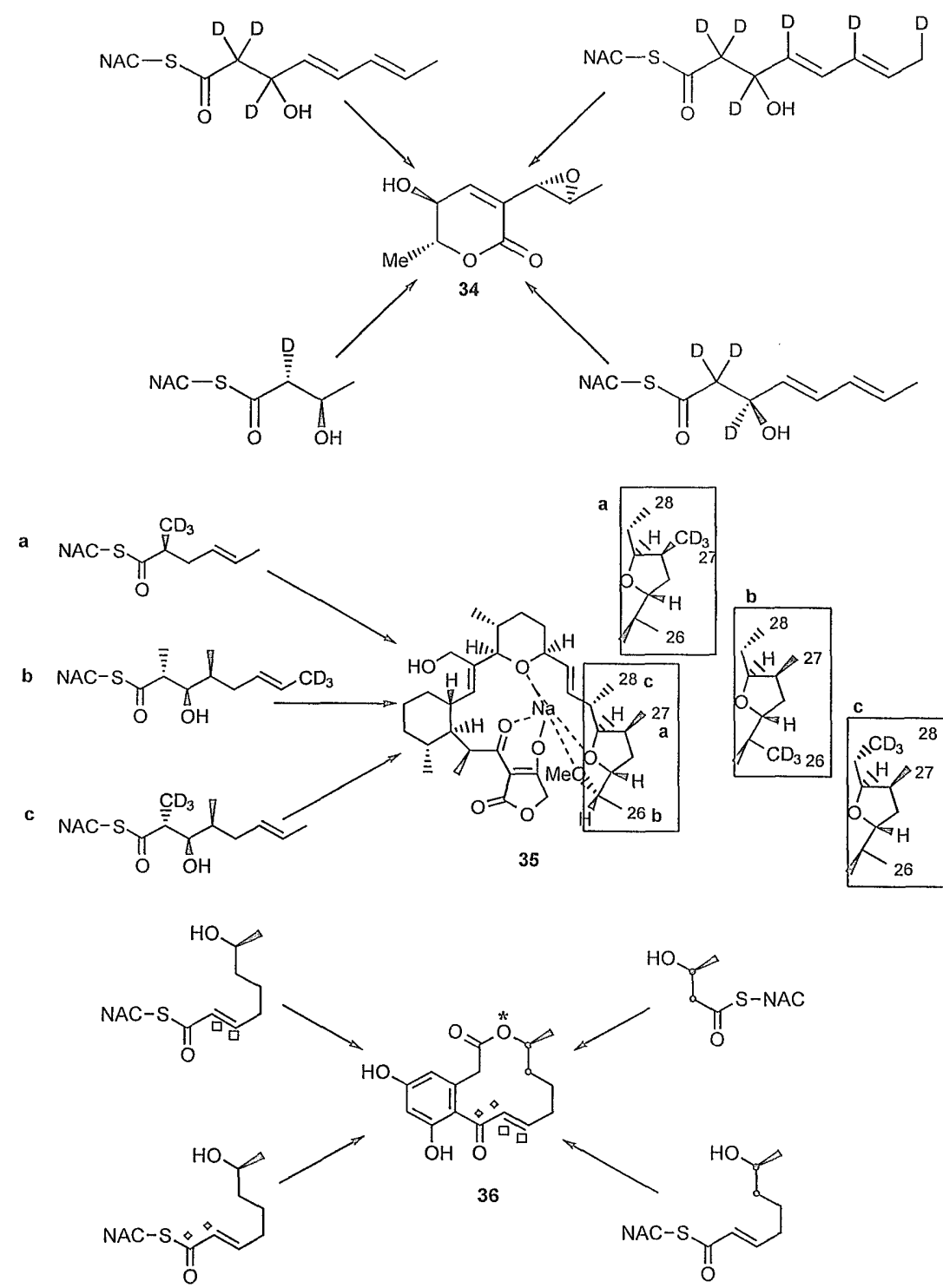

Fig. 1.16 Incorporation of labelled intermediates into aspyrone 34, tetronasin 35 and dehydrocurvularin $36 ;{ }^{*}$ acetate $18 \mathrm{O} ; \diamond,{ }^{13} \mathrm{C}$ labels 
labels (Fig.1.17). ${ }^{35}$ In order to rule out the possibility of re-oxidation of the diketide (of the 3-hydroxy functionality to the 3-keto) before incorporation (suggesting the non-processive hypothesis) the corresponding ${ }^{13} \mathrm{C}-{ }^{2} \mathrm{H}$ compound 39 was prepared and administered. The outcome of this experiment was the incorporation of the deuteriated thioester, displaying a ${ }^{13} \mathrm{C}-{ }^{2} \mathrm{H}$ triplet which collapsed to a singlet on ${ }^{2} \mathrm{H}$ decoupling.

The majority of studies to date have utilised both ${ }^{1} \mathrm{H}$ and ${ }^{13} \mathrm{C}$ NMR spectroscopy to detect intact incorporation of labelled precursors. Developments in mass spectrometry have meant that techniques such as fast atom bombardment (FAB) and electrospray (ESMS) can be employed, which are advantageous as only a very small sample size is required and the number of isotopic atoms per molecule can be determined. These techniques allow the molecular ion $\left(\mathrm{M}^{+}\right)$of large molecules to be<smiles>CC[C@H](O)[C@H](C)C(=O)SNC(C)(C)O</smiles>

38

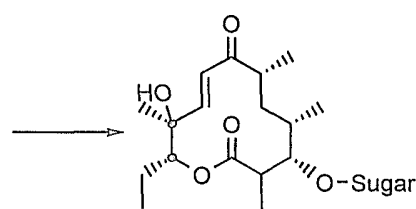

37<smiles>CCC(=O)[C@H](C)C(=O)SNC(=O)CC(C)C</smiles>

39

37

Fig. 1.17 Incorporation of labelled intermediates into methymycin 37 
detected and thus are often the mass spectrometric method of choice for proteins and biomolecules. Low levels of incorporation are difficult to detect but the use of a multiple labelling technique can eradicate this problem. For example, incorporation of a $\mathrm{CD}_{3}$ group is indicated by enhancement of the $\mathrm{M}+3$ peak. Recent investigations into the biosynthesis of avermectin CHB2 40 have utilised both NMR and electrospray mass spectrometric techniques to detect incorporation. Variously labelled precursors at the diketide stage were administered to cultures of Streptomyces avermitilis and intact incorporation was detected by the methods mentioned (Fig. 1.18). ${ }^{45}$

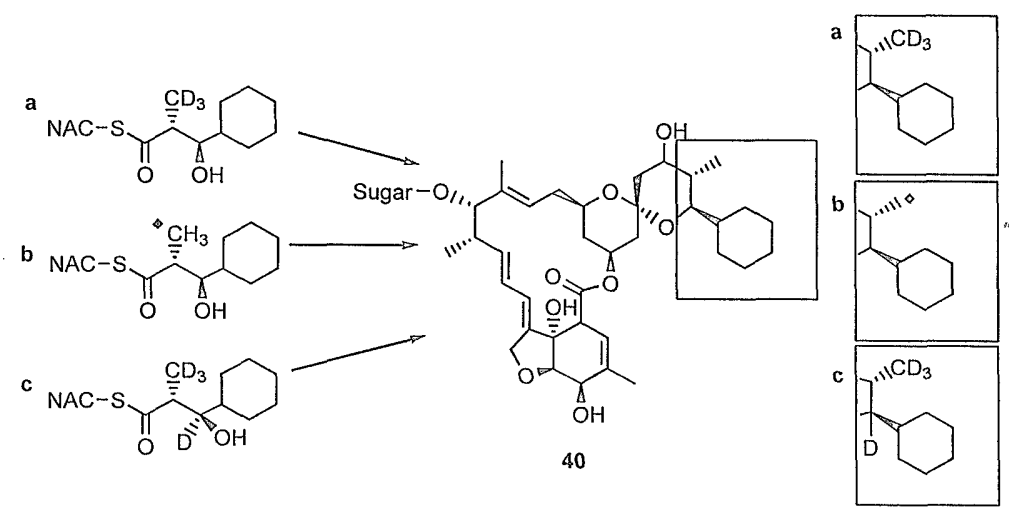

Fig. 1.18 Intact incorporation of precursors into avermectin CHB2 $40 ; \diamond 13 \mathrm{C}$ label 


\subsubsection{Further evidence: genetic analysis}

The area in which much research has been undertaken most recently has been the study of cell-free systems and purified enzymes which has been made possible by the location and over-expression of genes responsible for polyketide biosynthesis. The first polyketide synthase to be extracted and studied in detail was the 6methylsalicyclic acid (6-MSA) synthase from the fungus $P$. patulum. $^{46}$ 6-MSA is one of the simplest known polyketides and its PKS resembles that of a type I FAS, with all catalytic functions thought to be present on one polypeptide chain.

The study of the PKS from other organisms is much less well advanced and until the location of PKS genes in the mid 1980s, no other PKS enzyme could be isolated and purified. However, genetic analysis of a number of systems have now led to the isolation of a variety of biosynthetic genes. A great deal of work was carried out on the biosynthesis of the 'aromatic' polyketide actinorhodin 41 in S. coelicolor. ${ }^{47}$ The genes were found to encode for a series of discrete type II proteins, with each function catalysed by a separate protein involved in the biosynthesis i.e. a single ketoreductase (KR), acyltransferase (AC), acyl carrier protein (ACP) and thioesterase etc.

The nature of the PKSs of a variety of other aromatic polyketides have now been elucidated, ${ }^{48}$ e.g. for granaticin $42(\mathrm{gra})$, frenolicin 43 (fren) and medermycin 44 (med) (Fig. 1.19). The organisation and gene sequence for these metabolites appears 
to have close homology to that of actinorhodin $(a c t)$ and through the manipulation of the genes of the various PKSs, novel aromatic polyketides have been produced. The first hybrid polyketide was produced when the act gene responsible for the postassembly hydroxylation at the C-8 in actinorhodin was introduced into the DNA of the Streptomycetes that produces medermycin. The result of this experiment was the production of mederrhodin 45 , a compound which differs from medermycin only in the hydroxy group present at C-8. ${ }^{49}$ Since this early work, much further engineerings of aromatic PKSs have occurred (gene insertion, deletion etc.) with the consequent formation of a whole host of novel metabolites..$^{50-55}$

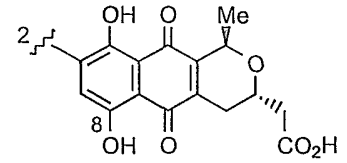

actinorhodin 41<smiles>C[N+](=O)[O-]</smiles>

frenolicin B 43

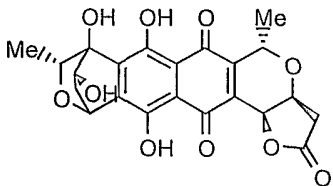

granaticin 42

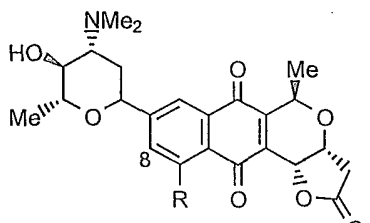

medermycin $44(\mathrm{R}=\mathrm{H})$ mederrhodin $45(\mathrm{R}=\mathrm{OH})$

Fig. 1.19 Aromatic polyketides for which the gene sequence has been determined 
However, there appear to be differences in the type of structure possible for PKSs, with the non-aromatic polyketides having PKSs that are not discrete. Studies on erythromycin in S. erythraea have shown the presence of three multifunctional proteins rather than a series of discrete enzymes. It would therefore appear that the PKS of erythromycin is analogous to a multifunctional type I rather than the dissociated type II FAS. ${ }^{27,48,56}$ It appears that in the PKS gene there are three large open reading frames (ORFs) each of which code for a single giant multifunctional protein, with each having sufficient catalytic sites for two chain extension cycles (Fig.1.20). It can be seen that the three genes appear to be organised into six modules or synthase units, each of which contain the active sites for a chain condensation step. Each module also contains some or all of the active sites for conversion of a keto group right through to a methylene group. The discovery of this extraordinary PKS, with its size and sheer complexity, is of great interest and breaks new ground. The information obtained from sequencing genes gives a fascinating insight into the primary organisation of the PKS proteins and some indication of how they function.

Recent genetic engineering studies into the PKS of erythromycin have resulted in the production of novel metabolites. As there is a separate catalytic activity for each reaction required for the processive assembly of erythromycin $B$, the disruption of any one of these activities may lead to the production of new erythromycins. For example, the disruption of the ketoreductase gene in module 5 of the PKS of erythromycin resulted in a macrolide that had a keto functionality at C-5 instead of 
the usual hydroxy group. ${ }^{57} \mathrm{~A}$ variety of other experiments involving the expression of certain parts of the PKS e.g. the ORF1 gene containing only modules 1 and 2 [Fig. 1.20 , path $(h)]$, have been carried out with the successful production of macrolides having various six-membered lactones. ${ }^{58,59}$

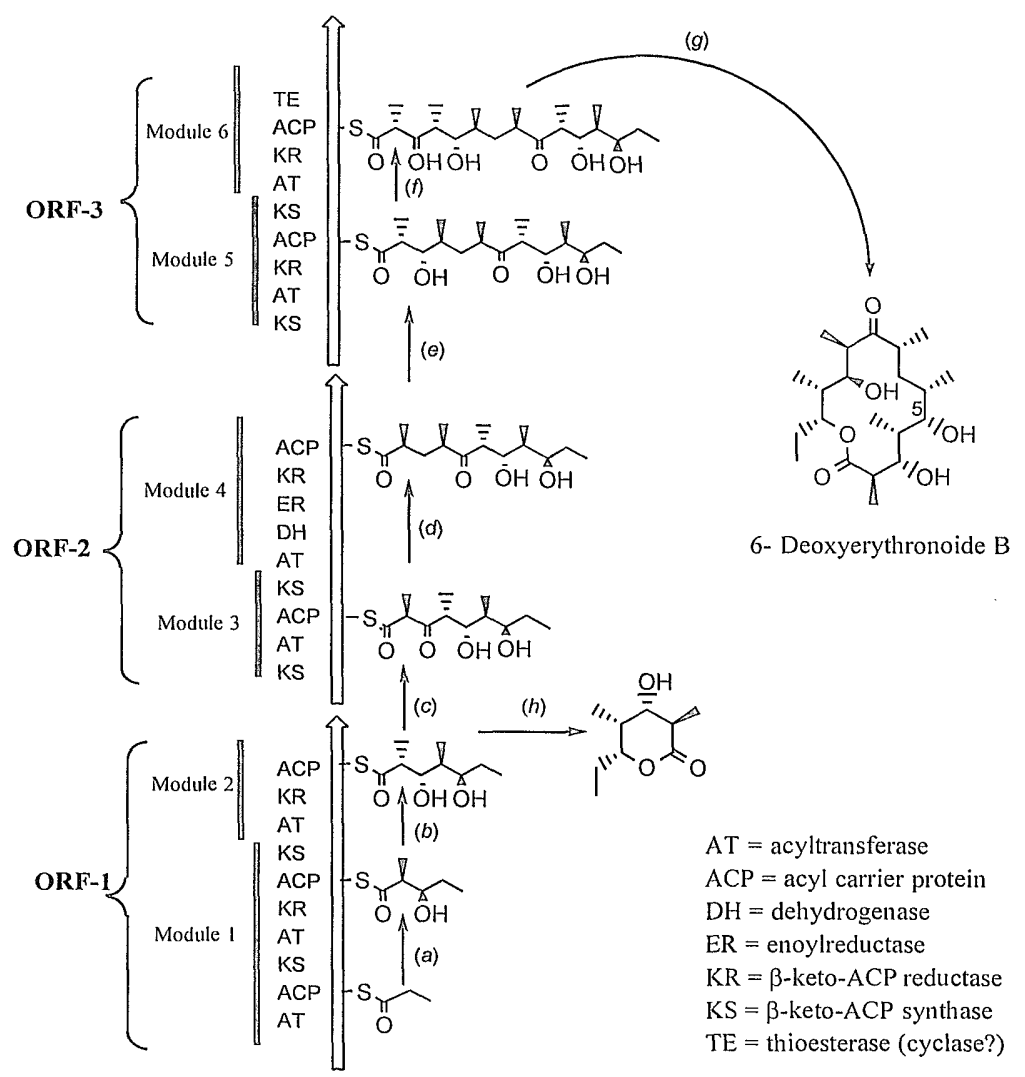

Figure 1.20 The genes of the erythromycin polyketide synthase 
With the various methods of investigation used to gain an understanding of the assembly of polyketides and the enzyme systems involved, an insight is being gained into the structure and function of the PKS. Thus, as our knowledge of the polyketide synthase increases, so does the enormous potential for the production of novel compounds.

\subsection{Amphotericin $\mathbb{B}$}

\subsubsection{Background}

Amphotericin B 9 is a member of the polyene class of polyketide metabolites. Other examples of this family can be seen in Fig. 1.21, where the characteristic hydrophobic chain of the conjugated double bonds can be seen on one side of the structure, with hydrophilic variety of hydroxy moieties on the other. ${ }^{60,61}$ In recent years there has been little research into the understanding of the biosynthesis of polyenes, with the exception of fungichromin $46,{ }^{29,62}$ possibly because of the inherent problems encountered when working with these metabolites and, in particular, with amphotericin B, its insolubility and intractability etc.

Amphotericin B 9 is produced by the soil bacterium Streptomyces nodosus (along with its co-metabolite amphotericin A) and was first isolated from the region of the Orinoco River in Venezuala in $1955 .{ }^{64-66}$ It is an antibiotic for many topical 

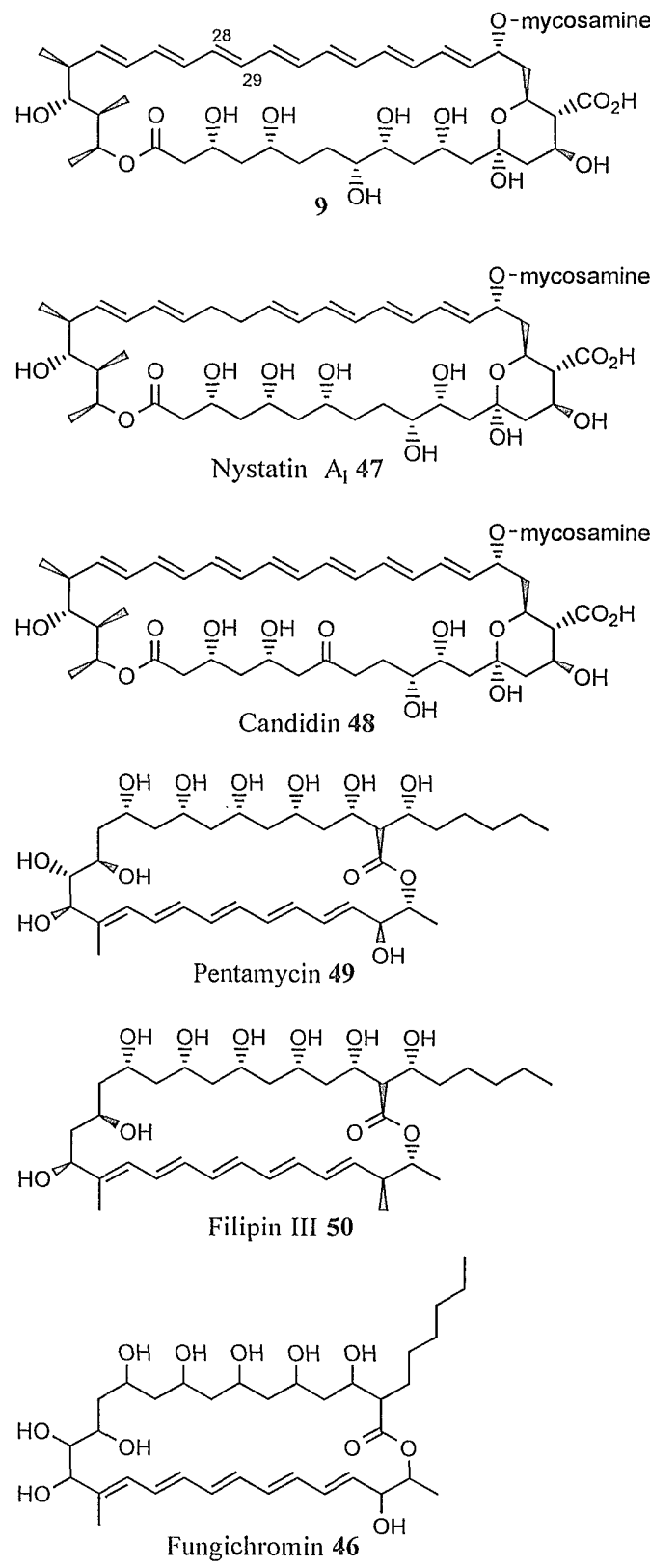

Fig. 1.21 Examples of polyene metabolites 
infections and has been used for over thirty years as a lifesaver against systemic fungal infections, ${ }^{67}$ even though it is toxic to humans.

\subsubsection{Mode of action}

The proposed mode of action $^{68}$ of amphotericin B makes use of both the hydrophilic and hydrophobic properties of the structure. It is believed to involve the complexation of eight steroid molecules, such as ergosterol in fungal cells, with eight amphotericin B molecules in the cell membrane to form a cylindrical/tubular supramolecular complex which acts as a half-pore within the fungal cell membrane. ${ }^{69}$ The dynamic nature of the cell membrane bilayer enables alignment of these half pores thus forming an ionic channel which causes the loss of potassium and sodium ions from within the cell, leading to cell death due to loss of osmotic control. The toxicity to mammalian cells arises from the ability of amphotericin B to also bind with mammalian sterols, such as cholesterol, producing side effects including tinnitus, anaemia, hypotension and renal failure. Despite these adverse effects, amphotericin B is still the drug of choice for many serious fungal infections. Recent improved techniques for administering the drug to patients have involved encapsulating amphotericin B in liposomes, e.g. Ambisomes (B) Vester Ltd), which reduces the toxicity, but also reduces the efficacy of the antibiotic. 


\subsubsection{Biosynthesis}

There have been few investigations into the biosynthesis of amphotericin B. An understanding of the factors involved in the assembly of this metabolite in Nature by investigating its biosynthesis may ultimately provide sufficient information for the production of less toxic analogues. The biosynthesis of amphotericin B is thought to occur via the processive pathway, as has been mentioned in previous sections. In amphotericin B, the putative acetate and propionate sub-units can be clearly identified around the polyketide structure. In Fig. 1.22 the structural skeleton of amphotericin B can be seen to be constructed from 17 acetate and 3 propionate units, arising from head-to-tail condensations.

The proposed processive assembly is shown in Fig. 1.23, which is initiated by the condensation of acetyl $\mathrm{CoA}$ (as its enzyme-bound thioester) and propionyl $\mathrm{CoA}$ to give the (2S)-2-methyl-3-oxobutanoyl thioester. Stereospecific reduction of this by a $\beta$-keto reductase will give the desired functionality of the substrate ready for the next condensation. Once the desired chain length is reached, the first enzyme-free

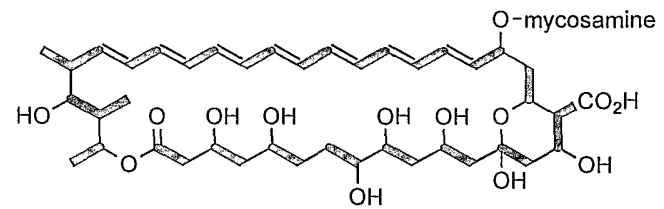

Fig. 1.22 Proposed origin of the carbon skeleton of amphotericin B 


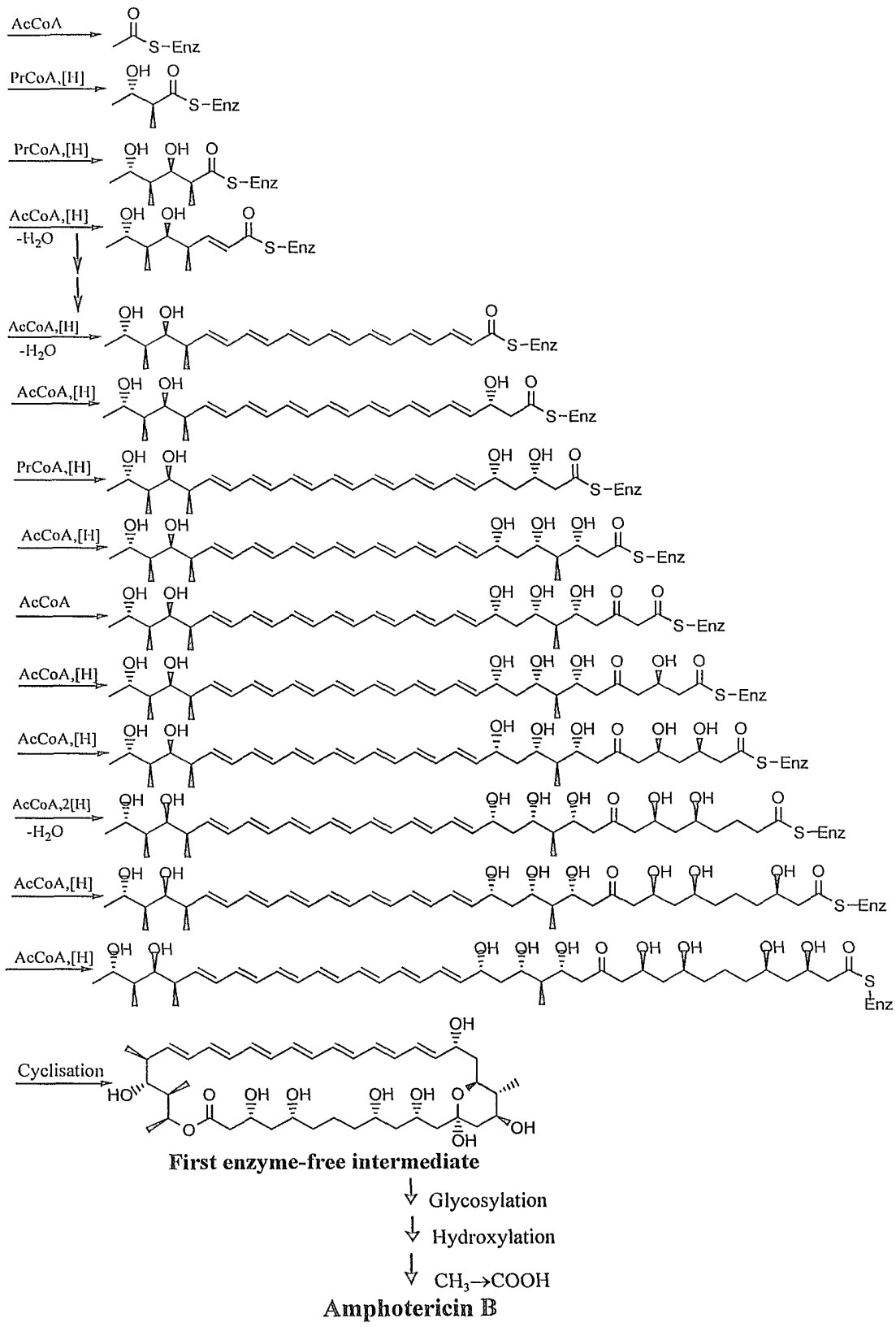

Amphotericin $\mathbb{B}$

Fig. 1.23 Proposed processive biosynthetic pathway of amphotericin B 
intermediate is released as a 38 membered lactone ring. This then undergoes subsequent modifications (hydroxylation, glycosylation and oxidation) yielding the final metabolite. Up to this time, the only biosynthetic studies of amphotericin B have used radioactive labels ${ }^{70-72}$ and no investigations utilising stable isotopes have been undertaken, although there have been investigations into its biological activity, structural conformation by X-ray analysis ${ }^{73,74}$ and its total synthesis. ${ }^{75-78}$

The biosynthesis of amphotericin B in the present study was investigated by a number of methods that have previously been used for the elucidation of biosynthetic pathways for various polyketide metabolites. The three approaches used involved (i) searching for intermediates in the biosynthetic pathway; (ii) the incorporation of labelled acetate; and (iii) the incorporation of a proposed precursor at the diketide stage. The latter approach involved the synthesis of the diketide (3S,2S)-3-hydroxy2- $\left[{ }^{2} \mathrm{H}_{3}\right]$ methyl $\left[{ }^{2} \mathrm{H}\right]$ butanoate $N$-acetylcysteamine $\mathbf{5 1}$ which included strategically positioned deuteriums so as to detect any re-oxidation of the precursor prior to incorporation. The aim of this research was to gain as much information about the biosynthetic processes involved as possible. Further investigations could then be undertaken to locate the genes and enzymes of the PKS to understand its organisation and function, although these genetic aspects were not tackled in this study.

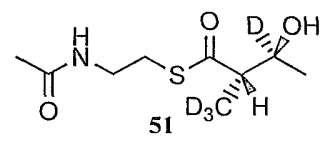




\section{Chapter 2}

\section{Synthesis of target labelled diknetide}

\subsection{Introduction}

As described in the previous chapter, one method of investigating the biosynthesis of polyketides is the attempted incorporation of putative intermediates in the biosynthetic pathway. A general procedure would include the proposal of a biosynthetic pathway, followed by the synthesis of di-, tri- or tetra-ketide intermediates in this pathway, with strategically placed labels to enable intact incorporation to be detected. In Fig. 2.1(a), examples of variously derivatised precursors, as e.g. the $\mathrm{N}$-acetylcysteamine thioesters, can be seen. These have been chemically synthesised and successful incorporation has been detected in a number of studies, e.g. the 'diketide' and 'triketide' incorporated into nargenicin $30^{32}$ and the 'tetraketide' into tetronasin $35{ }^{43}$ From this figure, the functionality in the di-, triand tetra-ketides can be seen to correspond to that in the equivalent intermediate in the biosynthetic pathway. During the chemical synthesis, the required alkyl and hydroxy substituents are incorporated into these precursors, along with isotopic labels, with the desired stereochemistry. 
(a)

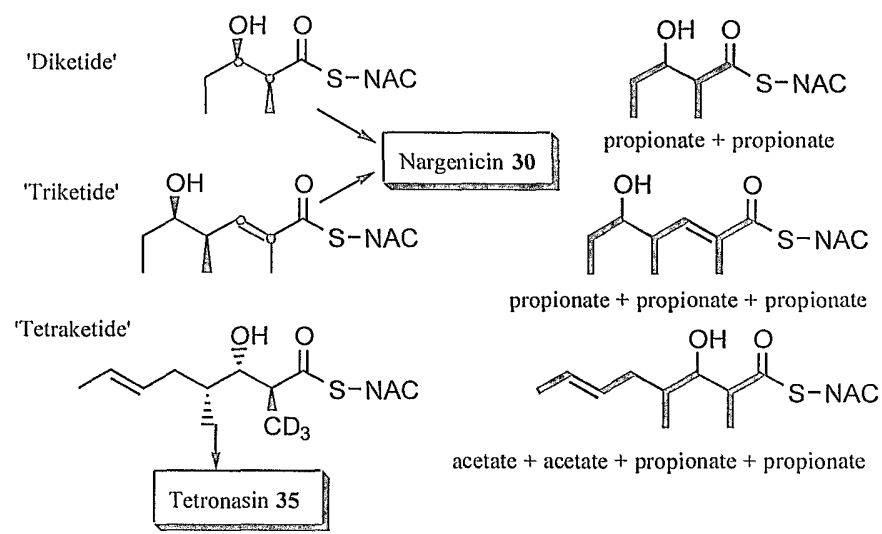

Fig. 2.1 Examples of $(a)$ synthesised precursors, $\circ{ }^{13} \mathrm{C}$; and $(b)$ their substituent subunits

In Fig. 2.1(b) the component units of some previously synthesised precursors are illustrated with, for example, the diketide being made up of two propionate units. There have been a number of examples of diketides of this type having been synthesised in similar studies in which there is an $\alpha$-methyl- $\beta$-hydroxy functionality, comprising of two propionate units. ${ }^{30,31,35}$ However, there have been no examples of precursor incorporation when the diketide is comprised of acetate and propionate units. One reason for this might be due to the more rapid degradation of acetatederived precursors by efficient $\beta$-oxidation, ${ }^{79}$ which can also plague experiments incorporating labelled precursors into the propionate-derived metabolites. To overcome this problem, potential $\beta$-oxidation inhibitors have been used to prevent precursor breakdown, ${ }^{33}$ as was discussed in the Introduction. This study involves the 
first investigation into the biosynthesis of polyenes by the addition of such precursors, in particular the 'acetate + propionate' diketide, the synthesis of which is described in this chapter.

The diketide, with $\alpha$-alkyl and $\beta$-hydroxy functionalities, can exist as a variety of stereoisomers as shown in Fig. 2.2. In recent years there have been a variety of enantioselective and diastereoselective syntheses of $\alpha$-methyl- $\beta$-hydroxy esters/amides/imides etc. ( $\alpha$-substituted $\beta$-keto acid derivatives) as of the type in Fig. 2.2. These have involved the use of chiral auxiliaries, metal and organometallic reagents/complexes and a variety of metal (e.g. boron, lithium) enolates. A range of factors must be taken into consideration when considering the chemical synthesis of such a compound, which will be dealt with in the next sub-section.

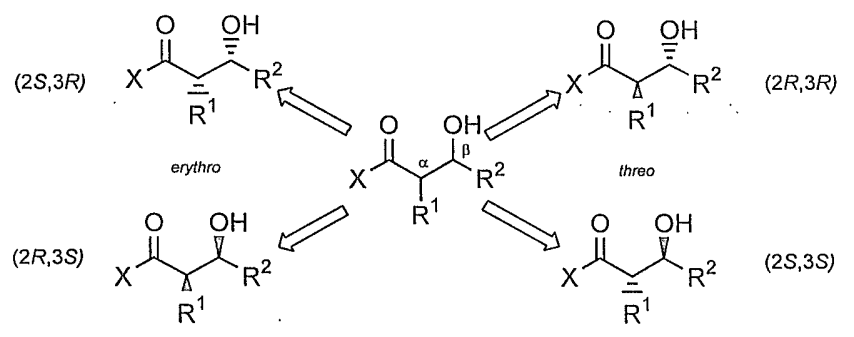

Fig. 2.2 Stereoisomers of $\alpha$-alkyl- $\beta$-hydroxy derivatives 


\subsection{Synthetic strategy}

\subsubsection{Approaches}

The target compound for this chemical synthetic study, (2S,3S)-3-hydroxy-2$\left[{ }^{2} \mathrm{H}_{3}\right]$ methyl $\left[{ }^{2} \mathrm{H}\right]$ butanoyl $\mathrm{N}$-acetylcysteamine thioester 51 , can be seen in Fig.2.3, along with the component acetate and propionate subunits. The NAC thioester derivative was prepared for reasons described in the previous chapter, which principally included the greater ease of trans-thioesterification of the thioester onto the multi-enzyme complex, as opposed to the free carboxylic acid, which is necessary for the incorporation of the precursor into the biosynthetic system of the bacterium.

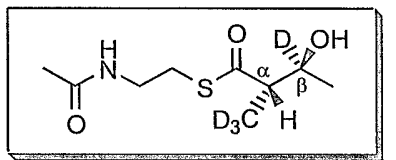

51<smiles>C=C(O)C(=O)C(=O)C#N</smiles>

Fig. 2.3 Target compound with the substituent acetate and propionate subunits indicated

In the proposed biosynthetic pathway of amphotericin B (see Fig. 1.23), there is only one correct stereochemistry at the diketide level, and to accommodate incorporation of the synthetic analogue into the metabolite, the appropriate stereochemistry was incorporated into the target compound 51. The stable isotope labelling pattern that was utilised was chosen with the mode of detection of incorporation (electrospray mass spectrometry) in mind. If the target diketide was incorporated intact, there would be an $\mathrm{M}+4$ peak in the electrospray mass spectra and this would provide evidence for the proposed biosynthetic pathway. However, in this type of study there is often a problem with fatty acid oxidation, which might result in oxidation at the $\beta$ position of the diketide, resulting in the deuterium label being lost. This would thus result in the presence of a peak at $\mathrm{M}+3$ in the electrospray mass spectra, which would not negate the proposed pathway but would confirm the presence of active fatty acid oxidases. 
When designing the synthesis of such a compound there are two general points to take into consideration. The first is the stereochemistry of the 'diketide,' as has been previously mentioned. The second involves the incorporation of the stable isotopic labels into the compound. The criteria for the use of such isotopic labels includes that their relative natural abundance should be extremely low, which is necessary for detection of incorporation into the final metabolite. Other criteria involve a synthetic strategy that is economic and an assay of incorporation that is convenient. Therefore, in this study, readily available sources of deuterium labels (e.g. sodium borodeuteride, deuteriated iodomethane) were considered in the synthesis, which also have the added advantage of being relatively inexpensive.

In recent years there has been much interest in the synthesis of $\alpha$-methyl- $\beta$-hydroxy derivatives as these moieties appear in the framework of many natural products. Stereoselectivity in the synthesis of these derivatives has been challenging and there are now a variety of routes that can be taken. Some possible retrosynthetic routes can be seen in Fig.2.4, without reference to stereochemistry. The diketide could be synthesised by $(a)$ the formation of the $\beta$-keto functionality followed by completely stereoselective methylation and reduction, in either order, or (b) conversely, by incorporation of the deuteriated methyl moiety which is then acylated to give the $\beta$ keto functionality followed by reduction. Alternatively, route $(c)$ involves an 'antialdol' selective reaction to give the hydroxy group directly. After incorporation of the deuteriated methyl group, reaction in an aldol fashion would give the required $\beta$ hydroxy functionality. 


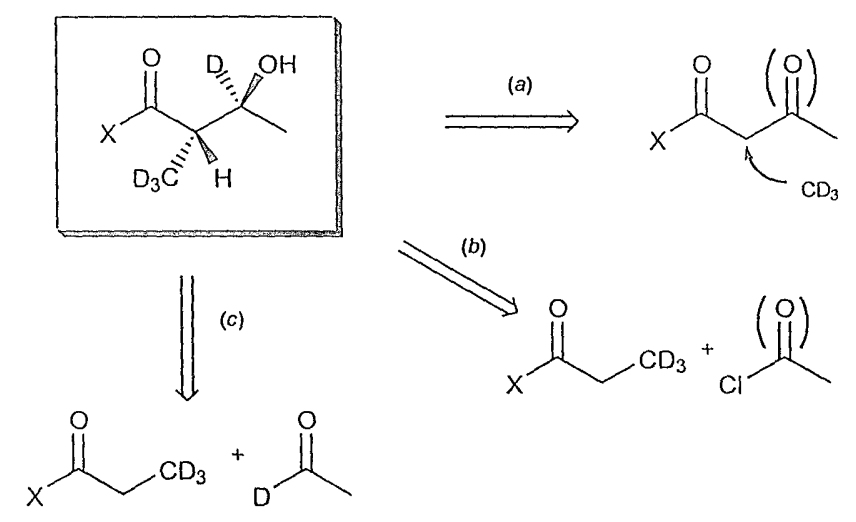

Fig. 2.4 Retrosynthetic analysis of the target compound

One of the paths that has been taken in recent years, that was of great interest to us initially, was the work of Frater and co-workers. This work involved the highly stereoselective $\alpha$-alkylation of $\beta$-hydroxy esters (Scheme 2.1). ${ }^{81}$ This reaction was consequently believed to have such 'acyclic stereoselection, ${ }^{\prime 80}$ due to the rigid cyclic intermediate 54 that is formed due to the chelation of a lithium cation with the two O-anions, ${ }^{81}$ although this may be an over-simplification. ${ }^{82}$ For this reaction, the initial enantiopure $\beta$-hydroxy ester was formed by reduction with (fermenting) Baker's yeast (Saccharomyces cerevisiae). ${ }^{83}$ Such reductions have been used previously for studies on the chemical synthesis of amphotericin $B{ }^{84}$ and for a variety of other synthetic studies of natural products. ${ }^{85}$ Unfortunately this stereoselective reduction was not applied in the present synthesis, because it would have been necessary to carry out the fermentation process in $\mathrm{D}_{2} \mathrm{O}$ for the incorporation of the necessary labels, which would be expensive. 


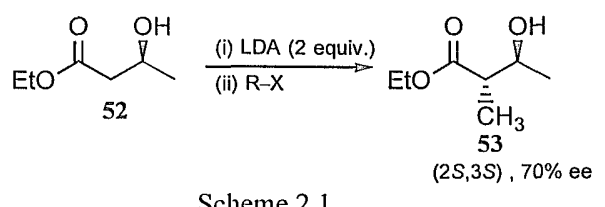

Scheme 2.1

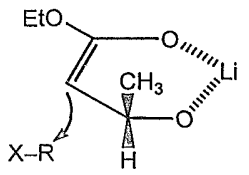

54

An alternative chemical assembly includes ethylation of the $\beta$-keto ester which could subsequently be diastereoselectively reduced, as seen in Scheme 2.2. ${ }^{86}$ Preliminary reactions of this type, but involving methylation under similar conditions, have been previously attempted in our group, however no satisfactory results were obtained. The main problem encountered involved the reactions producing a mixture of monoand di-alkyated products. Purification also proved difficult as their separation involved distillation techniques which would need to be carried out on a large scale. As we would be working with expensive labels, this approach was not investigated further. These problems were also encountered by other researchers. ${ }^{86}$

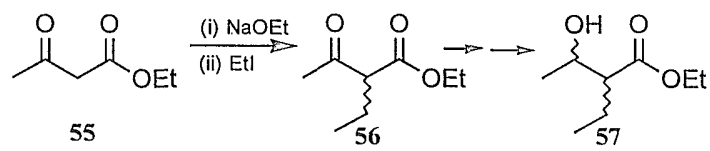

Scheme 2.2 
Using a slight variation of work mentioned previously, Frater et al. then went on to investigate the production of $(2 R S, 3 R S)$ - $\alpha$-alkyl- $\beta$-hydroxy esters using a one-pot aldol alkylation method, which was suggested to proceed via the species as shown in Scheme 2.3. ${ }^{81}$ This reaction also turned out to be diastereoselective in favour of the $(2 S, 3 S)$ isomer 62. Thus, incorporation of the desired labels with this procedure would involve the reaction with deuteriated iodomethane and also deuteriated acetaldehyde, which is commercially rather expensive, and although it can be chemically synthesised, a more readily available source of labels was sought.

Another approach to achieve the target compound would be to first form the correct stereocentre by chemical reduction at the hydroxy moiety rather than enzymically via yeast. One novel study that was considered involved the use of optically active sulfoxides $(63$, Scheme 2.4$){ }^{87}$

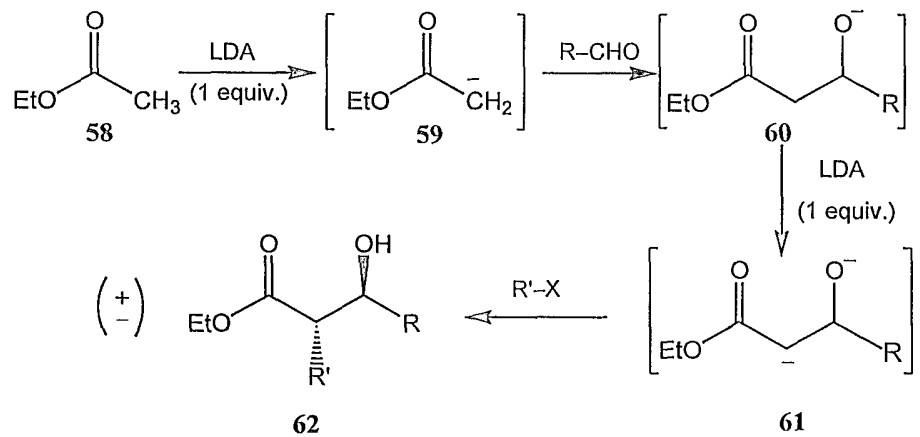

Scheme 2.3 

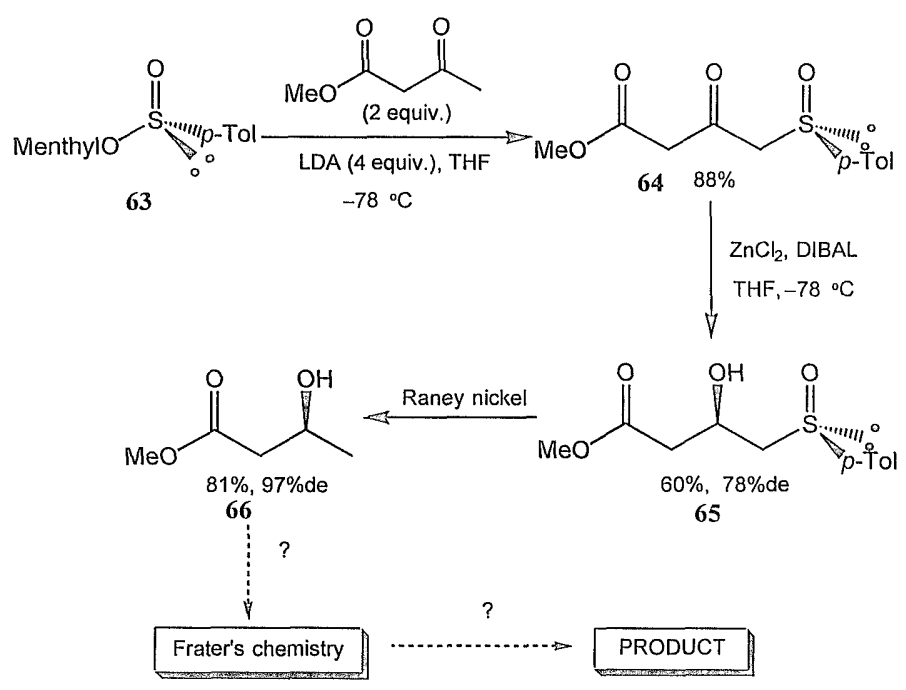

Scheme 2.4

This approach would enable an asymmetric synthesis of the $\beta$-hydroxy derivative, with the incorporation of deuterium occurring with the use of deuteriated diisobutylaluminium hydride, which is commercially available, although slightly more expensive than other deuteriated reducing agents. From the product of these reactions, the procedure could have been taken one step further using the Frater chemistry on a small scale, using deuteriated iodomethane for the incorporation of the other labels. This synthetic route appears to be viable, and is, with hindsight, certainly one route that could have been chosen. 
An alternative approach was then considered which was mentioned at the retrosynthetic stage [route $(c)$ ] which involves aldol condensations. Reaction of a ketone with an aldehyde in the presence of strong base would result in the $\beta$-hydroxy ketone 69 as shown in Scheme 2.5. This reaction can be made regioselective because the first step of the mechanism involves the formation of an enolate by the removal of an $\alpha$-hydrogen. With an unsymmetrical ketone there are two possible enolates that can be formed (cis or trans) with the precedence of either form depending on the base used and the conditions employed; varying these two conditions will result in either the thermodynamic enolate or the kinetically favoured enolate being produced.

The diastereoselection observed with aldol reactions has been of interest for many years, with the first explanation of such reactions being given in the 1950 s. $^{88}$ Later investigations $\mathrm{s}^{89,90}$ showed that kinetic aldol condensations were indeed defined by

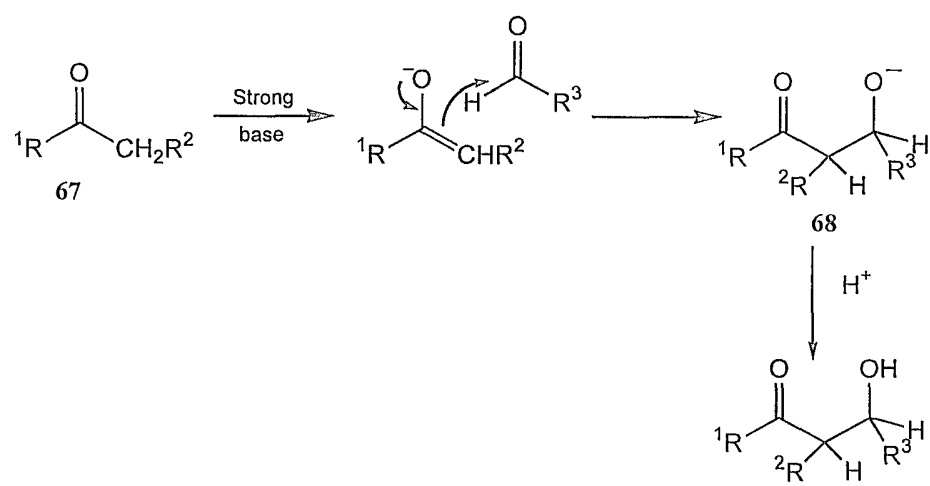

Scheme 2.5

69 
enolate geometry and that, with chair-type transition states, cis enolates generate the erythro products, whilst the trans enolates exhibit the threo products (Scheme 2.6). The control of enolate configuration or the use of preformed enolate derivatives provide a way to control the stereoselectivity of the aldol condensation. The reaction creates two new chiral centres, and in the most general case, there are four stereoisomers which can be seen in Fig.2.5. In the present study it is the threo product that is required and this reaction would have to undergo an anti aldol reaction via a trans enolate. Much progress has been made in the past ${ }^{91,92}$ in both the control of the diastereoselection of this reaction (Fig 2.5; E1+E2 vs. $T 1+T 2$ ) and of the enantioselection ( $E 1 \quad v s . E 2$ or $T 1$ vs. T2). This has been done by the use of various enolates of lithium, zinc and boron, to name but a few.

It was shown that the use of strong base, such as LDA, on standard alkyl esters produced the trans enolate, but (due to low kinetic selectivity) a ratio of $1: 1$ of the threo isomers was produced. However the reaction for bulky aryl esters [e.g. dimethylphenyl (DMP)] can be used to give good anti selectivity due to diastereofacial selection (Scheme 2.7) ${ }^{93}$ Under certain conditions, both zinc and boron enolates have been demonstrated to produce a favourable ratio of threo:erythro products. ${ }^{94,95}$ However, the two enantiomeric products are still formed and so to make the reaction enantioselective, a chiral enolate would be required. This was achieved by Masamune $e t$ al. with the use of a chiral boron reagent which proved to be essentially enantioselective (Scheme 2.8). ${ }^{96}$ 


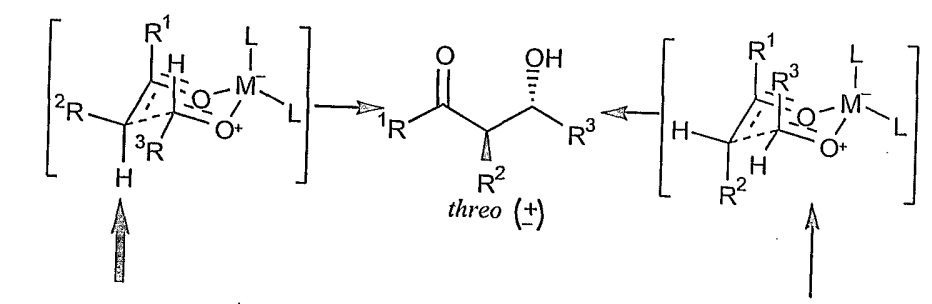

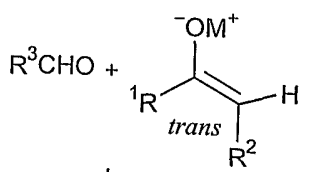

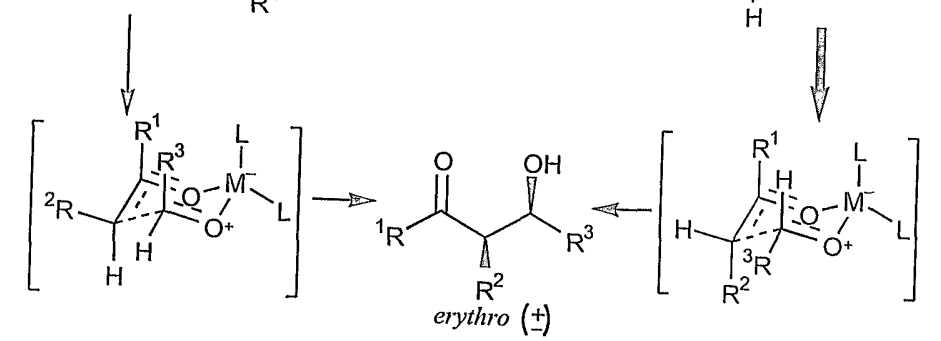

Scheme 2.6

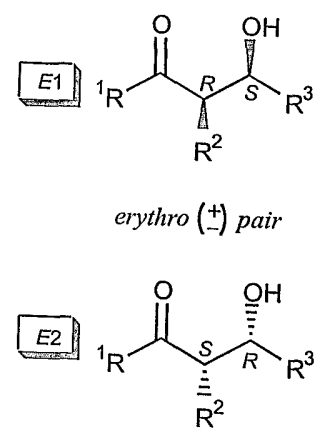<smiles>[R]C(=O)C([R])C([R])O</smiles>
threo (†) pair<smiles>[R]C(=O)C([R])C(O)[R]C1C=CC1</smiles>

Fig 2.5 The four stereoisomers possible with the creation of the two chiral centres 


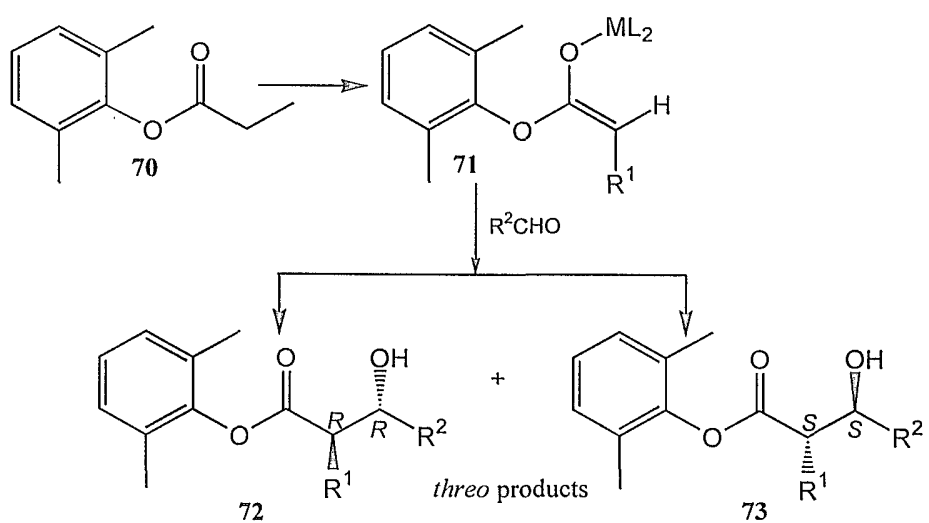

Scheme 2.7

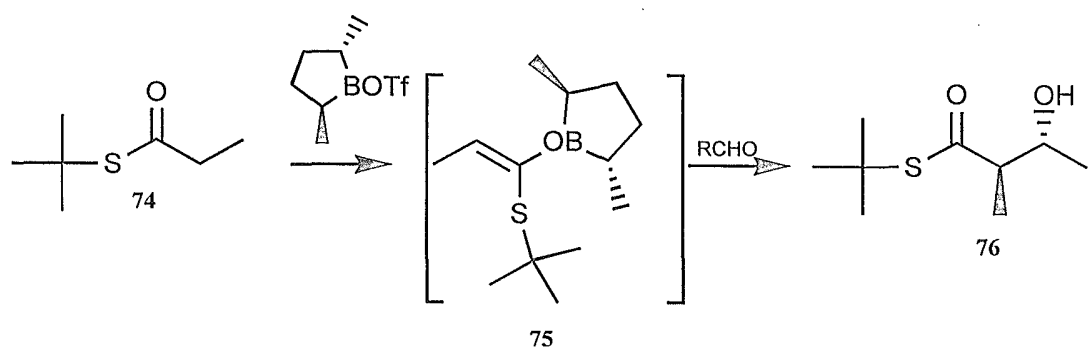

Scheme 2.8 
Many other chiral enolates have been used with high stereoselectivities in aldol condensations, ${ }^{97,98}$ however in investigations in which the threo isomer was produced instead of the erythro isomer, the $R, R$ enantiomer was produced in most circumstances, rather then the $S, S$.

Other chiral auxiliaries have also been used to produce chiral enolates. Evans et al. have developed an enantioselective aldol condensation involving chiral oxazolidin-2ones. $^{99}$ In these reactions (Scheme 2.9), even though the lithium and boron enolates that are formed are of the $Z$ form, it is actually the erythro products that are produced. Therefore the chiral auxiliary is influencing the reaction in such a manner as to produce different products via the $Z$ enolates. This methodology has been utilised in a variety of cases for the synthesis of precursors in biosynthetic studies. In the case of nargenicin, the diketide is comprised of two propionate subunits, which is synthesised by a syn aldol methodology via a boron enolate (dibutylboron triflate). ${ }^{32}$ Such reactions were also carried out for the synthesis of the tetraketide for incorporation into tetronasin. ${ }^{100}$

It was decided that even though a suitable route may be found for the target compound via an aldol condensation pathway, it did not appear that the desired threo isomer could be produced in a favourable ratio, and so a different pathway was 


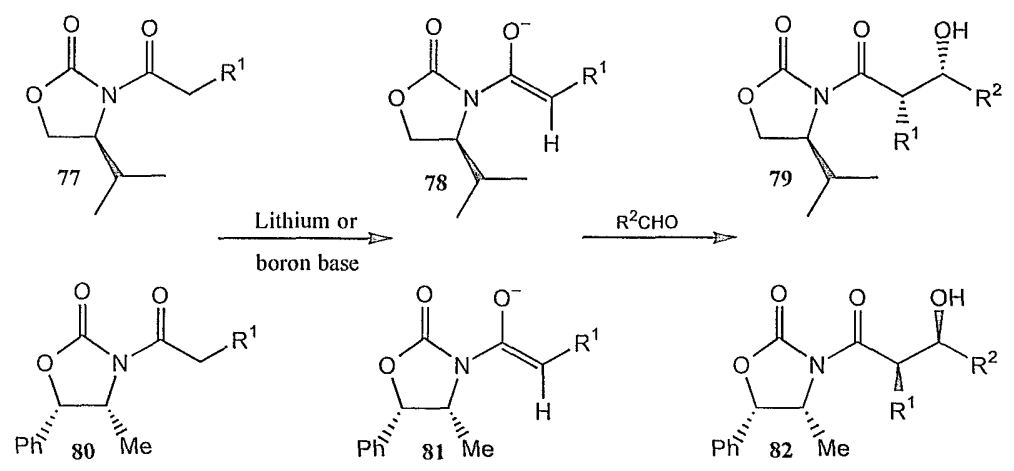

Scheme 2.9

sought. Also whilst the aldol reaction between a propionate enolate and propanal was diastereoselective, it was not known to be for a propionate enolate and acetaldehyde. Also, deuterio labelled acetaldehyde would be required, which is a volatile liquid and so not the most desirable labelled reagent. Deuterioacetaldehyde 86 is commercially available but expensive. It can be synthesised by addition of $D_{2} \mathrm{O}$ to the anion of the thioacetal of acetaldehyde (Scheme 2.10) but isolation and purification of the volatile labelled product $\left(\mathrm{bp} \sim 21^{\circ} \mathrm{C}\right)$ would be difficult on a small scale, therefore this option was not taken any further.

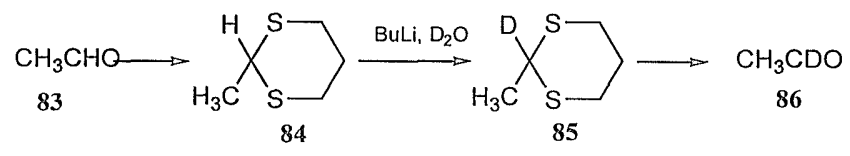

Scheme 2.10 
Although this option was discounted, the concept of using chiral auxiliaries was not. Indeed, it was found that when the chiral oxazolidin-2-ones and various metal enolates were used in a series of reactions, the result was a highly enantioselective route to the required product. As well as the previously mentioned aldol condensations, the chiral auxiliary oxazolidin-2-one was also used in asymmetric alkylation reactions for the preparation of carboxylic acid derivatives, ${ }^{101}$ in halogenations, ${ }^{102}$ hydroxylations ${ }^{103}$ and in asymmetric acylation reactions for the construction of chiral $\beta$-dicarbonyl synthons, ${ }^{104}$ the latter of which was of interest in our synthetic strategy. If the $Z$ enolate 87 is reacted with an appropriate acid chloride the corresponding isomer of the $\beta$-ketoimide 88 will be produced (Scheme 2.11). This route affords the correct stereochemistry at the $\alpha$-position in a simple fashion and would allow the incorporation of labels at this position by the use of the readily available deuteriated iodomethane. The label at the $\beta$-position could then be incorporated by reduction with deuteriated sodium borohydride (or an equivalent reducing agent) to give two diastereoisomers, which could then be separated. Thus this route was chosen for the availability and cost of the labels, as well as providing access to both the $S, S$ and $S, R$ enantiomers of the $\alpha$-methyl- $\beta$-hydroxy derivative; the separated $S, S$ diastereoisomer could then be converted into the corresponding desired NAC thioester analogue. 


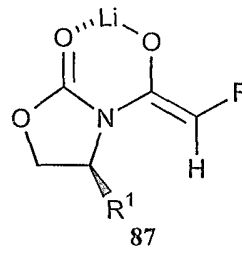

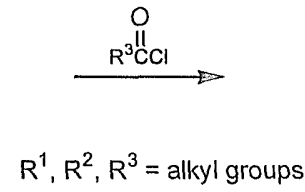

Scheme 2.11

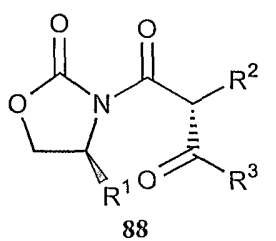

88

\subsubsection{Clnemical symthesis}

\subsubsection{Methodology: (S)-Valine route}

The first step in the pathway to produce the target compound 51 involved the synthesis of the chiral auxiliary. Various oxazolidin-2-ones have been used in numerous asymmetric reactions, in particular the 4-methyl-5-phenyl- $89,{ }^{99,105} 4$ phenylmethyl- $90^{106}$ and 4-isopropyl-oxazolidin-2-ones $91 .^{107,108}$ These have been utilised because of the availability of the precursors and, more interestingly, the chirality they induce into the tetrahedral centres contained in the alkyl or acyl chains attached to the nitrogen via ketoimides, which will be discussed in more detail later.
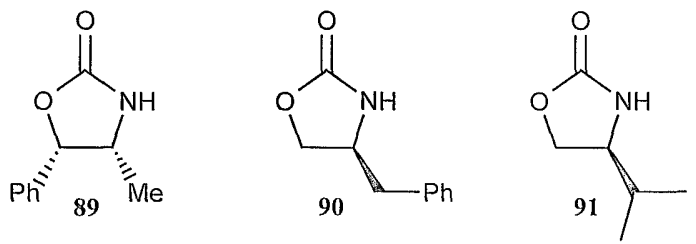
In studies carried out by Evans et al. ${ }^{109}$ it was found that the (S)-4isopropyloxazolidin-2-one when converted to the imide enolate 92 underwent a highly diastereoselective acylation to give the product isomer $\mathbf{9 3}$ as shown in Scheme 2.12. After reduction, this pathway would thus lead to eventually give the opposite enantiomer of our target compound, with the methyl bearing chiral centres having the $R$ orientation instead of the $S$.

For the synthesis of our target compound 51, the use of the enantiomeric $(R)-4$ isopropyloxazolidinone as the chiral auxiliary would therefore be required, which is prepared from $(R)$-valine. However, as this is the more expensive of the two enantiomers, it was decided to carry out a series of model reactions initially using the $(S)$-valine-derived compounds, in order to investigate conditions etc. prior to the use of labels. In the synthetic route to the chiral auxiliary, the amino acid valine first needs to be reduced to valinol (Scheme 2.13). This, and other similar reductions of

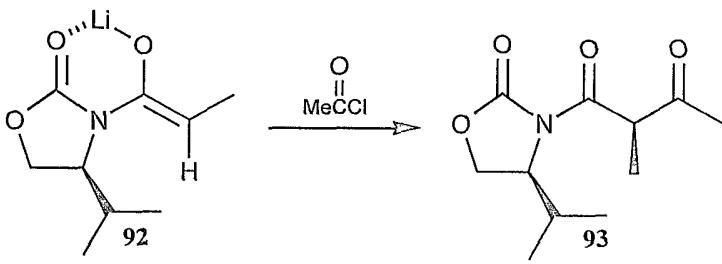

Scheme 2.12 


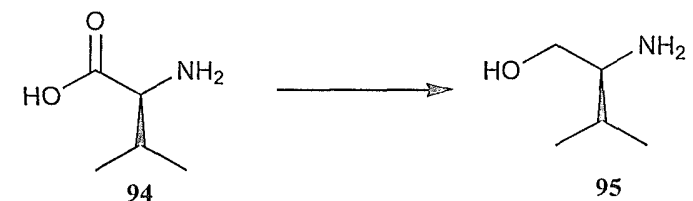

Scheme 2.13

other $\alpha$-amino acids have been carried out by a variety of methods. ${ }^{110,111}$ The first one that we attempted involved the reduction of $(S)$-valine via the boranedimethylsulfide- $\mathrm{BF}_{3}$ complex. ${ }^{12,113,114}$ This reaction takes place with the liberation of the odorous and toxic dimethyl sulfide and only results in a product yield of $c a .40 \%$. An improved method involved trimethylsilyl chloride (TMSCl) and lithium borohydride in THF. ${ }^{115}$ In this reduction it is proposed that a borane-THF complex is formed which, with the assistance of an excess of TMSCl, acts as the reducing agent [eqn. (1)]. This procedure is much more expedient overall and produces $(S)$ valinol 95 in $78 \%$ yield (Scheme 2.14). The resulting $\beta$-amino alcohol was converted to the corresponding 2-isopropyloxazolidinone by reaction with diethyl carbonate in toluene and a catalytic amount of sodium methoxide. ${ }^{116}$ The reaction mixture was heated at reflux for $c a .5 \mathrm{~h}$ and the ethanol that was produced as a byproduct was occasionally distilled off; compound 91 was obtained in $78 \%$ yield.

$\mathrm{LiBH}_{4}+\mathrm{Me}_{3} \mathrm{SiCl} \stackrel{\mathrm{THF}}{\longrightarrow} \mathrm{LiCl}+\mathrm{Me}_{3} \mathrm{SiH}+\mathrm{BH}_{3} \cdot \mathrm{THF}$ 


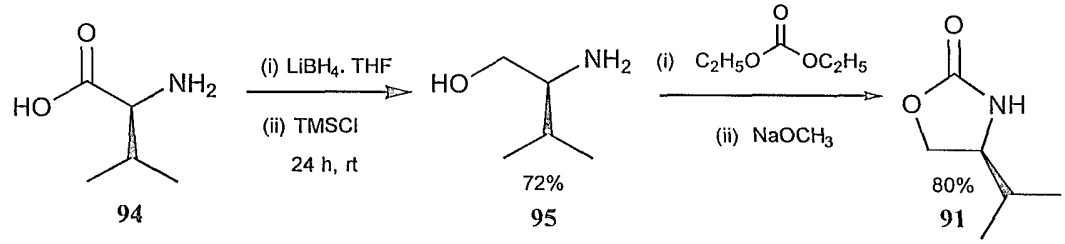

Scheme 2.14

The general scheme of reactions to form the $\beta$-ketoimide 93 can be seen below (Scheme 2.15). In order to investigate all the pathways, $N$-propionyloxazolidinone 92 was first prepared [in $70-75 \%$ yield; path $(a)$ ] which would then be acylated to give the product 93 . However, for the incorporation of deuterium labels into the appropriate positions, the $N$-acetyloxazolidinone had to be prepared, which would then be reacted with $\mathrm{CD}_{3} \mathrm{I}$ for the incorporation of the label. Thus, pathway $(b)$ was also to be investigated in which 91 underwent lithiation (BuLi, THF) with subsequent reaction with acetyl chloride to form $\mathbf{9 6}$, which was prepared in good yields $(70-75 \%)$.

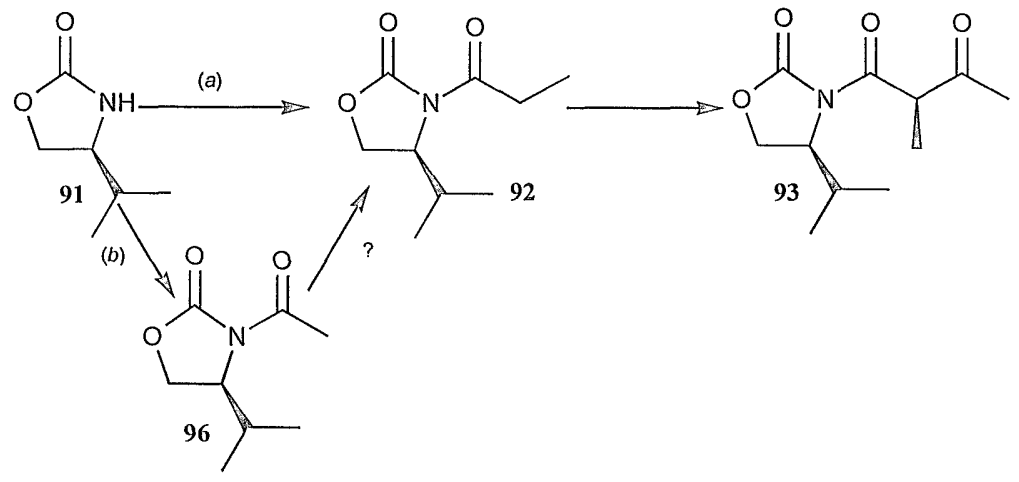

Scheme 2.15 
The methylation of compound $\mathbf{9 6}$ to give $\mathbf{9 2}$ however proved troublesome. For this alkylation and subsequent acylation reactions, the formation of the enolate is of utmost importance. Transformation of the alkylimides to their corresponding enolates is carried out in the presence of lithium, sodium or boron bases, such as lithium diisopropylamide $\left[\mathrm{LDA}, \operatorname{LiN}\left(\mathrm{Pr}^{i}\right)_{2}\right]$ or sodium hexamethyldisilylazide $\left[\mathrm{NaN}\left(\mathrm{SiMe}_{3}\right)_{2}\right]$ at $-78{ }^{\circ} \mathrm{C}$ in THF. It has been found that the reactions of these enolates show greater diastereoselection and because of the stereoselectivity of the reactions, it was deduced that the chelated enolates must be of the $Z$ form (Fig. 2.6) with a greater than 100:1 ratio over the $E$ form. ${ }^{101}$ Thus, in our study the alkylation reaction was initially attempted with sodium hexamethyldiilylazide (1.1 equiv., -78 ${ }^{\circ} \mathrm{C}$, THF) by which 96 was transformed into the metal enolate, although yields of the product were low $(<10 \%){ }^{99}$

The alkylation reaction via the metal enolate in Fig. 2.6 exhibits high levels of diastereoselection when $\mathrm{R}^{2}$ is a methyl or an ethyl group and also when the alkyl halide is sterically bulky (i.e. $\mathrm{PhCH}_{2} \mathrm{Br}$ is more diastereoselective than $\mathrm{MeI}$ ). In

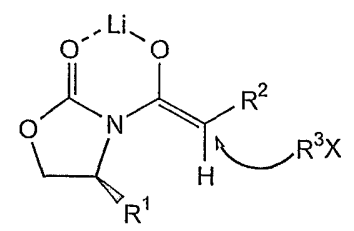

Fig. 2.6 Alkylation reaction involving the $Z$ lithium enolate; $\mathrm{R}^{1}, \mathrm{R}^{2}$ and $\mathrm{R}^{3}=\mathrm{H}$ or alkyl, $\mathrm{X}=$ halide 
The methylation of compound 96 to give 92 however proved troublesome. For this alkylation and subsequent acylation reactions, the formation of the enolate is of utmost importance. Transformation of the alkylimides to their corresponding enolates is carried out in the presence of lithium, sodium or boron bases, such as lithium diisopropylamide $\left[\mathrm{LDA}, \mathrm{LiN}\left(\mathrm{Pr}^{\mathrm{i}}\right)_{2}\right]$ or sodium hexamethyldisilylazide $\left[\mathrm{NaN}\left(\mathrm{SiMe}_{3}\right)_{2}\right]$ at $-78{ }^{\circ} \mathrm{C}$ in THF. It has been found that the reactions of these enolates show greater diastereoselection and because of the stereoselectivity of the reactions, it was deduced that the chelated enolates must be of the $Z$ form (Fig. 2.6) with a greater than 100:1 ratio over the $E$ form. ${ }^{101}$ Thus, in our study the alkylation reaction was initially attempted with sodium hexamethyldisilylazide (1.1 equiv., -78 ${ }^{\circ} \mathrm{C}$, THF) by which 96 was transformed into the metal enolate, although yields of the product were low $(<10 \%) .{ }^{99}$

The alkylation reaction via the metal enolate in Fig. 2.6 exhibits high levels of diastereoselection when $\mathrm{R}^{2}$ is a methyl or an ethyl group and also when the alkyl halide is sterically bulky (i.e. $\mathrm{PhCH}_{2} \mathrm{Br}$ is more diastereoselective than $\mathrm{MeI}$ ). In

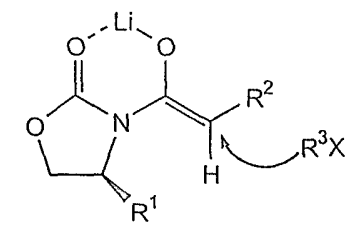

Fig. 2.6 Alkylation reaction involving the $Z$ lithium enolate; $\mathrm{R}^{1}, \mathrm{R}^{2}$ and $\mathrm{R}^{3}=\mathrm{H}$ or alkyl, $\mathrm{X}=$ halide 
such reactions it is relatively easy to form an enolate as there is an internal double bond which is thermodynamically stable. The diastereoselectivity of this reaction is not an issue as $\mathrm{R}^{2}=\mathrm{H}$ (as in 96), but this may be the reason for the low yields of this reaction, as the enolate is not as thermodynamically stable as the $N$-propionyl derived enolate. Thus, varying the reaction conditions, including the use of a different base and solvent systems, was attempted in order to increase the yield. Methylation involving the sodium base was carried out in THF and required a temperature of -78 ${ }^{\circ} \mathrm{C}$ to be maintained for the duration of the reaction ( $c a .6-10 \mathrm{~h}$ ) as sodium enolates can be unstable above $-20^{\circ} \mathrm{C}$. As the reaction was run overnight it proved difficult to maintain the required temperature, which may also have been a contributing factor to the low yields of this reaction. Thus, enolate formation using a lithium base was attempted, even though it was the sodium base that had been preferable for previous enolate methylations. ${ }^{101}$ Initial attempts with lithium diisopropylamide as a base involved formation of the enolate and addition of methyl iodide at $-78^{\circ} \mathrm{C}$ but the reaction mixture was then allowed to warm to $0^{\circ} \mathrm{C}$ and held at that temperature for a further $4 \mathrm{~h}$ (lithium enolates decompose above $0^{\circ} \mathrm{C}$ ). However, these attempts also gave low yields $(<20 \%)$, which were far from comparable with those in the literature $(\sim 70 \%){ }^{101}$

It is known that changes in the solvent can have a beneficial effect on the rate of various reactions, e.g. enolisations and subsequent acylations. ${ }^{141}$ Certain polar aprotic solvents, e.g. hexamethylphosphoric triamide 97 (HMPA), can have an effect on the solubility of certain compounds and they also have the ability to solvate 
cations. The solubility of the enolate has been shown to be increased by changing the solvent system from THF to THF-HMPA. ${ }^{17,118}$ Formation of the enolate is influenced by the solvation effects present when HMPA is used in the presence of LDA. ${ }^{11}$ It has been known for some years now, however, that HMPA is a carcinogen $^{120,121}$ and so a safe substitute was used in the form of the propylene urea DMPU 98 [1,3-dimethyl-3,4,5,6-tetrahydro-2-(1H)-pyrimidinone]. ${ }^{122}$ However when DMPU was employed as a co-solvent in the attempted conversion of 96 to 92 , initially no increase on the

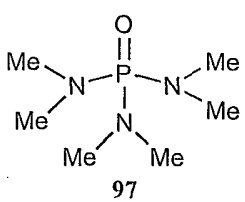<smiles>CN1CCCN(C)C1=O</smiles>

98

previous yield could be achieved. It is known that this alkylation is very sensitive to any moisture present ${ }^{141}$ and every effort was made to ensure a moisture-free environment (distilling DMPU, $\mathrm{AcCl}$, etc.). Subsequent investigations showed that that the most important step in the reaction was the rate of warming of the reaction mixture to $0{ }^{\circ} \mathrm{C}$, after addition of methyl iodide to the lithium enolate at $-78{ }^{\circ} \mathrm{C}$. It seems that if this occurs at too fast a rate, there is a solidification of the white reaction mixture. Thus, when this procedure was carried out using THF-DMPU $(50: 50, v / v)$ and with a slow warming to $0{ }^{\circ} \mathrm{C}$, the formation of the enolate and its methylation occurred and after work up and purification of the crude product by flash column chromatography, 92 was obtained in $33 \%$ yield based on recovered starting material (Scheme 2.16). Further attempts to increase the yield were not attempted. 

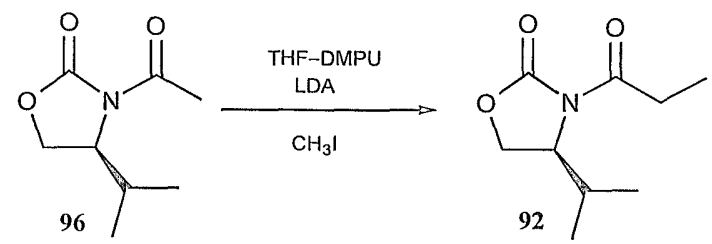

Scheme 2.16

The next step was the preparation of the $\beta$-ketoimide 93 . The procedure of Evans et $a l .{ }^{109}$ involves rapid addition of the precooled enolate to a cool solution of the acyl chloride in THF, followed by an immediate quenching with ammonium chloride. However, when this acylation was attempted, very low yields of product resulted. Thus, following a modification of this procedure formulated within our group, the chiral $N$-propionyloxazolidinone 92 was transformed into the $Z$ lithium enolate with lithium diisopropylamide at $-78^{\circ} \mathrm{C}$. Acetyl chloride in THF at $0{ }^{\circ} \mathrm{C}$, was then added to this solution and the reaction stirred at $-78{ }^{\circ} \mathrm{C}$ for $30 \mathrm{~min}$ before being quenched with saturated aqueous ammonium chloride to give the product as a single diastereoisomer 93 , after purification by flash column chromatography, in $57 \%$ yield (or $76 \%$ based on recovered starting material) (Scheme 2.17).<smiles>CCC(=O)N1C(=O)OCC1C(C)C</smiles>

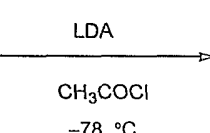

$-78{ }^{\circ} \mathrm{C}$<smiles>C=C(C(=O)N1C(=O)OC[C@H]1C(C)=O)C(C)C</smiles> 
Fig.2.7 shows the 'H NMR spectrum of the purified, crystalline product, showing the clean $3 \mathrm{H}$ doublet at $\delta 1.39$, corresponding to the three hydrogens of the methyl on the carbon at the $\alpha$-position, with no presence of the other diastereoisomer. In the NMR spectrum, the signal for the methine hydrogen (between the two exocyclic carbonyls) can be seen to be shifted downfield quite substantially (to $\delta 4.53$ ) compared with the methyl of the starting material $(\delta 2.93)$ which can be explained by the deshielding of the additional carbonyl.

The diastereoselectivity of these reactions can be explained by the transition state steric effects imposed by the $R$ substituent of the oxazolidinone (in this case isopropyl, Fig. 2.8), which determines the $\pi$ facial stereoselection of the $Z$ lithium enolate. It would be expected that the methine hydrogen $\left(\mathrm{H}_{\alpha}\right)$ between the carbonyls would be rather acidic $\left(\mathrm{p} K_{\mathrm{a}} 10-13\right)$ and the newly created asymmetric centre in this $\beta$-keto imide would be readily lost through enolisation. However, this is not the case and in fact these compounds have very low kinetic acidity and are quite stable to silica gel chromatography. One possible explanation for the low kinetic acidity might be associated with $\mathrm{A}(1,3)$ strain conformation effects. ${ }^{123}$ An X-ray structure of 93 (Fig. 2.8) showed that due to the steric effects of the surrounding oxazolidinone and methyl substituents, the $H_{\alpha}$ is induced to lie orthogonal to the $\pi$ systems of both the adjacent carbonyls. Interactions between the methyl substituents and the imide nitrogen substituents of the chiral auxiliary appear to reduce the influence of the 


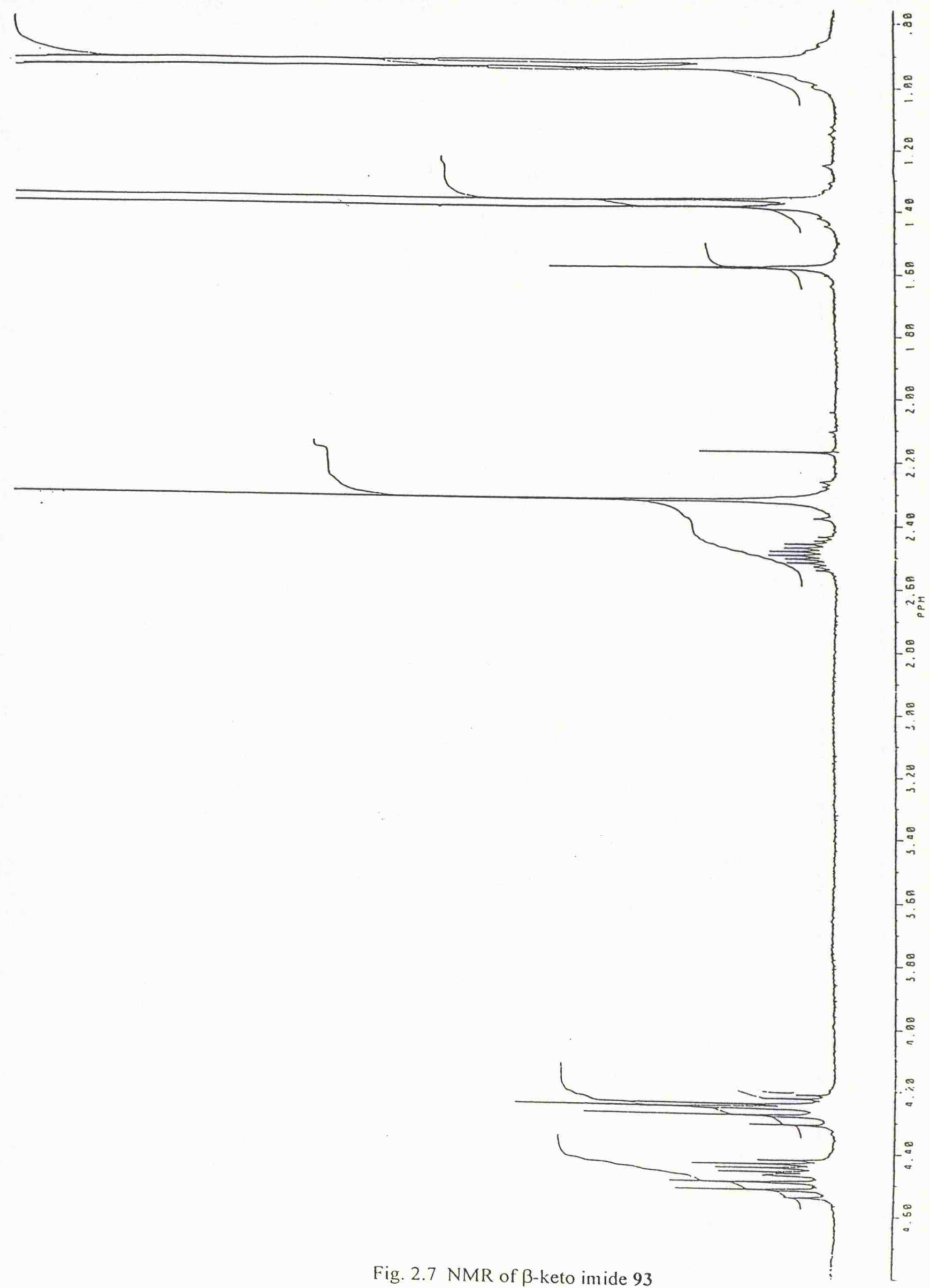

Fig. 2.7 NMR of $\beta$-keto imide 93 

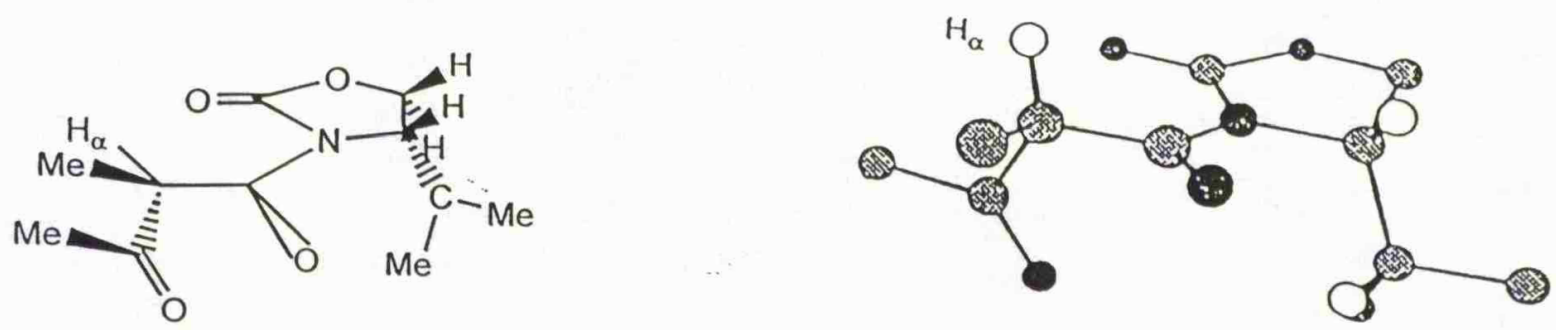

Fig.2.8 Molecular modelling representation of compound 93

exocyclic imidic carbonyl toward $\mathrm{H}_{\alpha}$ acidification, by destabilising the conformation in which the $\mathrm{H}_{\alpha}$ lies parallel to the carbonyl $\pi$ system. ${ }^{104}$ For a proton $\alpha$ to the $\mathrm{C}=\mathrm{O}$ bond to be acidic, it would have to be perpendicular to the $\pi$ system.

The next reaction involved the ideally completely stereoselective reduction of the $\beta$ keto group. In this reaction it was important to use a method which conveniently incorporated the desired deuterium label. There have been a variety of reagents used to produce different ratios of the diastereoisomeric products 99 and 100 (Scheme 2.18).<smiles>CC(=O)[C@H](C)C(=O)N1C(=O)OC[C@H]1C(C)C</smiles>

Scheme 2.18 
Results from experiments involving the reduction of $\beta$-keto derivatives of esters, acids or amides etc., as well as the $\alpha$-alkylated- $\beta$-keto derivatives, have been useful for suggesting the conditions to be used. Reductions involving $\alpha$-amino ketones give us some idea of the ratio of diastereoisomers produced, which can be varied by the use of different reagents. ${ }^{124}$

Now considering $\alpha$-substituted- $\beta$-keto esters or amides derivatives, it appears that there are a number of procedures which although diastereoselective, unfortunately give predominantly the erythro product. ${ }^{109,125,126}$ These include reduction with the non-commercially available and pyrophoric $\mathrm{Zn}\left(\mathrm{BH}_{4}\right)_{2}{ }^{127}$ and also with hydrosilanes in acidic media. ${ }^{128}$ If the reaction conditions of the latter are altered slightly however (reduction in the presence of $\mathrm{F}^{-}$instead of $\mathrm{H}^{+}$), the selectivity can be reversed, with the threo product resulting. ${ }^{129}$ There have been fewer examples of diastereoselective reduction to give the threo product predominantly, but certain conditions do achieve this. With all these reactions in mind, the reduction in this study was considered.

As already mentioned, $\mathrm{Zn}\left(\mathrm{BH}_{4}\right)_{2}$ has been used in asymmetric reduction to afford the erythro product, as can be seen in Scheme 2.19. ${ }^{130}$ An important factor in the erythro selective reduction was the intermediary formation of a six-membered metal chelate (Fig. 2.9). Therefore for the threo selective reduction, it seemed that the use of metal hydride complexes having counter ions of high O-chelating ability were to be avoided. Furthermore, in order to eliminate complexity due to the disproportionation of the reducing species, reducing agents of choice would have complexed hydride 
anions of less dissociative nature, with the anions having adequate bulkiness to discern the diastereoisomerically different environments. It was found that potassium triethyl-borohydride fitted these requirements and indeed the reduction was shown in the literature ${ }^{130}$ to proceed to give the corresponding threo hydroxy

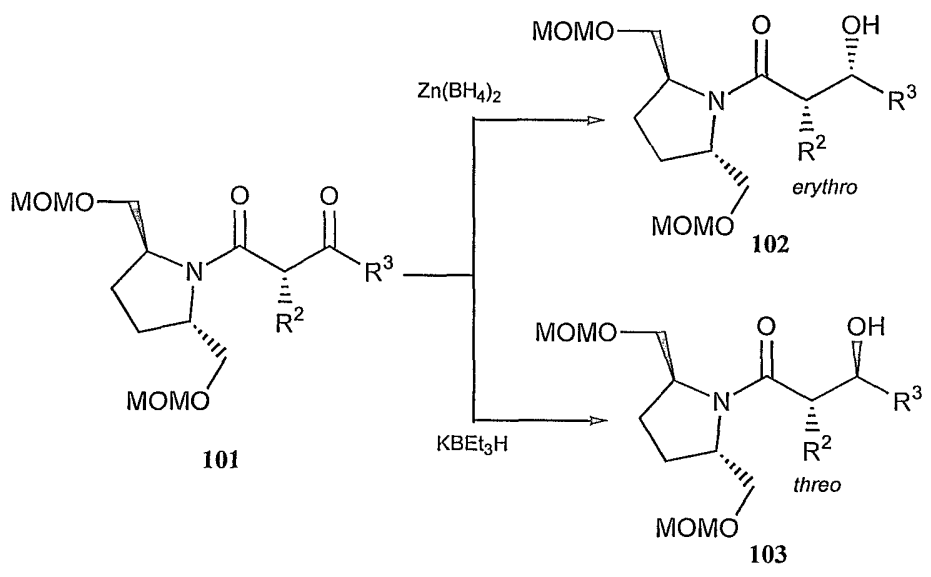

Scheme 2.19; MOM = methoxymethyl

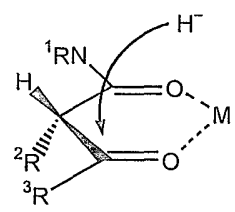

Fig. 2.9 Erythro inducing six-membered metal chelate 
amide with high diastereoselectivity. The threo selectivity may be explained by Felkin-Ahn's model where $\sigma^{*}-\pi^{*}$ interactions stabilise the transition state (Fig. 2.10), and as such a conformation is considered to be particularly favoured when substituents on the amide nitrogen are bulky because the $A(1,3)$ interaction is minimised therein. As a result of the bulky group attached to the amide nitrogen, the hydride ion attacks the $\beta$-carbonyl at the opposite side exclusively (X-ray analysis has shown that the oxazolidinone $\beta$-keto imide 93 takes a similar conformation in the crystalline state $\left.{ }^{104}\right)$.

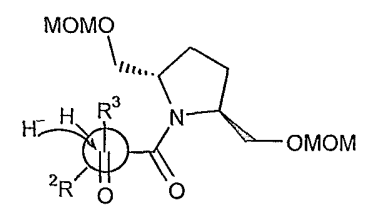

Fig. 2.10 The Felkin-Ahn transition state

However when the reduction with $\mathrm{KBEt}_{3} \mathrm{H}$ was carried out on the system where the pyrrolidine was replaced by an oxazolidinone, the reduction went too far, with the $\beta$ ketoimide being reduced right back to the oxazolidinone (Scheme 2.20), with no measurable presence of any of the desired alcohol. Thus the reagent was too powerful (which would also be the case with $\mathrm{LiAlH}_{4}$ ), which may be a result of the coordination of the $\mathrm{KBEt}_{3} \mathrm{H}$ with the carbonyl of the oxazolidinone, which is not present in the literature example (Scheme 2.19). 


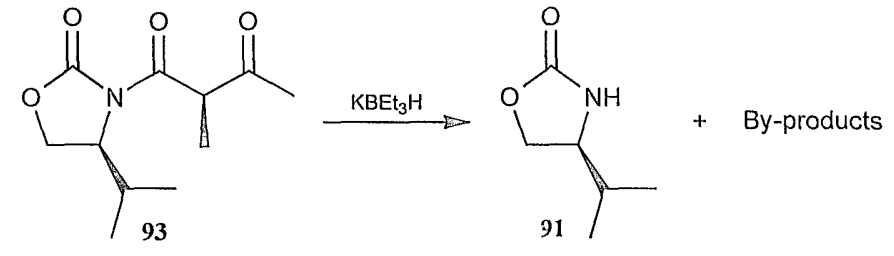

Scheme 2.20

Other reducing agents were therefore considered. In the reductions of various chiral $\alpha$-keto acids and esters, $\alpha$-amino ketones and $\beta$-hydroxyketones, diisobutylaluminium hydride has been employed, but with the resulting products corresponding once again to the erythro isomers. ${ }^{131,124,132}$

Our next efforts concentrated on reductions with $\mathrm{NaBH}_{4}$. This reducing agent generally does not result in diastereoselective reduction, ${ }^{131}$ however both diastereoisomers could be used in our studies, with the incorrect isomer $(2 R, 3 S)$ acting as a 'control' in any feeding experiments. The use of mixed solvent systems $\left(\mathrm{CH}_{2} \mathrm{Cl}_{2}-\mathrm{MeOH}\right.$ or THF-MeOH) have been shown to increase the diastereoselectivity of reductions involving $\mathrm{NaBH}_{4}$, generally in favour of the threo isomer, ${ }^{124,133}$ which can be explained by the formation of methoxyborohydride which might be more selective in the required sense than $\mathrm{NaBH}_{4} \cdot{ }^{134,135}$ Thus, the conditions for the reduction of the $\beta$-keto imide 93 were investigated in order to increase the yields of the reaction. A mild reduction of 93 with $\mathrm{NaBH}_{4}$ in a solvent mixture of $\mathrm{CH}_{2} \mathrm{Cl}_{2}-\mathrm{MeOH}(1: 1)$ at $0{ }^{\circ} \mathrm{C}$ was employed which resulted in a mixture of the diastereoisomeric products 99 and $\mathbf{1 0 0}$ in an overall yield of $80 \%$. The NMR of the crude mixture indicated the presence of the two diastereoisomers in a ratio of $1: 2$, 
however the assignment of the major isomer at that time was not possible. Attempts were made to separate the diastereoisomers but under all the solvent systems tried, the TLC still only indicated a single spot. Ester derivatives of compounds 99 and 100 were synthesised, which it was hoped might be separable. The best general method for the preparation of esters is considered to be via the reaction of alcohols and acyl halides. ${ }^{136}$ In this study, this reaction was attempted using 3,5-dinitrobenzoyl chloride, as seen in Scheme 2.21. One of the reasons this reagent was chosen, was that the product formed would be easily detected by TLC because of its UV activity, which would assist in the separation of the products. The reaction was then carried<smiles>C=C(C)C(=O)N1C(=O)OC[C@H]1C(C)C</smiles><smiles>O=C(Cl)c1cc([N+](=O)[O-])cc([N+](=O)[O-])c1</smiles><smiles>C=C(C(=O)N1C(=O)OC[C@H]1C(C)=O)C(C)C(=O)O[C@@H](C)C(C)C(=O)N1C(=O)OC[C@H]1C(C)C</smiles>

Scheme 2.21 
out on the mixture of alcohols 99 and 100 with 3,5-dinitrobenzoyl chloride (2 equiv.), in the presence of pyridine (2.2 equiv.) at $0{ }^{\circ} \mathrm{C}$. The reaction was followed by TLC and when all the starting material had disappeared, the reaction mixture was quenched with dilute $\mathrm{HCl}$, and washed with further $\mathrm{HCl}$ to remove any traces of pyridine. The recovered crude solid product gave an NMR that showed the presence of very little starting material. The NMR showed a separation of the doublets corresponding to the isopropyl methyls of the chiral auxiliary (Fig. 2.11). These signals, which appeared as a pair of doublets in the NMR of the mixture of derivatives ( $\delta 0.90$ and 0.94 ; for both diastereoisomers), were now separated into two distinct pairs of doublets (at $\delta 0.74$ and 0.82 and $\delta 0.91$ and 0.93 ) corresponding to the two diastereoisomers 104 and $\mathbf{1 0 5}$. The dinitrophenyl group has a great effect on the chemical shift of these hydrogens, due to the deshielding effect (note particularly the shift of the hydrogen attached to the ester carbon which has shifted downfield by ca. 2 ppm from $\delta 3.8$ to 5.6), suggesting the derivatives may now be sufficiently 'chemically different' to separate.

The TLC system used (ethyl acetate-hexane, 1:1) to follow the reactions, however still only showed the presence of a single spot for the reaction mixture. Thus investigations were undertaken to find a solvent system in which the two diastereoisomers would separate. This was eventually found with ethyl acetatetoluene $(1: 3)$ under which conditions, two spots by TLC could be identified $\left(R_{\mathrm{f}} 0.63\right.$ and 0.54). 


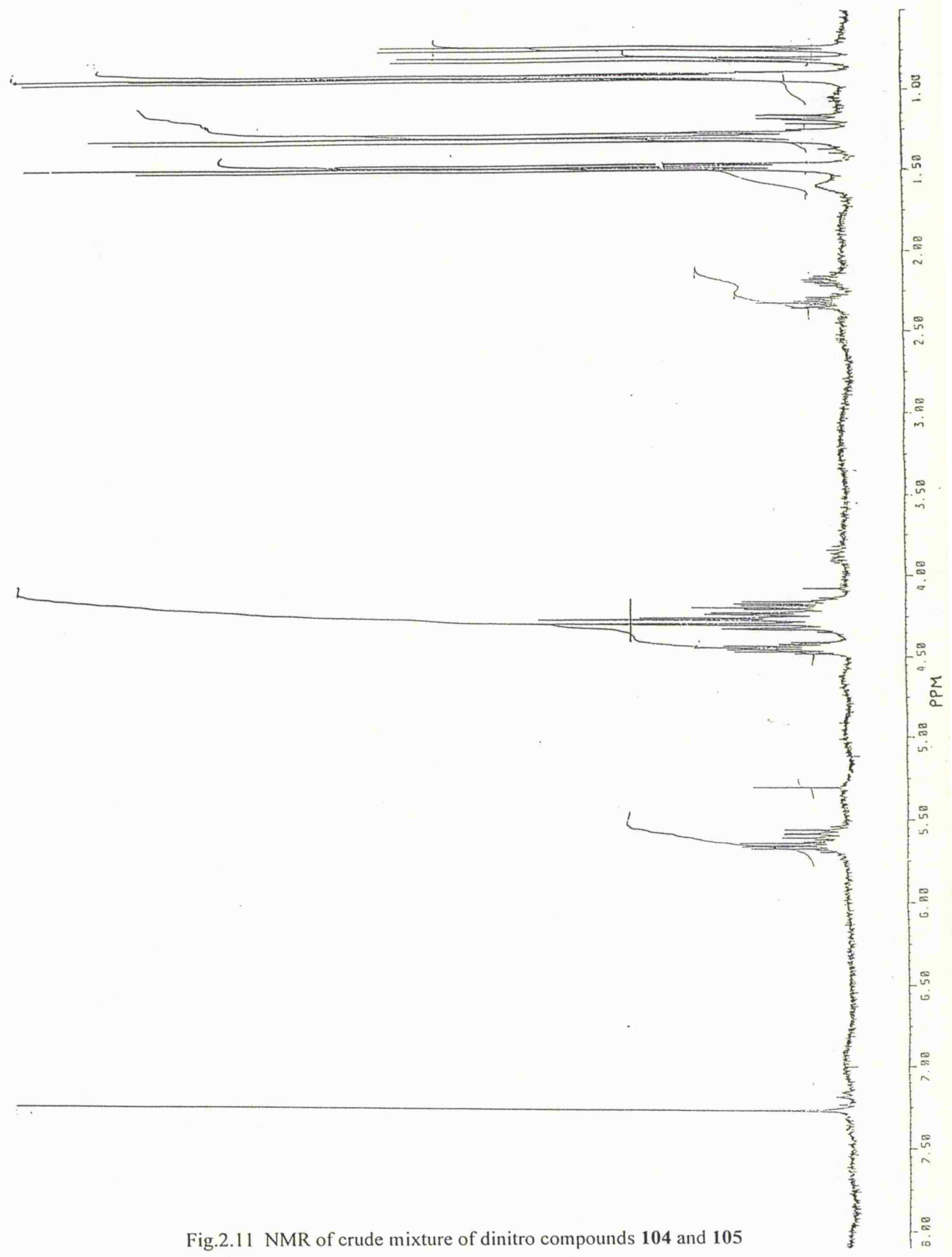


The successful separation of the two diastereoisomers was carried out by flash column chromatography with the solvent system of ethyl acetate-toluene (1:99). However, at this stage the stereoisomerism of each of the products was still unknown and so crystals were collected which were satisfactory for X-ray crystallography. Slow recrystallisation from a variety of solvents was carried out, with the solvent system of THF-toluene (1:1, with slow evaporation) giving white-pale yellow large crystals of the fasting rumning spot. The X-ray crystal structure of the compound corresponding to the fastest running spot by TLC can be seen in Fig. 2.12 (see appendix $\mathrm{A}$ for bond lengths and angles) ${ }^{\dagger}$ which is the $2 R, 3 S$ isomer, compound $\mathbf{1 0 5}$, and thus the bottom spot is the $2 R, 3 R$ isomer 104 , the enantiomer that is required for feeding experiments.

With the successful separation of the two diastereoisomers, the final steps in the synthesis were investigated on compound 105 . These involved the hydrolysis of the chiral auxiliary, along with the dinitrobenzoate ester group, to give the free $\alpha$ methyl- $\beta$-hydroxy acid, which then could be converted to the NAC thioester derivative. The hydrolysis of the two different ester derivatives could either be carried out separately (i.e. one first, then the other, perhaps under different conditions) or simultaneously. It was considered that under hydrolytic conditions, the dinitrobenzoate ester would be easily cleaved due to the nitro groups being electron withdrawing and thus making it more susceptible to hydrolysis and mild hydrolysis conditions were sought that would initially only cleave the dinitro-

\footnotetext{
${ }^{\dagger}$ Thanks to Dr John Fawcett, Chemistry Department, University of Leicester, solving the structure.
} 


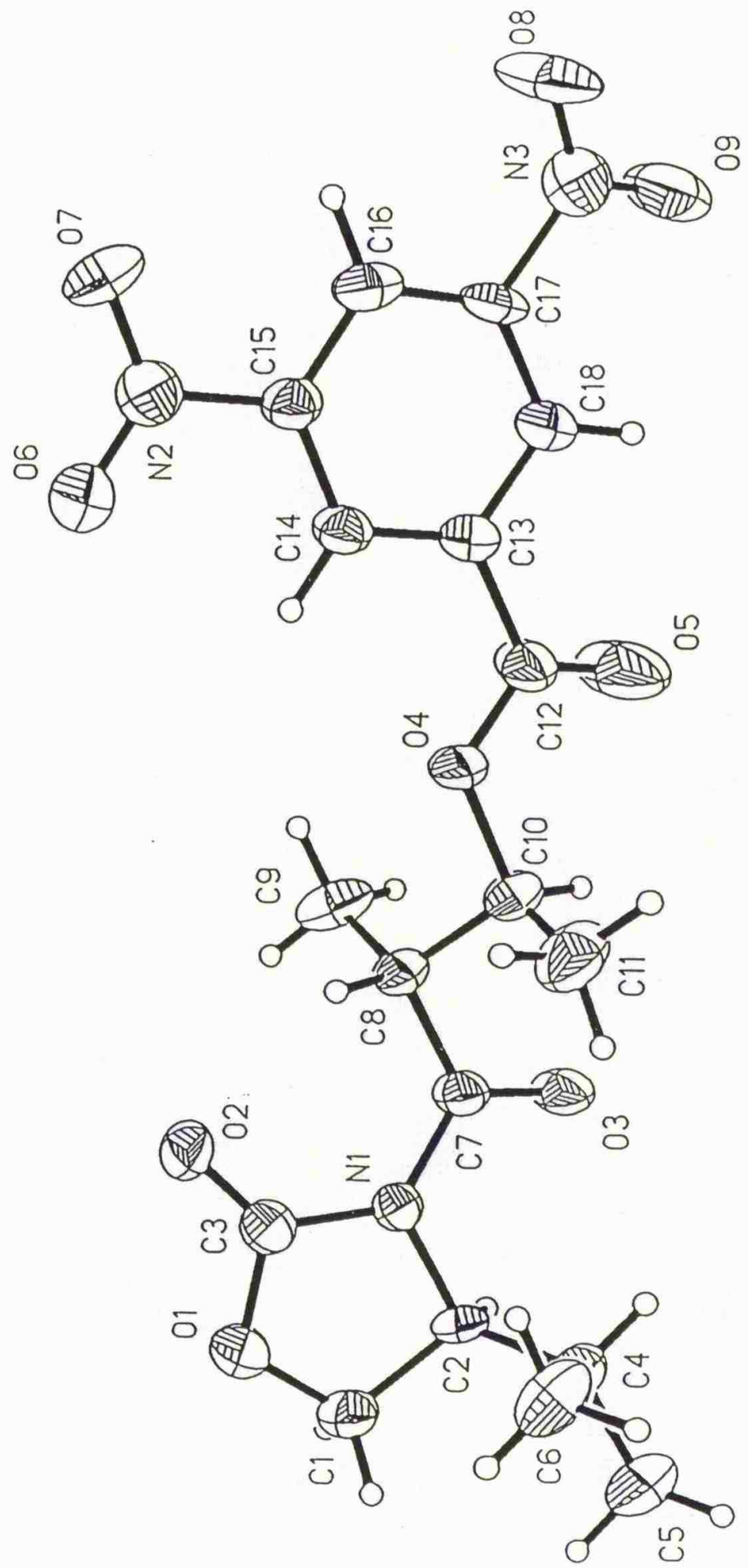

Fig.2.12 X-ray crystal structure of 105 
benzoate ester. This was attempted with sodium hydroxide solution in dioxane $\mathrm{e}^{137}$ on a number of occasions (heat/reflux, 15-30 min), however only the starting material was recovered. The conformation and steric hindrance to attack on either face of the dinitrobenzoate ester carbonyl group (see Fig. 2.12) make it less likely to be cleaved and so a hydrolysis of both the ester and imide together was attempted.

Evans et al. have reported their efforts to cleave the chiral auxiliary from the $\mathrm{N}$-acyl derivatives. ${ }^{138}$ Lithium hydroperoxide generated in situ proved to be more selective than lithium hydroxide in the deacylation of the oxazolidinone, displaying a much greater regioselectivity for exocyclic carbonyl cleavage and resulting in the production of the free acid and the recovery of the chiral auxiliary. This is probably due to the $\mathrm{HOO}^{-}$being more nucleophilic than $\mathrm{OH}^{-}$in aqueous solutions due to its smaller solvation energy.

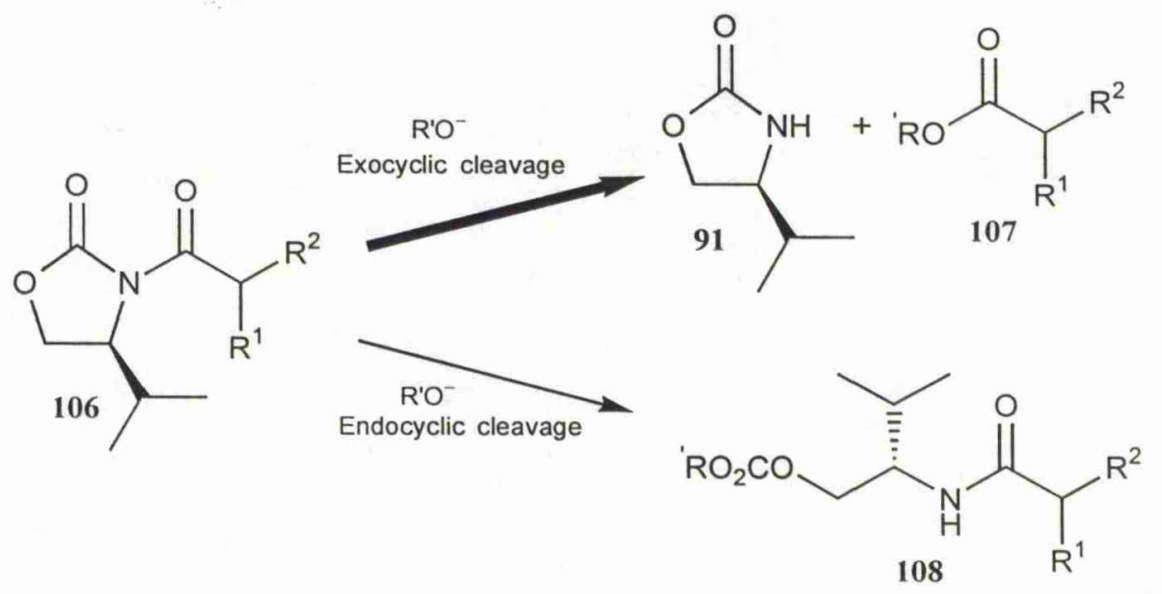

Scheme 2.22 
In our study, the hydrolytic cleavage of the chiral auxiliary was attempted in conjunction with that of the dinitrobenzoate ester functionality. Lithium hydroperoxide was generated in situ and added to a solution of the control diastereoisomer 105 in THF- $\mathrm{H}_{2} \mathrm{O}(4: 1)$ under nitrogen, which was stirred for $2 \mathrm{~h}$ at 0 ${ }^{\circ} \mathrm{C}$. After work up, dichloromethane extraction followed to remove the cleaved chiral auxiliary. The aqueous layer was then buffered with dilute acid to enable the product acids (109 and $\mathbb{1 1 0}$ ) to be extracted into ethyl acetate (Scheme 2.23).

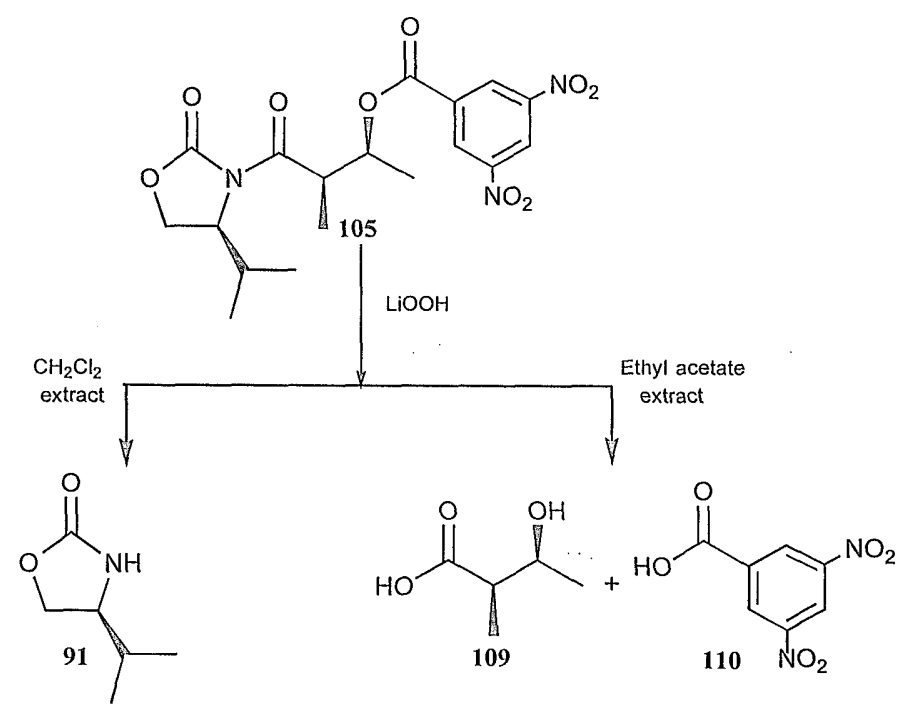

Scheme 2.23 
The NMR of the crude ethyl acetate extract mixture was then examined in order to determine the presence of the two acids. The $3 \mathrm{H}$ multiplet at $c a . \delta 9.2$ was assigned to the $3 \mathrm{H}$ of the benzoate group, which was in a similar position to the starting material. The two $3 \mathrm{H}$ doublets at $c a$. $\delta 1.25$ corresponding to the $\alpha$ and $\beta$ methyl groups, and the $1 \mathrm{H}$ multiplet at $\delta 2.45$ corresponding to $\mathrm{H}_{\alpha}$ were as expected (the latter having moved upfield slightly because the oxazolidinone group had been removed). Now, there was the possibility that this procedure had removed the oxazolidinone group only, with the dinitrobenzoate group being left intact. If this was so, the signal corresponding to $\mathrm{H}_{\beta}$ would have remained downfield (at $\delta$ 5.6). However, this is not the case, with the $1 \mathrm{H}$ multiplet appearing at $c a . \delta 3.80$, thus indicating the dinitrobenzoate group had indeed been hydrolysed, and confirming the presence of the two acids $\mathbf{1 0 9}$ and $\mathbf{1 1 0}$ in the crude mixture. However, these products were difficult to detect by TLC, which made separation difficult. Since the final step in the synthesis requires the formation of a thioester derivative, it was anticipated that the corresponding two products formed would be separable. Therefore the crude ethyl acetate extract, containing the two free acids (109 and 110), was converted into the corresponding mixture of NAC thioesters.

For the final stage of this synthesis, $N$-acetylcysteamine was first prepared by monohydrolysis of $N, S$-diacetylcysteamine in a degassed solution of water with 


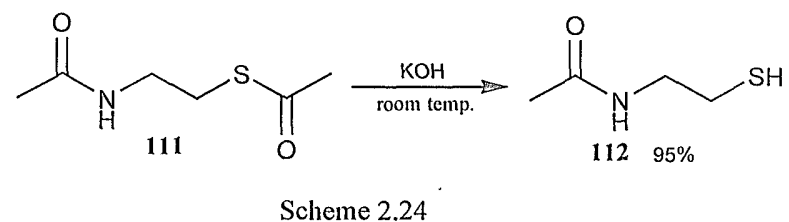

potassium hydroxide in almost quantitative yield (Scheme 2.24). ${ }^{139}$ The $N$-acetylcysteamine $\mathbf{1 1 2}$ that is produced rapidly decomposes and so was used immediately in the next step.

The mixture of acids (109 and 110) underwent reaction as shown in Scheme 2.25. ${ }^{140,141}$ The mechanism for this reaction can be seen in Scheme 2.26, in which the acids are activated by DCC with DMAP present as a catalyst. Work up of the reaction involved filtering the solution through Celite, which was washed thoroughly with ethyl acetate (to remove any traces of residual dicyclohexylurea). The filtrate was evaporated to give a crude brown red oil red oil/crystals that contained a variety of products as shown by TLC (ethyl acetate), which included at least one UV active material and other spots stained by phosphomolybdic acid. To provide us with the information we needed as to where to expect the NAC thioester product $\mathbf{1 1 3}$ to run on TLC in such a solvent system, the racemic product 118 was synthesised from ethyl methylacetoacetate, which could also be used for comparison in determining peaks in the NMR etc. 

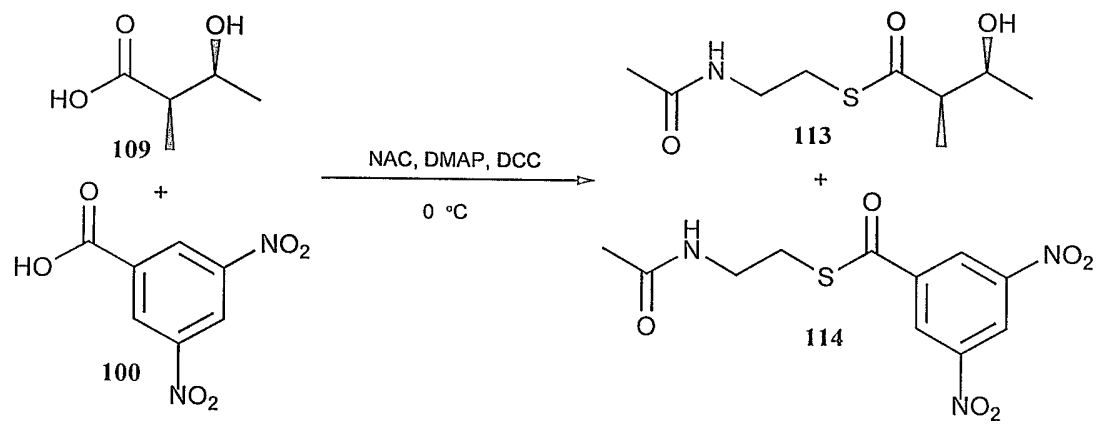

Scheme 2.25

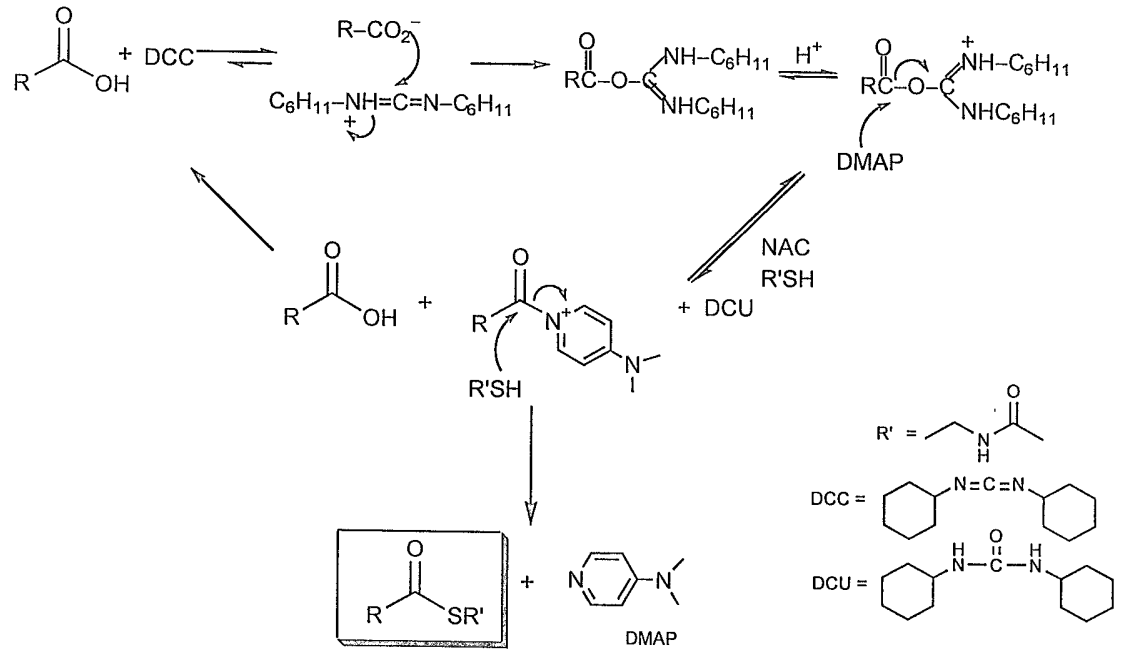

Scheme 2.26 
Thus, ethyl methylacetoacetate 115 was reduced with sodium borohydride at $0{ }^{\circ} \mathrm{C}$ to give ethyl-2-methyl-3-hydroxybutanoate $\mathbb{1 1 6}$ as a cream oil in $80 \%$ yield (Scheme 2.27). This ester was then further hydrolysed with potassium hydroxide to give the free acid 117 (pale yellow oil) in 63\% yield, which was identified by the NMR of the ethyl acetate evaporated extract. This extract was then reacted in the manner described above for the conversion of the acid into the NAC thioester. The TLC of the crude product (ethyl acetate) showed three major spots (stained with phosphomolybdic acid) which when separated by flash column chromatography (acetone-toluene, 1:2) were identified as residual dicyclohexylurea $\left(R_{\mathrm{f}} 0.73\right)$, NAC thioester $118\left(R_{\mathrm{f}} 0.19\right)$ and unreacted $N$-acetylcysteamine $\left(R_{\mathrm{f}} 0.32\right)$. The product was purified in $33 \%$ yield and was identified by ${ }^{1} \mathrm{H}$ NMR.

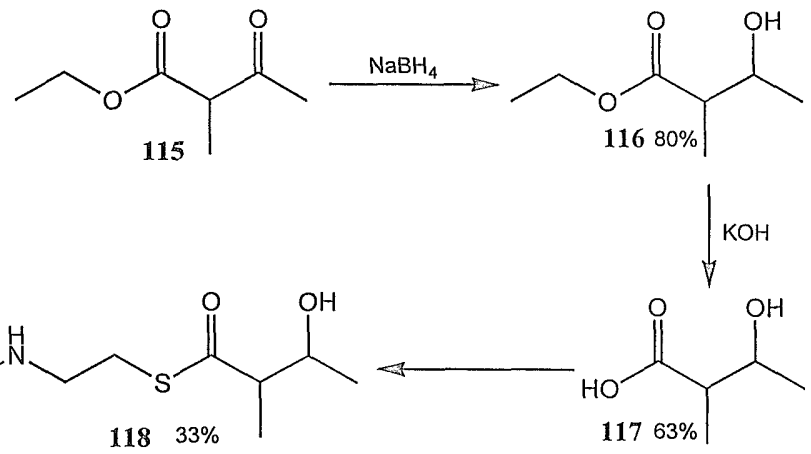

Scheme 2.27 
Now the racemic thioester had been prepared and the $R_{\mathrm{f}}$ value of the products was known, the crude mixture of the control product could be purified. Exactly the same column conditions were utilised, this time with the separation of four major spots; three the same $R_{\mathrm{p}} \mathrm{s}$ as previously and the fourth $\mathrm{UV}$ active spot of the dinitrobenzoate thioester $114\left(R_{\mathrm{f}} 0.48\right) .(2 R, 3 S)$-3-Hydroxy-2-methylbutanoyl $N$-acetylcysteamine thioester 113 was isolated in ca. $40 \%$ yield.<smiles>CC(=O)NCCSC(=O)C(C)=O</smiles> 


\subsubsection{2 $(\mathbb{R})$-Valine route and incorporation of labels}

As discussed previously, the target compound actually has the $(2 S, 3 S)$ configuration, and so this series of reactions then had to be carried out starting with the opposite enantiomer i.e. $(R)$-valine $\mathbf{1 1 9}$, with the incorporation of the deuterium labels at the appropriate stages. Thus, the reduction of $(R)$-valine with $\mathrm{LiBH}_{4}$ and TMSCl in THF was once again carried out in good yields $(78 \%)$ to give $(R)$-valinol 120 , which was then reacted with diethyl carbonate to produce the (4R)-4-isopropyloxazolidin-2-one 121 as white crystals in $83 \%$ yield (Scheme 2.28 ).

The acetyl oxazolidinone 122 was then prepared by addition of BuLi to a solution of 121 in THF to form the enolate, followed by reaction with acetyl chloride, which went in good yield $(76 \%)$ (Scheme 2.29$)$. The next reaction involved the methylation of this product to form the $N$-propionyloxazolidinone 123 with the incorporation of the deuterium labels. Due to the problems encountered in this step on the $(S)$-valine system, effort was taken to ensure the greatest possible yields as the<smiles>CC(C)[C@@H](CO)NC(=O)[C@@H](N)C(C)C</smiles>

Scheme 2.28 


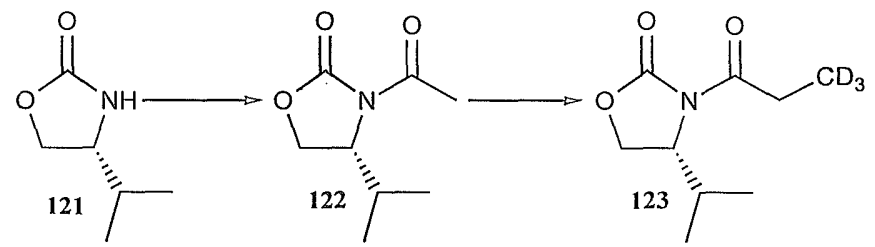

Scheme 2.29

deuterium labelled iodomethane was expensive. Once again, the formation and slow warming of the enolate in the presence of deuteriated methyl iodide was important for the success of this reaction, with $\mathbf{1 2 3}$ being produced in $45 \%$ yield.

The enolate of compound $\mathbf{1 2 3}$ was next formed with LDA under similar conditions to those described previously and reacted with acetyl chloride to produce the $\beta$-keto imide 124 in $47 \%$ yield (Scheme 2.30 ). This reaction was once again highly diastereoselective with the pure single diastereoisomer being isolated after flash column chromatography as white crystals.

The next step involved the reduction of compound 124 with the simultaneous incorporation of the final deuterium label. After our experience with the unlabelled system, it was decided to carry out this reduction using the readily available sodium borodeuteride. The reduction was carried out under similar conditions to the 

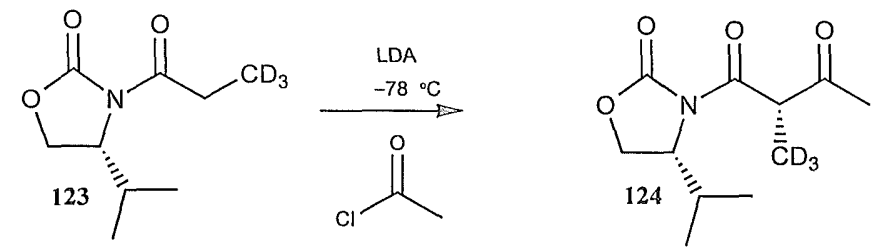

Scheme 2.30

unlabelled $\beta$-keto imide, with 124 dissolved in a solvent mixture of $\mathrm{MeOH}-\mathrm{CH}_{2} \mathrm{Cl}_{2}$ $(50: 50, \mathrm{v} / \mathrm{v})$ at $0{ }^{\circ} \mathrm{C}$. To this, $\mathrm{NaBD}_{4}$ was added and the mixture stirred for $0.5 \mathrm{~h}$ at 0 ${ }^{\circ} \mathrm{C}$ (followed by TLC). The NMR of the crude mixture showed the presence of the two diastereoisomers once again, with the signals for the terminal methyl groups appearing as two separated broad singlets for each diastereoisomer at $c a . \delta 1.2$ (in a ratio of $1: 2$ ). The crude material was purified by flash column chromatography to give the mixture of the two alcohols $(\mathbf{1 2 5}$ and $\mathbf{1 2 6})$ in an overall yield of $c a .50 \%$ (Scheme 2.31). This mixture was then reacted with the 3,5-dinitrobenzoyl chloride, as previously described to give a crude mixture, whose NMR showed only the presence of the derivatised compounds $\mathbf{1 2 7}$ and 128 again in $c a .50 \%$ yield. The crude material was then once again subjected to flash column chromatography in a solvent system (ethyl acetate-toluene, 1:99) that previously enabled the separation of the two diastereomeric products. When the products were separated it turned out that the recovered ratio of products was not as favourable as first envisaged, with $500 \mathrm{mg}$ of the $2 S, 3 R$ product 128 (control diastereoisomer) and only $82 \mathrm{mg}$ of the $2 S, 3 S$ product 127 (desired diastereoisomer). 


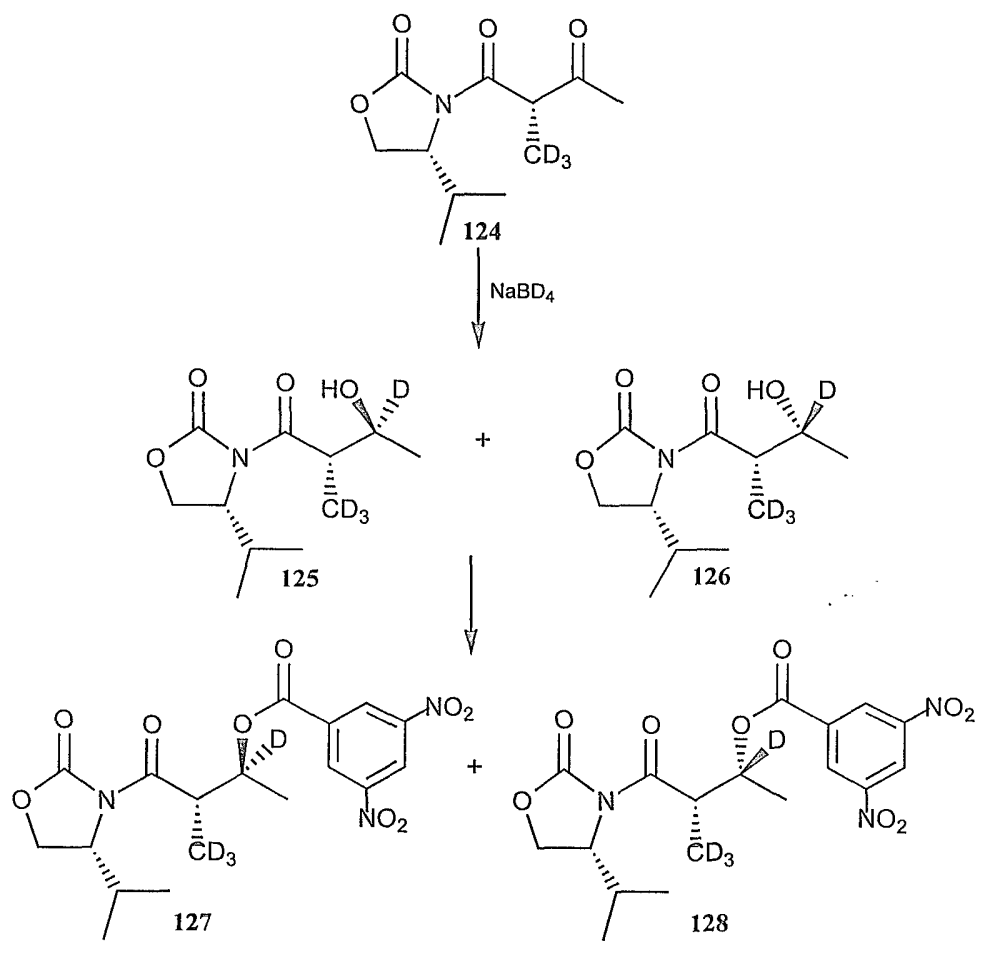

Scheme 2.31

Nevertheless, the final steps of the synthesis were then carried out (Scheme 2.32).

The desired diastereoisomer $\mathbf{1 2 7}$ first underwent the hydrolysis to remove both the chiral auxiliary and dinitrobenzoate group in one step. This was carried out under the same conditions as previously, with the hydrolysis being carried out by the LiOOH 
that was prepared in situ. The NMR of the crude ethyl acetate extract showed the expected peaks of the two free acids, with the broad $3 \mathrm{H}$ singlet corresponding to the terminal $\mathrm{CH}_{3}$ group of 129 and the $1 \mathrm{H}$ broad singlet of $\mathrm{H}_{\alpha}$ on the carbon adjacent to the $\mathrm{CD}_{3}$ group, and the multiplet corresponding to the aromatic $3 \mathrm{H}$ of the benzoate group of 130. This mixture was then taken on to form the NAC thioester by reacting with $\mathrm{N}$-acetylcysteamine, DMAP and DCC, the crude product of which showed a number of products present by TLC. Under similar conditions to previously, this mixture was purified to give $8 \mathrm{mg}$ of the pure desired NAC thioester 51 as a clear oil (ca. $30 \%$ yield from the free acid). The control product was prepared in a similar way, with the hydrolysis of the ester imide derivative 128 followed by the convenient conversion of the free acids (131 and 132) to the corresponding NAC thioesters; from the resulting mixture the control NAC thioester 133 was isolated as an oil $(16 \mathrm{mg}, c a$. $40 \%$ yield from the free acid).

Thus, the chemical synthesis was complete. Although this may not be the most elegant synthesis of the desired compounds, the main aim was to produce sufficient amounts of material for the biological experiments via a method that would fulfil the original criteria. This method turned out to be rather more unwieldy than first anticipated, with lower yields being gained than expected from enantiomeric compounds. The sequence of reactions was repeated in the hope of increasing the 

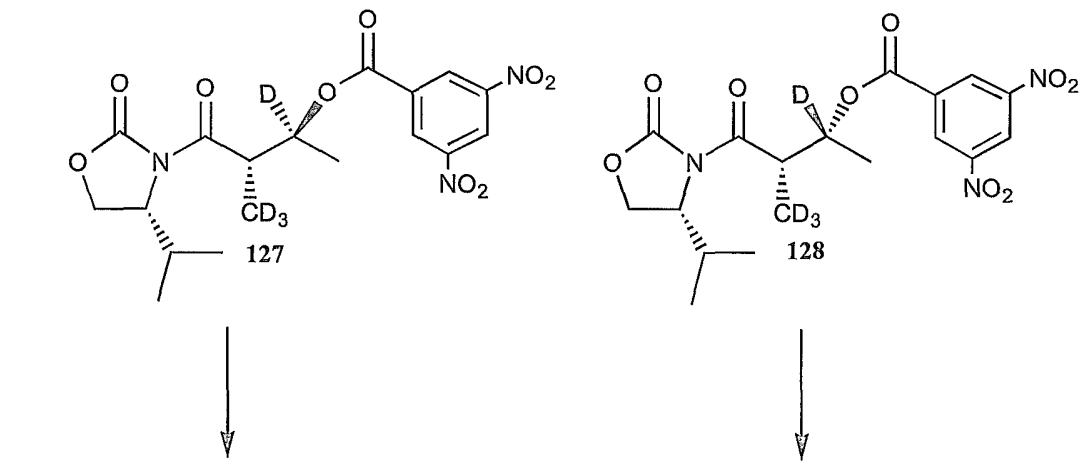<smiles>O=C(O[18O])c1cc([N+](=O)[O-])cc([N+](=O)[O-])c1</smiles>

1<smiles>C[C@H](C(=O)SCCNC(=O)C1CC2CCC1C2)C(C)(O)O</smiles>

TARGET COMPOUND $(2 R, 3 R) 51$<smiles>O=C(O)c1cc([N+](=O)[O-])cc([N+](=O)[O-])c1</smiles><smiles>[AlH][V]</smiles><smiles></smiles>

CONTROL ISOMER $(2 R, 3 S) 133$

Scheme 2.32 
yield of the final $\mathrm{N}$-acetylcysteamine thioester, but similar yields were gained in all the reaction steps. Alternative methods were not investigated at this stage as sufficient quantities of the labelled diketides as the NAC thioester derivatives (51 and 133) were produced for the biological experiments which will be described in the next chapter. 


\section{Chlowpter 3}

\section{Biologicall Studies}

\subsection{Introduction}

Many polyketides and a variety of other secondary metabolites possess important antibacterial, antifungal and antiparasitic properties. Whilst polyketides are produced by a variety of organisms, many are produced by Gram positive soil bacteria, such as the streptomycetes. The ability of microorganisms to produce compounds that exhibit such antimicrobial properties is possibly linked to environmental conditions concerning competition etc. that the organisms encounter. Metabolite production appears to be intricately involved with the complex life-cycle of these advanced bacteria, and is the subject of much research. ${ }^{142}$ Our initial biological studies in this study involved the determination of the optimum conditions for the production of amphotericin B by $S$. nodosus. The conditions for the growth of this microorganism had not previously been investigated in our group and thus a great deal of time and effort was invested in determining the optimum protocols. 


\subsection{Growth and production}

\subsubsection{General}

The growth and fermentation of microorganisms can occur on a range of substrates, enabling the production of a wide variety of products. Microorganisms have a number of prerequisites for growth, and in order for the metabolites to be produced, a number of precise cultural conditions need to be met. For bacteria, the requirements for growth include the presence of sources of energy, nitrogen, phosphate, sulfate and various trace elements, and sometimes also vitamins, all of which need to be present in specific ratios. The temperature, $\mathrm{pH}$ and oxygen levels are also critical for maximum growth of the bacteria and production of the microbial metabolite.

The growth of bacteria proceeds through a number of specific stages, as can be seen represented in Fig. 3.1. The lag phase is a period of adaptation following the inoculation of an appropriate medium. After a period of time that is variable for different microorganisms, the number of cells begins to increase and does so at a constant rate (the log phase). As the levels of certain nutrients begin to diminish or toxic substrates start to accumulate, the growth of the microorganism enters the stationary phase. After some time, cell lysis begins to occur (the death phase) and the number of viable cells starts to decrease. ${ }^{143,144}$ Both the kinetics and metabolism of the various culture phases are different, with microbial metabolites being produced during the log and stationary phases. Primary metabolic production is most active during the log phase, ${ }^{145}$ with most secondary metabolites being produced towards the 
end of the $\log$ phase and also towards the onset of the stationary phase when the growth of the microorganism is slow and the cells are senescent. In this way, amphotericin B is unusual as it is produced by $S$. nodosus early in the log phase.

As mentioned previously, there is a fine dividing line between the primary and secondary metabolites, with such categories being convenient, but sometimes not entirely accurate. ${ }^{145,146}$ The metabolic routes to produce the primary microbial metabolites are common to a wide

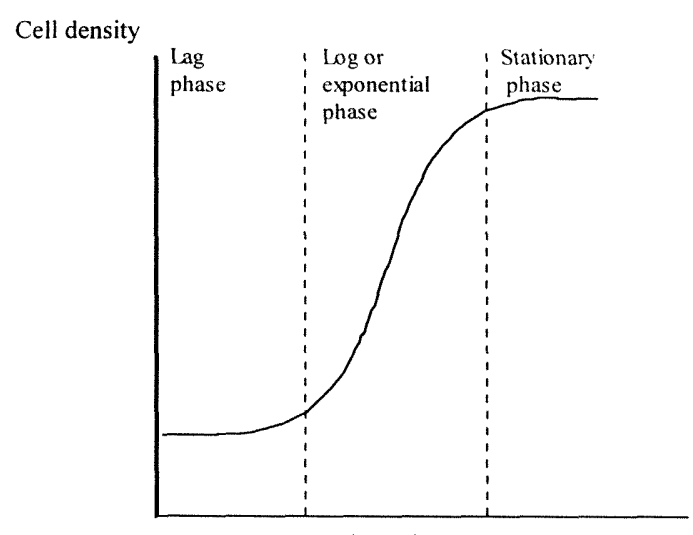

Time after inoculation

Fig. 3.1 Bacterial growth stages

variety of microorganisms, resulting in such products as amino acids, proteins, nucleotides, etc. The production of each individual secondary metabolite, on the other hand, only occurs in very few microbial taxa. Thus even though the taxonomic distribution of the secondary metabolites is far less than the primary metabolites, the possible range compounds produced is immense. Investigations into the onset of, 
and influential effects upon (the induction or repression of), the production of secondary metabolites have been undertaken. It has been shown that a certain mixture of components may result in the good growth of viable cells, but may not produce high levels of metabolite. It has been found that levels of carbon, nitrogen and phosphate that promote and support high growth rates, tend to support poor secondary metabolism. Thus, such repressing nutrients should be kept in mind when ascertaining the medium to be used in the fermentation process. However, varying the levels of nutrients in the media may have a beneficial effect, i.e. nitrogen starvation can stimulate the production of some secondary metabolites. ${ }^{147}$

The fermentation process is summarised in Fig. 3.2. The first stage for any fermentation process involves the formulation of the required medium. When designing the liquid medium there are various criteria that need to be considered (the concentrations of the carbon, nitrogen and phosphate sources, along with the various other substrates and nutrients required, bearing in mind any potential limiting nutrients) in order to produce the optimum medium for a certain metabolite. A number of media recipes will suffice any one microorganism, however only the one will be chosen that achieves the maximum growth and microbial metabolite production.

Once the medium has been formulated, a sufficient inoculum has to be developed. From the pure culture stock, which might be stored as slopes or as vials of spore suspension, an aliquot has to be added to the vegetative medium (the primary seed), a 


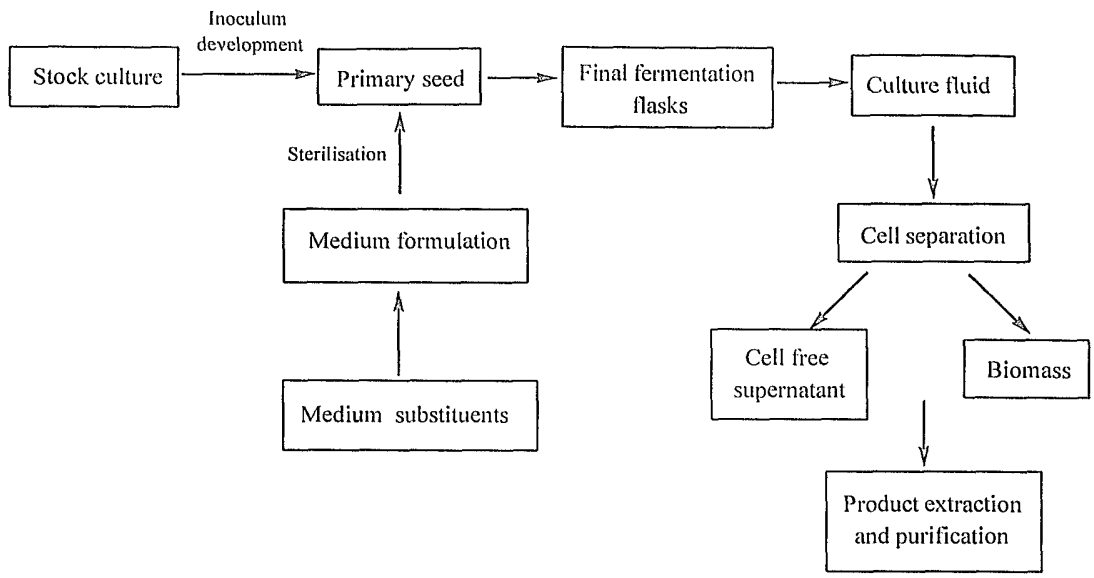

Fig. 3.2 General procedure of the fermentation process

process that might have to be repeated in order to get sufficient growth of the microorganism. The primary seed is generally composed of a nutritionally rich medium which is allowed a few of days growth in order for the spore production to get under way. This is then used to inoculate the final media, which allows for a more reproducible inoculum as it results in a more even concentration of spores being added to the production medium (rather than using them directly off a slope). The exact quantities of inoculum and the ratios of volume of liquid media to volume of the shake flask is very important, particularly where aeration is required for maximal growth of the microorganism, as is the temperature and speed at which the fermentation flasks are shaken. 


\subsubsection{Amphotericin B}

The growth and production of the numerous antifungal polyene antibiotics that have been isolated has been studied widely. ${ }^{147}$ There are many examples of complex mixtures of antifungal substances being obtained from microorganisms that produce the polyenes. Both polyene and non-polyene antibiotics may be co-produced by the same microorganism, however in the case with $S$. nodosus, there is the coproduction of two closely related polyenes; a heptaene (amphotericin B) and a tetraene (amphotericin A) (Fig. 3.3). Most polyene antibiotics grow under submerged aerobic conditions at temperatures ranging between 26 to $34{ }^{\circ} \mathrm{C}$ in a nutrient broth containing one or more nitrogen sources, a carbon-containing metabolisable energy source and inorganic salts. ${ }^{146}$ Energy sources used include carbohydrates, sugars, alcohols and animal and vegetable oils. Fermentation is usually stopped anywhere between 90

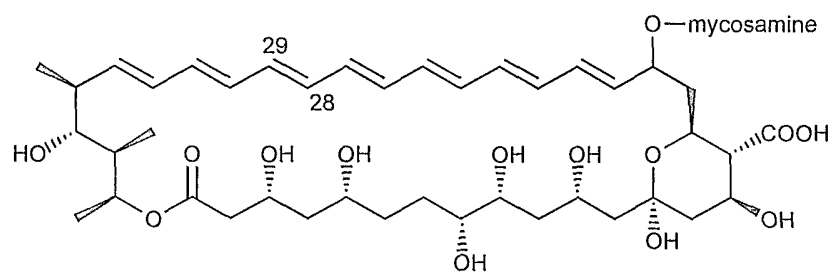

Fig. 3.3 Amphotericin B and amphotericin A (C28-C29 dihydro-analogue)

and $168 \mathrm{~h}$ after inoculation. A variety of extraction methods may then be undertaken in order to recover the produced antibiotic, which will be discussed in the next section. In order to measure the rate of production for any antibiotic in the 
fermentation flask, an assay must be determined. A general characteristic of polyene antibiotics is their yellow colour which increases in intensity from pale to dark yellow, with the increasing number of double bonds, which may be a general qualitative assay. A more quantitative assay involves the characteristic ultraviolet absorption that is observed due to the chromophores of the unsaturated straightchain, and it is this that is used to classify the different antibiotics into tetraenes, pentaenes, hexaenes and heptaenes. The characteristic UV absorption for amphotericin B can be seen in Fig. 3.4, with the absorption peaks being detected at the wavelength of $346,364,382$ and $405 \mathrm{~nm}$. A similar absorption spectra is produced for amphotericin A, with peaks at 280, 292, 305 and $320 \mathrm{~nm}$. The characteristic UV absorption which arises because of the chromophore can be conveniently used to measure the concentration of the antibiotic in any given solution

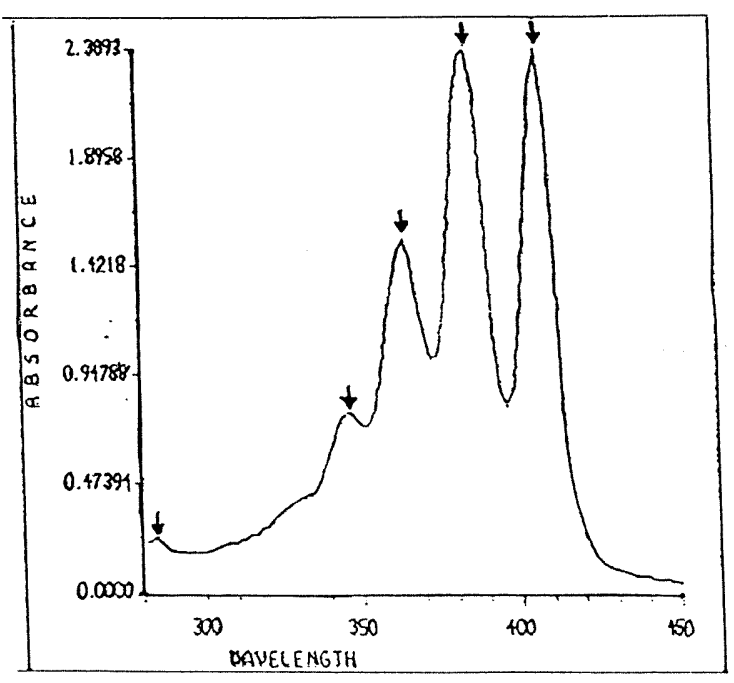

Fig. 3.4 Characteristic UV of amphotericin B 
and thus to establish whole broth titres. Such a spectrophotometric assay utilises the $\varepsilon^{10 \%}{ }_{\mathrm{Icm}}$ value of the pure antibiotic. ${ }^{78}$ For amphotericin $\mathrm{B}$, the antibiotic concentration is calculated on the basis of absorption at $\lambda 405 \mathrm{~nm}$ assuming an $\varepsilon^{1 \%}{ }_{\mathrm{Icm}}$ of 1.740 $\left(10 \mu \mathrm{g} \mathrm{cm}^{-3} \mathrm{MeOH}\right)^{78}$, with the concentration of amphotericin $\mathrm{A}$ being measured in a similar manner at $\lambda 305 \mathrm{~nm}$, assuming an $\varepsilon^{1 \%}{ }_{\text {Icm }}$ of $0.850\left(10 \mu \mathrm{g} \mathrm{cm}^{-3} \mathrm{MeOH}\right)$. The assay of the concentration of metabolite in the fermentation process involved extraction of a sample of the culture broth into DMSO (1 in 10 dilution) by sonicating the solution for $20 \mathrm{~min}$, which then underwent centrifugation to separate the insoluble material. A sample of this extract was then further diluted with methanol and the UV absorption measured. Previously there had been no such investigations in our laboratory and so a great deal of time was invested in establishing the optimum fermentation procedure. Initial studies involved investigating amphotericin B production under a variety of conditions with different media, with a time-course of antibiotic production being measured under the various conditions. The different formulations of the fermentation media resulted in different rates of production for amphotericin $\mathrm{B}$, with the greatest production levels being favoured for the biological experiments.

The main aim of the biological studies was to ascertain the ideal conditions for the feeding of the labelled compounds, as shall be discussed in Chapter 4, for which there would be two different processes. The growth conditions and media were investigated, with the acetate feedings requiring a large amount of amphotericin B to be produced in a concentrated media for the NMR studies. For the feeding of the 
diketide precursors, detection of incorporation involved the use of electrospray mass spectrometry, and one advantage of this technique was that only a small amount of labelled amphotericin B was needed to be extracted. Thus a methodology had to be developed that involved the growth of a small quantity of whole broth of culture medium, which could then be extracted and purified. The method of purification for this approach was semi-preparative HPLC, the exact conditions of which had to be investigated. With both types of production experiments, gaining information about the time-course of the production of the antibiotic was important, as this would enable a decision to be made as to (when to carry out the resuspension, if applicable, and) when to add any precursors. Preferably, the cells would need to have reached a stage where the enzymes responsible for amphotericin production were highly active.

\subsubsection{Whole broth production}

The first consideration in the fermentation process is the formulation of an inoculation procedure and to prepare the glucose-yeast extract primary seed. The final production media would then be considered. Previous biosynthetic studies of amphotericin B have involved the use of a complex production media, which is a media that is chemically undefined. The formula of media used in that instance included 5\% glucose, 3\% pharmamedia (cottonseed flour) and 1\% calcium carbonate in deionised water at $\mathrm{pH} 7.7$, which, after sterilisation, was generally inoculated with $10 \%(\mathrm{v} / \mathrm{v})$ of the primary seed and incubated on a shaker at $28^{\circ} \mathrm{C}$ and $245 \mathrm{rpm}$ for seven to eight days. ${ }^{70,71,72}$ Fermentation media of a more defined nature can also be 
used in which all the inorganic ions are added in specific ratios that are predetermined.

It was during my first placement at SmithKline Beecham Pharmaceuticals that initial investigations into the growth and production of amphotericin was undertaken. The production of amphotericin $\mathrm{A}$ and $\mathrm{B}$ in three different production media compositions were compared. The first was a complex media (CM), which was essentially the same as described above but using a $5 \%$ vegetative inoculum, which was the case for all the media studied. The next was the defined medium (DM), in which the nitrogen and inorganic substituents were added in specific quantities $(5 \%$ $\mathrm{w} / \mathrm{v}$ glucose, $1 \%$ calcium carbonate, $0.1 \%$ L-asparagine, $0.01 \% \mathrm{FeSO}_{4}{ }^{\circ} 7 \mathrm{H}_{2} \mathrm{O}$ and $0.01 \% \mathrm{MnCl}_{2} \circ 4 \mathrm{H}_{2} \mathrm{O}$ at initial pH 7.6). The final medium investigated, FCA, was one in which there were two carbon sources present $(2 \%$ fructose, $6 \%$ collofilm dextrin, $3 \%$ arkasoy and $1 \%$ calcium carbonate at $\mathrm{pH} 7.7$ ). All experiments were carried out in $500 \mathrm{~cm}^{3}$ shake flasks with $60 \mathrm{~cm}^{3}$ of media in each flask. This unusually small volume of broth was critical for adequate aeration of the flasks when on the incubator. The results from these experiments can be seen in Fig. 3.5.

It is obvious that the defined media was not a satisfactory production medium for amphotericin B, and so this media was not used in further investigations. When studying amphotericin production, the levels of both amphotericin A and B need to be considered. As can be seen in Fig 3.6, the ratio of amphotericin A:B can vary 


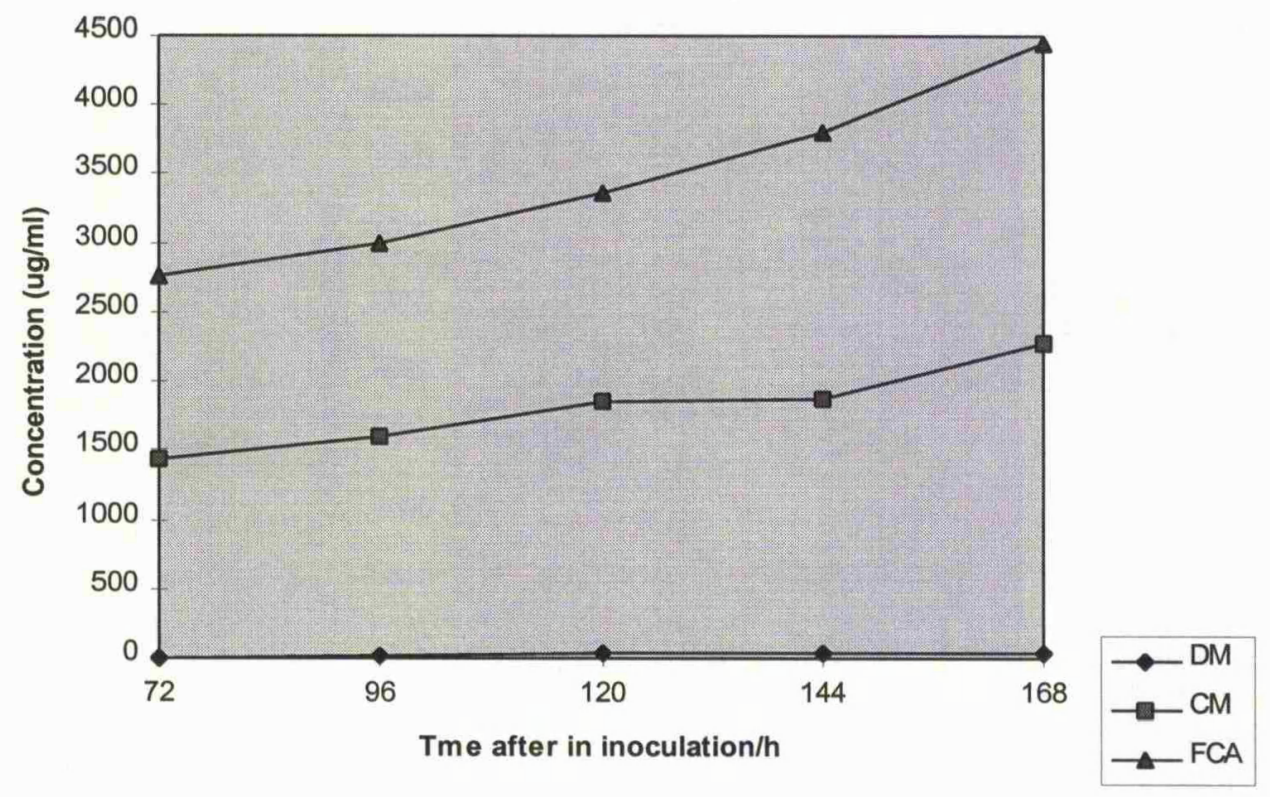

Fig. 3.5 Comparison of amphotericin B production in the 3 different media

greatly, depending upon which production media is used. The concentrations for both $\mathrm{A}$ and $\mathrm{B}$ are almost equal throughout the production time-course for $\mathrm{CM}$ (with the metabolite production having reached an almost constant level after 72) [Fig. 3.6(a)], with equivalent levels of amphotericin A being produced in FCA [Fig. 3.6(b) ]. However, the level of amphotericin B in FCA is almost double of that in $\mathrm{CM}$, with levels continuing to increase upto $168 \mathrm{~h}$ after inoculation. It appears that the production of amphotericin B is affected by the presence of the two carbon sources in the fermentation medium. Studies have shown that these two carbon 
(c)

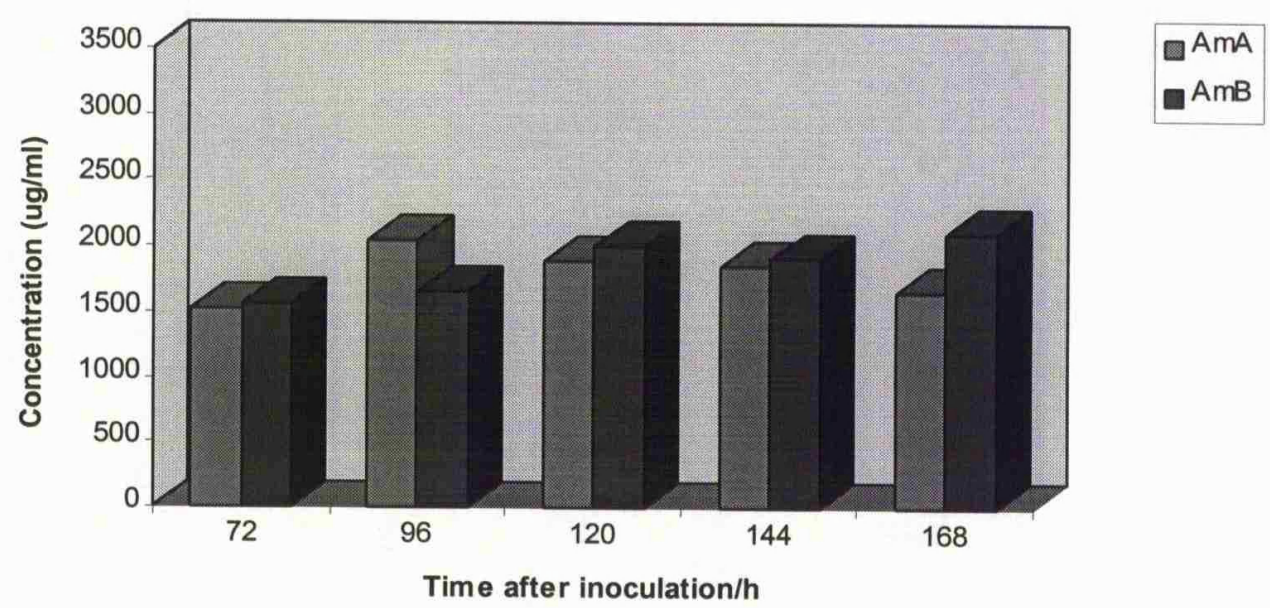

(b)

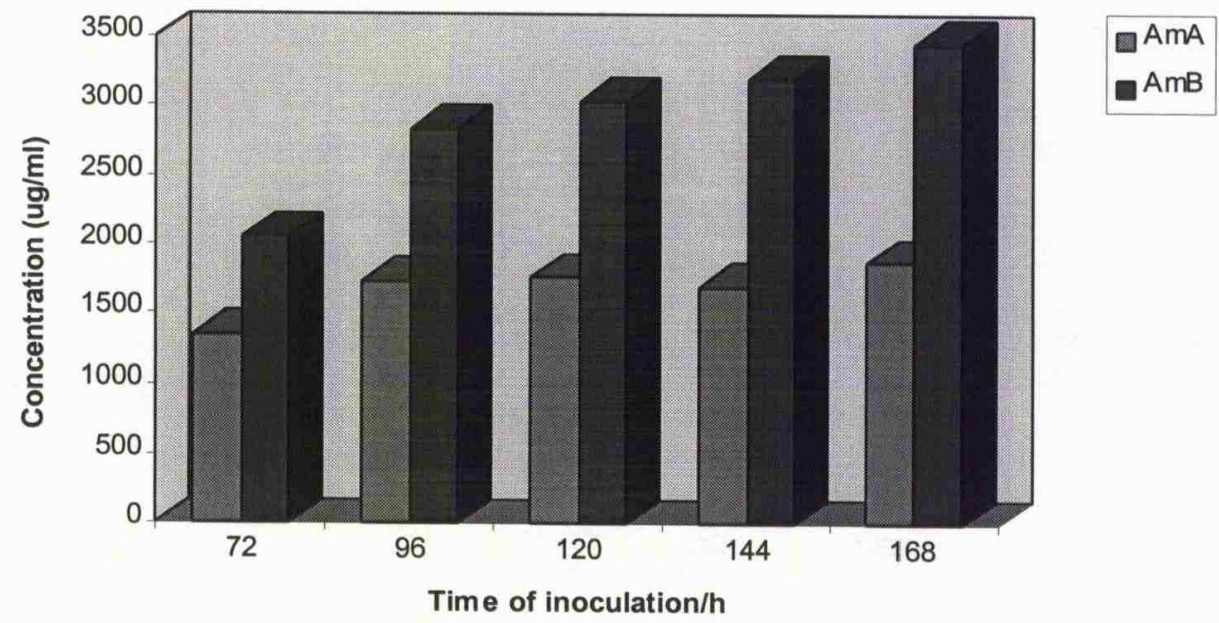

Fig. 3.6 Comparison of amphotericin A and amphotericin B production in (a) CM and (b) FCA media (SmithKline Beecham). 
sources are utilised during different stages of the fermentation process, with the fructose being used during the growth period and collofilm dextrin during the production period..$^{\ddagger}$ It might be considered that the complex media produces less due to the fact that the carbon source is limiting, but when more glucose is added in the media formulation, the concentration of amphotericin B does not increase measurably. Thus, the media of choice was FCA, however on returning to Leicester slightly different results were obtained. As can be seen in Fig. 3.7, the ratio of amphotericin A: amphotericin B was altered, with the complex medium producing twice as much amphotericin A than B, but in much lower overall concentrations. The production in FCA was also altered, however the ratio of amphotericin A:amphotericin B was much the same but with lower concentrations than found previously. Thus, the FCA media was once again the fermentation media of choice, although the reasons for the different levels is still not determined. Conditions used for both fermentation processes were as similar as possible (temperature, flask size, shaker speed etc.), however the production is dependent on so many factors that are not easily reproducible (type of water, the stoppers on the shake flasks, the exact concentration of inoculum) that different results in different environments may not be so surprising, however the general trend is still observed.

Thus, FCA was used as the fermentation media, and in order to get a more detailed idea as to the production of amphotericin, a whole broth time-course was undertaken

${ }^{\ddagger}$ Personal communication, SmithKline Beecham Pharmaceuticals. 
(a)

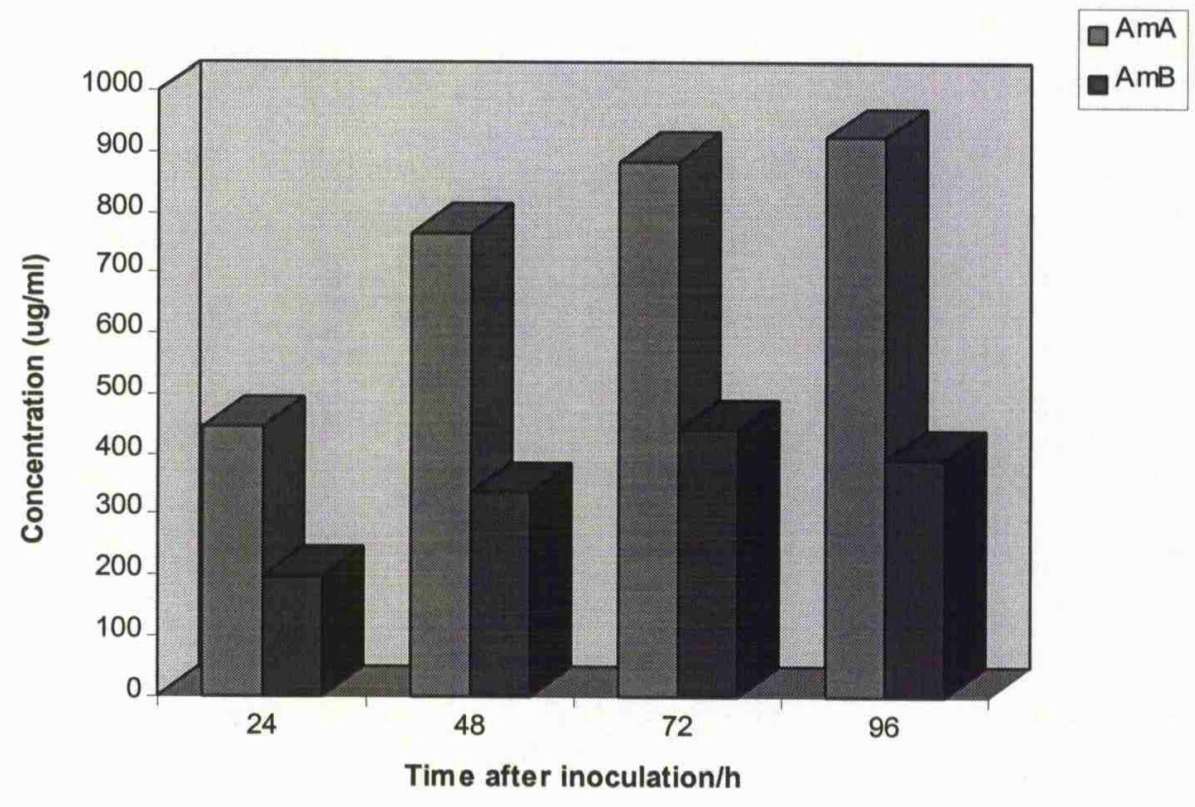

(b)

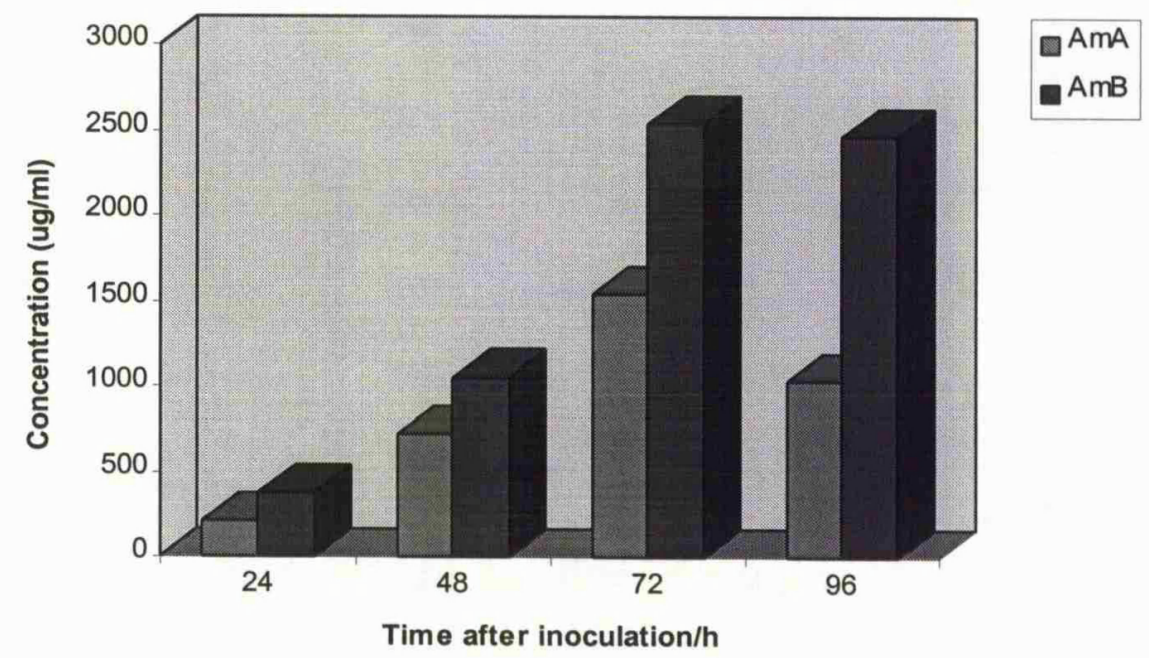

Fig. 3.7 Comparison of amphotericin A and amphotericin B production in (a) CM and (b) FCA media (Leicester) 


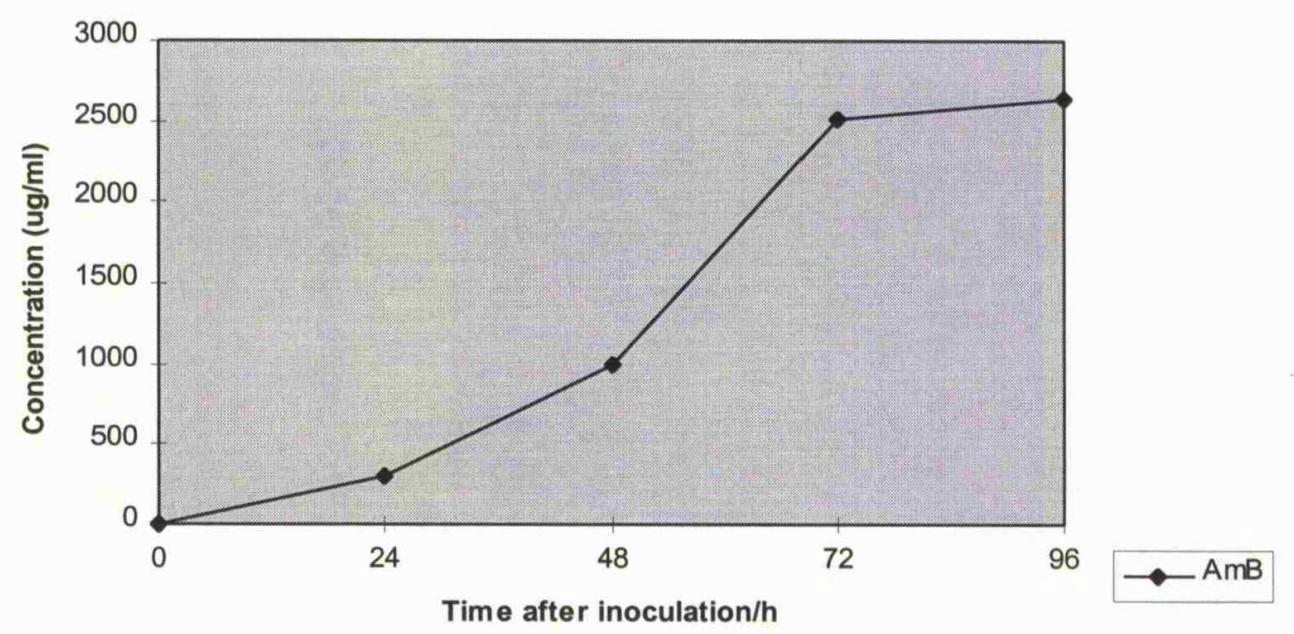

Fig. 3.8 Time-course of amphotericin B production in FCA

media per $500 \mathrm{~cm}^{3} \mathrm{SF}$

(in Leicester). Fig. 3.8 shows the time-course with the different phases of antibiotic production identifiable. The most important aspect was determining the point when amphotericin B had just begun to be produced. This would be an important factor for feeding experiments or indeed the reflasking of the whole broth or the resuspending of cells in a buffer system, which will be described in the next sub-section.

Once a satisfactory fermentation procedure had been formulated in the $500 \mathrm{~cm}^{3}$ shake flasks, experiments were undertaken to establish whether shake flasks of different sizes could be used resulting in similar production levels. The labelled compounds that were to be fed were only available in a limited quantity and so for both the NMR 
and mass spectrometry studies, we needed to maximise the incorporation by feeding to a small a quantity of broth as possible. But what was the lowest volume of broth that would continue to produce amphotericin in satisfactory quantities?

Investigations were undertaken first with $100 \mathrm{~cm}^{3}$ shake flasks, and then with as small as 25 and $10 \mathrm{~cm}^{3}$ flasks. The volume of media was scaled down in direct accordance with the flask size, still ensuring ca. 12\% volume in the flask (in the 100, 25 and $10 \mathrm{~cm}^{3}$ flasks there was approximately 15,5 and $2 \mathrm{~cm}^{3}$ of media, respectively). As the volumes of the fermentation broths that were being handled were much reduced, it was considered that satisfactory inoculation would not be possible with the 25 and $10 \mathrm{~cm}^{3}$ shake flasks, thus a $500 \mathrm{~cm}^{3}$ shake flask was inoculated as usual and was allowed to grow for $24 \mathrm{~h}$ before being reflasked in the smaller vessels under sterile conditions. The amphotericin levels were assayed at regular intervals. For the smaller flasks, the production levels of amphotericin B were low, and the small volumes of whole broth made recovering all the liquid from the flask (by decanting) difficult, and so further studies were only carried out for the $100 \mathrm{~cm}^{3}$ shake flasks. A time-course of amphotericin A and B production can be seen in Fig 3.9. It is clear that the ratio of amphotericin A:amphotericin $B$ has altered, however the titres of the antibiotic calculated by the UV assay were still very encouraging, and thus this fermentation procedure was considered as a viable option. [This result is in line with a result obtained during SB studies, when amphotericin A and $\mathrm{B}$ production in $250 \mathrm{~cm}^{3}$ shake flasks resembled the ratio of $\mathrm{A}: \mathrm{B}$ obtained with $\mathrm{CM}$, as opposed to FCA, in $500 \mathrm{~cm}^{3} \mathrm{SF}($ i.e. $\left.\sim 1: 1)\right]$. 


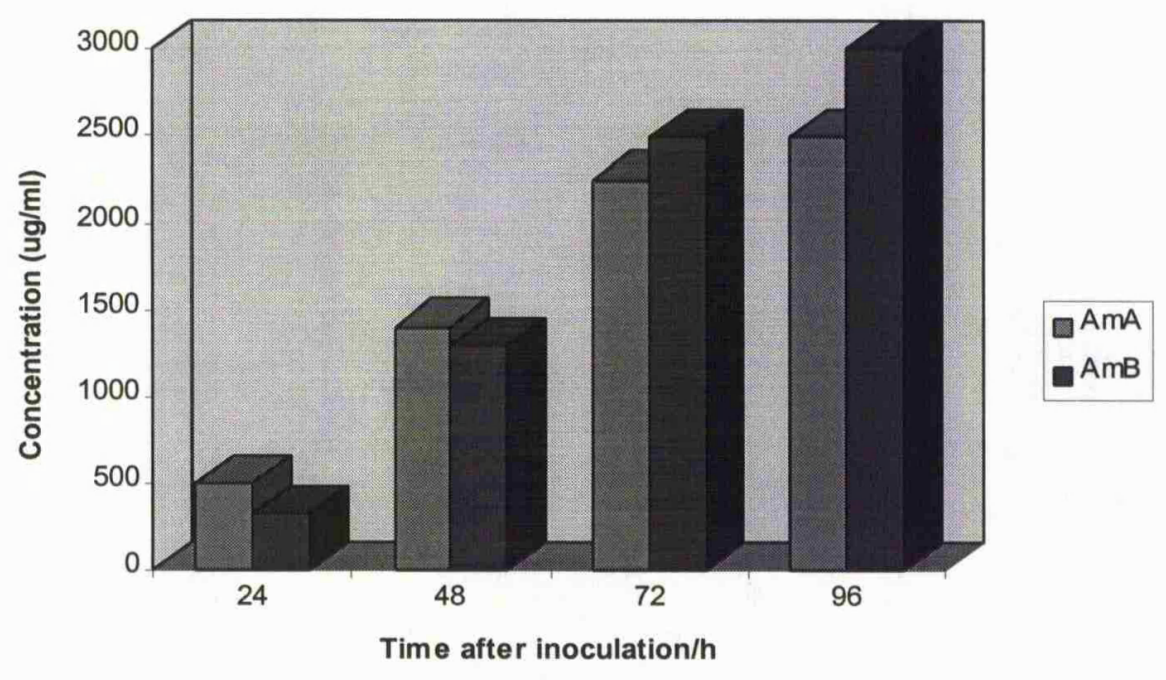

Fig.3.9 Comparison of amphotericin A and B production in FCA media and $100 \mathrm{~cm}^{3}$ shake flasks

Thus, a fermentation process had been developed in which amphotericin B was produced in high quantities. The production of the metabolite in the concentrated media was satisfactory in a variety of volumes of shake flask with corresponding levels of culture broth, any of which could be adopted for labelled feeding experiments (both NMR and mass spectral studies), as described in Chapter 4.

\subsubsection{Resuspension of cells}

For the diketide feeding and the mass spectrometry studies, alternative fermentation methods were investigated in which a small quantity of microorganism would result in the production of relatively large amounts of amphotericin B. 
The resuspension of cells in a buffer system was examined, as it was considered that once the mycelial cells were producing amphotericin, if they were washed and resuspended in buffer, this would remove all the amphotericin B that had already been produced. Once resuspended in buffer, it was to be determined whether the cells would continue producing amphotericin B as usual. If so, it was considered that feeding the labelled compounds to this system would result in an increased possibility of detecting incorporation because much of the 'background' levels of amphotericin B (i.e. produced prior to the feeding of labels) would have been removed.

Similar studies undertaken in this field with different species of microorganisms involved procedures that made the use of replacement cultures. The first reported use of replacement cultures, in combination with high glucose concentrations, was by Vederas et al. when investigating the biosynthesis of dehydrocurvularin. ${ }^{34}$ Vegetative cultures were initiated by inoculation of the medium with seed culture as usual, which were allowed to grow and produce for $24-48 \mathrm{~h}$. The broth was then centrifuged under sterilised conditions and the mycelia transferred into autoclaved flasks containing the replacement media, which consisted of a much higher percentage of glucose than the original production medium, along with normal levels of the specified substrates and ionic salts. In the labelled feeding studies of dehydrocurvularin, which is excreted from the cells, using replacement media removes any unlabelled metabolite that is produced prior to the feeding of the metabolite. A high glucose medium was used in particular, as it was considered high 
glucose levels might suppress $\beta$-oxidation. ${ }^{85}$ This method has also been utilised in studies with $S$. venzualae in the biosynthesis of methymycin, nargenicin and erythromycin..$^{32,35,149}$ For all these examples, the replacement media that was used was, in essence, a type of defined media, with higher than normal levels of glucose but similar levels of salts and nutrients etc. However, as discovered earlier, $S$. nodosus did not grow or produce satisfactorily in such defined media and so this type of replacement media was not investigated. A replacement media that had acetate as the carbon source was investigated in the feeding studies, however $S$. nodosus did not grow or produce well in this media, as mentioned in Chapter 4 .

Instead, a study was undertaken in which the cells of the whole broth were washed and resuspended in a buffer system. It was considered that the process would ultimately increase the possibility of detecting the incorporation of the added precursor, by removing much of the amphotericin B that was produced prior to the washing of the cells, as in the replacement media. The use of a buffer system would also result in the removal of much of the media debris when the mycelial cells were washed with the buffer, which might also increase the possibility of any detection of incorporation. Therefore, investigations were carried out to determine whether amphotericin production would in fact continue once the cells were resuspended in buffer, without the presence of the carbon and nitrogen sources, with a possible determining factor for the success of these investigations being the actual time of resuspension. 
Thus, the groundwork for the washed cells experiment involved determining which buffer system to use and also working out the precise methodology for the experiment, i.e. it was important to use a centrifuge speed that would spin down the whole broth, but one that did not result in a pellet that was too compact to be resuspended. Initial studies were carried out with MOPS [3-( $N$-morpholine)propanesulfonic acid] and PBS (phosphate buffered saline) buffer systems, however MOPS produced more amphotericin and resulted in a more satisfactory pellet than PBS and thus was used in further studies.

The general procedure for the washed cells experiment which was formulated during my placement at SmithKline Beecham Pharmaceuticals can be seen in Fig.3.10. For this procedure, the microorganisms were grown in $250 \mathrm{~cm}^{3}$ shake flasks. The production medium was inoculated as usual with the primary seed, and was shaken under usual conditions for a specified period. The optimum time to resuspend the whole broth was one factor that had to be determined. In order to investigate this, production flasks containing FCA media were removed from the shaker 24, 48 and $72 \mathrm{~h}$ after inoculation ( 3 flasks per day), the cells washed and resuspended in buffer and returned to the shaker for a further $72 \mathrm{~h}$. The concentration of amphotericin was measured in the initial whole broth, as well as after resuspension $(N)$ and thereafter for three consecutive days $(N+24 \rightarrow N+72)$ (Fig. 3.11). Thus, it was determined whether the cells continued to produce amphotericin once they were transferred to the buffer system. The concentrations of amphotericin B in the whole broth at the times of resuspension were progressively higher in those flasks resuspended at 24, 48 


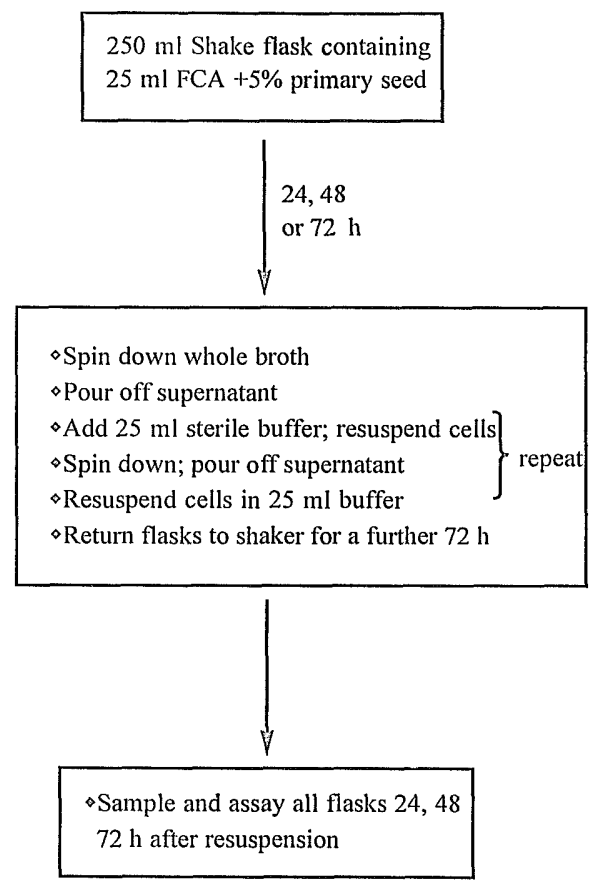

Fig. 3.10 General procedure for washed cells experiment

and $72 \mathrm{~h}$ after inoculation (ca. 200, 1400 and $2200 \mu \mathrm{g} / \mathrm{ml}$, respectively) and thus the values of the cells initially resuspended in buffer (at $t=N \mathrm{~h}$ ) were correspondingly higher.

The value of $N$ indicated that about half the concentration of amphotericin must have been associated with the supernatant in the calculation of the whole broth titres, which was removed with the washing with buffer. In Fig. 3.12 values can be seen for 


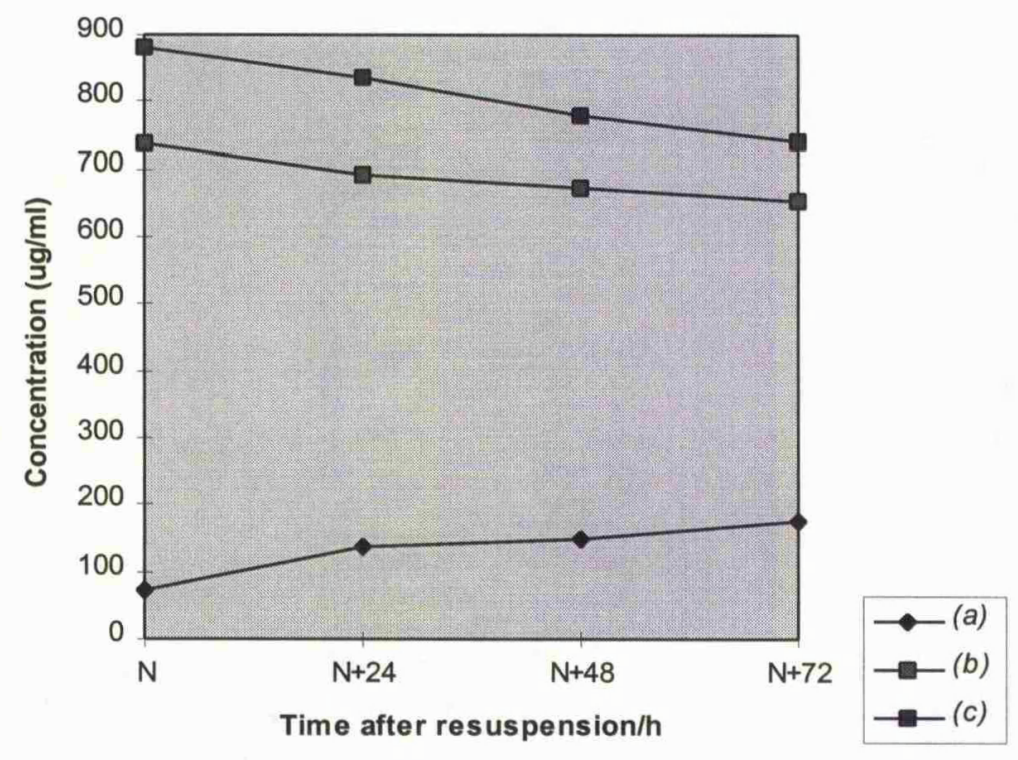

Fig.3.11 Resuspension of cells in MOPS buffer; $N=$ time of resuspension, (a) $N=24 \mathrm{~h},(b) N=48 \mathrm{~h}$ and (c) $N=72 \mathrm{~h}$

the assay of the whole broth prior to removal from the shaker, along with an assay of the two washings with buffer, and then finally once resuspended and returned to the shaker. As mentioned previously, the $250 \mathrm{~cm}^{3}$ shake flasks resulted in a ratio of amphotericin A : amphotericin B of 1:1, as can be seen in Fig.3.12. The antibiotic levels at the different stages, indicate that about half the original level of amphotericin in total was lost in the washings, with the amphotericin present in the buffer system after resuspension (at $t=N \mathrm{~h}$ ) being associated with the mycelial cells. In Fig. 3.11 it can be seen that only the cells resuspended in buffer $24 \mathrm{~h}$ after inoculation continued to produce amphotericin once returned to the shaker, in which the levels of amphotericin B actually more than doubled once resuspended in the buffer system (Fig. 3.13). Thus, it was shown that the most increase in production 


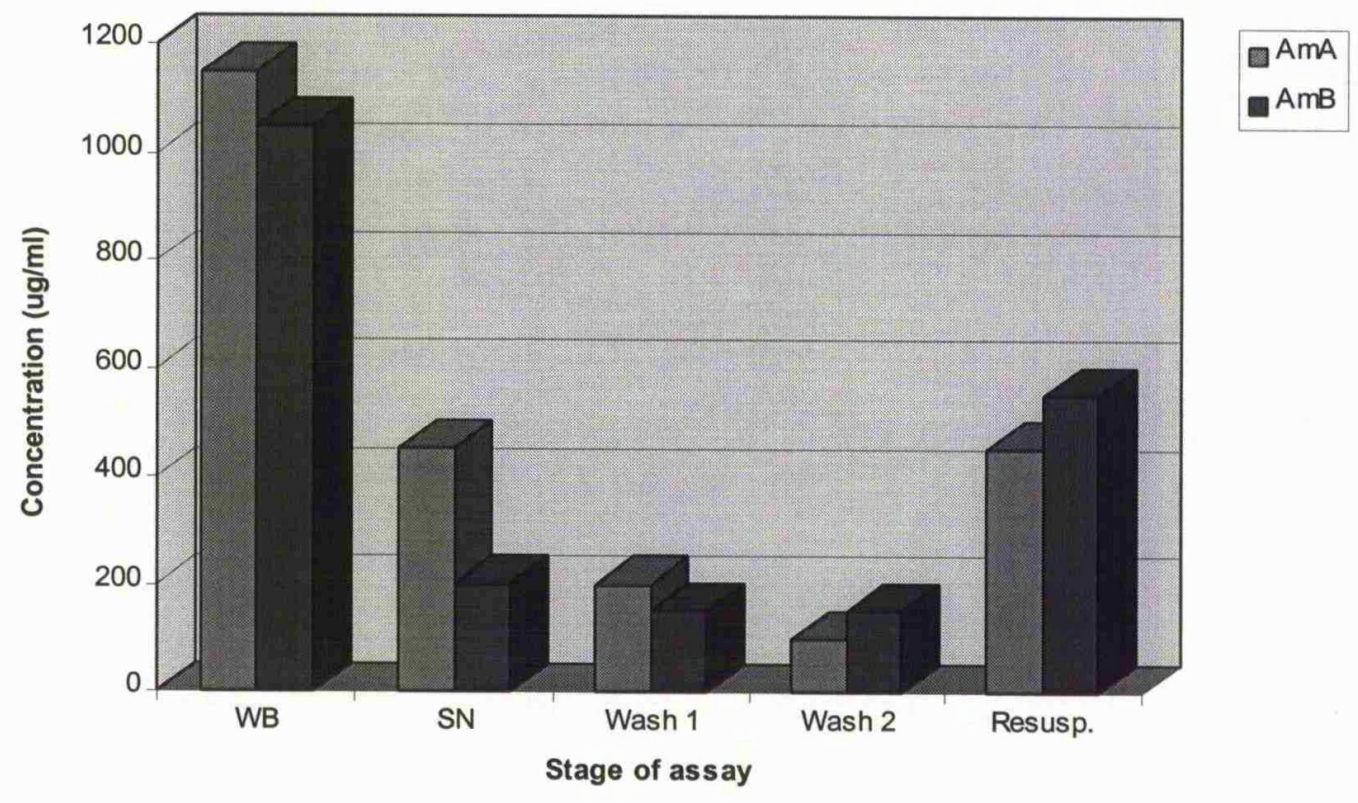

Fig. 3.12 Comparison of amphotericin A and B concentration at the different stages of the wash cells experiment, for cells resuspended $72 \mathrm{~h}$ after inoculation

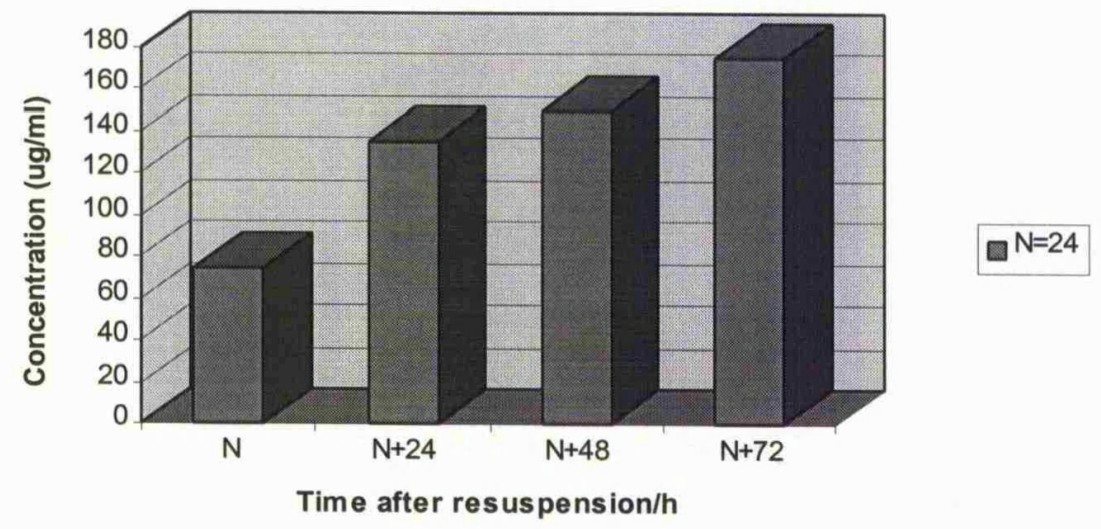

Fig. C13 Resuspension of cells in MOPS buffer $24 \mathrm{~h}$ after inoculation 
once resuspended in buffer, was in the first day after resuspension, between 24 and $48 \mathrm{~h}$.

The next study involved a more detailed investigation involving washing and resuspending the cells between 0 and $48 \mathrm{~h}$. It was considered there would be little amphotericin production between 0 and $12 \mathrm{~h}$, as this was the lag phase, in which the microorganism would be growing and adjusting to the environment. Thus, cells were washed, resuspended and assayed between 13 and $49 \mathrm{~h}$ after inoculation on a four hourly basis. Each flask was then assayed 24 and $48 \mathrm{~h}$ after resuspension to see whether amphotericin production continued. In all the flasks, there was increase in concentration of amphotericin after $24 \mathrm{~h}$ (Fig. 3.14, the difference between the $N$ and

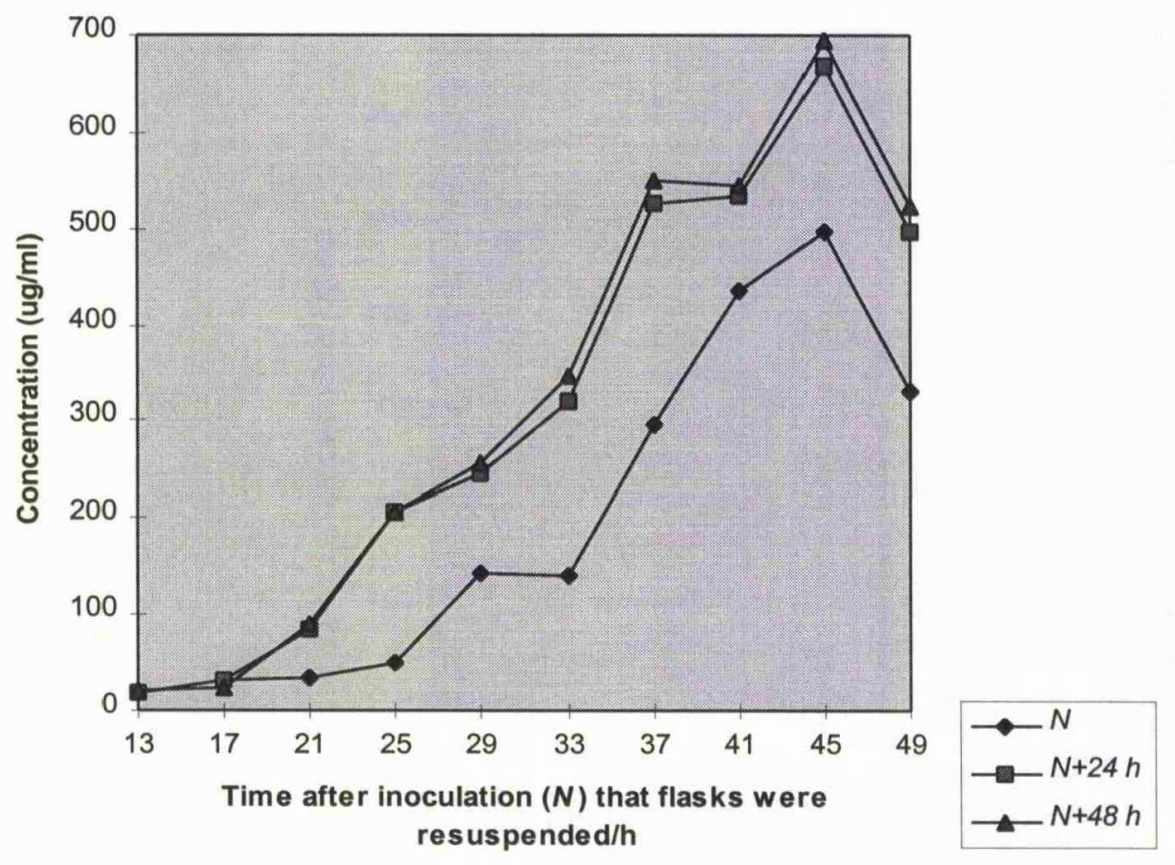

Fig. 3.14 Comparison of amphotericin B concentration in flasks resuspended at different times 
the $N+1$ values), but the percentage increase varied among the flasks, depending on the time of resuspension. With the cells aged between 21 and $25 \mathrm{~h}$ old at the time of resuspension, there was a four fold increase $24 \mathrm{~h}$ after being resuspended in the buffer system. For the 29-37 h flasks, there was a doubling of amphotericin concentration, and after this the percentage increase in amphotericin production between $N$ and $N+1$ became less. There was no significant increase one day later $(N+2)$ in all flasks. Thus, for the washed cells experiment, once resuspended, the cells only continued to produce amphotericin for up to $24 \mathrm{~h}$. This would be adequate for the feeding of precursors, which would be expected to be taken up by the cells in this time period. The final point to be clarified was exactly when to wash the cells and resuspend in buffer. It seems that the preferable time would be $30-35 \mathrm{~h}$ after inoculation; the borderline region of 25-29 h would not be ideal as there would be a possibility that the amphotericin B concentration on a certain day would be at the lower end of the scale.

On returning to Leicester, the washed cells investigations were continued, however, at that time, the FCA media was unavailable and so experiments were undertaken in the complex media. This resulted in much lower titres of amphotericin in the production medium, and although the levels of amphotericin were doubled $24 \mathrm{~h}$ after being resuspended, as expected, the actual concentration levels were much lower, which would make the extraction and purification of the amphotericin very difficult. It was due to the difficulties encountered in the extraction and purification process, as a result of the low levels of metabolite, that a halt was called to this line of 
investigation until a certain time when such low concentrations of amphotericin could be extracted and purified satisfactorily.

\subsection{Extraction and punification}

The isolation of many extracellular secondary metabolites from the fermentation broth often begins with the major problem of preliminary cell and insoluble media components separation. For the extraction of amphotericin B, previous studies have involved successive extractions of the whole broth with butanol which were then centrifuged to remove any insoluble components. This combined extract was then concentrated, cooled and a specified volume of diethyl ether added. Both amphotericin $\mathrm{A}$ and $\mathrm{B}$ consequently precipitated out and were later separated by countercurrent distribution (CCD)..$^{148}$

For our studies with amphotericin B, the extraction and purification process was governed by the method of analysis to be used, either electrospray mass spectrometry (ESMS) or NMR. The aim of this work was to gain as much information as possible about the biosynthetic processes involved in the production of amphotericin B by the addition of variously labelled compounds. As mentioned previously, the feeding of the labelled diketide with incorporated deuterium labels was to be used in collaboration with electrospray mass spectrometry, for which only a small sample would be required (up to $1 \mathrm{mg}$ ). However, initial feeding experiments were to be carried out which incorporated ${ }^{13} \mathrm{C}$ labels, and so the most desirable method of 
analysis in this case would be NMR, for which $30 \mathrm{mg}$ of substrate would need to be isolated in a sufficiently pure form. Thus, the different feeding experiments would require different growth conditions, as discussed already, but also different methods of extraction and purification.

\subsubsection{Electrospray mass spectrometry}

The procedure used for samples to be analysed by mass spectrometry involved solvent extraction followed by purification by high performance column chromatography (HPLC). The solvent extraction would result in both amphotericin $\mathrm{A}$ and $\mathrm{B}$ being extracted to give a crude mixture that could be purified by HPLC with detection at different wavelengths (305 $\mathrm{nm}$ for amphotericin A and $405 \mathrm{~nm}$ for amphotericin B). Initial studies to determine an adequate procedure involved analytical HPLC, however when crude mixtures were injected and the specific peaks were collected, a semi-preparative procedure was followed.

The mobile phase used in conjunction with the HPLC technique was developed during my time spent at SmithKline Beecham Pharmaceuticals. To ascertain the correct conditions, e.g. flow rate, ratio of buffer : solvent, attenuation of peaks etc, solutions of known concentration of amphotericin B were made up and injected onto the HPLC (for a more detailed outline of the procedures used, see Experimental section). Two possible mobile phase systems included (a) 80:20, methanolmonosodium phosphate buffer $(0.05 \mathrm{M}, \mathrm{pH} 3.5$ adjusted with phosphoric acid) and (b) 73:27, methanol-sodium citrate buffer ( $0.05 \mathrm{M}$, pH 5.3 adjusted with citric acid), both 
of which involved the ionisation of the $\mathrm{NH}_{2}$ group of the amphotericin to facilitate the movement of the amphotericin down the reverse-phase column. The first buffer system was not satisfactory if the sample was to be analysed by HPLC, as the phosphate buffer renders the solution unsuitable for mass spectrometry. Thus, the sodium citrate buffer was used in conjunction with methanol, the ratios of each in the mobile phase would determine the retention time of the desired peaks. Increasing the ratio of methanol would cause the substrate to run off the column faster, as would increasing the flow rate. In Fig. 3.15 is a HPLC trace after a $100 \mu 1$ injection of a 100 $\mu \mathrm{g} \mathrm{cm}^{-3}$ solution of standard amphotericin B, measuring at a wavelength of $405 \mathrm{~nm}$.

To evaluate all the processes involved from extraction to analysis, an ESMS was next run on a known sample of amphotericin. The electrospray technique is the first choice for large protonatable biomolecules, such as proteins and enzymes, but can

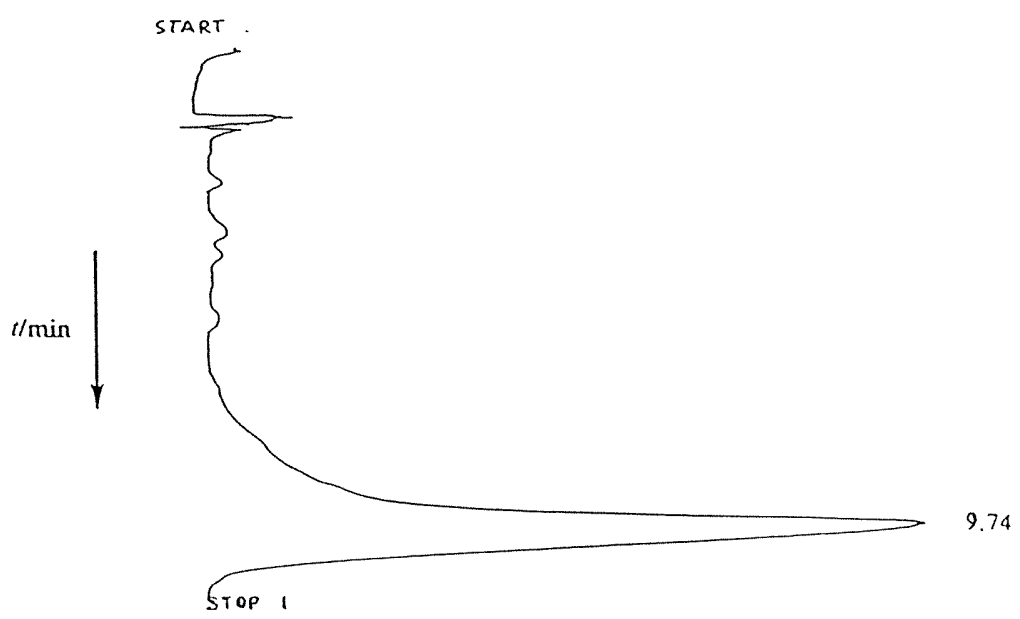

Fig.3.15 HPLC trace of a sample (10 $\mu \mathrm{l})$ of standard amphotericin B solution $\left(100 \mu \mathrm{g} \mathrm{cm}^{-3}\right)$ 
also be used for large metabolites such as amphotericin, and provides an accurate method for measuring incorporation. The spectrum obtained from a standard amphotericin B solution (Fig.3.16) is much improved over previous mass spectrometric techniques such as $\mathrm{FAB}$ (fast atom bombardment) which did not provide any satisfactory spectra. The spectrum acquired is exactly as what would be expected, as can be seen by comparing this spectra with the theoretically calculated (inset, Fig.3.16). The expected mass peak of 924 is evident, with the additional peak distribution corresponding to the natural abundance of ${ }^{13} \mathrm{C}$ in the molecule, which is as expected for a compound consisting of $c a$. 50 carbon atoms (1\% natural abundance of ${ }^{13} \mathrm{C}$ ). This technique is ideal for the detection of incorporation of the labelled diketide, as if it is incorporated intact, there will be an increase in intensity of the $\mathrm{M}+4$ peak. If however, the diketide is reoxidised prior to incorporation into the metabolite resulting in the loss of a deuterium, an increase in intensity at the $M+3$ peak will be detected.

The initial investigations for this procedure involved the extraction into hot butanol $\left(3 \times 100 \mathrm{~cm}^{3}\right)$ of whole broth $\left(60 \mathrm{~cm}^{3}\right)$ from $500 \mathrm{~cm}^{3}$ fermentation flasks. The extracts were centrifuged to separate the insoluble mycelia and media components, and the resulting yellow solvent solutions were evaporated under reduced pressure, with any remaining water being removed by freeze drying. These extractions resulted in crude mixtures that were redissolved in the smallest amount of methanol. The crude mixture was assayed and the resulting UV spectrum can be seen in Fig. 3.17. From this UV assay, the relative amount of amphotericin $A$ and $B$ in the 


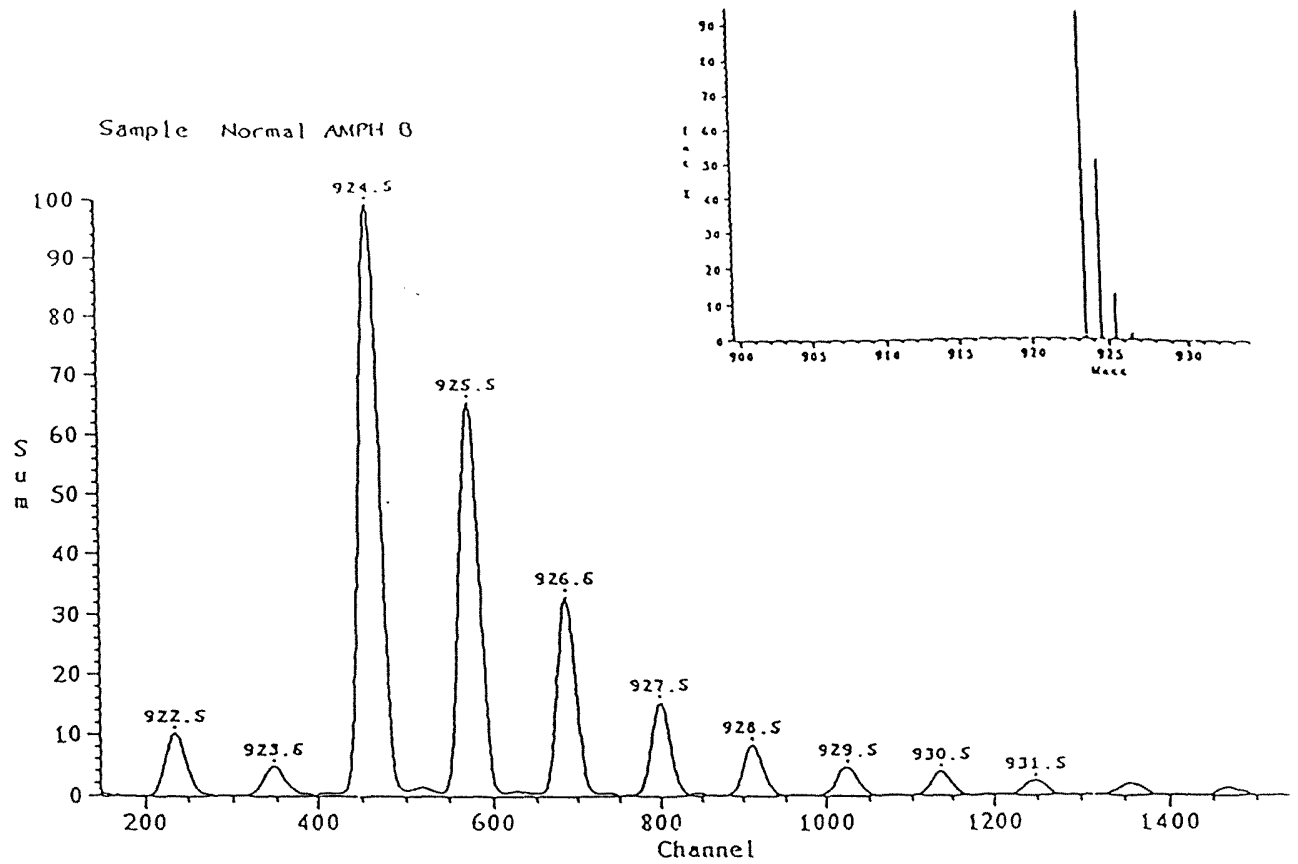

Fig. 3.16 Electrospray mass spectrum of a standard sample of amphotericin B collected after

injection onto the HPLC (maximum resolution). Inset: theoretical calculation

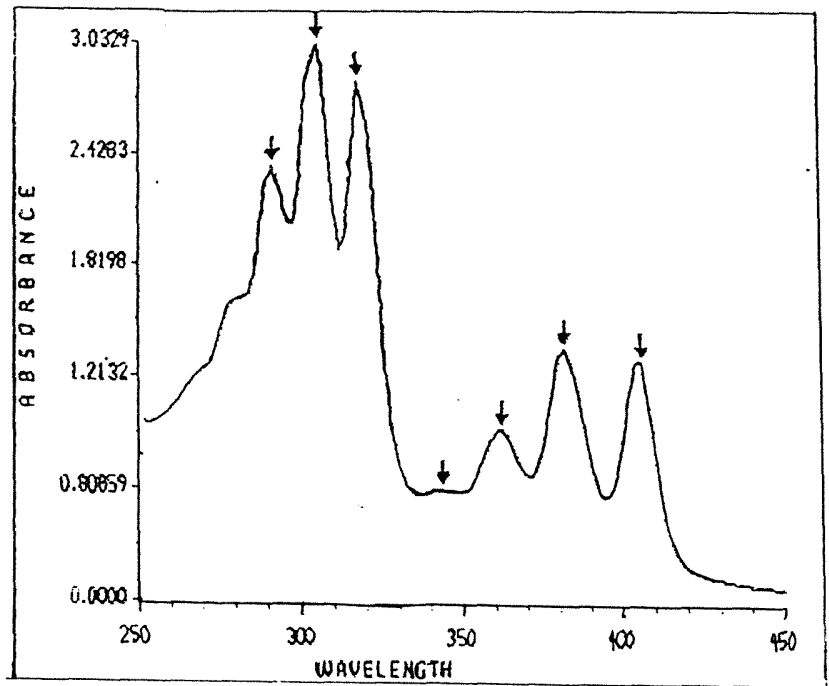

Fig. 3.17 UV assay of crude butanol extract; $\lambda_{\max } 305 \mathrm{~nm}$ for $A m A$ and $\lambda_{\max } 405 \mathrm{~mm}$ for $\mathrm{AmB}$ 
mixture could be determined as described previously, to give a relative indication of the concentration of each,. Samples of the mixture were then injected onto the HPLC and once conditions etc. had been formulated, the analytical column was exchanged for a semi-preparative one. Successive aliquots of $500 \mu \mathrm{l}$ were injected onto the column and the appropriate fractions collected. The HPLC traces at 305 and $405 \mathrm{~nm}$ can be seen in Fig. 3.18, with amphotericin A having a retention time of 5 min and amphotericin B ca. 9 min. The initial peaks in both traces are due to the solvent fronts, which were of large intensity due to the required increased attenuation settings. Due to the slight fluctuations the ambient temperatures of the column, differing retention times were obtained on subsequent days, however a standard solution of amphotericin $\mathrm{B}\left(100 \mu \mathrm{g} \mathrm{cm}^{-3}\right)$ was injected routinely in order to elucidate

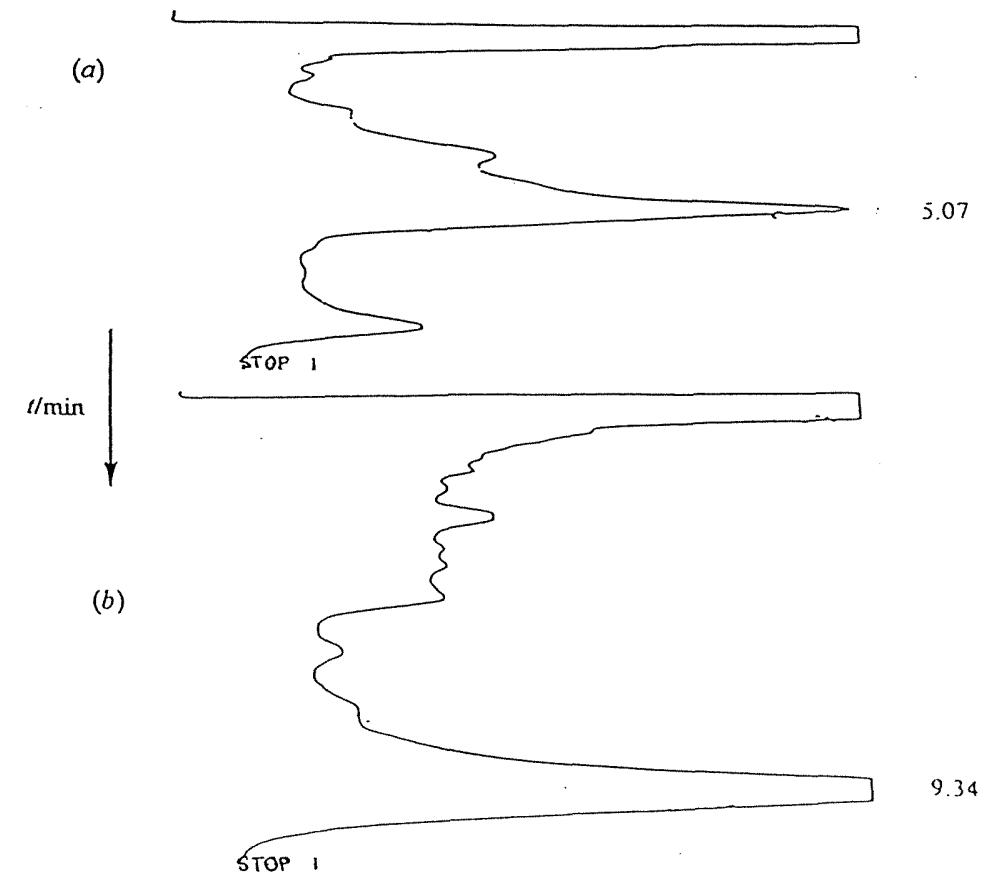

Fig. 3.18 HPLC trace of butanol cxtracts (a) at $305 \mathrm{~mm}$ and (b) at $405 \mathrm{~mm}$ 
the conditions for that particular day. The peaks of amphotericin A and B were collected and the fractions were concentrated in vacuo. The yellow residue obtained for each compound was then redissolved in the smallest amount of methanol and subject to electrospray mass spectrometry. Satisfactory mass spectra were obtained that were comparable to the standard example with the collected amphotericin B having a mass peak of 924 and the amphotericin A, a mass peak of 926 .

The next step, in order to reproduce this extraction and purification procedure, was to attempt the process using smaller volumes of whole broth. Fermentations were undertaken in $100 \mathrm{~cm}^{3}$ shake flasks containing $c a .15 \mathrm{~cm}^{3}$ of production medium. Once again, after a number of days on the shaker, the whole broth was extracted into hot butanol $\left(3 \times 50 \mathrm{~cm}^{3}\right)$. This was repeated a number of times and the resulting extracts were pooled and the solvent evaporated, to give a yellow residue that was redissolved in a minimum amount of methanol. This solution was injected onto the HPLC and the appropriate peaks collected. Unfortunately, the small scale samples that were collected from the HPLC and redissolved in a small amount of methanol did not produce suitable a satisfactory mass spectrum. This was probably due to the fact that there was a high spray current, possibly because of the presence of citrate salts from the HPLC buffer which interfered with the detection process. A variety of methods were used in an attempt to de-salt the system, including the use of a size exclusion column (LH20). However, an adequate mass spectrum was still unattainable which must have meant the size exclusion column was not able to adequately separate the amphotericin from the salts. 
Adsorption column chromatography was then attempted. Adsorption processing using neutral non-polar macroporous resins for the separation of biochemical products is used widely. For this process, the peaks that were collected from the HPLC and redissolved in a small amount of methanol were loaded onto the adsorption column (HP20) and deionised water was then run through the column, which resulted in the citrate salts eluting while the amphotericin molecules remained at the top of the column due to the adsorbing interactions. The eluting mobile phase of water was then exchanged for methanol which resulted in the amphotericin moving down the column with the solvent front.

The fraction containing the amphotericin $\mathrm{B}$, which was detected by an attached UV monitor, was collected and the solvent evaporated under reduced pressure. The resulting yellow residue was redissolved in a small amount of methanol and subsequently produced a satisfactory electrospray mass spectrum. However, this extraction and purification procedure did not reliably produce consistently adequate mass spectra. One reason for this was the low levels of amphotericin extracted from the $15 \mathrm{~cm}^{3}$ of whole broth. It is important to note that samples of as low as $50 \mu \mathrm{g}$ $\mathrm{cm}^{-3}$ of a standard amphotericin solution produced satisfactory spectra consistently, however it seemed that a greater concentration of $\mathrm{AmB}$ in solution was required than many other compounds that were subjected to ESMS. Thus, the solvent extraction was further investigated in order to increase the concentration of metabolite extracted from the whole broth. 
Hot ethyl acetate and methanol extractions of the whole broth were attempted, along with a methanolic extraction of freeze dried whole broth. As the volumes of whole broth were so small it might have been advantageous, to ensure the maximum amount of amphotericin B was extracted, if the water was first removed. For this, the culture fluid was transferred from the shake flask into a round bottom flask and subject to freeze drying (reduced pressure, $-30^{\circ} \mathrm{C}$ ) overnight.

The resulting yellow solid was then subject to successive extractions with methanol. The yellow solid that resulted from the freeze drying process were extracted six times with methanol (each of $20 \mathrm{~cm}^{3}$ ), the assays of each of which can be seen in Table 3.1. The extracts were then pooled and evaporated to dryness. The solid that remained was redissolved by sonicating for $20 \mathrm{~min}$ in methanol and DMSO $\left(3\right.$ and $1 \mathrm{~cm}^{3}$, respectively; as amphotericin $\mathrm{B}$ dissolves readily in DMSO, a portion was added in order to redissolve the maximum amount of metabolite; extract 1). However, all the solid did not dissolve and so the mixture was centrifuged, the supernatant removed and the remaining solid redissolved by sonicating for $20 \mathrm{~min}$ in methanol and DMSO (2 and $0.5 \mathrm{~cm}^{3}$, respectively; extract 2). The two extracts were then injected onto the HPLC separately, with detection at both 305 and $405 \mathrm{~nm}$. The HPLC traces show a great improvement over previous extractions with large quantities of amphotericin $\mathrm{A}$ and $\mathrm{B}$ present (with extract 1 containing the majority of $\mathrm{AmA}$ and extract 2 containing mainly AmB) (Fig. 3.19). 
Table 3.1 UV assay of freeze dried whole broth methanol extractions from FCA fermentations in $100 \mathrm{~cm}^{3} \mathrm{SF}$

\begin{tabular}{lll}
\hline Extraction & Concentration $/ \mu \mathrm{g} \mathrm{cm}^{-3}$ & \\
& Amphotericin $\mathrm{A}^{a}$ & Amphotericin $\mathrm{B}^{b}$ \\
\hline Initial WBc & 1825 & 2895 \\
1 & 1475 & 2140 \\
2 & 3391 & 4825 \\
3 & 1145 & 2912 \\
4 & 5233 & 3985 \\
5 & 971 & 1259 \\
6 & 160 & 183 \\
\hline
\end{tabular}

$a_{\text {Detected at } \lambda 305 \mathrm{~nm} .}{ }^{b}$ Detected at $\lambda 405 \mathrm{~nm} .{ }^{c} \mathrm{WB}=$ whole broth.

The appropriate fractions were collected, de-salted and the samples redissolved in a small amount of methanol $\left(0.5 \mathrm{~cm}^{3}\right)$ and injected onto the ESMS, which resulted in excellent spectra, which were reproducible. Thus, a satisfactory extraction and purification procedure for both amphotericin $A$ and $B$ from small volumes of whole broth has been formulated. This detailed investigation will provide invaluable groundwork for future investigations into the production of amphotericin B by $S$. nodosus. 


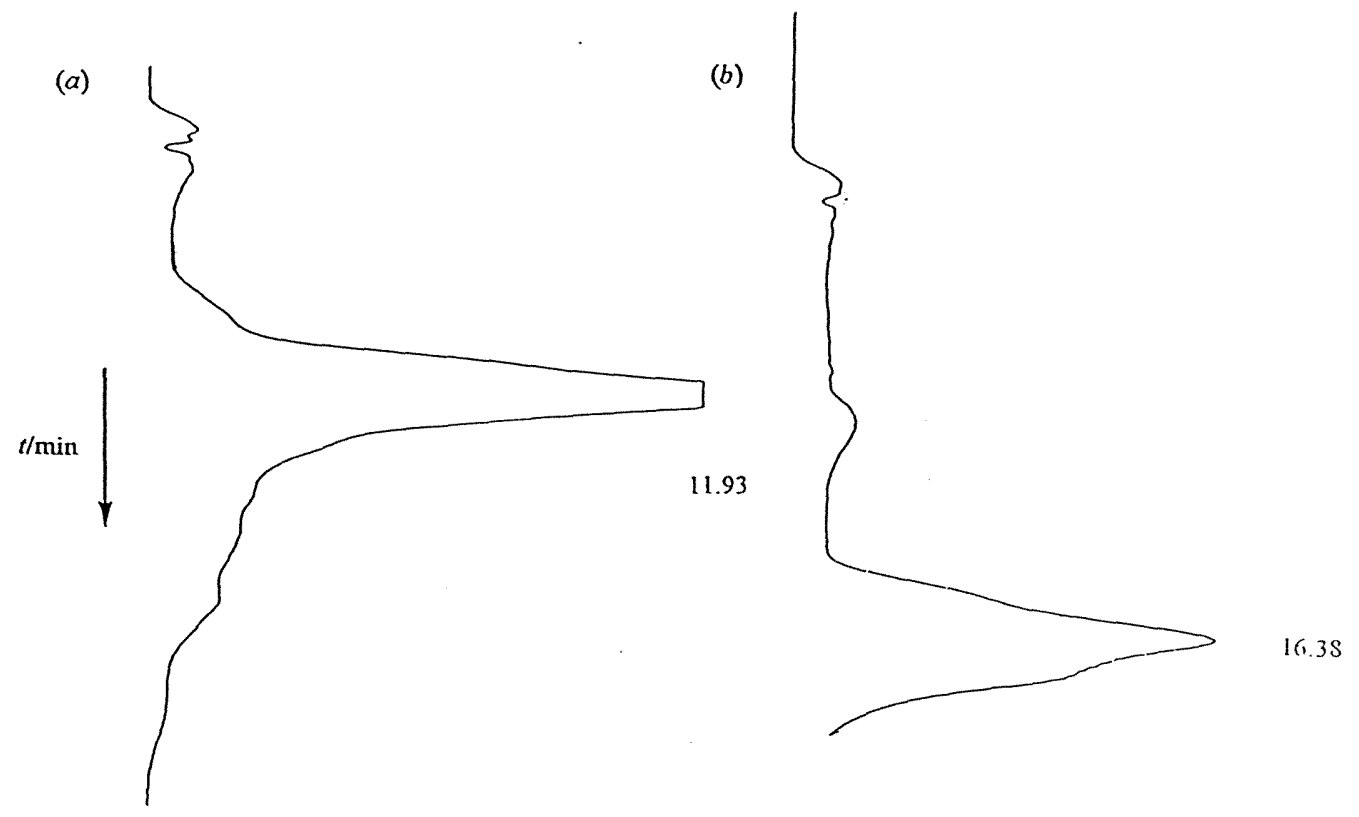

Fig. 3.19 HPLC trace of $(a)$ extract 1 detecting at $\lambda 305 \mathrm{~nm}$ and

(b) extract 2 detecting at $\lambda 405 \mathrm{~nm}$ 


\subsubsection{NIMR spectroscopy}

For the extraction of suitable quantities of metabolite from the whole broth for analysis by NMR (20-30 mg), a modified solvent extraction was utilised. The procedure was developed at SmithKline Beecham Pharmaceuticals and involves the use of a liquid ion exchange isolation. ${ }^{150}$ This process involves the use of a hydrophobic extractant ion, which is supplied as an organic solvent solution, which exchanges its counter ions with an ion in the aqueous phase. The success of this process is dependent on the target compound in the aqueous phase possessing an ionisable functional group, as in the case of amphotericin which has both a carboxylic acid group and an amine, which results in the formation of an ion pair.

The original extraction procedure involved the initial adjustment of the whole broth $\mathrm{pH}$ to 10.5 with sodium hydroxide solution $(5 \mathrm{M})$, under which conditions the amphotericin would be present in its anionic form. This then interacts and is extracted by the cationic reagent, in this case Aliquat 336 (a commercial form of trioctylmethyl ammonium chloride) which is present as a $7 \%(\mathrm{w} / \mathrm{v})$ solution in the water immiscible organic solvent. The formation of the ion pair between the Aliquat and amphotericins A and B, greatly increases their solubility in the organic solvent, thus enhancing the extraction. The original procedure by SmithKline Beecham ${ }^{150}$ utilised the solvent butanol preferentially with which the whole broth was vigorously agitated for $30 \mathrm{~min}$, during which time the $\mathrm{pH}$ was maintained at 10.5 . The phases were then separated by centrifugation, retaining and assaying the organic phase to 
determine the content of amphotericin. In order to recover the amphotericin $B$, the extract was neutralised in order for the amphotericin to be reprotonated, thus breaking the ion pair and causing the amphotericin to precipitate. In the SmithKline Beecham procedure this was done by the addition of ethyl acetate $(1 \% \mathrm{v} / \mathrm{v})$ to the Aliquat-amphotericin conjugate solution, which then slowly underwent saponification due to the trace amounts of sodium hydroxide present in the solution. The acetic acid produced resulted in a gradual lowering of the $\mathrm{pH}$, protonating the amphotericin B which then crystallised as distinct spherulites. The precipitate that resulted over 3-4 days due to the slow crystallisation of amphotericin B was easily recovered by resuspension in the mother liquor, filtration and washing with water, acetone and methanol and was identified by NMR to be amphotericin B of high purity $(>95 \%)$, with no detectable presence of amphotericin A.

Due to the lower titres of metabolite obtained for fermentations carried out in Leicester, a similar liquid ion exchange process was followed, however ethyl acetate was found to be the most appropriate extraction solvent. Following the extraction, once again the phases were separated and the organic phase retained. As the organic solvent in this extraction was ethyl acetate, saponification of the ion pair occurred without the need for an external initiator. A UV assay to determine the amphotericin content of the extract initially was carried out, which clearly shows the presence of both amphotericin A and B (Table 3.2). The precipitation of amphotericin B then proceeded satisfactorily over 3-4 days, after which the bright yellow precipitate was 
Table 3.2 UV assay of the precipitation process of amphotericin B from an ethyl acetateAliquat extract of a whole broth sample $\left(60 \mathrm{~cm}^{3}\right)$ from an FCA fermentation procedure in $500 \mathrm{~cm}^{3} \mathrm{SF}$

\begin{tabular}{llll}
\hline Day & $\mathrm{pH}$ & Concentration $/ \mu \mathrm{g} \mathrm{cm}^{-3}$ & \\
& & Amphotericin $\mathrm{A}^{a}$ & Amphotericin $\mathrm{B}^{b}$ \\
\hline 1 & $9.5-10$ & 1023 & 1160 \\
5 & 7 & 1020 & 253 \\
5 & 7 & 1024 & 253 \\
\hline
\end{tabular}

a Detected at $\lambda 305 \mathrm{~nm}$. $b$ Detected at $\lambda 405 \mathrm{~nm}$.

recovered $(200 \mathrm{mg})$ and identified by ${ }^{13} \mathrm{C} N \mathrm{NMR}$, which gave spectra identical to those previously published. ${ }^{151,152,153,154}$

This extraction procedure was highly selective and resulted in amphotericin B of high purity, although why amphotericin A was not co-precipitated is still unclear. Thus, a required method of extraction and purification of amphotericin $B$ on a larger scale has also been formulated. This method has proved satisfactory with the extraction of large volumes of fermentation broth but the success does appear to have a limit as to the smallest volume that can undergo satisfactory extraction. In our investigations, the contents of one $500 \mathrm{~cm}^{3} \mathrm{SF}\left(60 \mathrm{~cm}^{3}\right.$ of whole broth) underwent extraction and precipitation satisfactorily, however volumes less than this did not precipitate sufficient amounts of amphotericin B for an NMR spectra, and also, the smaller amounts precipitated were more difficult to recover. 


\subsection{Search for minor co-metabolites of amphotericin}

In parallel to the above biological studies, a separate investigation was undertaken into the composition of other metabolites in the fermentation medium. Various studies into the biosynthesis of secondary metabolites have involved the search for intermediates, present in small quantities, in the biosynthetic pathway. These may take the form of simple extraction and purification of the whole broth, or may involve the aid of inhibitors or mutants which block the formation of the final metabolite, resulting instead in the accumulation of enzyme-free intermediates and shunt metabolites. ${ }^{25,26}$ As explained in the Introduction (Chapter 1), in the biosynthesis of polyketides the growing acyl chain is covalently connected to the polyketide synthase until it is of the required length, at which point it is released and is then adjusted by 'tailoring' enzymes via a series of discrete enzyme-free intermediates to eventually produce the desired final metabolite (Fig. 3.20). The isolation and structural elucidation of these later intermediates will indicate the biosynthetic route taken (e.g. at what stage does glycosidation occur?) and these intermediates, in their own right, may have improved medicinal properties over amphotericin B.

In the study of the biosynthesis of amphotericin $\mathrm{B}$, the search for minor metabolites in the fermentation broth that has not been reported previously. The initial step of such a study involved the growth of fermentation cultures of $S$. nodosus $\left(240 \mathrm{~cm}^{3}\right.$ of 


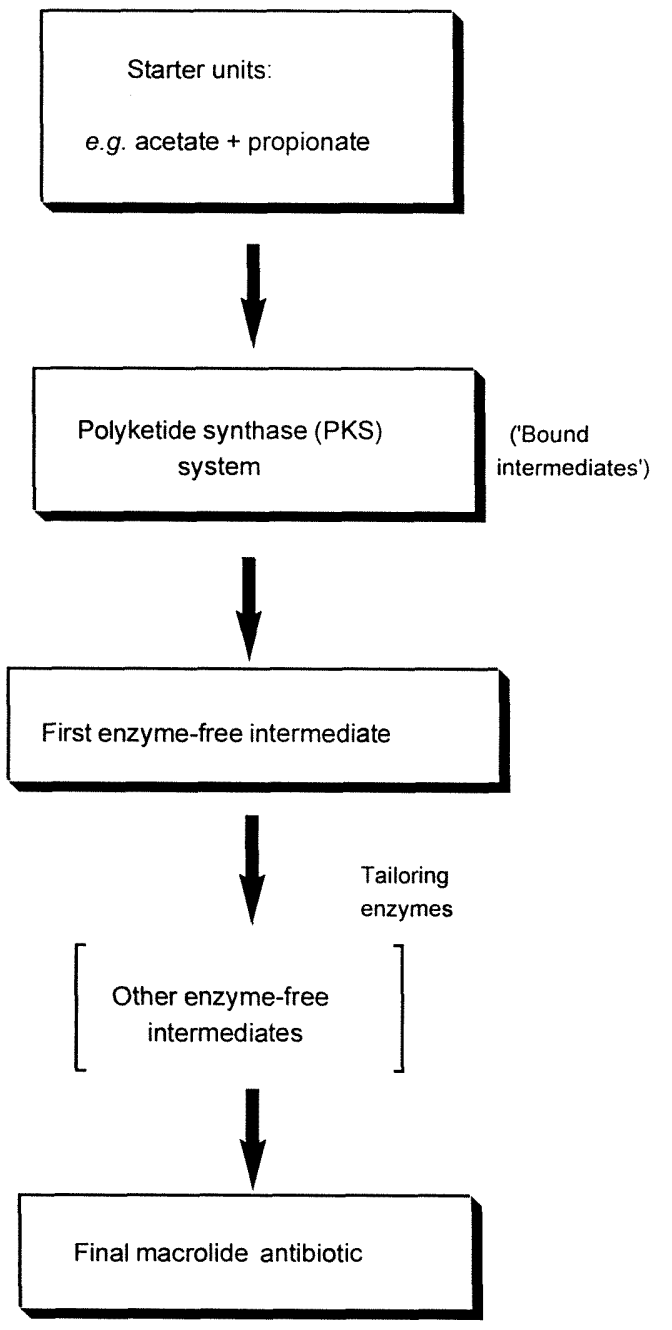

Fig. 3.20 Summary of macrolide biosynthesis 
FCA media in each of $2 \times 2 \mathrm{dm}^{3}$ shake flasks) for 4 days (with the production of amphotericin $\mathrm{A}$ and $\mathrm{B}$ at usual levels), followed by subsequent extraction of the whole broth with butanol $\left(2 \times 500 \mathrm{~cm}^{3}, 1 \mathrm{~h}\right.$ at $\left.c a .50^{\circ} \mathrm{C}\right)$. This extraction process was different to those previously described, as a quantity of citric acid was added during the extraction process in order to enhance the solubility of any basic metabolites present by forming their citrate salts. The butanol extract was then reduced to dryness in vacuo and the resulting yellow-brown residue was dissolved, by sonicating for $20 \mathrm{~min}$, in a solution of dimethylformamide (DMF, $10 \mathrm{~cm}^{3}$ ) plus citric acid $(10 \mathrm{mg})$. This extract was then crudely purified by injecting samples (1 $\mathrm{cm}^{3}$ ) onto a semi-preparative HPLC reverse phase C18 column using the mobile phase described previously (73:27, methanol:citrate buffer) and detecting at $\lambda 405$ nm. The amphotericin B peak was collected and discarded whilst the remaining solute that was collected was and concentrated in vacuo, before being redissolved by sonicating in DMF ( $5 \mathrm{~cm}^{3}$ with $5 \mathrm{mg}$ citric acid).

In order to get an indication of the content of this extract, an analytical reverse-phase HPLC with a diode array facility(carried out at SmithKline Beecham, Brockham Park) was taken of it with detection at $\lambda 305 \mathrm{~nm}$ (to detect any tetraenes) and at $\lambda$ $405 \mathrm{~nm}$ (to detect any heptaenes). The conditions used for these injections $(10 \mu \mathrm{l})$ utilised a different solvent system (from the crude separation used above) to ensure any peaks present would be sufficiently spread out, so as to identify individual peaks. The solvent program involved a gradual increase in concentration of mobile phase A in mobile phase $\mathrm{B}(0-100 \%$ over $30 \mathrm{~min})$, followed by $20 \mathrm{~min}$ isocratic in mobile 
phase B [mobile phase A: 40:60, methanol-acetonitrile (3:2) : $\mathrm{NH}_{4} \mathrm{OAc}$ (aq., 0.001 mol dm ${ }^{-3}$ ); mobile phase B: 80:20, methanol-acetonitrile (3:2) : $\mathrm{NH}_{4} \mathrm{OAc}$ (aq., 0.001 mol dm${ }^{-3}$. The results of these injections can be seen in Figs. 3.21 and 3.22. At a glance, the complex compositions of the solutions can be noted, with the peaks in the HPLC traces detected either at $\lambda 305$ or $405 \mathrm{~nm}$ indicating the presence of many polyenes. The UV spectra of certain peaks can then be measured by the use of the diode array detector. At the wavelength of $305 \mathrm{~nm}$, the indicated peaks (black spots) are all determined to be tetraenes, with the characteristic UV spectra clearly evident in Fig. 3.21(b). At the wavelength of $405 \mathrm{~nm}$, the UV spectra of the indicated peaks can be seen in Fig $3.22(b)$. However, only the major peak, assigned to be amphotericin B running at a retention time of 56 min [Fig 3.21(a) peak 3], exhibits exclusively a characteristic heptaene spectrum. The other two selected peaks also indicate the presence of some additional tetraene, along with the characteristic heptaene spectrum. However, this inference may be incorrect, as actually these peaks may be running together with some of the tetraene peaks and therefore these samples may be inhomogenous (Fig. 3.23; in this figure it must be noted that the levels of the heptaene peaks are much reduced in relation to the tetraenes but the scale has been altered for ease of comparison).

From these two initial HPLC traces therefore, the complexity of the extracts is apparent. If any of the peaks detected could be extracted in sufficient quantities, they may be identified as corresponding to an intermediate in the biosynthetic pathway of amphotericin B, and thus provide more evidence for the proposed mode of assembly. 


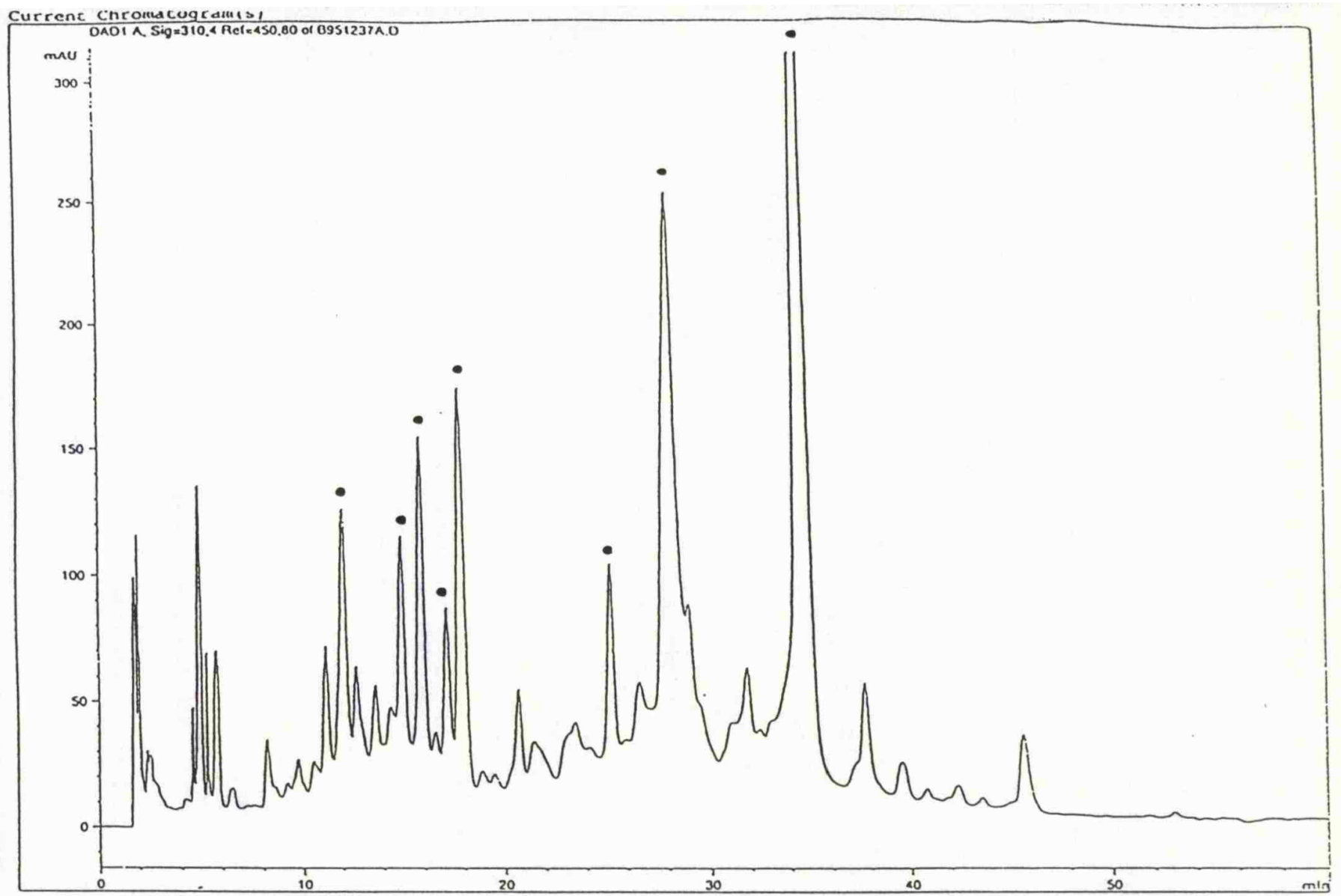

(b)

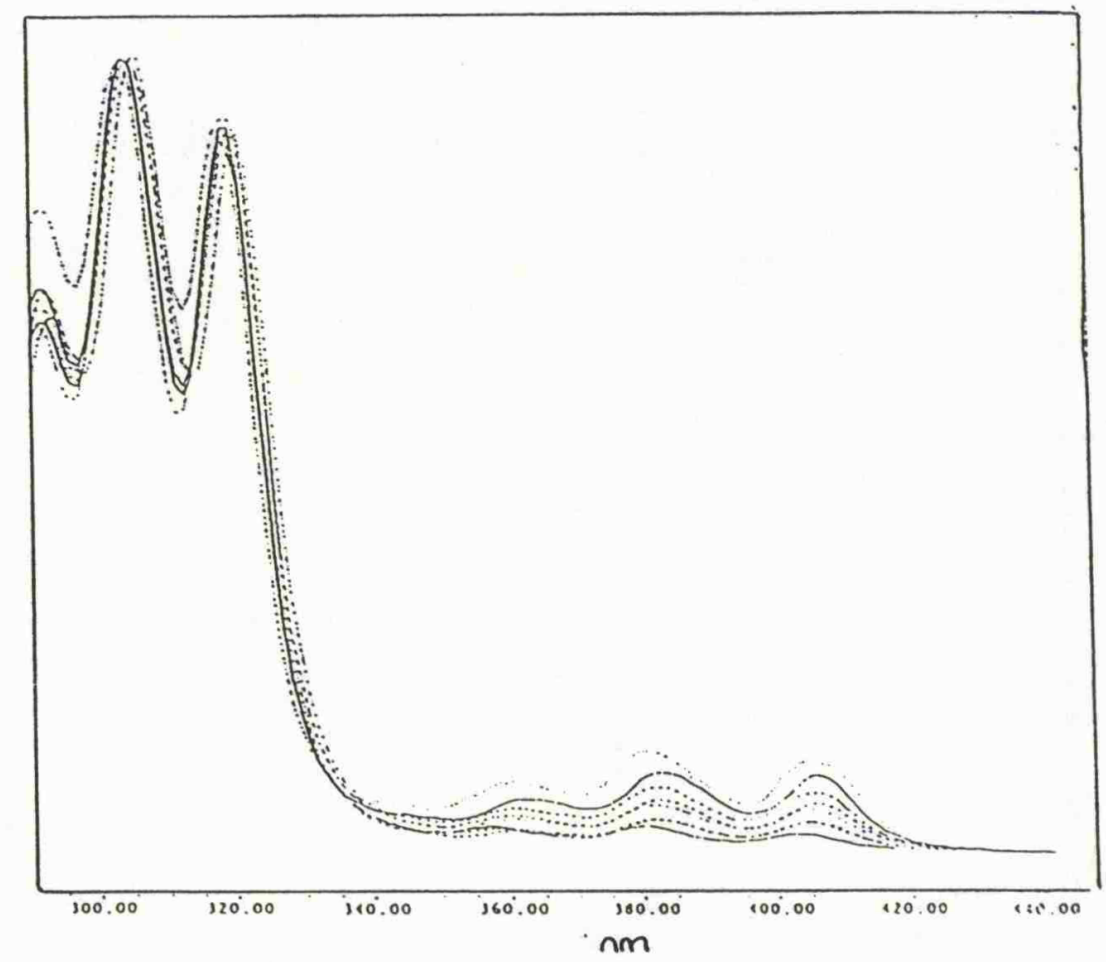

Fig. 3.21 (a) HPLC trace of the hot butanol extract detecting at $\lambda 305 \mathrm{~nm}$ and (b) UV spectrum of selected peaks (amphotericin A peak, retention time $35 \mathrm{~min}$ ) 


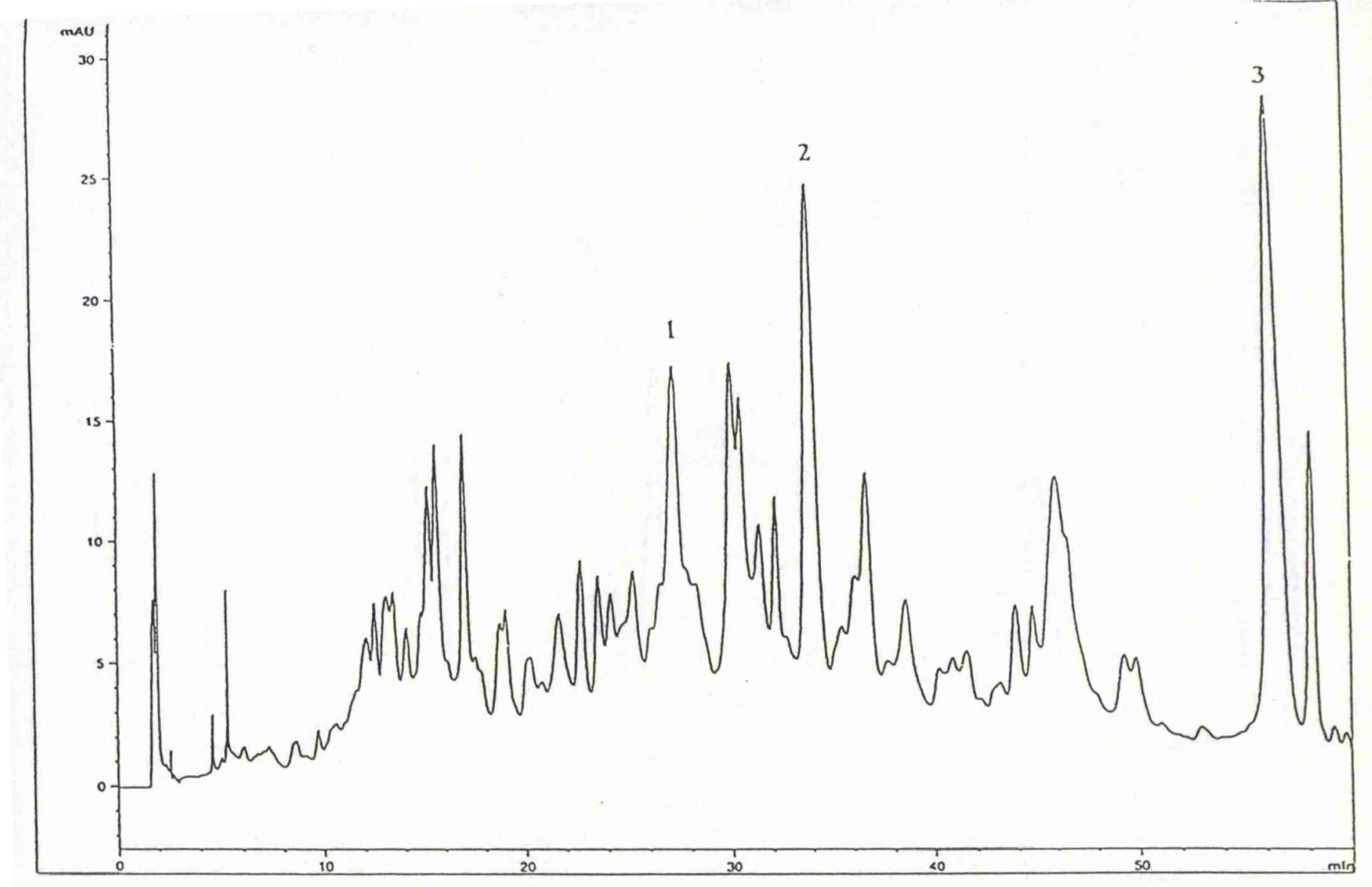

(b)

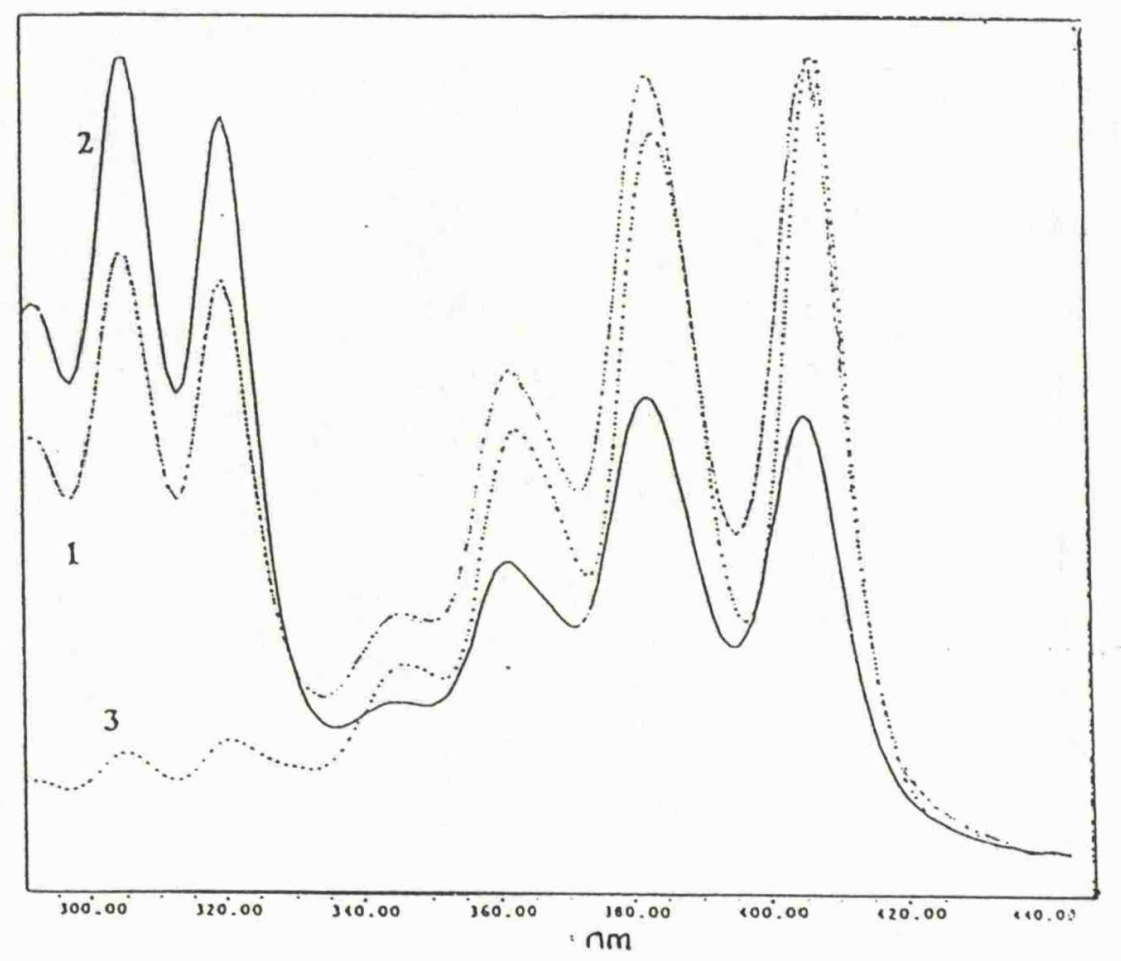

Fig. $3.22(a)$ HPLC trace of the hot butanol extract detecting at $\lambda 405 \mathrm{~mm}$ and

(b) UV spectrum of selected peaks (amphotericin B peak, retention time $56 \mathrm{~min}$ ) 


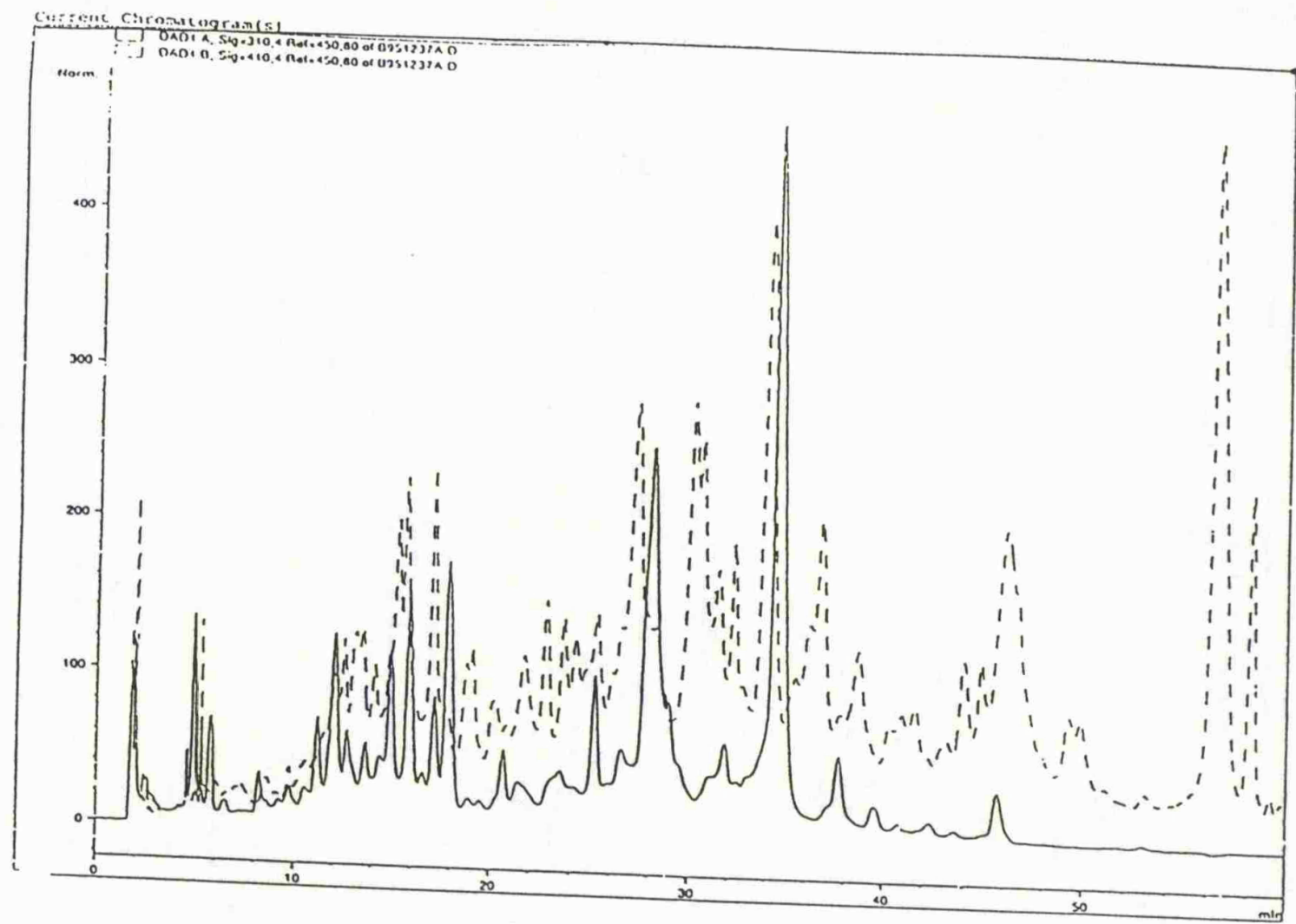

Fig. 3.23 Comparison of the traces detecting at $\lambda 305(-)$ and $405 \mathrm{~nm}(---)$

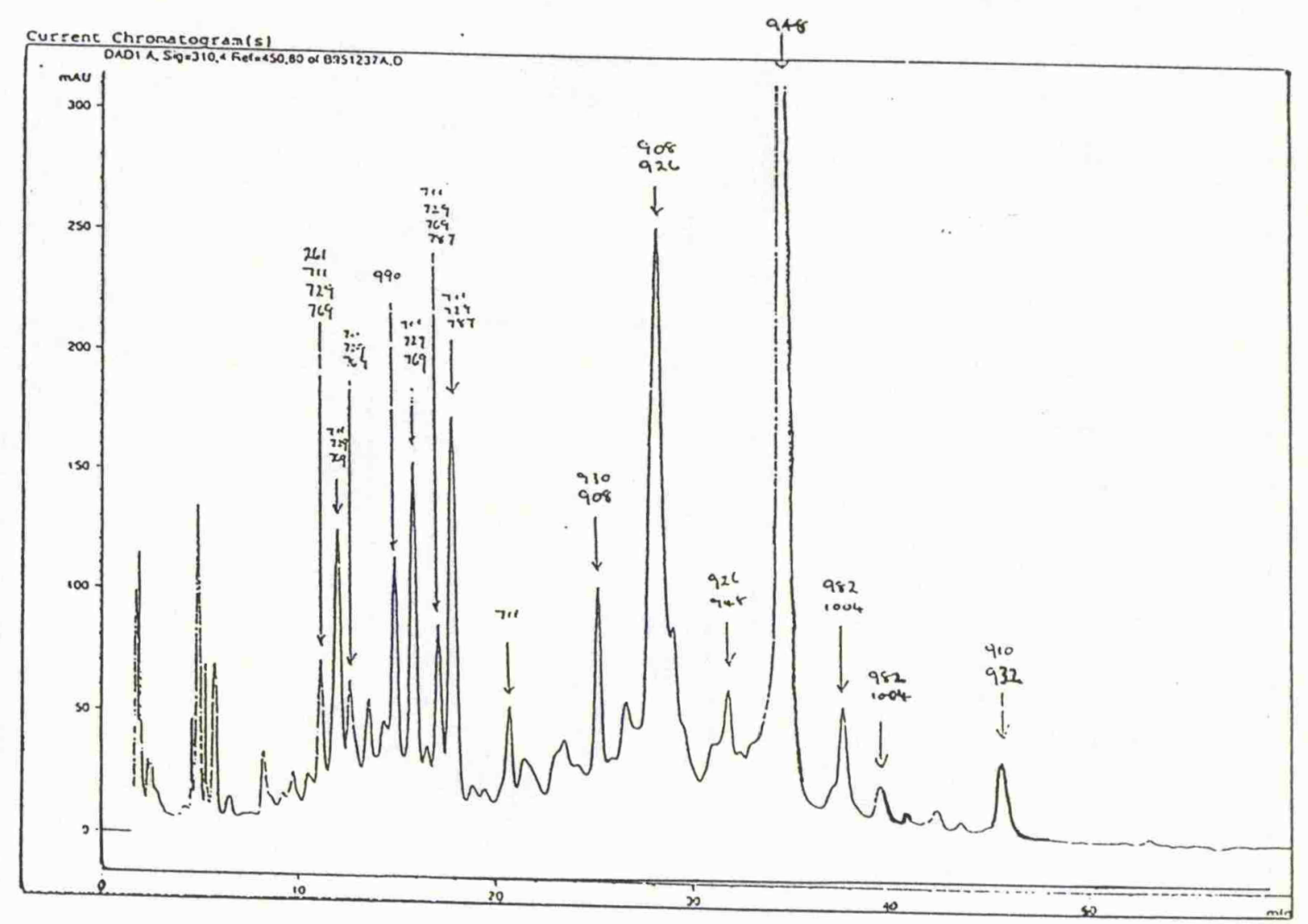

Fig. 3.24 LCMS of crude extract detecting at $\lambda 305 \mathrm{~mm}$.

with the detected mass ions for the indicated peaks 
In order to gain a further insight into the composition of the butanol extract, a sample of the DMF-citric acid solution was analysed by liquid chromatography mass spectrometry (LCMS) under the same gradient conditions as described for the analytical HPLC injection above. In Fig. 3.24 the LCMS spectrum with detection at $\lambda 305 \mathrm{~nm}$ can be seen with the calculated mass ions for the indicated peaks.

From Figs. 3.21 and 3.22, it can be seen that the levels of the tetraenes is greater than that of the heptaenes and so investigations were undertaken to isolate and purify one or more of the tetraene peaks. In order to get pure samples, the mobile phase of the HPLC was adjusted in order to further improve the separation of the peaks. The most satisfactory mobile phase was only a slight variation on the one used previously, with the solvent program having a gradual increase in concentration of mobile phase $A$ in mobile phase B (0-100\%) over 60 min. Samples of the extract (1 $\mathrm{cm}^{3}$ ) were then injected onto a semi-preparative reverse phase C18 HPLC column and $5 \mathrm{~cm}^{3}$ fractions collected. This process was then repeated four times and the comparable $5 \mathrm{~cm}^{3}$ fractions were pooled. In order to gain an indication of the contents of each fraction, a sample of each was injected onto the analytical system under isocratic conditions (40:60, mobile phase A:mobile phase $B$ ) and detecting at $\lambda$ $305 \mathrm{~nm}$. The fractions that contained interesting results can be seen in Fig. 3.25. This process was one way of purifying the concentrated extract from which certain fractions could then be selected for further purification. 
6

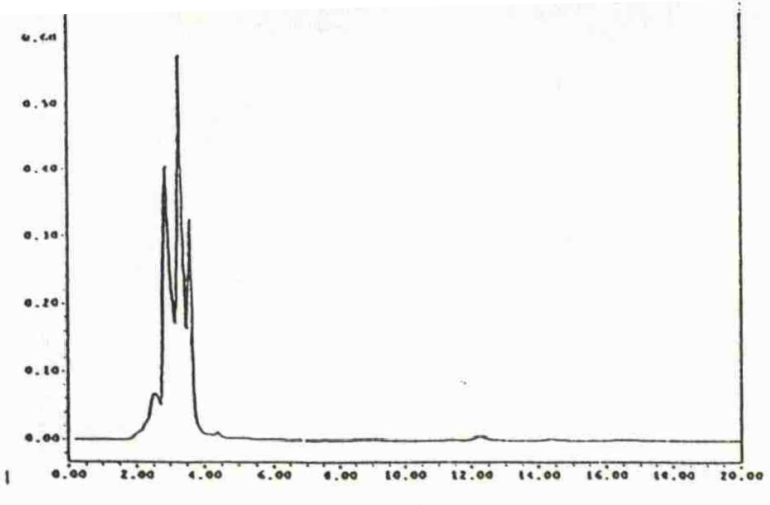

7
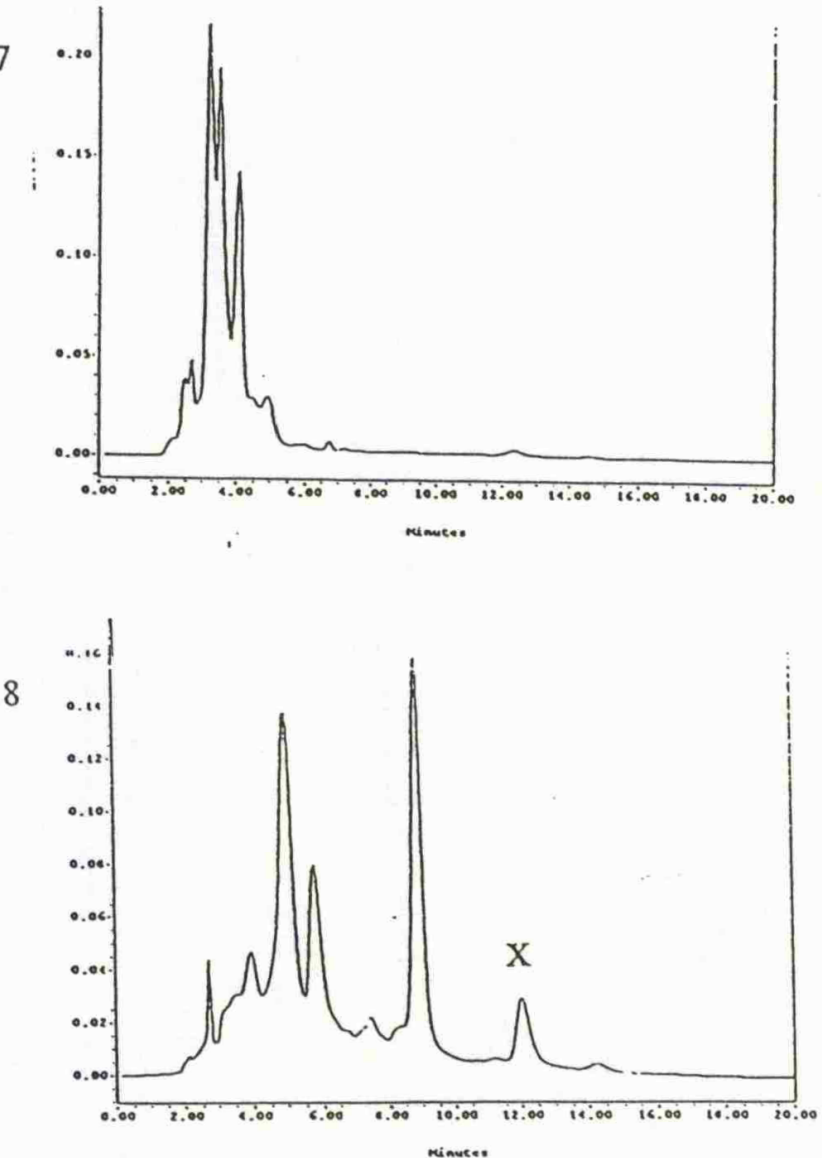

9

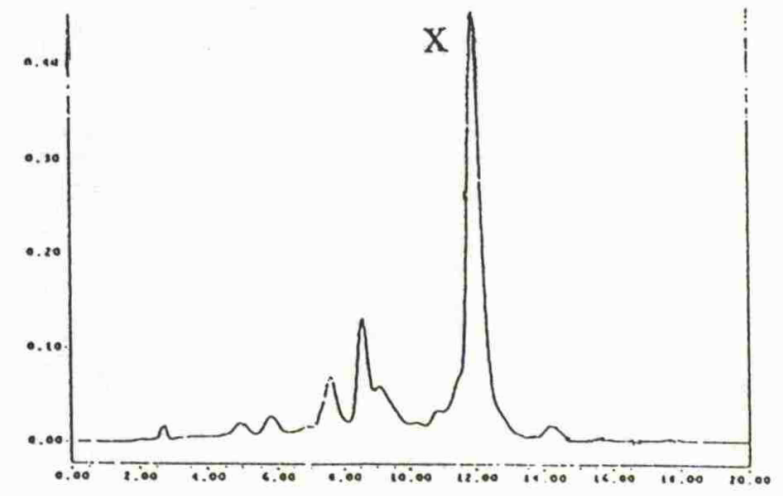

$10^{*}$
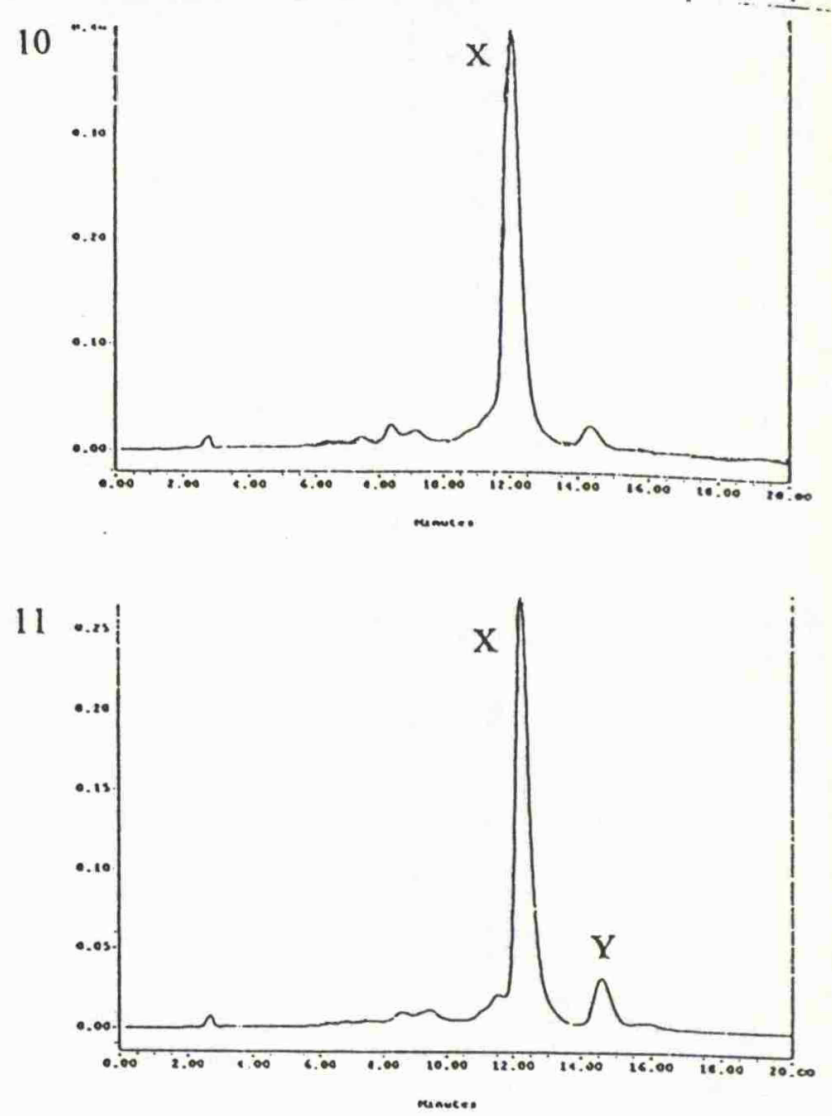

12

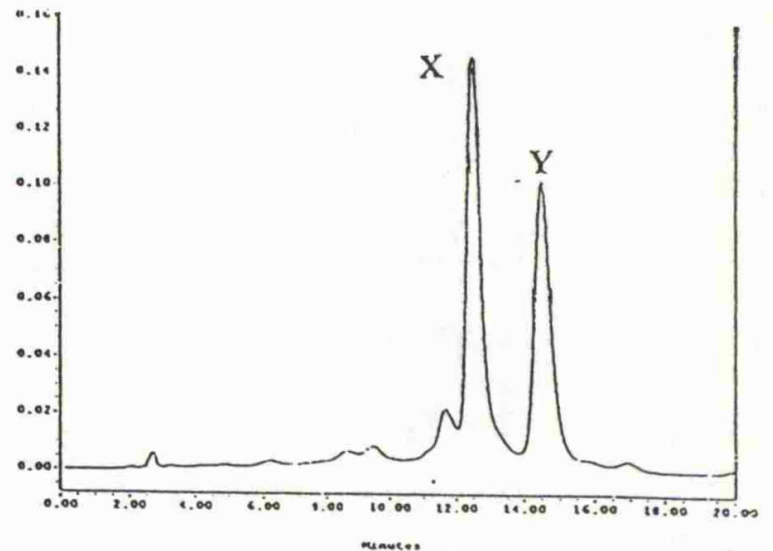

13

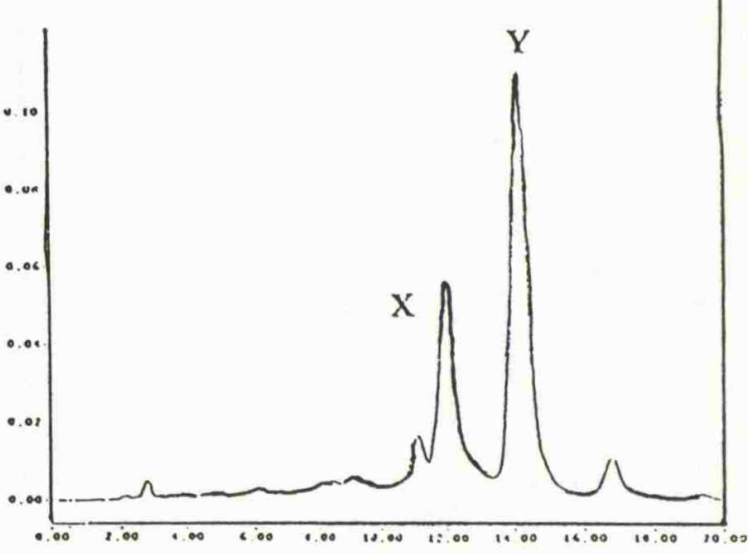

Fig. 3.25 Analytical HPLC traces detecting at $\lambda 305 \mathrm{~nm}$ under isocratic conditions of fractions 6-13 collected from the preparative HPLC 
It was apparent that fraction 10 appeared to be a single peak $\mathrm{X}$ and so this fraction was concentrated in vacuo. The resulting pale yellow residue was then redissolved in ca. $4 \mathrm{~cm}^{3}$ methanol and loaded onto an HP20 adsorption column in order to de-salt the sample. The resulting white solid $(30 \mathrm{mg})$ was then analysed by LCMS and ${ }^{1} \mathrm{H}$ NMR. The resulting LCMS spectrum can be seen in Fig. 3.26 along with the detected mass ions observed. Obviously the sample was not $100 \%$ pure, however the major peak having a molecular weight of 926 may be assigned to amphotericin A, which was confirmed by the ${ }^{1} \mathrm{H}$ NMR. ${ }^{150}$ This result is not surprising, as amphotericin A would be expected to be the major tetraene present in the fermentation broth.

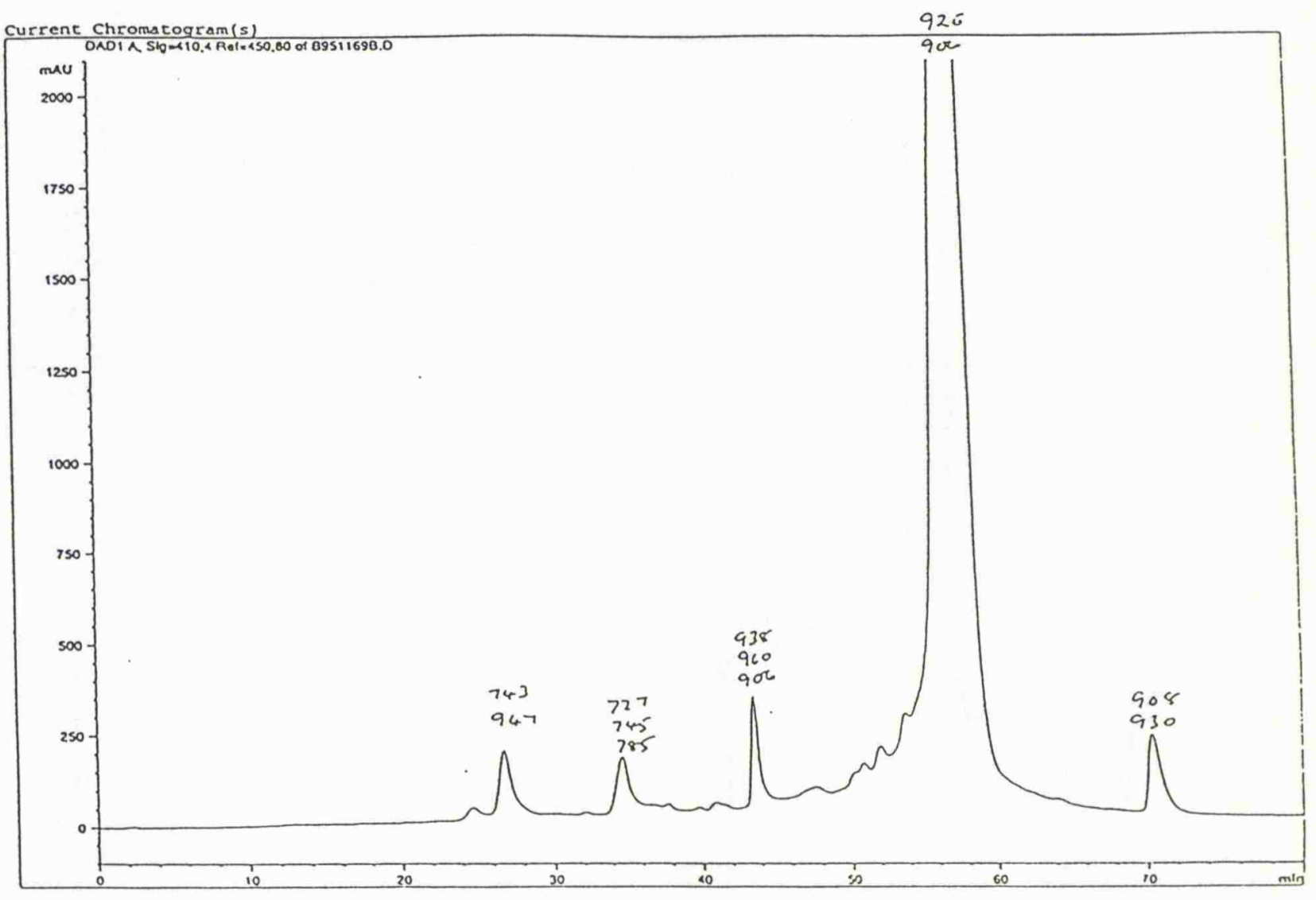

Fig. 3.26 Result for the LCMS of fraction 10 collected from the preparative HPLC 
What would be of greater interest would be to isolate one of the minor peaks that was present in the extract. Thus, the isolation of peak Y (Fig. 3.25) with a retention time of $c a$. 14.4 min was attempted, with the fractions that contained peak Y being pooled and reinjected onto the preparative HPLC. After each injection the specific peak was collected and the resulting fractions were pooled and concentrated to give a sample which by HPLC appeared fairly pure (Fig. 3.27). After de-salting the sample by running the sample through an HP20 adsorption column, the resulting pale yellow solid (4 mg) was sent for NMR analysis, but unfortunately a satisfactory spectrum was not obtained. If there had been enough time, further samples of the extract

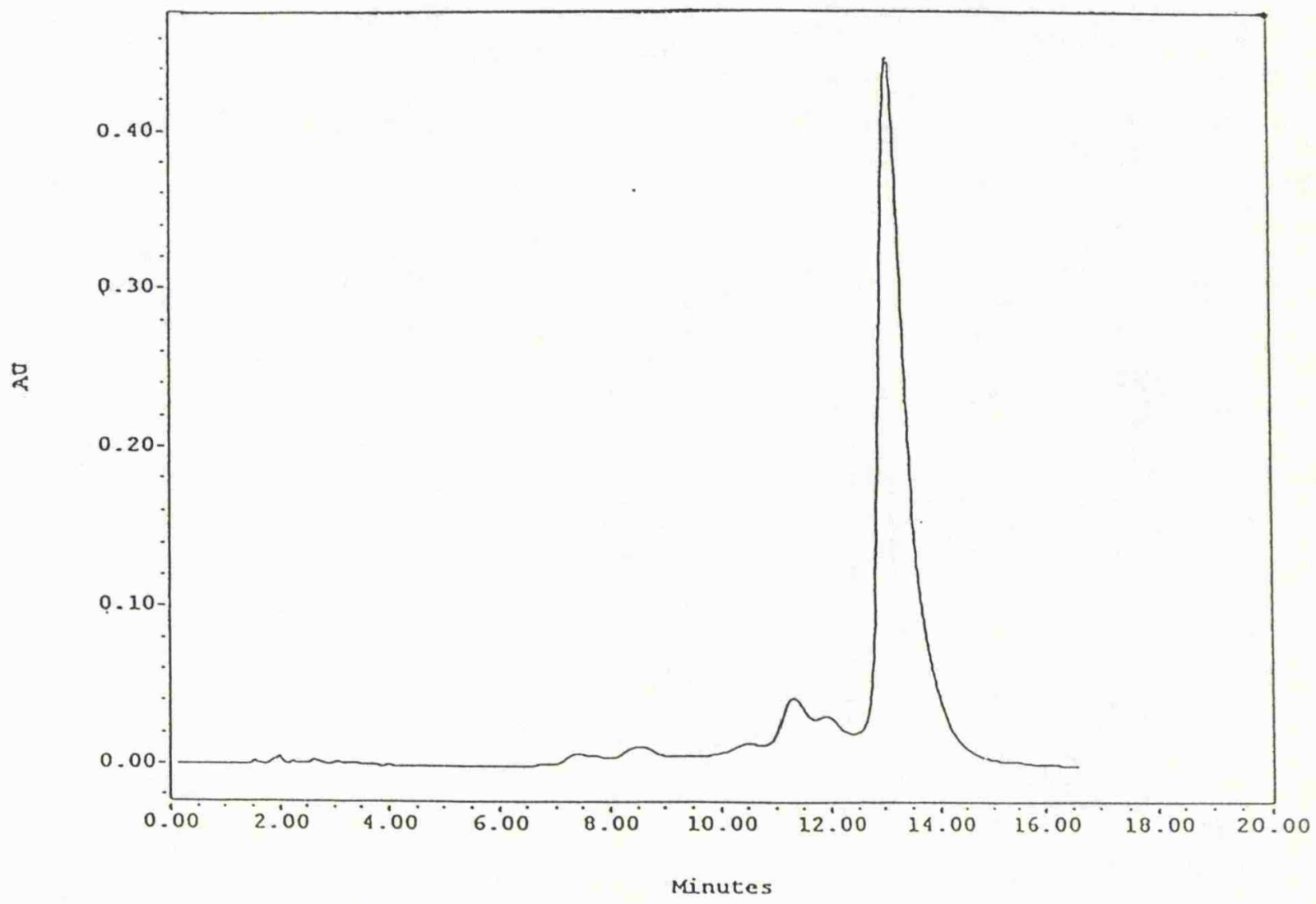

Fig. 3.27 Analytical HPLC trace of peak Y 
would have been injected in order to isolate a larger quantity of the unknown tetraene. In order to gain more information about the other peaks present, fraction 9 (Fig. 3.25) from the (pooled) preparative HPLC injections of the crude extract was concentrated in vacuo and de-salted by running through an HP20 adsorption column to give a pale yellow residue which was of insufficient quantity for an NMR spectrum. In addition, the gradient system of the analytical HPLC system was altered in order to spread the peaks out more satisfactorily (gradient: $0-100 \%$, mobile phase $\mathrm{A} \rightarrow$ mobile phase $\mathrm{B}, 80 \mathrm{~min}$, followed by $100 \% \mathrm{~B}, 10 \mathrm{~min}$; Fig. 3.28) in order to obtain run an LCMS that may give us more information about the peaks present. The results of the LCMS (Fig. 3.29) indicated six major peaks in the spectrum, the probable molecular weights of which are as indicated in Table 3.3.

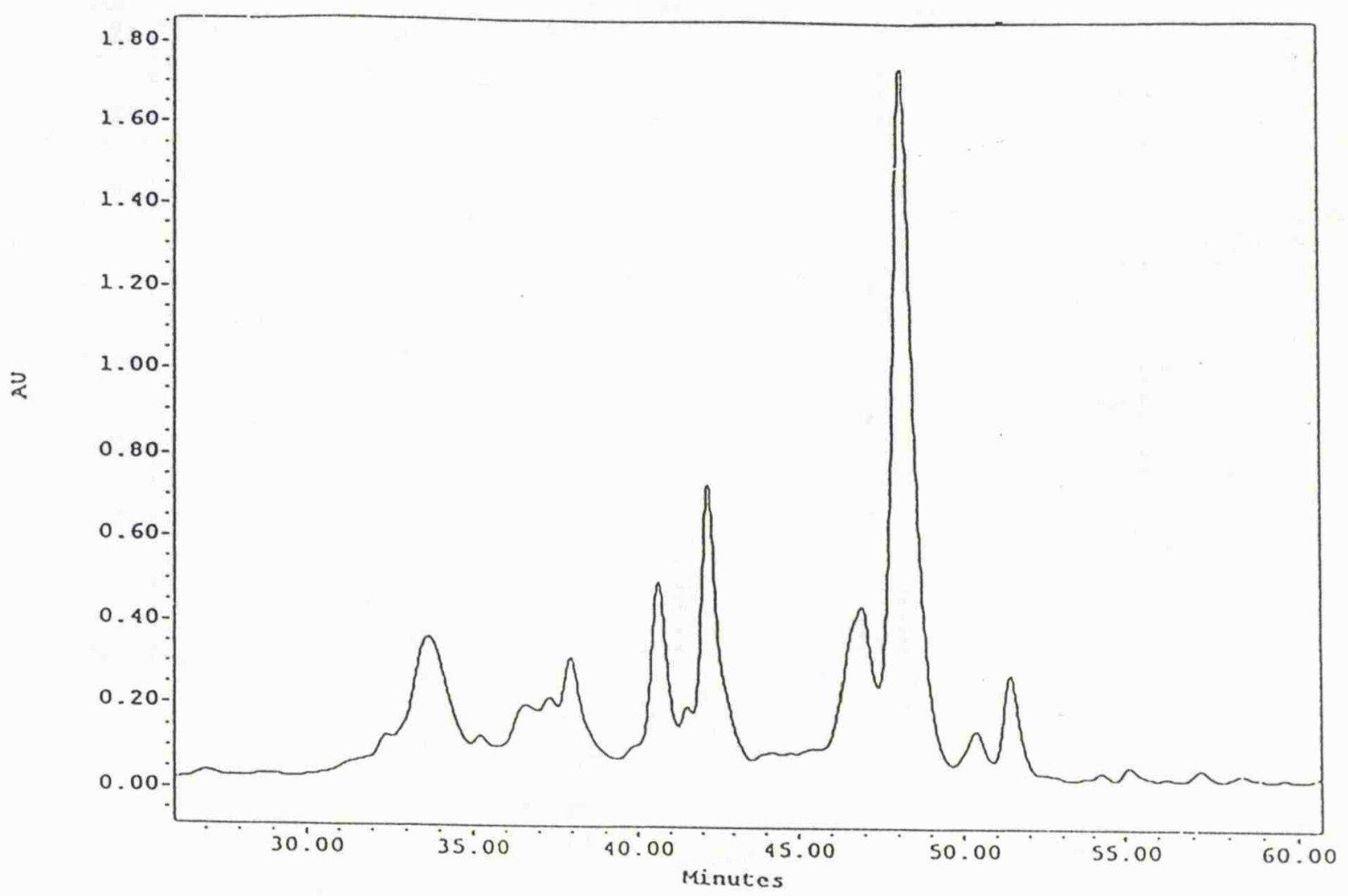

Fig. 3.28 Analytical HPLC trace under gradient conditions of fraction 9 


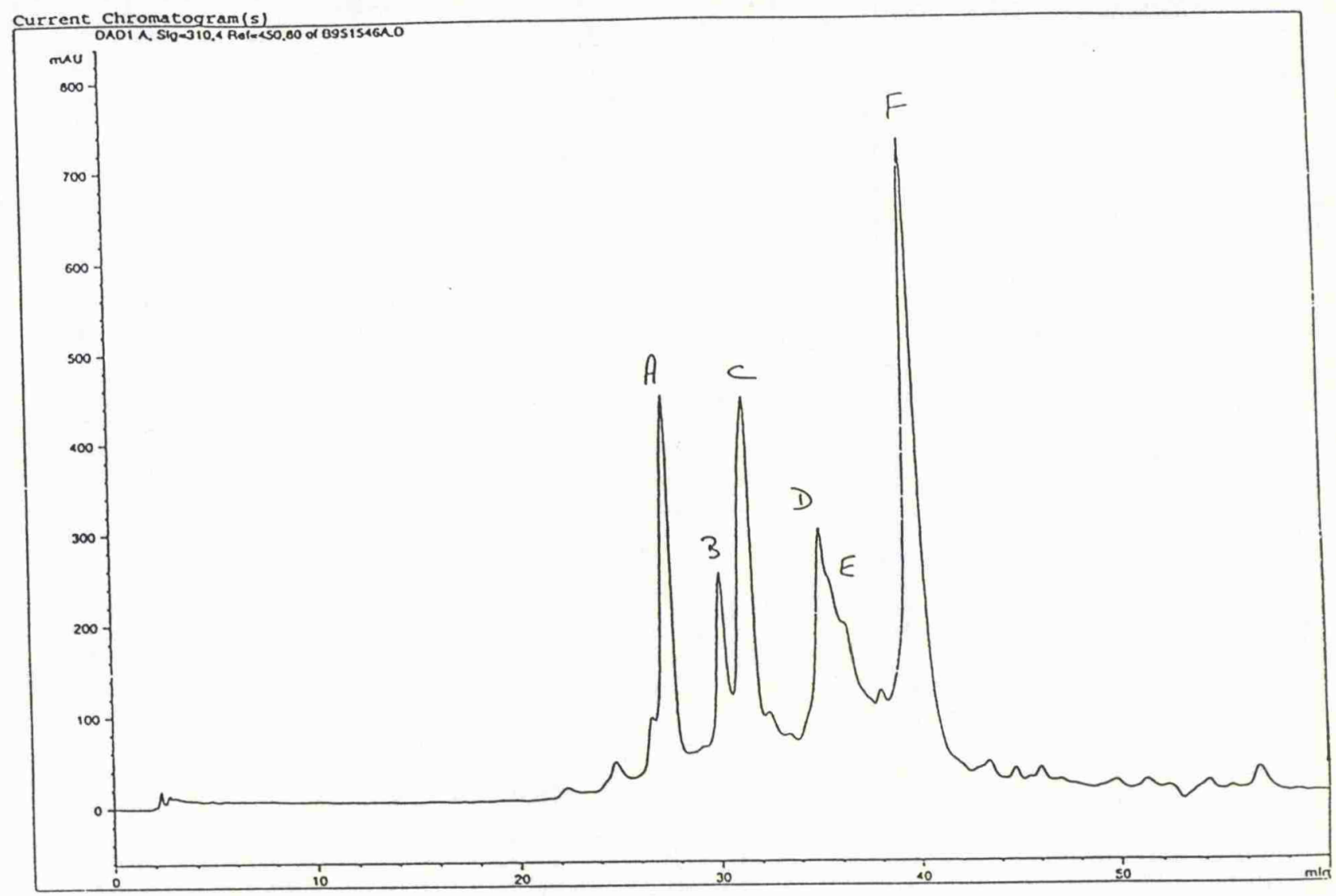

Fig. 3.29 Results from the LCMS of fraction 9

Table 3.3 The probable molecular weights (MW) of the selected peaks after a LCMS on a sample of fraction 9

\begin{tabular}{llll}
\hline Peak & $\begin{array}{l}\text { Retention } \\
\text { time/min }\end{array}$ & Ions observed & Probable MW \\
\hline & & 908,926 & $907+925$ \\
A & 27.20 & 926 & 925 \\
B & 30.10 & 908 & 907 \\
C & 31.20 & 940,926 & $939+925$ \\
D & 35.10 & 892 & 891 \\
E & 35.30 & 926 & 925 \\
F & 39.50 & & \\
\hline
\end{tabular}


The results of the LCMS of this fraction, and those of the initial overall crude extract

(Fig. 3.24), provide some information about the minor metabolites present in the fermentation broth. Possible structures can be assigned to the peaks of certain molecular weights (e.g. MW 939 may correspond to a compound that utilised propionate as a 'starter' unit instead of acetate) and if those peaks could then be isolated in sufficient quantities, further analysis could be carried out.

Although this investigation has not provided detailed structures of other metabolites in the fermentation broth, it has demonstrated the presence of a large number of heptaene and tetraenes in the extracts at low levels. Unfortunately, the complexity of the extracts and the similar retention times of many of the components precluded the identification of any one compound. The nature of these minor metabolites as possible intermediates in the biosynthetic pathway of amphotericin B warrants further investigation. 


\section{$\mathbb{C}$ 咠aperer 4}

\section{Labelliling studies}

\subsection{Hntroduction}

The main aim of this study was to investigate the biosynthesis of amphotericin B and to ultimately provide some evidence for the processes by which this metabolite is produced in nature. Whilst most classes of polyketides have been extensively investigated by the incorporation of isotopically labelled compounds, few biosynthetic investigations have been undertaken on the polyenes. The reason for this is, in part, due to the difficulty in isolating many of these metabolites. One polyene that has been investigated extensively has been the antifungal agent fungichromin 46. The origin of the carbon skeleton has been elucidated by the incorporation labelled acetate, propionate and octanoate (Fig. 4.1) with intact incorporation being detected by NMR techniques. ${ }^{29,62}$

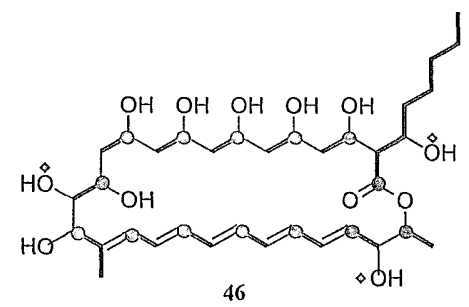

Fig. 4.1 Structural skeleton of fungichromin, with the indicated acetate, propionate and octanoate sub-units; ${ }^{\circ 13} \mathrm{C},{ }^{18} \mathrm{O}$ from ${ }^{18} \mathrm{O}_{2}$ 
Although amphotericin B is an extremely important, if highly toxic, antibiotic, it has been the target of very few biosynthetic investigations. The only feeding experiments reported to date involving amphotericin B employed the addition of [2${ }^{14} \mathrm{C}$ lacetate to prepare radioactively labelled amphotericin B for clinical studies. ${ }^{70-72}$ The use of stable isotopes in order to provide information about the biosynthetic origin of amphotericin B was therefore novel for this metabolite and a variety of feedings were undertaken in an attempt to gain an understanding of the processes involved.

A possible biosynthetic pathway for amphotericin $B$ has been proposed, in which the macrocyclic ring is built up of acetate and propionate sub-units through the processive pathway (see Chapter 1, Fig. 1.23). To obtain some information about the origin of the carbon chain and provide support for this proposed pathway, variously labelled carbon-13 acetate-derived compounds were initially fed to cultures of the microorganism (S. nodosus) and analysis of the incorporation was carried out by NMR. To further investigate the mode of assembly (processive or non-processive pathway) a putative labelled diketide intermediate was synthesised and administered to cultures of $S$. nodosus, with any incorporation to be detected by electrospray mass spectrometry, as discussed in the previous chapters. 


\subsection{Acetate feedings}

It was necessary to develop suitable protocols for the growth of cultures of $S$. nodosus, along with the techniques required for the purification and analysis of amphotericin B, before the addition of any expensive or synthesised labelled putative precursors. Therefore preliminary experiments were carried out prior to any labelled compounds being fed to the culture medium. The exact protocol as to how and when to feed the labelled compounds had to be determined.

Previous studies have employed a variety of methods of administering the labels. As discussed in Chapter 3, some investigations have employed the use of replacement media during the growth of the microorganism. This approach can be modified by the use of replacement media in which the sodium salt of $\left[2-{ }^{13} \mathrm{C}\right]$ acetate is present as the sole carbon source. ${ }^{156}$ This methodology is advantageous because as the labelled acetate is the only carbon source, the chances of it being incorporated by the microorganism are greatly increased. In our studies, this approach was investigated with acetate present as the sole carbon source, along with some experiments having acetate and glucose present (in a ratio of 1:1), however the growth of $S$. nodosus and production of amphotericin B under both these conditions were much reduced.

Other approaches have involved the use of a pulsed feeding technique, in which aliquots of the labelled acetate are added to the culture broth at specified times either as the free acid or sodium salt, and in certain cases diluted with unlabelled 
material. ${ }^{155-158} \quad\left[1,2-{ }^{13} \mathrm{C}_{2}\right]$ Acetate will normally become assimilated into the metabolite after dilution with the microorganism's own unlabelled acetate which is mainly derived from glucose or fatty acid oxidation. It is statistically unlikely ${ }^{159}$ that labelled acetate units will become incorporated consecutively in a chain during biosynthesis; however if this were to happen with a doubly labelled compound as a result of very high incorporation, the resulting NMR spectrum of the product would be very complicated, and in order to avoid this, additional unlabelled acetate can be fed simultaneously to the culture.

However, there is usually a sufficient natural pool of unlabelled material present in the culture broth so that if coupled signals are detected in the NMR spectrum, they are an indication that the doubly labelled acetate has been incorporated intact. The nuclei of adjacent labelled carbon atoms in the intact acetate are part of the same biosynthetic unit and have couplings that produce doublets superimposed on the natural abundance singlets (detection of intra-unit coupling). If a doubly labelled acetate unit gets fragmented however, then only an enhanced singlet will be present in the NMR spectrum for each incorporated labelled carbon. Administration of a combination of different singly labelled compounds may also be employed, with incorporation of the single labels being detected by specific enhancements of the corresponding signals in the NMR spectra. If however there is a very high incorporation, inter-unit couplings may be detected between labelled carbons of adjacent acetate units. 
One of the main reasons that so few investigations have been reported on polyene antibiotics is perhaps due to the complexity of their NMR spectra. The assignment of certain peaks in the NMR is very difficult for such polyenes, including amphotericin $B$, because of the presence of repetitive identical structural fragments; however the majority of peaks in the ${ }^{1} \mathrm{H}$ and ${ }^{13} \mathrm{C}$ NMR of amphotericin $\mathrm{B}$ have been successfully assigned ${ }^{150-153}$ and shall be the point of reference for this study. In our study, investigations involving variously labelled compounds were carried out in an attempt to gain information about the biosynthesis of the skeleton of amphotericin B.

Preliminary studies, prior to the feeding of labelled compounds, involved the feeding of unlabelled acetate to the fermentation medium to monitor any effect this might have on the production of amphotericin B. Pulsed feeding techniques were applied in this, and subsequent experiments, in which specified aliquots of compound were added at certain times over the production time-course. The specified amounts of compound were dissolved in samples of sterilised water prior to addition to the medium. The protocol for the fermentation procedure in FCA media was formulated as described in the previous chapter. Unlabelled acetate $(100 \mathrm{mg}$ portions in sterilised $250 \mu \mathrm{l}$ of water per feeding) was added to a $500 \mathrm{~cm}^{3}$ shake flask, containing $60 \mathrm{~cm}^{3}$ of whole broth at 24,48 and $70 \mathrm{~h}$ after inoculation. Simultaneously, a flask of whole broth was grown with no added acetate, to act as a control. The assay of amphotericin B production in both flasks can be seen in Fig. 4.2, with production levels appearing similar in both flasks. Thus, the addition of acetate had minimal adverse effects on the production of metabolite. 


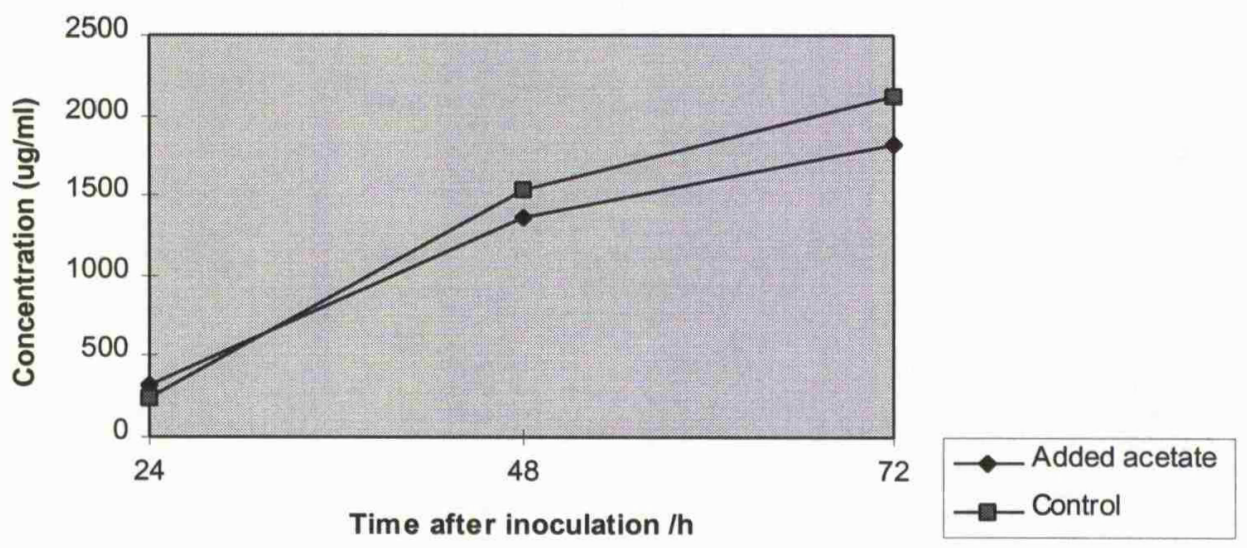

Fig. 4.2 Preliminary experiment for acetate feeding to compare production of $\mathrm{AmB}$ with and without addition of unlabelled acetate to the fermentation flask

\subsection{1 $\left[1,2-{ }^{13} \mathrm{C}_{2}\right]$ Acetate feeding}

The doubly labelled acetate was administered to the culture broth, following the procedure described in the previous chapter (with $60 \mathrm{~cm}^{3}$ of FCA media per $500 \mathrm{~cm}^{3}$ shake flask, unless otherwise stated). $\left[1,2-{ }^{13} \mathrm{C}_{2}\right]$ Acetate was divided between four flasks and pulse-fed to the whole broth over three days. As production of the metabolite is slow over the first $24 \mathrm{~h}$ after inoculation, there was only one pulsefeeding in this time period. The aliquots of acetate $(42 \mathrm{mg}$ in $200 \mu 1$ of sterilised water per flask per feeding) were administered at $24,40,44,48,64,68$ and $70 \mathrm{~h}$ after inoculation. The production of amphotericin B was monitored by the usual UV assay (Fig. 4.3). After $72 \mathrm{~h}$, the whole broth from the four flasks was then pooled and the extraction procedure carried out as previously described with ethyl acetate and Aliquat 336. The precipitation process was monitored (UV assay, Table 4.1) to 


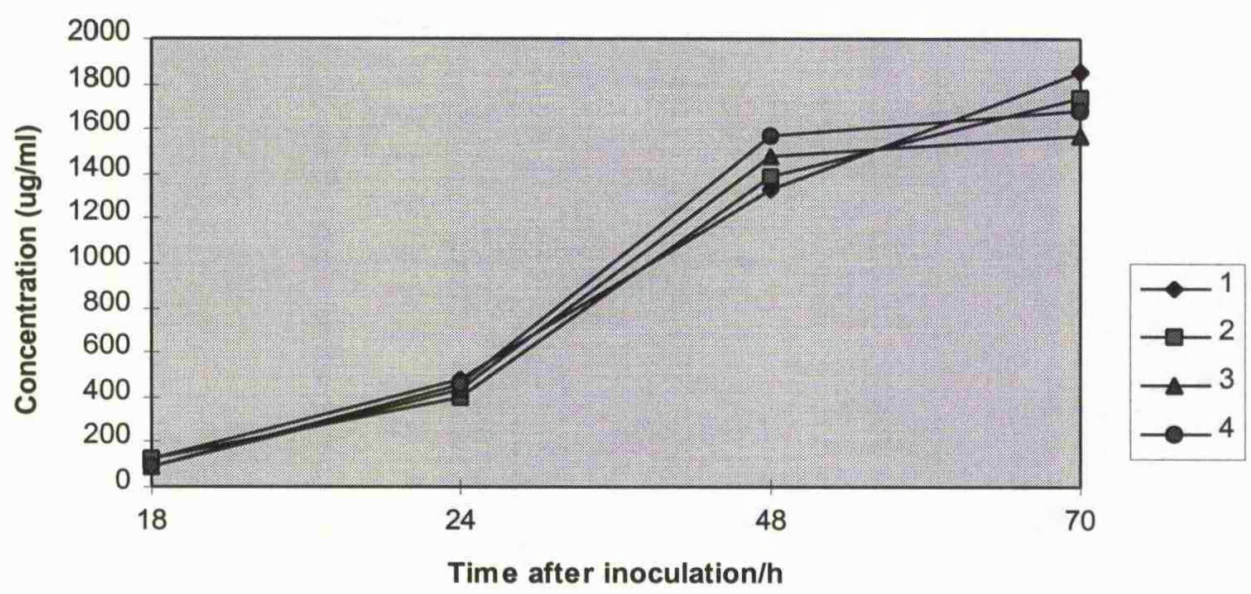

Fig. 4.3 UV assay of the fermentation broth after the addition of

$\left[1,2-{ }^{13} C_{2}\right]$ acetate to the four shake flasks $(1,2,3,4)$

Table 4.1 UV assay of the precipitation process of amphotericin B from the ethyl acetateAliquat extract of $4 \times 60 \mathrm{~cm}^{3}$ of whole broth samples following the addition of [1,2${ }^{13} \mathrm{C}_{2}$ ]acetate

\begin{tabular}{llll}
\hline Day & $\mathrm{pH}$ & Concentration $/ \mu \mathrm{g} \mathrm{ml}^{-1}$ & \\
& & Amphotericin $\mathrm{A}^{a}$ & Amphotericin $\mathrm{B}^{b}$ \\
\hline 1 & 9.7 & 2732 & 1641 \\
4 & 7 & 2941 & 291 \\
5 & 7 & 2453 & 233 \\
6 & 7 & 2500 & 241 \\
\hline
\end{tabular}

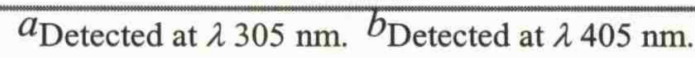


detect when all the metabolite had precipitated. This was then recovered and dried to give $300 \mathrm{mg}$ of amphotericin $\mathrm{B}$ as a yellow solid, $20 \mathrm{mg}$ of which was then dissolved in $\left[{ }^{2} \mathrm{H}_{6}\right] \mathrm{DMSO}\left(0.6 \mathrm{~cm}^{3}\right)$ and analysed by ${ }^{13} \mathrm{C}\left\{{ }^{1} \mathrm{H}\right\}$ NMR.

The peaks in the NMR were assigned according to previously published results, ${ }^{150-153}$ the details of which can be seen in Table 4.2, along with the structure of amphotericin B with the designated numbering system. For comparison, the ${ }^{13} \mathrm{C}\left\{{ }^{1} \mathrm{H}\right\}$ NMR spectrum of a standard sample of amphotericin B can be seen in Fig. 4.4. The NMR of the precipitated amphotericin B (Fig. 4.5) clearly indicates that there was successful incorporation of the labelled acetate, although not all the peaks could be accurately assigned due to the overlapping of some resonances. In the ${ }^{13} \mathrm{C}\left\{{ }^{1} \mathrm{H}\right\} \mathrm{NMR}$ spectrum of the labelled sample of amphotericin B, the doublets superimposed on the natural abundance singlets arising from couplings between intact labelled acetate units, are clearly visible. The most distinct signals are those corresponding to $\mathrm{C}-1$ (the lactone carbon; 170.4 ppm), C-13 (96.9 ppm) and C-38 (16.8 ppm; Fig. 4.5 and 4.6). What was also evident in this NMR was the lack of doublets for the signals corresponding to the carbons presumed to be derived from propionate subunits (peaks at $11.9,18.4,58.4,77.1$ and $176.8 \mathrm{ppm}$; other presumed propionate derived carbon signals are not clearly distinguishable due to overlapping resonances). From the NMR spectra it does seem however that some of these singlet peaks are enhanced, particularly those corresponding to C-39 (11.9 ppm) and C-40 (18.4 ppm). Any enhancement at the propionate positions would provide evidence for the incorporation of a fragmented acetate unit. This can occur as the labelled acetate 
Table 4.2 Assignment of the ${ }^{13} \mathrm{C}$ NMR spectra of amphotericin $\mathrm{B} 150$

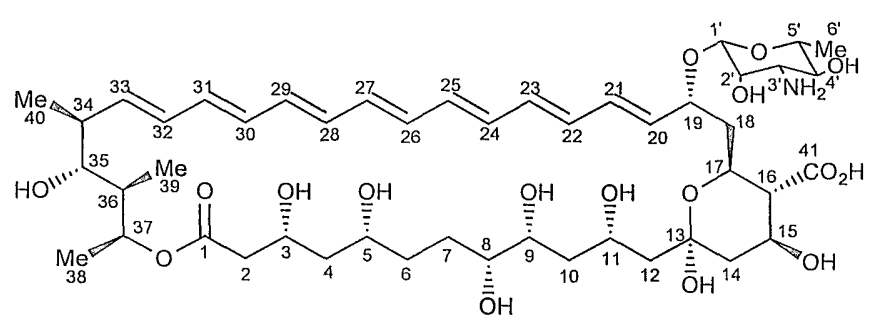

\begin{tabular}{|c|c|}
\hline Carbon shift / ppm & Carbon \\
\hline 11.9 & $\mathrm{C}-39$ \\
\hline 16.8 & $\mathrm{C}-38$ \\
\hline 17.8 & $\mathrm{C}-6^{\prime}$ \\
\hline 18.4 & $C-40$ \\
\hline 29.0 & $\mathrm{C}-7$ \\
\hline 35.0 & $\mathrm{C}-6$ \\
\hline 36.3 & $\mathrm{C}-18$ \\
\hline 39.6 & $\mathrm{C}-10$ \\
\hline 39.7 & $\mathrm{C}-36$ \\
\hline 41.9 & $\mathrm{C}-2$ \\
\hline 42.4 & $\mathrm{C}-34$ \\
\hline 44.3 & $\mathrm{C}-4$ \\
\hline 44.6 & $\mathrm{C}-14$ \\
\hline 46.3 & $\mathrm{C}-12$ \\
\hline 56.0 & $\mathrm{C}-3^{\prime}$ \\
\hline 58.4 & $\mathrm{C}-16$ \\
\hline 65.2 & C-17 \\
\hline 65.4 & $\mathrm{C}-15$ \\
\hline 66.1 & C-3 \\
\hline 67.7 & $\mathrm{C}-11$ \\
\hline 68.7 & $\mathrm{C}-2^{\prime}$ \\
\hline 69.0 & $\mathrm{C}-37$ \\
\hline 69.7 & $\mathrm{C}-5$ \\
\hline 70.5 & $\mathrm{C}-4^{\prime}$ \\
\hline 72.5 & $\mathrm{C}-5^{\prime}$ \\
\hline 73.4 & $\mathrm{C}-9$ \\
\hline 73.8 & C-8 \\
\hline 74.1 & C-19 \\
\hline 77.1 & $C-35$ \\
\hline 95.5 & $\mathrm{C}-1^{\prime}$ \\
\hline 96.9 & C-13 \\
\hline $128-136.6$ & C-20-C-33 \\
\hline 170.4 & C-1 \\
\hline 176.8 & $\mathrm{C}-41$ \\
\hline
\end{tabular}




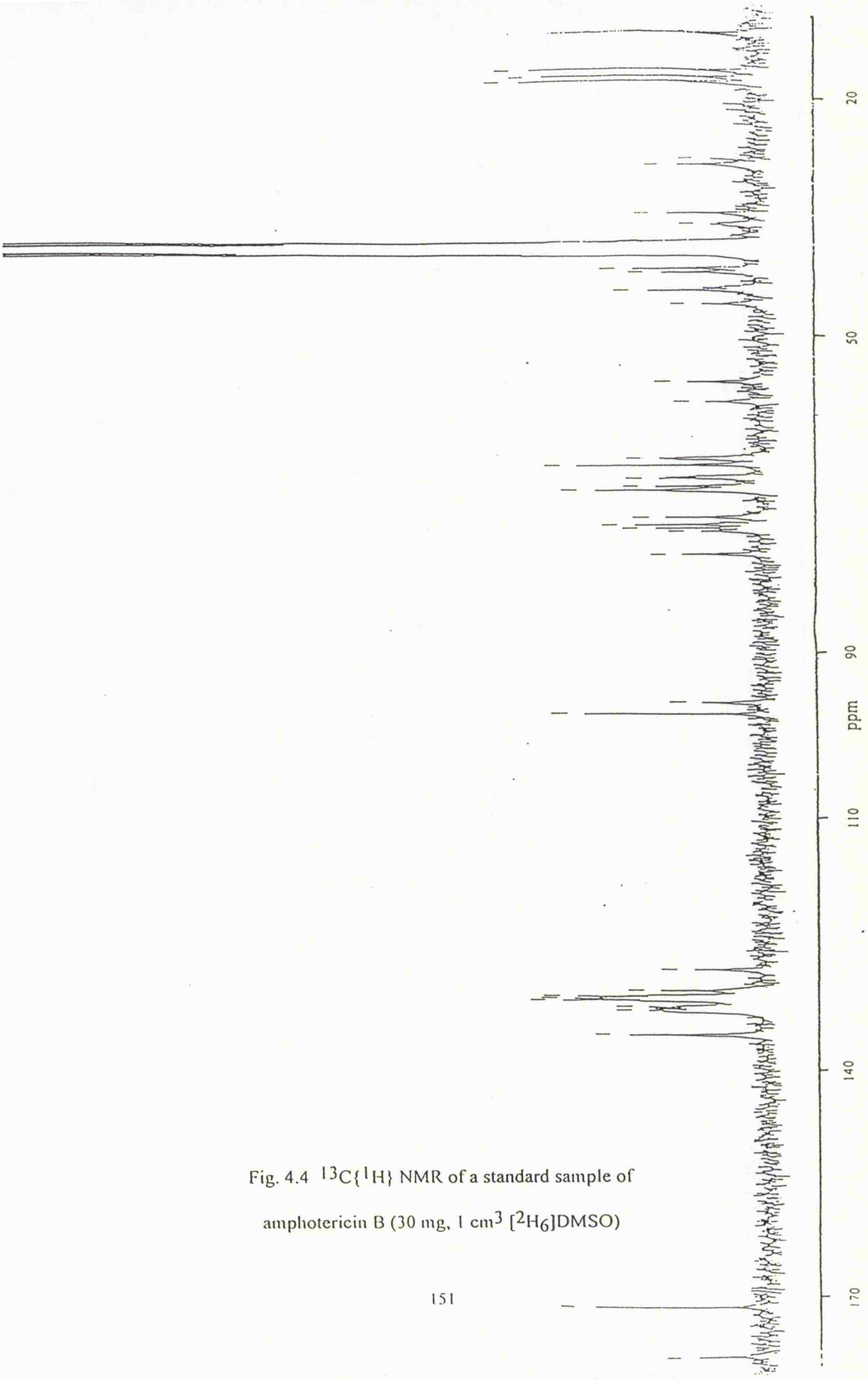



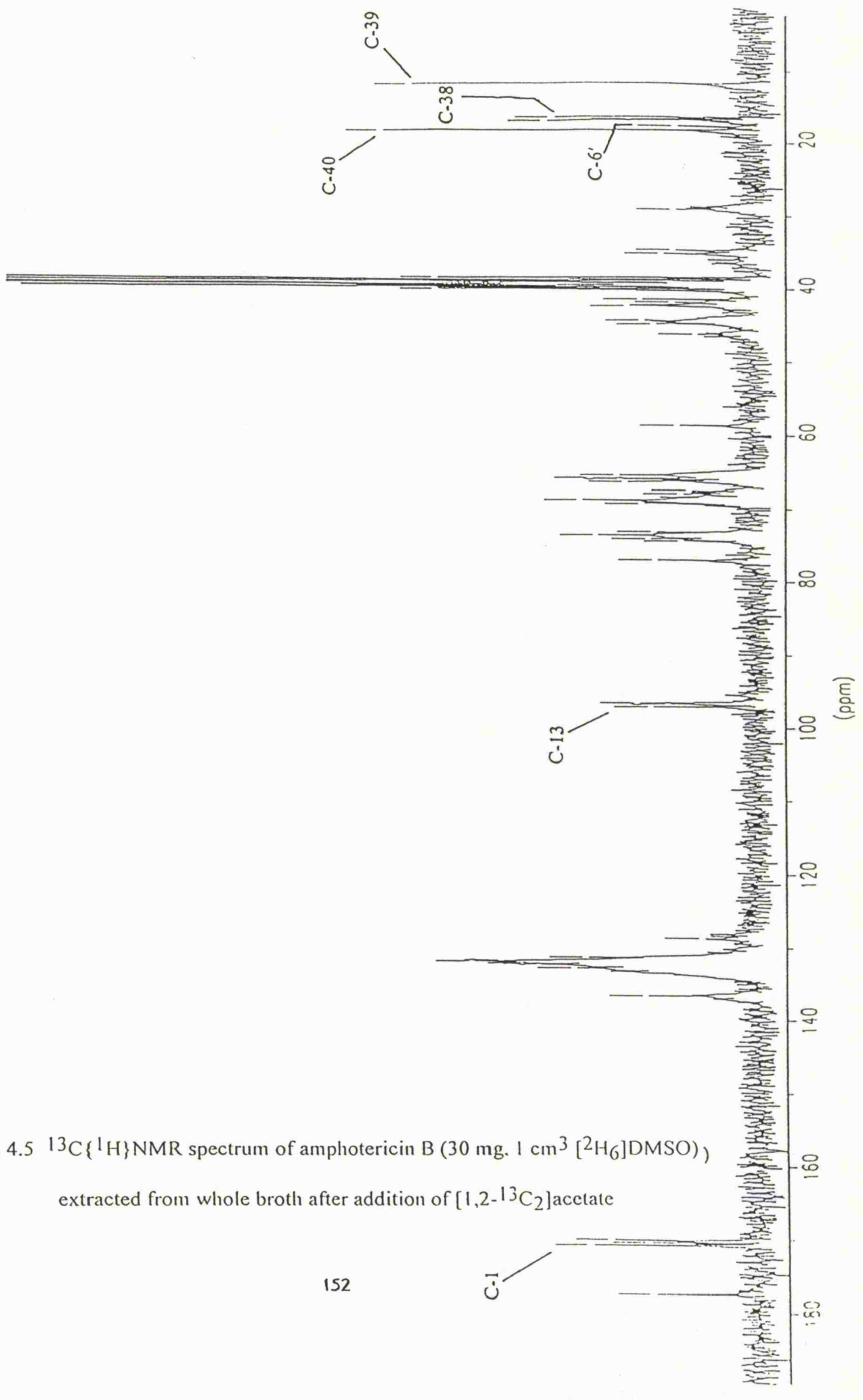

Fig. $4.5{ }^{13} \mathrm{C}\left({ }^{1} \mathrm{H}\right\} N M R$ spectrum of amphotericin $\left.\mathrm{B}\left(30 \mathrm{mg} .1 \mathrm{~cm}^{3}\left[{ }^{2} \mathrm{H}_{6}\right] \mathrm{DMSO}\right)\right)$ extracted from whole broth after addition of $\left[1,2-1^{13} \mathrm{C}_{2}\right]$ acetate 
(a)

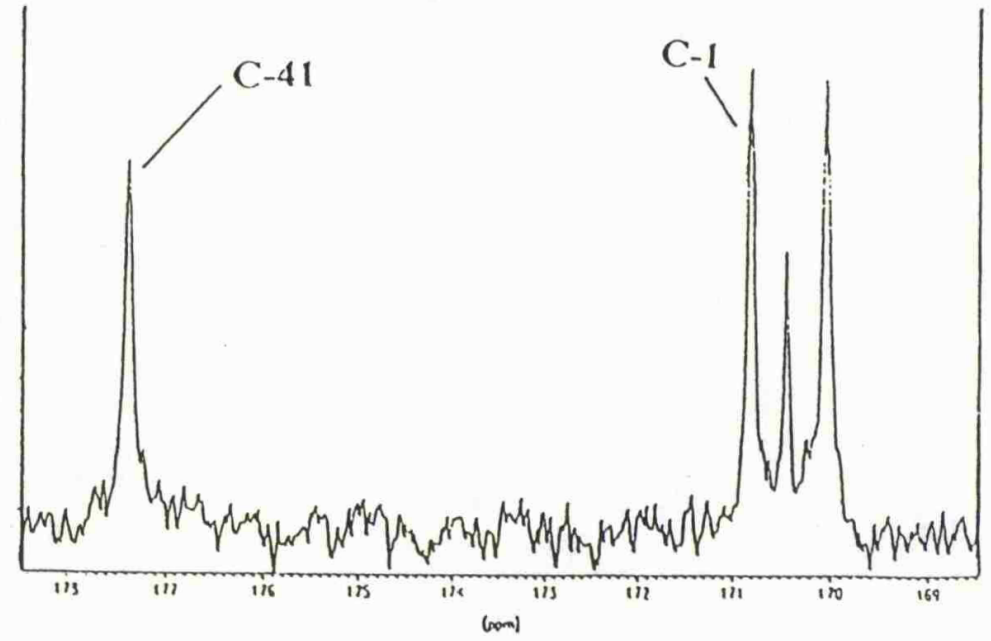

(b)

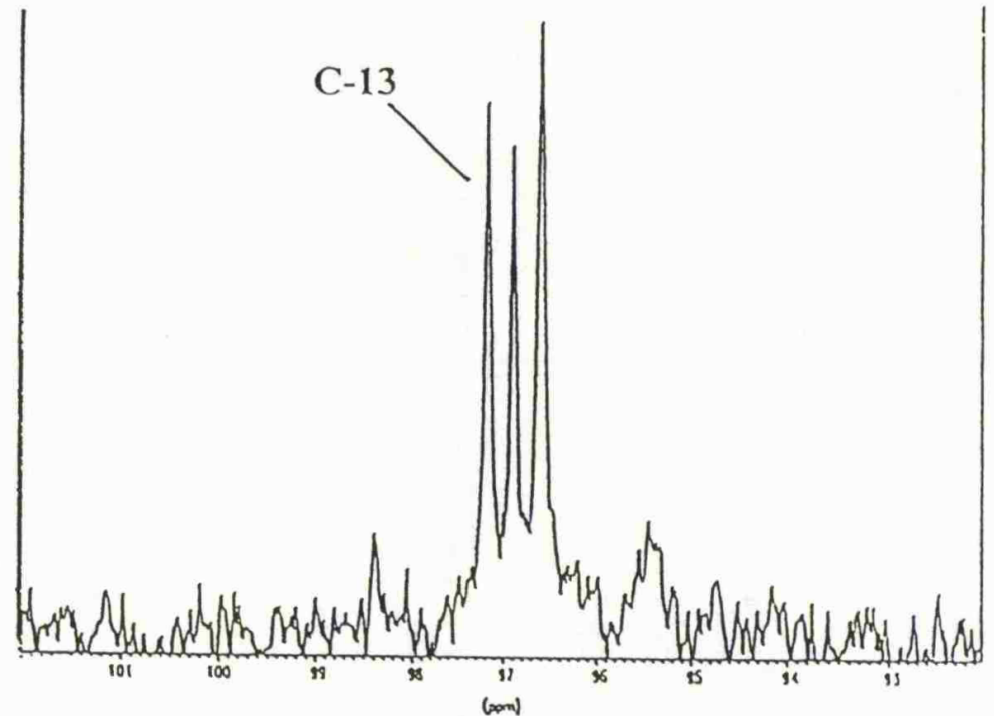

(c)

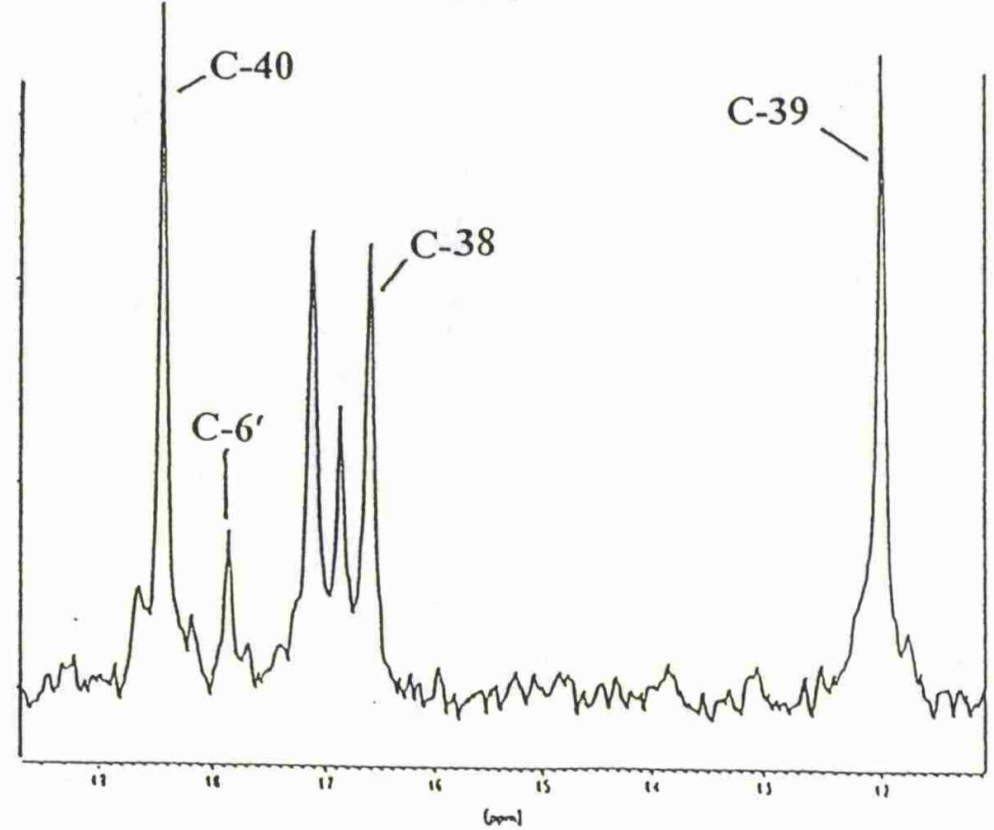

Fig. 4.6 Specified peaks from the ${ }^{13} \mathrm{C}\left\{{ }^{1} \mathrm{H}\right\}$ NMR spectrum of amphotericin B obtained after the feeding of $\left[1,2-13 \mathrm{C}_{2}\right]$ acetate 
may be partly metabolised via the citric acid cycle and be incorporated into the metabolite as the propionate subunit. ${ }^{159}$

To determine whether the propionate peaks were indeed enhanced, a comparison was made with the signals corresponding to the carbons of the sugar moiety. The sugar group is not expected to be derived from acetate and so these resonances that are distinguishable (at 17.8, 56.0 and $68.7 \mathrm{ppm}$ ) can act as a measure of enhancement. Comparing the ratio of the peaks corresponding to both $\mathrm{C}-39$ and $\mathrm{C}-40$ with that of C- $6^{\prime}$ in the labelled and unlabelled spectra, indicates that these peaks have been enhanced greatly. A comparison of the peak corresponding to C-16 (58.4 ppm) and the adjacent sugar carbon resonance $(56.0 \mathrm{ppm})$, also indicates a possible enhancement, as the sugar peak has almost been lost in the noise level of this spectrum. It is apparent that the labels have been incorporated into positions of the propionate subunits to differing extents, with the resonances corresponding to the methyl groups (C-3 of propionate) being enhanced by a factor of at least four, compared to the unlabelled sample.

In an attempt to calculate the overall percentage incorporation of $\left[1,2-{ }^{13} \mathrm{C}_{2}\right]$ acetate, electrospray mass spectrometry was employed, although this technique does not provide any information about the positions of incorporation. In Fig 4.7 the unlabelled and labelled mass spectra can be compared. The overall percentage incorporation of the labelled acetate into amphotericin B can be determined quantitatively by calculating the percentage increase in peak height in relation to the 
natural abundance (Table 4.3). As would be expected the $M+2$ peak has increased the most due to incorporation of the two ${ }^{13} \mathrm{C}$ labels from a single intact di-labelled acetate unit. However, there is also evidence for the presence of species from incorporation of two, of three and of four di-labelled acetate units $(M+4,6$ and 8 , respectively), along with further evidence for the incorporation of singly labelled subunits $(M+1,3,5,7$ and 9) arising from fragmented acetate sub-units, either into acetate or propionate positions.

Evidently, there has been incorporation of the di-labelled acetate into the amphotericin B that was produced, but what proportion of these molecules of amphotericin B actually contain labels? For those molecules of amphotericin with no incorporated labels, we would expect a certain intensity of $M+1, M+2$ etc. peaks in the mass spectrum due to the natural abundance of $1 \%{ }^{13} \mathrm{C}_{1}$, the level of which would be fixed with time. From the feeding of the di-labelled acetate, there would be 'extra' ${ }^{13} \mathrm{C}_{1}$ and ${ }^{13} \mathrm{C}_{2}$ in the acetate pool, the levels of which would vary with time due to the pulsed-feeding, with incorporation into the metabolite for a ${ }^{13} \mathrm{C}_{1}$ unit being $c a$. $2 \%$ and for a ${ }^{13} \mathrm{C}_{2}$ unit being ca. $10 \%$ (Table 4.3). If the labels are randomly incorporated into all the molecules of amphotericin $\mathrm{B}$, the experimental values for the percentage incorporation will be similar to the statistical values. However, it is possible that the pulsed-feeding technique used for administering the labels may have resulted in some molecules of amphotericin B becoming highly labelled, with others incorporating less than statistical values. This would occur due to the very high 
(a)

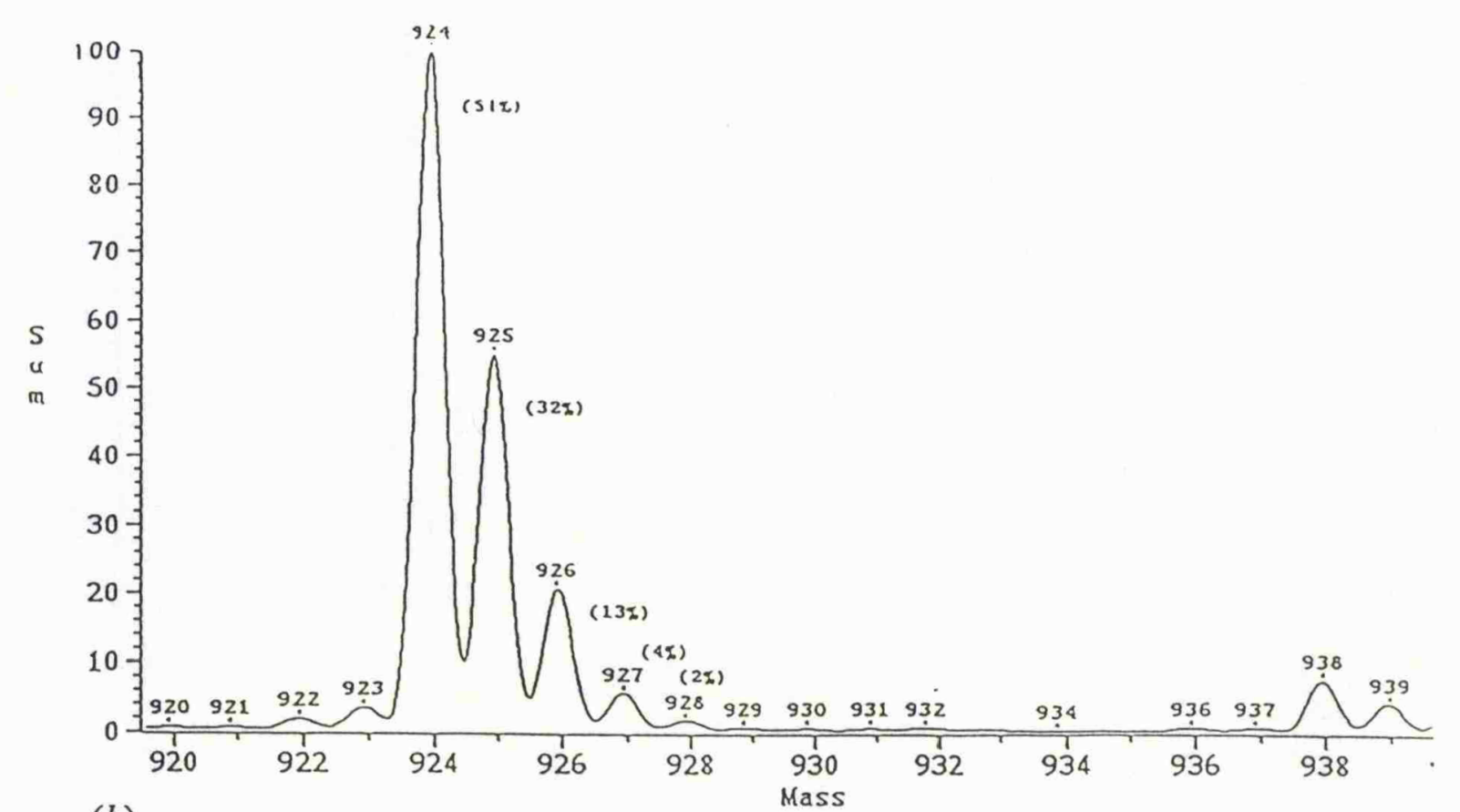

(b)

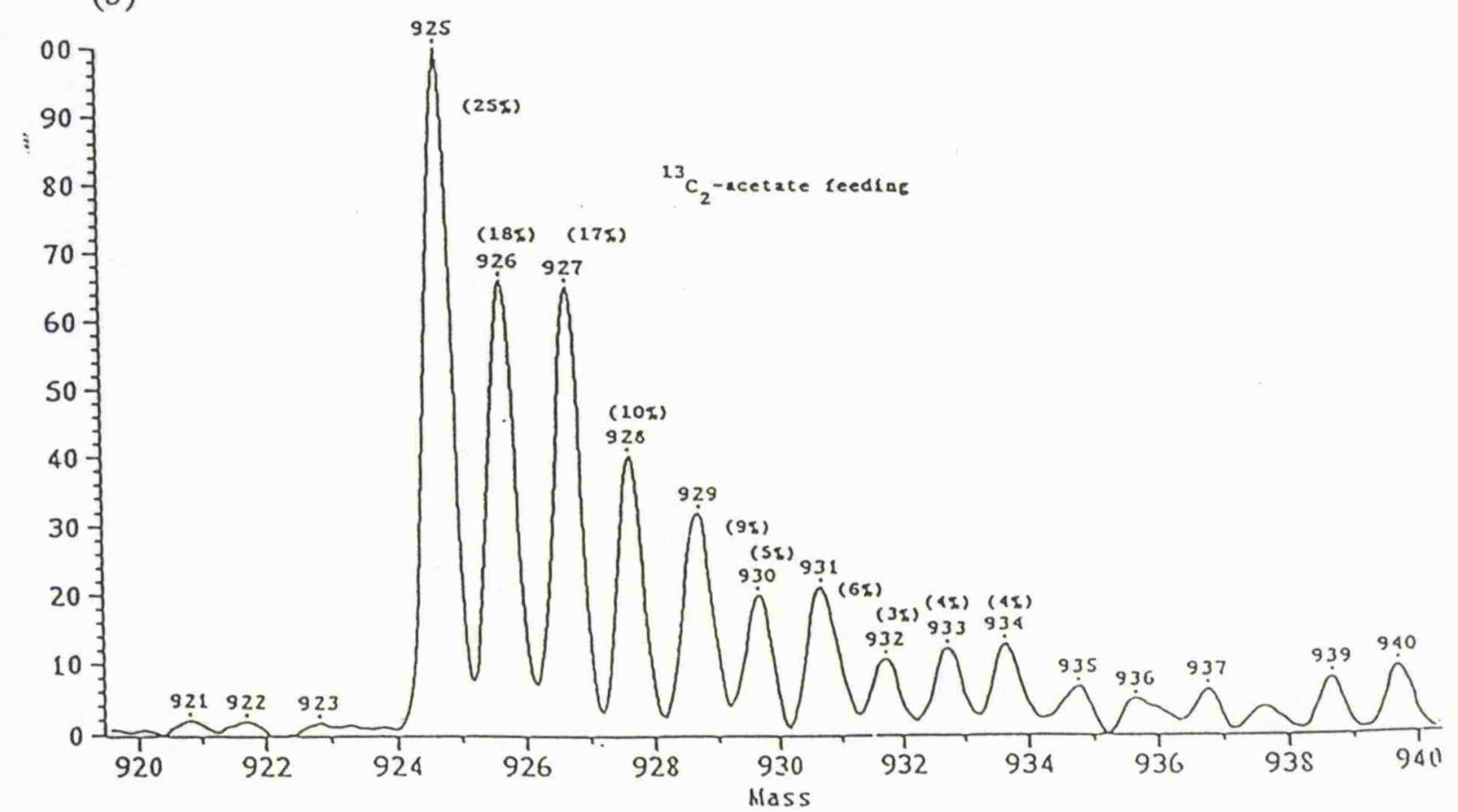

Fig. 4.7 Comparison of the electrospray mass spectrum of amphotericin $B$;

(a) unlabelled and $(b)$ after the feeding of $\left[1,2-{ }^{13} C_{2}\right]$ acetate; for $(a)$ and $(b) 924 \equiv 925$ 
Table 4.3 Calculation of the expected percentage increase in peak height for the labelled electrospray mass spectrum of amphotericin B

\begin{tabular}{|c|c|c|c|c|c|}
\hline Peak & & $\begin{array}{l}\text { Unlabelled } \\
\text { sample(\%) }\end{array}$ & $\begin{array}{l}\text { Natural } \\
\text { abundance }^{b}\end{array}$ & $\begin{array}{l}\text { Labelled } \\
\text { sample }(\%)^{a}\end{array}$ & $\begin{array}{r}\text { Increase } \\
(\%)\end{array}$ \\
\hline 924 & $\mathrm{M}$ & 51 & 25 & 25 & - \\
\hline 925 & $M+1$ & 32 & 16 & 18 & 2 \\
\hline 926 & $M+2$ & 13 & 6 & 17 & 11 \\
\hline 927 & $M+3$ & 4 & 2 & 10 & 8 \\
\hline 928 & $M+4$ & $<2$ & $<1$ & 9 & 9 \\
\hline 929 & $M+5$ & $<1$ & $<1$ & 5 & 5 \\
\hline 930 & $M+6$ & $<1$ & $<1$ & 6 & 6 \\
\hline 931 & $M+7$ & $<1$ & $<1$ & $\sim 3$ & $\sim 3$ \\
\hline 932 & $M+8$ & $<1$ & $<1$ & $\sim 4$ & $\sim 4$ \\
\hline 933 & $M+9$ & $<1$ & $<1$ & $\sim 3$ & $\sim 3$ \\
\hline
\end{tabular}

$a$ Values are $\%$ of total peaks. ${ }^{b}$ Calculated by allowing $M$ (natural abundance) $=25 \%$.

levels of labelled acetate being present in a cell just after the pulsed-feeding, thus producing amphotericin B that is highly labelled, with the subsequent amphotericin $\mathrm{B}$ produced containing little or no label. The next feeding would once again result in a number of molecules of amphotericin B being highly labelled etc. The effect of this pulsed-feeding would result in values for the percentage incorporation that would be very different from the statistical values for random incorporation, as noted by Vederas et al. with the incorporation of labelled acetates, ${ }^{160}$ who were able to detect inter-unit coupling between adjacent labelled acetate units. 
Thus, the statistical values for the probability of the produced amphotericin B molecules containing one di-labelled acetate unit, two di-labelled units, three dilabelled units etc. can be seen in Table 4.4, with these calculations based on the probability of any acetate being 10\% di-labelled (17 positions, each $10 \%)$. For this calculation, the $\mathrm{M}+3$ value actually corresponds to the incorporation of one dilabelled unit plus a singly labelled unit and thus was included in the category denoting one intact di-labelled acetate unit. Likewise, the values for $M+5,7$ and 9 were included in the categories for 2, 3 and 4 intact di-labelled acetates, respectively.

In this calculation, it is assumed that all molecules without labels $(\mathrm{M}+0)$ were assembled when the concentration of labelled acetate was very low, i.e. before addition or between pulsed-feedings. This represents $M(25), M+1(16), M+2$ (6) etc. using the $1 \%$ natural abundance of ${ }^{13} \mathrm{C}$ (Table 4.3). This represents $c a .50$ molecules out of every $c a .100$ and of the remaining ca. 50 molecules, 27 have one intact ${ }^{13} \mathrm{C}_{2}$ acetate $(M+2$ and $M+3), 14$ have two intact ${ }^{13} C_{2}$ acetates $(M+4$ and $M+5), 9$ have three intact ${ }^{13} \mathrm{C}_{2}$ acetates $(M+6$ and $M+7)$ and $\sim 7$ have four intact ${ }^{13} \mathrm{C}_{2}$ acetates $(M+8$ and $\mathrm{M}+9)$.

Now if, of this $50 \%$ of labelled molecules there is a steady state chance of 1 in 10 acetates being di-labelled, the statistical probability of no intact di-labelled acetates, one intact di-labelled acetate, two, three, etc. being incorporated is calculated as in Table 4.4. 
Table 4.4 Calculation for the incorporation of di-labelled acetates into the $50 \%$ of labelled molecules of amphotericin B

\begin{tabular}{llll}
\hline $\begin{array}{l}\text { Intact di- } \\
\text { labelled } \\
\text { acetate } \\
\text { units }\end{array}$ & Calculation & $\begin{array}{l}\text { Statistical } \\
\text { probability } \\
1100\end{array}$ & $\begin{array}{l}\text { Value for the } \\
50 \% \text { labelled } \\
\text { molecules } / 50\end{array}$ \\
\hline 0 & $(0.9)^{17}$ & 17 & 8.5 \\
1 & $17 \times 0.1 \times(0.9)^{16}$ & 31.5 & 16 \\
2 & {$[(17 \times 16) / 2] \times(0.1)^{2} \times(0.9)^{15}$} & 28 & 14 \\
3 & {$[(17 \times 16 \times 15) /(3 \times 2 \times 1)] \times(0.1)^{3} \times(0.9)^{14}$} & 15.5 & 7.5 \\
4 & {$[(17 \times 16 \times 15 \times 14) /(4 \times 3 \times 2 \times 1)] \times(0.1)^{4} \times(0.9)^{13}$} & 6 & 3 \\
\hline
\end{tabular}

The figures in the last column thus give the values for $10 \%$ statistical labelling for those molecules containing labels, and these values added to those of the $50 \%$ of molecules with natural abundance levels of ${ }^{13} \mathrm{C}$, give the overall figures for the statistical value (Table 4.5).

The figures for the expected and actual values for the probability of intact incorporation of di-labelled acetate units in Table 4.5 do not appear to be statistically different, and thus there is a random incorporation of label into all the molecules of amphotericin B. However, as the relative peak height of $M+8$ and $M+9$ does 'appear' high, a small percentage of molecules might be over labelled statistically. As with all these calculations, the values indicated are all estimates. 
Table 4.5 Comparison of the statistical and experimental values for the intact incorporation of di-labelled acetate units

\begin{tabular}{|c|c|c|}
\hline $\begin{array}{l}\text { Intact di-labelled } \\
\text { acetate units }\end{array}$ & $\begin{array}{l}\text { Statistical } \\
\text { probability (\%) }\end{array}$ & $\begin{array}{l}\text { Experimental } \\
\text { value }(\%)\end{array}$ \\
\hline 0 & 0.50 & 0.43 \\
\hline 1 & 0.24 & 0.27 \\
\hline 2 & 0.15 & 0.14 \\
\hline 3 & 0.085 & 0.09 \\
\hline 4 & 0.004 & 0.07 \\
\hline
\end{tabular}

\subsection{2 $\left[1-{ }^{13} \mathrm{C}\right]$ Acetate and $\left[2-{ }^{13} \mathbb{C}\right]$ acetate}

A similar experiment was undertaken in the feeding of $\left[1-{ }^{13} \mathrm{C}\right]$ and $\left[2-{ }^{13} \mathrm{C}\right]$ acetate to $500 \mathrm{~cm}^{3}$ shake flasks with the aim of confirming the assignment of some of the resonances by ${ }^{13} \mathrm{C}$ NMR in the double bond region and also, due to the high incorporation levels in the previous experiment, with the possible observation of inter-unit coupling. The protocol used was the same as previously, with the exception that $1 \mathrm{~g}$ of each acetate was added to three flasks over $70 \mathrm{~h}(42 \mathrm{mg}$ of each acetate in $200 \mu \mathrm{l}$ of sterilised water was added to each flask at $18,22,26,42,46,50$, 66 and $70 \mathrm{~h}$ after inoculation). A fourth flask was inoculated and grown as a control, 
without the addition of any compounds. The result of the UV assay of the whole broth over the production time-course to determine the metabolite titres can be seen in Fig. 4.8. The concentration of amphotericin B was monitored in order to determine whether normal production occurred during the experiment. The UV assay at $48 \mathrm{~h}$ appeared normal by comparison to the control and so the addition of acetate was continued (if the microorganism was not producing amphotericin as normal at this stage, the addition of the costly labelled compounds would have been halted). After $72 \mathrm{~h}$, the whole broths from the three flasks were pooled and extracted in the normal manner, but it was not until this time that the final assay was taken and as can be seen from Fig. 4.8, the concentration of amphotericin B was discovered not to be as high as expected.

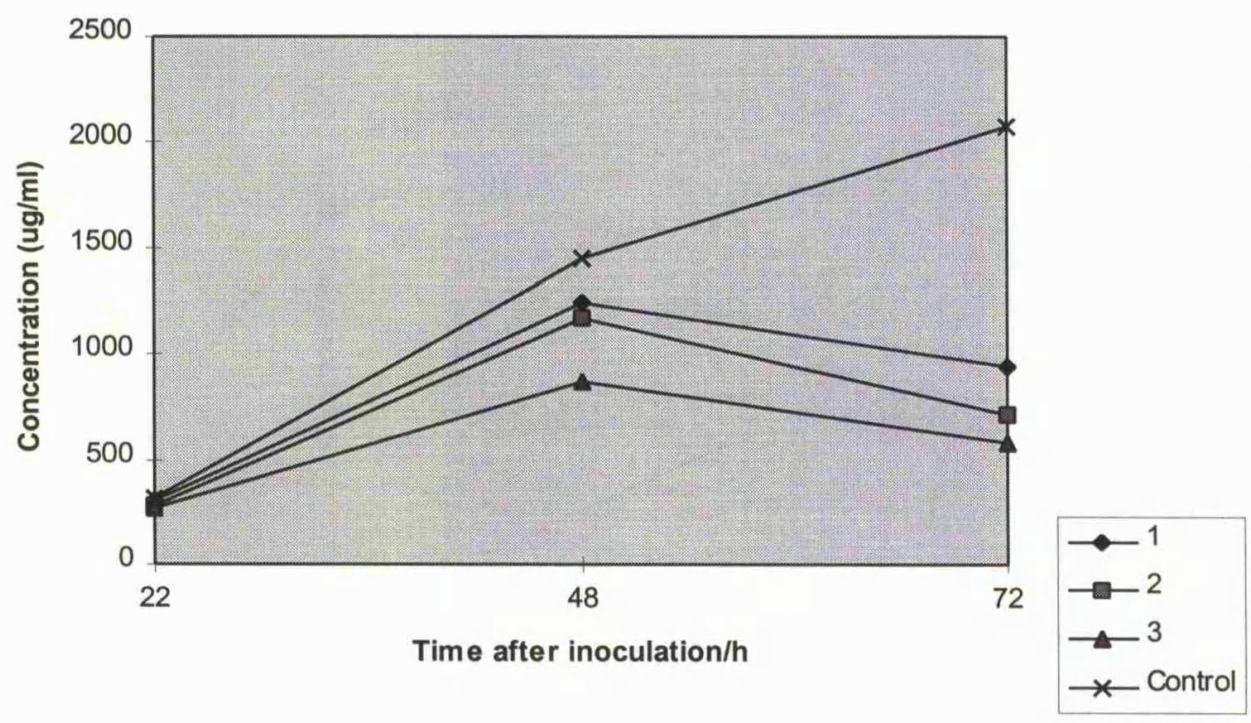

Fig. 4.8 UV assay of the fermentation broth for the addition of $[1-13 \mathrm{C}]$ and $\left[2-{ }^{13} \mathrm{C}\right]$ acetate $(1,2$ and 3$)$ compared to the control 
Unfortunately, the NMR spectrum obtained from the $100 \mathrm{mg}$ of amphotericin B precipitated did not show any significant incorporation of the labelled acetate, which may be due to the fact that the production was not as expected. This may possibly be due to some contamination in the flasks, although this seems unlikely as the control flask produced satisfactorily. Another explanation may concern the levels of acetate that were administered to the flasks ( $2 \mathrm{~g}$ between three flasks), which was a much greater concentration than in the experiment involving doubly labelled acetate (in which $1 \mathrm{~g}$ was divided between four flasks). The reason for this higher level of administered acetate was in order to attempt a greater incorporation, as high levels were required in order to observe inter-unit coupling.

\subsection{3 $\left[1{ }^{13} \mathrm{C}\right]$ Acetate and diethyll $\left[2{ }^{-13} \mathrm{C}\right]$ malonate}

This second experiment involving two singly labelled precursors was undertaken in the hope of gaining further evidence for the arrangement of the sub-units of the structural skeleton of amphotericin B, with the possibility of detecting some interunit coupling. The feeding experiment was similar to those described previously. However, due to the failure of the singly labelled acetate feedings above, $1 \mathrm{~g}$ in total of labelled compound was added to four flasks (similar conditions to the successful doubly labelled acetate experiment). Thus, $\left[1{ }^{13} \mathrm{C}\right]$ acetate and diethyl [2${ }^{13} \mathrm{C}$ ]malonate $(0.5 \mathrm{mg}$ of each) were added to four shake flasks over a period of $72 \mathrm{~h}$ (21 $\mathrm{mg}$ of each in $100 \mu \mathrm{l} \mathrm{H} \mathrm{H}_{2} \mathrm{O}$ per feeding per flask at 21, 25, 42, 46, 50 and $66 \mathrm{~h}$ after inoculation). The UV assay of amphotericin B production over this time-course 
can be seen in Fig. 4.9. The titres of antibiotic production in this experiment were lower than previously, however all flasks showed a similar production profile including the control flask, so the lower levels of metabolite production did not appear to be due to any adverse effects of the added labelled compounds. Towards the end of the time-course however, the levels once again increased, which suggests the microorganism was growing and producing satisfactorily. After this time, the contents of the four flasks were pooled and extracted in the usual manner with ethyl acetate and Aliquat. The precipitation procedure was followed by UV assay (Table 4.6) and yielded 150-200 mg of the fine yellow solid of amphotericin B.

The ${ }^{13} \mathrm{C}\left\{{ }^{1} \mathrm{H}\right\}$ NMR spectra from the extracted amphotericin B can be seen in Fig. 4.10. At first glance, it is evident that there has been an apparent enhancement of certain resonances, indicating the incorporation of labels at the corresponding

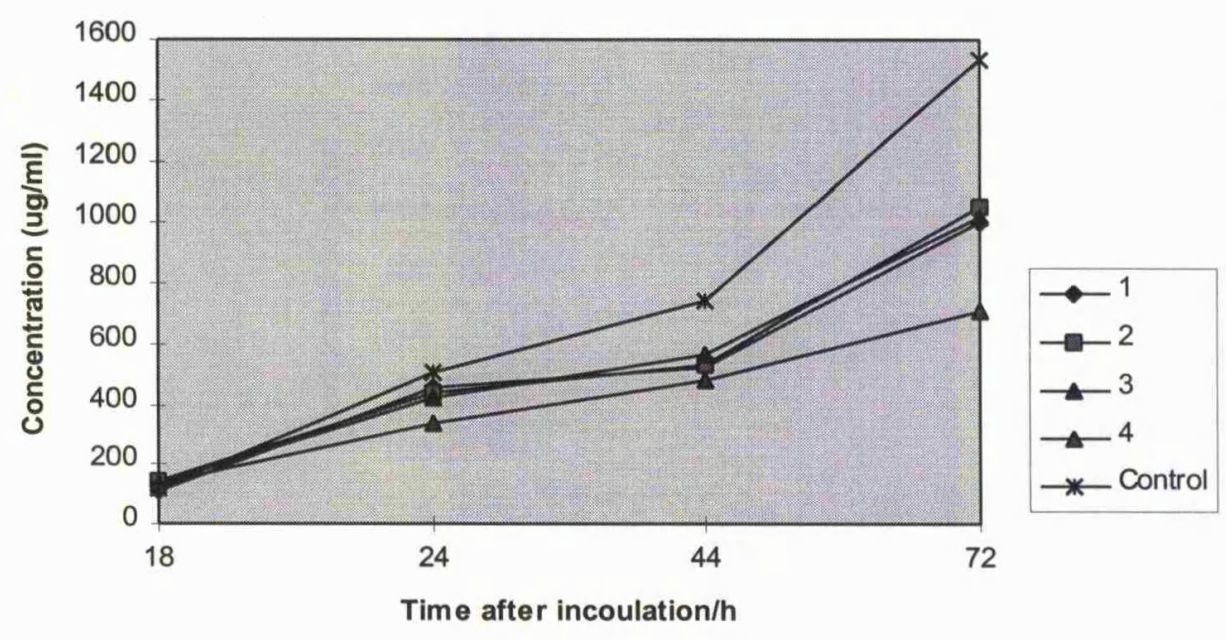

Fig. 4.9 UV assay of the fermentation broth after the addition of $\left[1-{ }^{13} \mathrm{C}\right]$ acetate and diethyl $[2-13 \mathrm{C}]$ malonate $(1,2,3,4)$ compared to the control 
Table 4.6 UV assay of the precipitation process of amphotericin B from the ethyl acetateAliquat extract of $4 \times 60 \mathrm{~cm}^{3}$ whole broth after the addition of $0.5 \mathrm{~g}[1-13 \mathrm{C}]$ acetate and 0.5 g diethyl $[2-13$ C]malonate

\begin{tabular}{|c|c|c|c|}
\hline Day & $\mathrm{pH}$ & Concentration/ $\mu \mathrm{g}$ & \\
\hline & & Amphotericin $\mathrm{A}^{a}$ & Amphotericin $\mathrm{B}^{b}$ \\
\hline 1 & 9.7 & 1235 & 1310 \\
\hline 4 & 7 & 1150 & 464 \\
\hline 5 & 7 & 1034 & 321 \\
\hline 6 & 7 & 1120 & 298 \\
\hline
\end{tabular}

positions in the metabolite. The intensity of resonances of those carbons deriving from the carbonyl carbon of acetate are all significantly enhanced, which are the signals that would be expected to be increased due to the incorporation of [1${ }^{13} \mathrm{C}$ ]acetate, and show an overall $4 \%$ enhancement. If the labelled diethyl malonate were to be incorporated, the signals corresponding to the carbons in the adjacent positions to these would be expected to be enhanced. However, these signals do not appear to have increased at all (e.g. the signal for C-38 at $16.8 \mathrm{ppm}$ or for $\mathrm{C}-2,4,12$ or 14 in the region of $42-46 \mathrm{ppm}$ ) when compared to the sugar carbon resonances. It is also apparent that no label was incorporated into any of the propionate units from the lack of enhancement of any of the corresponding peaks. It is unclear as to why the feeding of labelled diethyl malonate failed to produce any significant incorporation. This experiment was repeated under identical conditions and a similar level of incorporation of the labelled acetate was detected but once again, with no apparent incorporation of the diethyl malonate label. It may be a possibility that the 


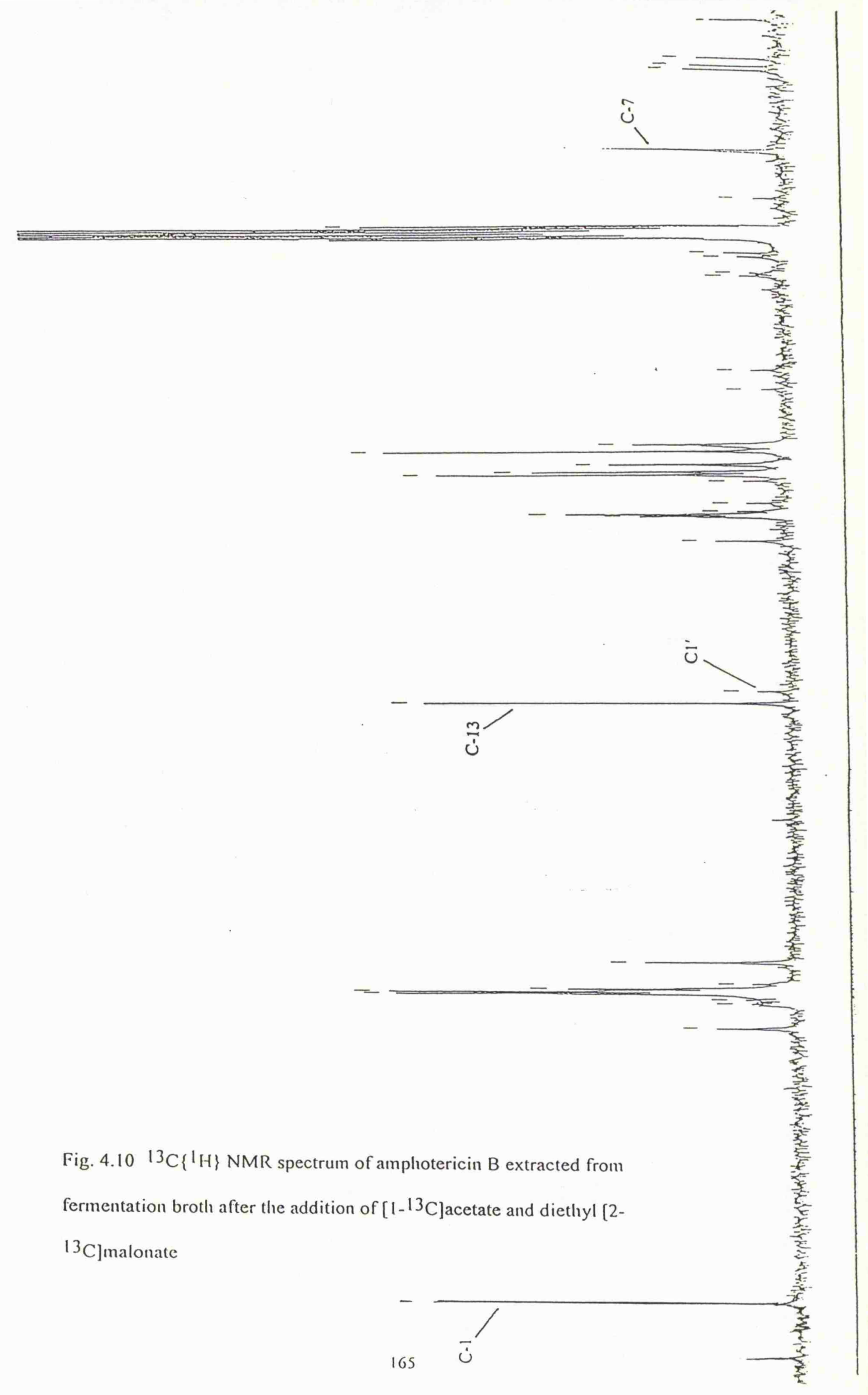


solubility of diethyl malonate in the medium varies greatly from that of acetate, thus preventing it from being incorporated as readily by the cell. Alternatively, the $\mathrm{pH}$ of the system may affect the uptake of diethyl malonate as it may need to be hydrolysed first for solubility.

Thus, these feeding experiments have provided firm evidence for the incorporation of labelled acetate into amphotericin B. The pattern of incorporation of the singly and doubly labelled acetate units into the metabolite are as would be expected for the acetate-derived positions of the structure of amphotericin B and their incorporation has provided some evidence for the anticipated biosynthetic scheme of assembly of amphotericin B. Further evidence was provided with investigations that were run in parallel with this study, in which the incorporation of $\left[1-{ }^{13} \mathrm{C}\right]$ and $\left[3-{ }^{13} \mathrm{C}\right]$ propionate into amphotericin B was detected by ${ }^{13} \mathrm{C}\left\{{ }^{1} \mathrm{H}\right\}$ NMR. ${ }^{161}$ These studies have proved invaluable in developing a protocol for the administration of labelled compounds, which may be employed in any further biosynthetic studies involving amphotericin B.

\subsection{Diketide feeding}

An alternative fermentation procedure was investigated for the diketide feeding experiment, as discussed in the previous chapter. This experiment was of a different nature than the pulsed-feeding experiments described above. The diketide feeding was carried out on a much smaller scale, in $100 \mathrm{~cm}^{3}$ shake flasks containing only 15 
$\mathrm{cm}^{3}$ of production medium. The reason for using such reduced volumes was that there was only a small amount of the labelled diketide available and therefore it was to be fed in one portion; the use of smaller volumes of whole broth, along with the calculation of the optimum feeding time, would increase the chances of the labelled precursor being incorporated into the metabolite. However, previous such studies have encountered problems in which the precursor either did not get into the cell and/or was degraded by the fatty acid oxidases present in the cell etc. ${ }^{162}$ In an attempt to overcome such problems, various compounds, in particular $\beta$-oxidation inhibitors which would hopefully suppress any precursor degradation, have been added to fermentation broths. ${ }^{33}$ Other problems such as the solubility and uptake of the precursor must be optimised and the use of 2,6-O-dimethyl- $\beta$-cyclodextrin (DMCD 33) in studies of the biosynthesis of monensin A, was shown to stimulate the production of this metabolite and possibly also the uptake of the precursor into the cells. ${ }^{36}$ Thus, in this study, three compounds were added to the fermentation flask in addition to the labelled diketide; two $\beta$-oxidation inhibitors, 4-pentynoic acid 31 and 3-(tetradecylthio)propanoic acid 32, ${ }^{32-35}$ along with DMCD 33. 


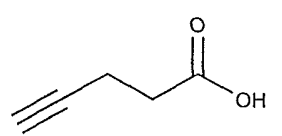

31

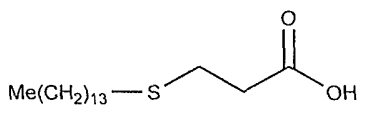

32

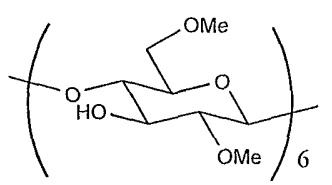

33

A preliminary experiment was undertaken in order to determine whether the addition of such compounds had any adverse effects on the production of amphotericin B in the fermentation media. Inoculation of $100 \mathrm{~cm}^{3}$ shake flasks containing $15 \mathrm{~cm}^{3}$ of FCA media was carried out as described in the previous chapter, in which the optimum time for feeding any precursors was determined to be $c a .28 \mathrm{~h}$ after inoculation. Thus, at the appropriate time in the preliminary experiment, the compounds were added to the production medium aseptically to each of four shake flasks (15 mg 31 and 33 , each in $200 \mu$ of sterilised $\mathrm{H}_{2} \mathrm{O}$, and $15 \mathrm{mg} 32$ as a solid per flask). A UV assay time-course of the amphotericin B production was measured, the results of which can be seen in Fig. 4.11 (four flasks were inoculated, with Fig. 4.11 representing the mean value). The results show good comparison to previous whole broth time-course production profiles, and so it appears that the addition of these compounds to the medium does not adversely affect the production of the metabolite. 


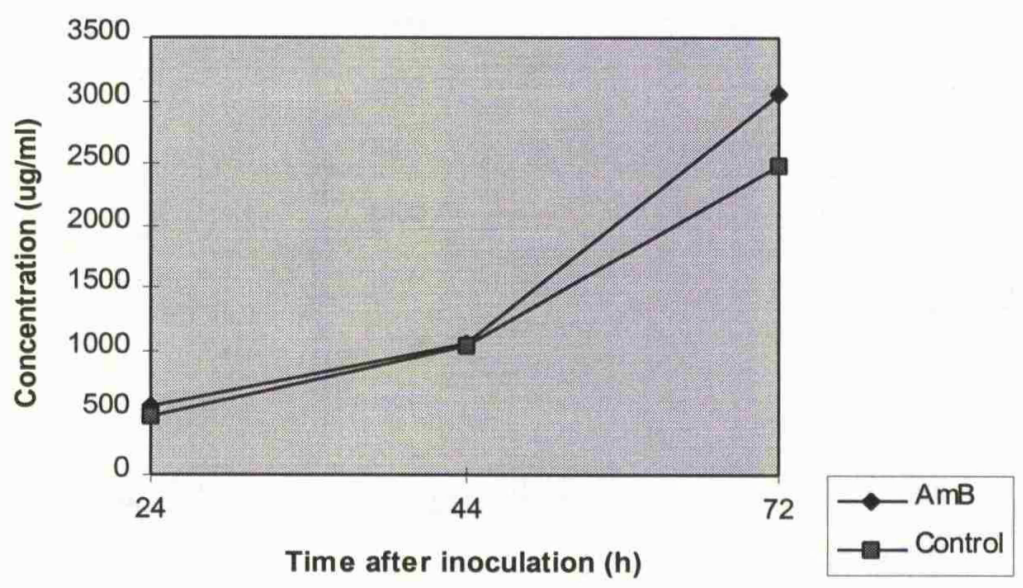

Fig. 4.11 UV assay of amphotericin B production after the addition of DMCD, 4-PA and 3-TDPA

The groundwork had thus been carried out for the feeding of the diketide labelled precursor. In the previous chapter, the whole broth time-course for $100 \mathrm{~cm}^{3}$ fermentation flasks containing $15 \mathrm{~cm}^{3}$ of broth was measured, the result of which was the determination of the conditions for the growth of the microorganism and the optimum time for feeding the precursor ( $c a .28 \mathrm{~h}$ after inoculation). The extraction procedure was also determined, with satisfactory electrospray mass spectra being obtained from isolated samples of amphotericin A and B.

For the diketide labelling experiment, four $100 \mathrm{~cm}^{3}$ shake flasks containing $15 \mathrm{~cm}^{3}$ FCA media were inoculated as usual and the whole broth was assayed (Fig. 4.12) in order to ensure that the production of amphotericin B had commenced as expected before the addition of any compounds took place. To the first flask, the labelled 
diketide (51, $10 \mathrm{mg}, 200 \mu \mathrm{l}$ ethanol) was added (28 h after inoculation), plus the additional compounds $(31,32,33)$. Under exactly the same conditions, the control epimeric labelled diketide (133), as well as the additional compounds, were fed to the second flask. As control experiments, a flask of whole broth was grown that had only the three extra compounds added, along with a flask that did not have any added compounds. The levels of amphotericin B production were monitored after the addition of the compounds, with the production profile over the time-course of the experiment being as expected (Fig. 4.12). The UV assay also showed the expected levels of amphotericin A production.

The whole broth from shake flasks 1 (labelled diketide 51) and 2 (control labelled epimeric diketide 133) were then extracted as described in the previous chapter. The respective freeze-dried whole broth methanol extractions were evaporated in vacuo and the yellow residue was redissolved by sonication in a solution of methanol ( 4 $\mathrm{cm}^{3}$ ) and DMSO $\left(1 \mathrm{~cm}^{3}\right)$. Once again some of the yellow residue did not dissolve and so the mixture was centrifuged, the supernatant removed and the remaining solid redissolved in methanol $\left(2 \mathrm{~cm}^{3}\right)$ and DMSO $\left(0.5 \mathrm{~cm}^{3}\right)$. The two extracts for each of the shake flasks were then assayed by UV to determine the content of amphotericin 


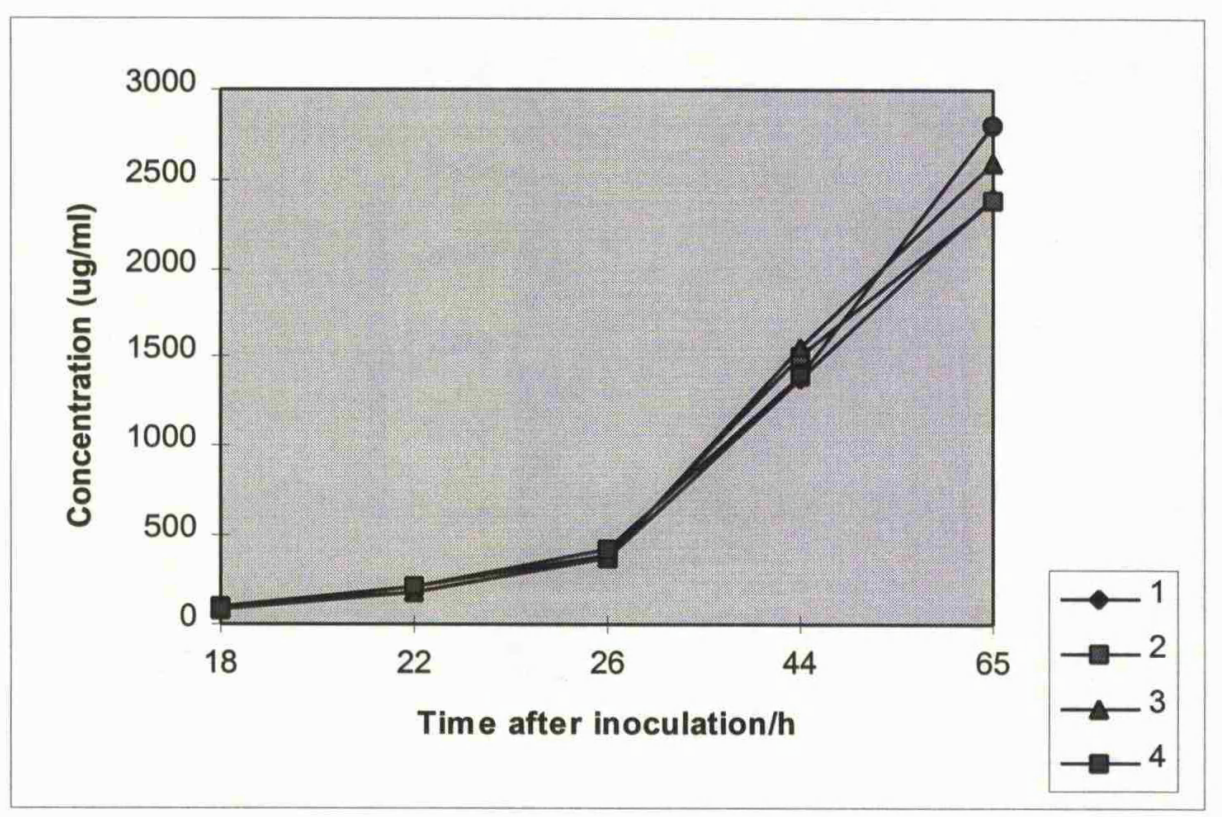

Fig. 4.12 UV assay of amphotericin B production in the various shake flasks in the diketide feeding experiment. Compounds added: 1, labelled diketide 51, 31, 32 and 33;2, epimeric control diketide 133 plus $\mathbf{3 1}, \mathbf{3 2}$ and $33 ; 3, \mathbf{3 1}, \mathbf{3 2}$ and $33 ; 4$, none (control).

A and B. These results can be seen in Table 4.7, with the initial extract containing similar quantities of amphotericin $\mathrm{A}$ and $\mathrm{B}$ and the second extract containing a majority of amphotericin B.

The samples were then injected onto the HPLC, under the exact conditions as described previously and the peaks corresponding to amphotericin A and B were detected at $\lambda 305$ and $405 \mathrm{~nm}$, respectively. The fractions containing amphotericin A and B were collected, de-salted and the solvent removed, redissolving each sample in methanol $\left(2 \mathrm{~cm}^{3}\right)$ in preparation for analysis by electrospray mass spectrometry. In 
Table 4.7 Determination of amphotericin concentration by UV assay for the whole broth freeze-dried methanol extracts for shake flask 1 (labelled diketide) and 2 (control diketide) Sample Concentration $/ \mu \mathrm{g} \mathrm{m} \mathrm{m}^{-1}$

Amphotericin A Amphotericin B

Labelled diketide:

$\begin{array}{llr}\text { Extract 1 } & 5864 & 5188 \\ \text { Extract 2 } & 547 & 1629\end{array}$

Control diketide:

Extract $1 \quad 6253 \quad 5969$

$\begin{array}{ll}\text { Extract } 2 \quad 630 & 1034\end{array}$

order to determine the concentration of each sample, once again a UV assay was taken (Table 4.8).

In Fig. 4.13, the electrospray mass spectra of unlabelled amphotericin A and B can be seen, which were run overnight at high resolution. If any of the administered labelled compounds become incorporated intact into amphotericin, an enhanced peak at the $M+4$ position would be expected. This unfortunately was not the case in either the desired or control diketide. The mass spectra of the extracted amphotericin A can 
Table 4.8 Determination of amphotericin concentrations of samples in methanol $\left(2 \mathrm{~cm}^{3}\right)$ for electrospray mass spectrometry after HPLC separation

\begin{tabular}{ll}
\hline Sample & $\begin{array}{l}\text { Concentration } / \mu \mathrm{g} \\
\text { per sample }\end{array}$ \\
\hline
\end{tabular}

Labelled diketide

Extract 1: $\quad$ AmB 656

$\operatorname{AmA} \quad 987$

Extract 2: $\mathrm{AmB} \quad 550$

$\mathrm{AmA}$

Control diketide

Extract 1: $\mathrm{AmB} \quad 316$

$\mathrm{Am} A \quad 630$

Extract 2: $\mathrm{AmB} \quad 159$

$\mathrm{AmA}$

be seen in Fig. 4.14, which show no significant increase at all. The mass spectra of the amphotericin B peaks are not as easy to interpret (Fig. 4.15), as the samples were contaminated with some amphotericin A. However, the relative levels of amphotericin B can be determined (by extrapolating with the levels of amphotericin A), with the $M+4$ peak showing no apparent increase in height.

Unfortunately these results provide no further evidence to corroborate our proposed biosynthetic pathway of amphotericin B. However, neither do they provide any evidence against this mechanism, which would have been the case if the control 
Sample Normal AMPH B

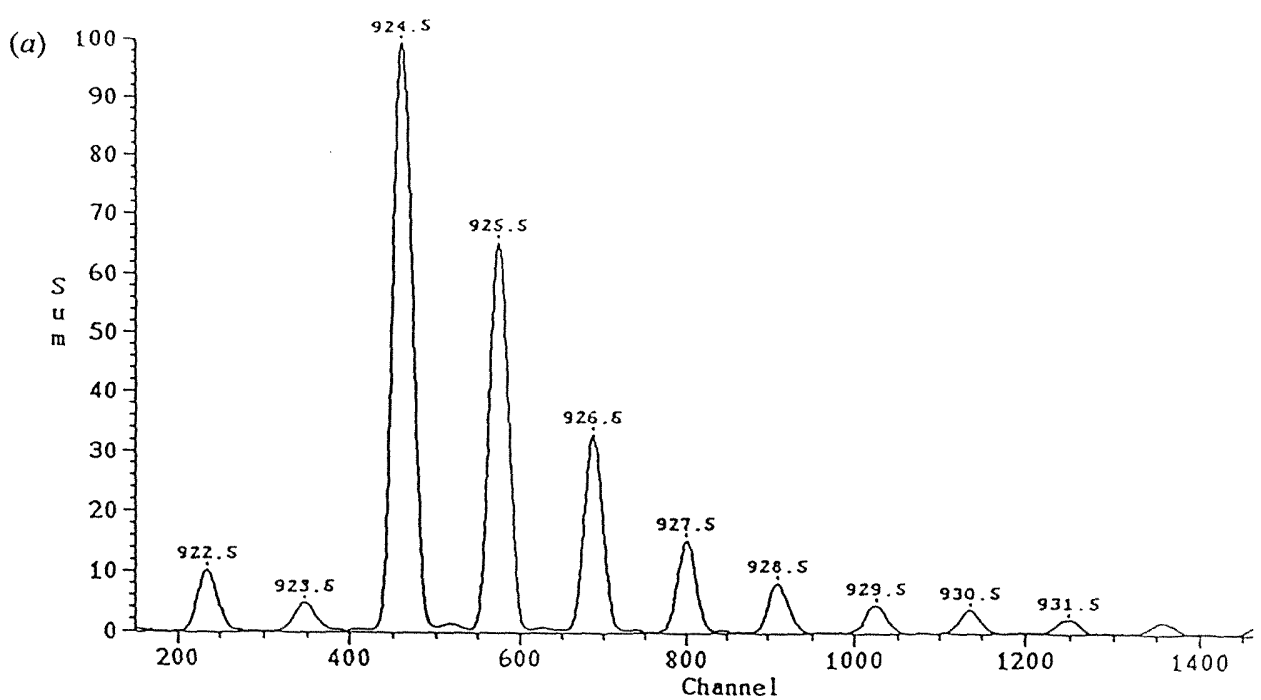

Sample AMPH A

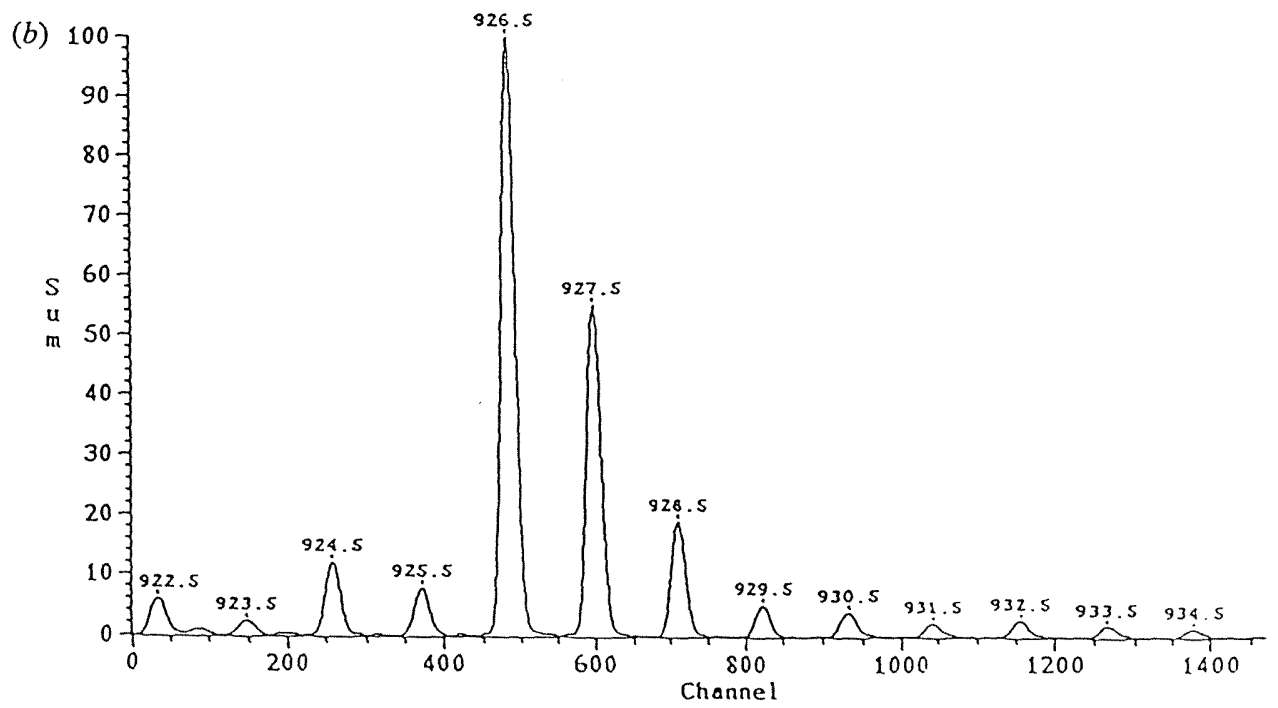

Fig. 4.13 Electrospray mass spectrum of a standard sample (a) amphotericin B and

(b) amphotericin $\mathrm{A}$ 

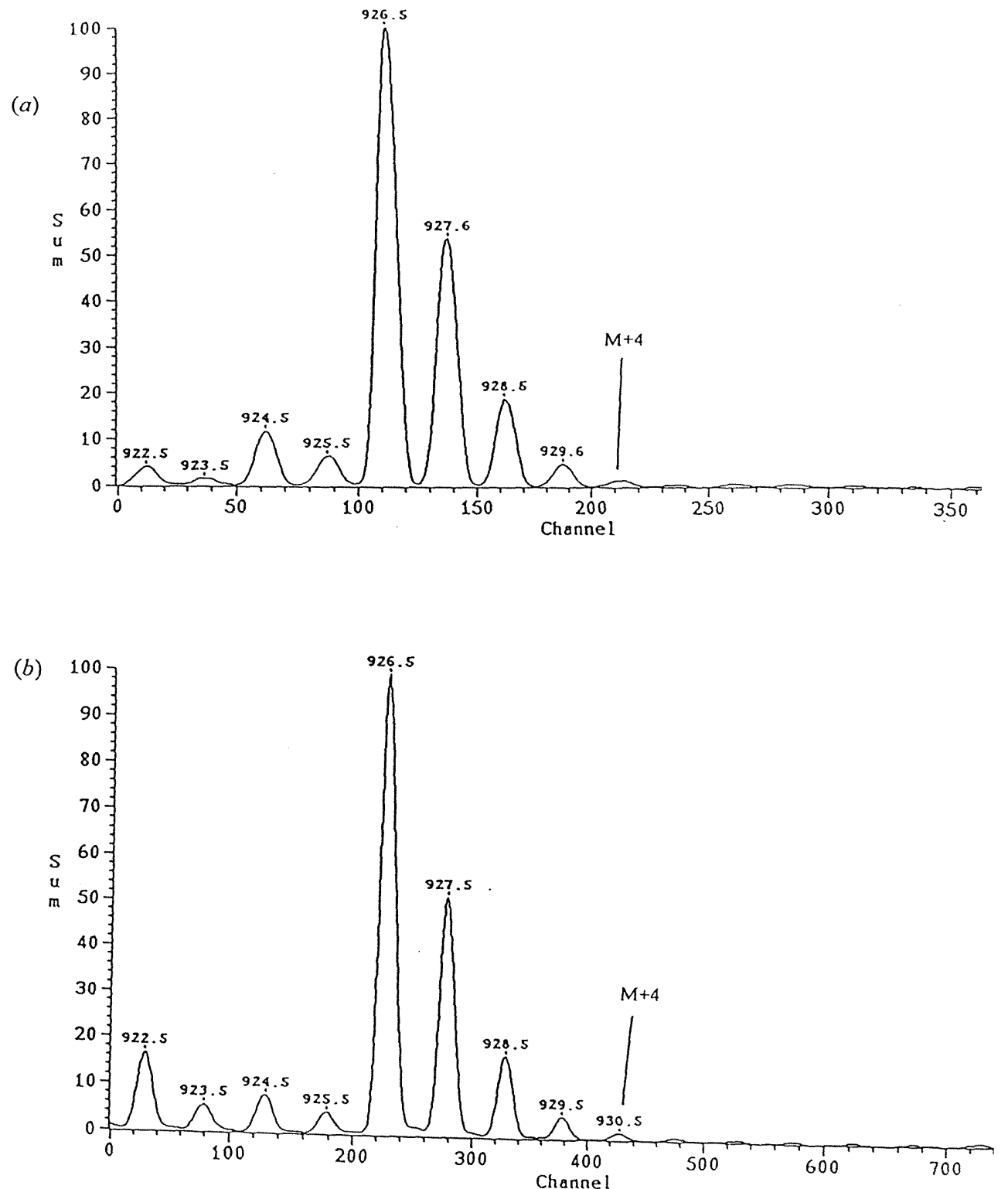

Fig. 4.14 Electrospray mass spectrum of the amphotericin A HPLC fraction from

(a) the diketide and $(b)$ control diketide feeding 
(a)

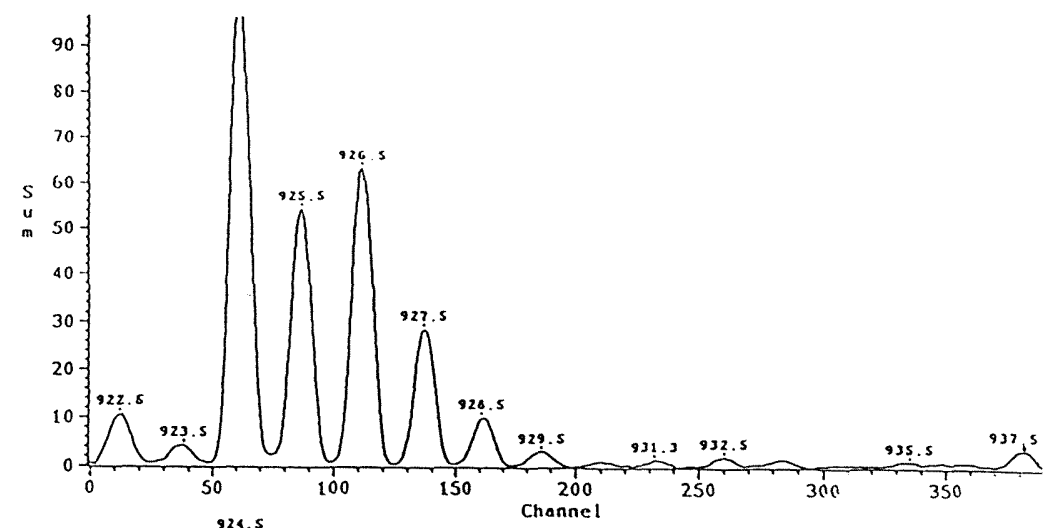

(b)

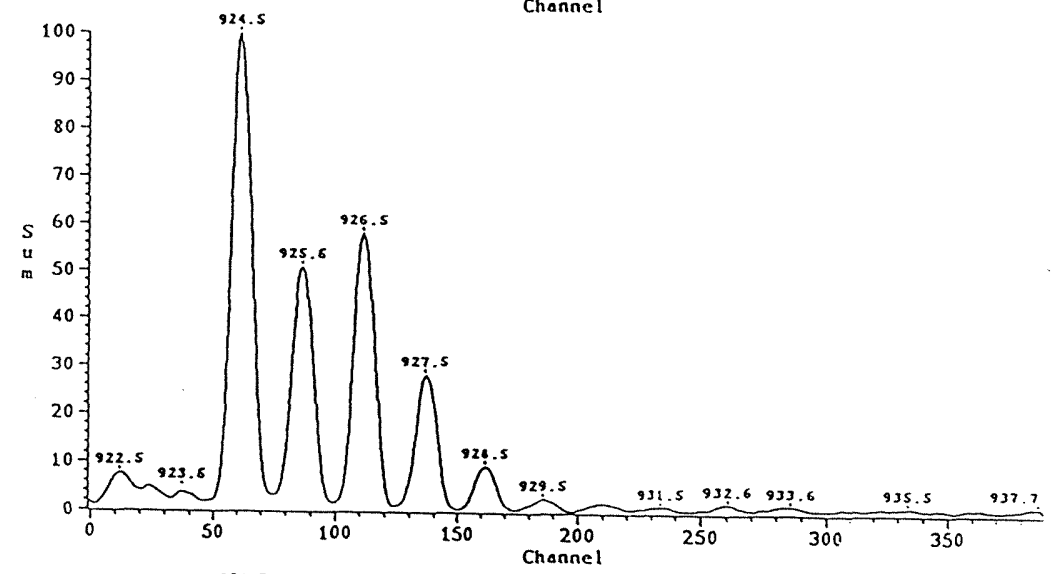

(c)

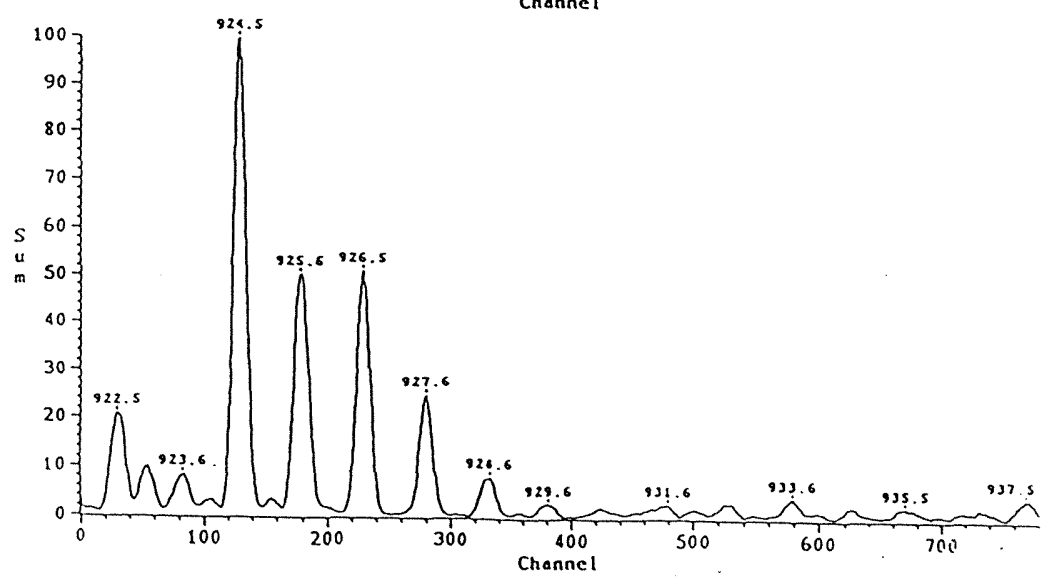

Fig. 4.15 Electrospray mass spectrum of the amphotericin B HPLC fraction from

(a) the labelled diketide, extract $1 ;(b)$ the labelled diketide, extract 2 ;

and $(c)$ the control diketide feeding 
labelled diketide had been incorporated. The detection of enhancement at the $\mathrm{M}+3$ peak in the ESMS would not negate the existence of the processive or non-processive assembly pathways but it would confirm the presence of active fatty acid oxidases. The procedure of the experiment itself appeared to be satisfactory with the whole broth titres comparing favourably to previous experiments under similar conditions. The extraction and purification procedures were also deemed successful as satisfactory electrospray mass spectra were obtained from the subsequent isolated samples. Other factors, including time of feeding, quantity of administered compound and successful uptake of the precursor through the bacterial cell wall, may all have had varying influential roles in determining the outcome of the experiment. Clearly, further investigations need to be undertaken.

The aim of this work was to increase our understanding into the biosynthesis of the important antifungal antibiotic amphotericin B. Unfortunately, the ultimate experiment in this study was rather inconclusive, however this work has certainly provided some evidence for the biosynthesis of amphotericin B with the successful incorporation of certain labelled compounds. This study has also laid the foundations for any future research involving amphotericin $B$, with the formulation of a satisfactory fermentation procedure, along with the determination of two very different extraction procedures. 


\section{Chapter 5}

\section{Summmary and fundure studies}

\subsection{Summary}

The aim of this project was to investigate the biosynthesis of the polyketide antibiotic amphotericin B and ultimately to provide evidence for the processive mode of assembly. This was carried out with a combination of chemical and biological experiments involving the feeding of variously labelled compounds to cultures of Streptomyces nodosus.

A possible biosynthetic pathway for amphotericin $\mathrm{B}$ was proposed, from which a putative precursor in the pathway at the diketide stage was targeted for synthesis. The presence of strategically placed stable isotopic labels in this compound was crucial for detection of any incorporation into amphotericin B. Evidence for the proposed biosynthetic pathway would be obtained by the incorporation of this and other labelled compounds, including $\left[{ }^{13} \mathrm{C}_{1}\right]$ - and $\left[{ }^{13} \mathrm{C}_{1}\right]$-acetates and diethyl $\left[{ }^{13} \mathrm{C}_{1}\right]$ malonate. 
Prior to the administration of labelled compounds to cultures of $S$. nodosus, an investigation into the growth of the microorganism and production of amphotericin B was undertaken in order to determine the optimum time for the feeding of precursors. For the feeding experiments, two very different extraction procedures were formulated.

A large scale extraction involving the use of a hydrophobic extractant ion was carried out which was highly selective and resulted in amphotericin B of high purity. This extraction process was used in the labelled acetate and diethyl malonate feeding experiments, with detection of incorporation of the labels being carried out by NMR spectroscopy. Both singly and doubly labelled carbon-13 labelled acetate were incorporated into amphotericin $\mathrm{B}$, providing some evidence for the proposed biosynthetic pathway.

A smaller scale extraction process was also formulated which involved solvent extraction followed by purification using HPLC. The detection of incorporated labels into amphotericin for this small scale extraction process was carried out by electrospray mass spectrometry. In the diketide feeding experiment, satisfactory electrospray mass spectra for both amphotericin A and B were obtained, however there was no detection of incorporated labels.

This extraction process was also applied in the search for minor co-metabolites of amphotericin present in the culture broth of $S$. nodosus. Any information gained into 
the possible putative intermediates of amphotericin would hopefully provide evidence for the proposed biosynthetic pathway. Although this initial investigation did not isolate any novel metabolites from the fermentation medium, it did demonstrate the presence of a large number of heptaenes and tetraenes in the extracts at low levels.

Thus, this work has provided some evidence for the proposed processive mode of biosynthesis of amphotericin B. The investigations into the fermentation of Streptomyces nodosus and production of amphotericin B, along with the formulated extraction procedures, has laid the groundwork for any future investigations into the biosynthesis of amphotericin $\mathrm{B}$.

\subsection{Future studies}

Further investigations into the biosynthesis of amphotericin B are likely to involve the use of mutants and genetic studies, as these are the new, exciting areas that may provide a great depth of information. The protocol for high incorporation of acetate into amphotericin B that has been developed in this study will facilitate the determination by NMR of any new analogues that may be produced by these methods. Future studies will build upon this present one in order to provide us with the knowledge to ultimately produce analogues of amphotericin B that are less toxic whilst retaining their life-saving property. 


\section{Clliparer 6}

\section{Experimental}

\subsection{General}

\subsubsection{Materials}

Unless otherwise stated all chemicals were purchased from the Aldrich Chemical Company Ltd, Lancaster Synthesis or Sigma Chemical Company Ltd. All components for the Streptomyces nodosus broth media were either obtained from SmithKline Beecham Pharmaceuticals or purchased from the Sigma Chemical Company Ltd and were the cell tested variety unless otherwise stated. Streptomyces nodosus American type culture collection 14899 was obtained (as a vegetative spore suspension) from SmithKline Beecham Pharmaceuticals, along with the standard sample of amphotericin B.

\subsubsection{Solvents}

Tetrahydrofuran was used dry and was distilled from sodium and benzophenone. Dichloromethane was distilled from calcium hydride. Diisopropylamine was heated at reflux over potassium hydroxide, distilled and stored over molecular sieves. Diethyl 
ether was dried over sodium wire and used without distillation. Petroleum ether (light petroleum) was the $40-60^{\circ} \mathrm{C}$ fraction. Dimethylpyrimidinone (DMPU) was distilled from calcium hydride $\left(88^{\circ} \mathrm{C} / 0.9-1 \mathrm{mbar}\right)$ and stored over molecular sieves. Organic solutions were dried over anhydrous magnesium sulfate and the solvent was removed from reaction mixtures by evaporation under reduced pressure.

\subsubsection{Methods and instrumentation}

Melting points were determined on a Kofler micro heating stage apparatus and are uncorrected. Infra-red spectra were recorded on a Perkin-Elmer 298 spectrophotometer in the specified solvent or as neat films between salt discs. The following abbreviations are used: s-strong, m-medium, w-weak, and br-broad. UV spectra were recorded on a Hewlett Packard 8452A diode array spectrophotometer in the solvent specified using a $1 \mathrm{~cm}$ pathlength. 'H NMR were recorded using a Varian EM390 (90 MHz), Bruker ARX250 (250 MHz) and Bruker AM300 (300 MHz) spectrometers, in the solvent specified with chemical shift values quoted in ppm relative to a TMS internal standard. $J$ Values are quoted in $\mathrm{Hz}$. The following abbreviations were used: s-singlet, d-doublet, t-triplet, q-quartet, m-multiplet and brbroad. ${ }^{13} \mathrm{C}$ NMR were recorded on a Bruker ARX250 (62.9 MHz) and a Bruker AM300 $(75.4 \mathrm{~Hz})$ spectrophotometers in the solvent specified and were proton decoupled, multiplicities being determined by performing DEPT experiments at $90^{\circ}$ and $135^{\circ}$. Specific optical rotation measurements were carried out at room temperature $\left(\mathrm{ca} .22^{\circ} \mathrm{C}\right)$ using a Perkin-Elmer 141 polarimeter in the solvent specified 
and the values are given in $10^{-1} \mathrm{deg}_{\mathrm{cm}^{2}} \mathrm{~g}^{-1}$. Mass spectra were recorded on a Kratos Concept $\mathrm{H}$ double focusing mass spectrometer using either electron impact (EI) at 70 $\mathrm{eV}$ and $170^{\circ} \mathrm{C}$ or electrospray. The $\mathrm{pH}$ of aqueous solutions was measured with a single glass electrode. Flash chromatography was carried out as for Still's method ${ }^{163}$ using Merck Kieselgel 60, 230-400 mesh. Analytical TLC was carried out on commercially prepared plates coated to a thickness of $0.2 \mathrm{~mm}$ with Kieselgel $60 \mathrm{~F}_{254}$. The stains that were used were potassium permanganate and phosphomolybdic acid. High performance liquid chromatography (HPLC) was performed on a Shimadzu LC-4A liquid chromatograph using a Waters Prep Nova Pak® HRC18, $60 \propto 6 \mu \mathrm{m}$, $7.83 \mathrm{~mm}$ column. Adsorption chromatography was carried out using a $2 \times 15 \mathrm{~cm}$ column of diaion HP20 (hydrophobic polyaromatic resin) using a Pharmacia LKB peristaltic pump and a CE $212 \mathrm{~A}$ variable wavelength UV monitor. Data for the Xray crystal structure were collected on a Siemens P4 diffractometer and corrected for Lorentz and polarisation effects but not absorption. The structure was solved by direct methods using the TREF option of the program SHELXTC-PC and refined using full-matrix least-squares using SHELX93. All hydrogen atoms were included in calculated positions $(\mathrm{C}-\mathrm{H}=0.96 \AA$ ) with a fixed isotopic displacement parameter $\left(0.08 \mathrm{e} \AA^{-2}\right)$. The number of atoms refined with anisotropic displacement parameters was limited by the data:parameter ratio. Atoms $\mathrm{C} 19, \mathrm{C} 20$ and $\mathrm{C} 21$ are assigned as partial atoms of some unknown solvent molecule. 


\subsection{Chemical experimentation details}

$(S)$-Vallinol $95^{15}$<smiles>CC(C)=C(N)CO</smiles>

A solution of trimethylsilyl chloride $(8.64 \mathrm{~g}, 80 \mathrm{mmol})$ was added under nitrogen to a solution of lithium borohydride $(0.87 \mathrm{~g}, 40 \mathrm{mmol})$ in of THF $\left(20 \mathrm{~cm}^{3}\right)$ over $2 \mathrm{~min}$, to give a white precipitate of lithium chloride. (S)-Valine $(2.34 \mathrm{~g}, 20 \mathrm{mmol})$ was then added portionwise over $5 \mathrm{~min}$ and the mixture stirred for $24 \mathrm{~h}$ at room temperature. Methanol $\left(30 \mathrm{~cm}^{3}\right)$ was then added cautiously with stirring and the volatiles were removed by distillation (i.e. to dryness) by heating with a mantle at atmospheric pressure. Then potassium hydroxide $\left(20 \% \mathrm{w} / \mathrm{v}, 20 \mathrm{~cm}^{3}\right)$ was added and the organic material extracted with dichloromethane $\left(4 \times 60 \mathrm{~cm}^{3}\right)$. The combined organic extracts were dried, evaporated and purified by Kugelrhör distillation $\left(90-100{ }^{\circ} \mathrm{C}\right.$, $0.1-0.2 \mathrm{mbar}$ ) to give the title compound as a white solid (1.48 $\mathrm{g}, 72 \%)$.

mp 29-30 ${ }^{\circ} \mathrm{C} ;[\alpha]_{\mathrm{D}}+13.1\left(\right.$ ( $\left.1, \mathrm{C}_{2} \mathrm{H}_{5} \mathrm{OH}\right)$, lit. $^{112}[\alpha]_{\mathrm{D}}+14.6$ (neat) (Found: $\mathrm{MH}^{+}$ 104.1075. $\mathrm{C}_{5} \mathrm{H}_{13} \mathrm{NO}$ requires $\mathrm{MH}^{+} 104.1075$ ); $v_{\max .}$ (film) $3350 \mathrm{~s}(\mathrm{br}), 2900 \mathrm{~s}$ (br), 2450s (br), 1600w, 1460s, 1390s, 1370s cm${ }^{-1} ; \delta_{\mathrm{H}}\left(300 \mathrm{MHz} ; \mathrm{CDCl}_{3}\right.$ ) 0.91 ( $3 \mathrm{H}, \mathrm{d}, J$ 6.8, $\left.\mathrm{CH}_{3} \mathrm{CHCH}_{3}\right), 0.92\left(3 \mathrm{H}, \mathrm{d}, J 6.8, \mathrm{CH}_{3} \mathrm{CHCH}_{3}\right), 1.60\left(1 \mathrm{H}, \mathrm{m}, \mathrm{CH}_{3} \mathrm{CHCH}_{3}\right), 2.57$ $\left(1 \mathrm{H}, \mathrm{m}, \mathrm{NH}_{2} \mathrm{CH}\right), 2.64\left(2 \mathrm{H}, \mathrm{br} \mathrm{s}, \mathrm{NH}_{2}\right), 3.31(1 \mathrm{H}, \mathrm{dd}, J 8.6$ and 10.6, $\mathrm{CH} H \mathrm{OH})$, $3.64(1 \mathrm{H}, \mathrm{dd}, J 3.8$ and 10.6, $\mathrm{C} H \mathrm{HOH}) ; \delta_{\mathrm{C}}\left(62.9 \mathrm{MHz} ; \mathrm{CDCl}_{3}\right) 18.42\left(\mathrm{CH}_{3} \mathrm{CHCH}_{3}\right)$, $19.36\left(\mathrm{CH}_{3} \mathrm{CHCH}_{3}\right), 31.26\left(\mathrm{CH}_{3} \mathrm{CHCH}_{3}\right), 58.46\left(\mathrm{NH}_{2} \mathrm{CH}\right), 64.62\left(\mathrm{CH}_{2} \mathrm{OH}\right)$. 
(4S)-4-Isopropyloxazolidin-2-one $9 \mathbb{1}^{105,116}$

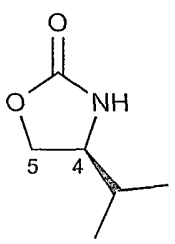

(S)-Valinol $95(1.7 \mathrm{~g}, 17 \mathrm{mmol})$ and diethyl carbonate (1.3 equiv., $\left.2.7 \mathrm{~cm}^{3}, 22 \mathrm{mmol}\right)$ were heated together in toluene $\left(2 \mathrm{~cm}^{3}\right)$ and the toluene was distilled over using a Dean and Stark apparatus under atmospheric pressure (at a temperature of up to 120 $\left.{ }^{\circ} \mathrm{C}\right)$ to azeotrope any water. Sodium methoxide $(0.1$ equiv., $0.1 \mathrm{~g}, 1.7 \mathrm{mmol})$ was added and the mixture heated at reflux for $5 \mathrm{~h}$, occasionally distilling off the ethanol $\left(80-82{ }^{\circ} \mathrm{C}\right)$ that was produced. The reaction mixture was allowed to cool to give a yellow solid which was purified by flash column chromatography $(1: 1$, ethyl acetatelight petroleum; $R_{\mathrm{f}} 0.6$ ) to give the product as a solid which was recrystallised (ethyl acetate and light petroleum) to give white crystals $(1.8 \mathrm{~g}, 82 \%)$.

mp 69-71 ${ }^{\circ} \mathrm{C}$ (lit., $\left.{ }^{99} 71-72{ }^{\circ} \mathrm{C}\right) ;[\alpha]_{\mathrm{D}}+29.0\left(c 1, \mathrm{C}_{2} \mathrm{H}_{5} \mathrm{OH}\right)$ (Found: $\mathrm{M}^{+}, 129.07898$. $\mathrm{C}_{6} \mathrm{H}_{11} \mathrm{NO}_{2}$ requires $\mathrm{M}^{+}, 129.07900$ ); $v_{\text {max. }}$ (nujol) $3300 \mathrm{~s}, 2950 \mathrm{~s}, 1750 \mathrm{~s}, 1480 \mathrm{~m}, 1410 \mathrm{~s}$, $1240 \mathrm{~s} \mathrm{~cm}^{-1} ; \delta_{\mathrm{H}}\left(300 \mathrm{MHz} ; \mathrm{CDCl}_{3}\right) 0.89\left(3 \mathrm{H}, \mathrm{d}, J 6.7, \mathrm{CH}_{3} \mathrm{CHCH}_{3}\right), 0.91(3 \mathrm{H}, \mathrm{d}, J$ 6.8, $\left.\mathrm{CH}_{3} \mathrm{CHCH}_{3}\right), 1.73\left(1 \mathrm{H}, \mathrm{m}, \mathrm{CH}_{3} \mathrm{CHCH}_{3}\right), 3.61(1 \mathrm{H}, \mathrm{m}, 4-H), 4.10(1 \mathrm{H}, \mathrm{dd}, J 6.3$ and 8.7, 5- $\mathrm{HH}), 4.44(1 \mathrm{H}, \mathrm{t}, J 8.7,5-\mathrm{H} H) ; \delta_{\mathrm{C}}\left(62.9 \mathrm{MHz}, \mathrm{CDCl}_{3}\right): 17.7\left(\mathrm{CH}_{3} \mathrm{CHCH}_{3}\right)$, $18.0\left(\mathrm{CH}_{3} \mathrm{CHCH}_{3}\right), 32.7\left(\mathrm{CH}_{3} \mathrm{CHCH}_{3}\right), 58.4(\mathrm{NHCH}), 68.5(\mathrm{C}-5), 160.7(\mathrm{C}-2) ; \mathrm{m} / \mathrm{z}$

(EI) $129\left(\mathrm{M}^{+}, 8.1 \%\right), 86(100), 58(10.5)$. 
(4S)-3-Acetyl-4-isopropyloxazolidin-2-one $96^{99}$

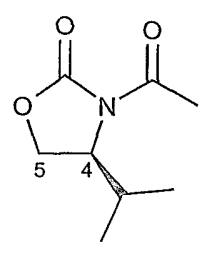

The reaction was carried out under anhydrous conditions. Isopropyloxazolidinone 91 $(0.8 \mathrm{~g}, 6.2 \mathrm{mmol})$ was dissolved in THF $\left(30 \mathrm{~cm}^{3}\right)$ and the solution cooled to $0{ }^{\circ} \mathrm{C}$. To this solution $\mathrm{BuLi}\left(1.6 \mathrm{M}\right.$ in hexane, $3.9 \mathrm{~cm}^{3}, 6.2 \mathrm{mmol}$ ) was added and the reaction stirred for $20 \mathrm{~min}$ before being cooled to $-78^{\circ} \mathrm{C}$. Acetyl chloride (1.15 equiv., 0.5 $\mathrm{cm}^{3}, 7.13 \mathrm{mmol}$ ) was then added and the reaction mixture was stirred at $-78{ }^{\circ} \mathrm{C}$ for 4 h. The reaction was quenched with saturated aqueous ammonium chloride $\left(15 \mathrm{~cm}^{3}\right)$ and extracted with dichloromethane $\left(3 \times 60 \mathrm{~cm}^{3}\right)$. The combined organic extracts were dried, evaporated and purified by flash column chromatography (1:3, ethyl acetate-light petroleum $)$ to give the product $\left(R_{\mathrm{f}} 0.66,1: 1\right.$ ethyl acetate-light petroleum) as a pale yellow oil $(0.79 \mathrm{~g}, 75 \%)$.

(Found: 171.0895. $\mathrm{C}_{8} \mathrm{H}_{13} \mathrm{NO}_{3}$ requires 171.0896); $[\alpha]_{\mathrm{D}}+41.3\left(c \quad 10, \mathrm{CH}_{2} \mathrm{Cl}_{2}\right) ; v_{\text {max. }}$ (film) $2950 \mathrm{~s}, 1790 \mathrm{~s}, 1710 \mathrm{~s}, 1390 \mathrm{~s}, 1250 \mathrm{~s}, 1220 \mathrm{~s} \mathrm{~cm}^{-1} ; \delta_{\mathrm{H}}\left(300 \mathrm{MHz}, \mathrm{CDCl}_{3}\right) 0.88(3$ $\mathrm{H}, \mathrm{d}, J$ 6.9, $\left.\mathrm{CH}_{3} \mathrm{CHCH}_{3}\right), 0.93\left(3 \mathrm{H}, \mathrm{d}, J 7.1, \mathrm{CH}_{3} \mathrm{CHCH}_{3}\right), 2.38(1 \mathrm{H}, \mathrm{m}$, $\left.\mathrm{CH}_{3} \mathrm{CHCH}_{3}\right), 2.50\left(3 \mathrm{H}, \mathrm{s}, \mathrm{COCH}_{3}\right), 4.35(3 \mathrm{H}, \mathrm{m}, 5-\mathrm{HH}$ and $4-H) ; \delta_{\mathrm{C}}(62.9 \mathrm{MHz}$, $\left.\mathrm{CDCl}_{3}\right) 14.9\left(\mathrm{CH}_{3} \mathrm{CHCH}_{3}\right), 18.2\left(\mathrm{CH}_{3} \mathrm{CHCH}_{3}\right), 24.0\left(\mathrm{CH}_{3} \mathrm{CHCH}_{3}\right), 28.9\left(\mathrm{COCH}_{3}\right)$, $58.6(\mathrm{NCH}), 63.6(\mathrm{C}-5), 154.6(\mathrm{C}-2), 170.5\left(\mathrm{C}-1^{\prime}\right) ; m / z(\mathrm{EI}) 171\left(\mathrm{M}^{+}, 29.1 \%\right), 128$ (70.3), $86(100)$. 
(4S)-4-Isopropyl-3-propionyloxazoliding-2-one $92^{99}$

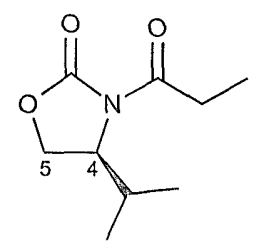

The reaction was carried out under anhydrous conditions. Isopropyloxazolidinone 91 $(2.3 \mathrm{~g}, 18 \mathrm{mmol})$ was dissolved in dry THF $\left(30 \mathrm{~cm}^{3}\right)$ and cooled to $0{ }^{\circ} \mathrm{C}$. BuLi $(1.6 \mathrm{M}$ in hexane, $11.25 \mathrm{~cm}^{3}, 18 \mathrm{mmol}$ ) was added and the solution stirred for $20 \mathrm{~min}$., before being cooled to $-78^{\circ} \mathrm{C}$. Propionyl chloride (1.15 equiv., $1.9 \mathrm{~cm}^{3}, 20.7 \mathrm{mmol}$ ) was added and the reaction stirred at $-78^{\circ} \mathrm{C}$ for $4 \mathrm{~h}$, after which it was quenched with saturated aqueous ammonium chloride $\left(20 \mathrm{~cm}^{3}\right)$ and extracted with dichloromethane $\left(3 \times 60 \mathrm{~cm}^{3}\right)$. The combined extracts were then dried, evaporated and purified by flash column chromatography (1:3, ethyl acetate-light petroleum) or Kugelrhör distillation $\left(114^{\circ} \mathrm{C}, 2.6\right.$ mbar) to give the product $\left(R_{\mathrm{f}} 0.75,1: 1\right.$ ethyl acetate-light petroleum) as a colourless oil $(2.51 \mathrm{~g}, 70 \%)$.

$[\alpha]_{\mathrm{D}}+86.8\left(c 1, \mathrm{CH}_{2} \mathrm{Cl}_{2}\right)$, lit. ${ }^{164}[\alpha]_{\mathrm{D}}+93.0\left(c\right.$ 8.7, $\left.\mathrm{CH}_{2} \mathrm{Cl}_{2}\right)$ Found: 185.1052. $\mathrm{C}_{9} \mathrm{H}_{15} \mathrm{NO}_{3}$ requires 185.1052); $v_{\max }$ (film) 2950s, 1790s, $1710 \mathrm{~s}, 1390 \mathrm{~s}, 1305 \mathrm{~m}$, $1250 \mathrm{~ms}, 1220 \mathrm{~s} \mathrm{~cm}^{-1} ; \delta_{\mathrm{H}}\left(250 \mathrm{MHz} ; \mathrm{CDCl}_{3}\right) 0.88\left(3 \mathrm{H}, \mathrm{d}, J 6.9, \mathrm{CH}_{3} \mathrm{CHCH}_{3}\right), 0.92(3$ $\left.\mathrm{H}, \mathrm{d}, J 7.1, \mathrm{CH}_{3} \mathrm{CHCH}_{3}\right), 1.17\left(3 \mathrm{H}, \mathrm{t}, J 7.4, \mathrm{COCH}_{2} \mathrm{CH}_{3}\right), 2.37\left(1 \mathrm{H}, \mathrm{m}, \mathrm{CH}_{3} \mathrm{CHCH}_{3}\right)$, $2.93\left(2 \mathrm{H}, \mathrm{m}, \mathrm{COCH}_{2} \mathrm{CH}_{3}\right), 4.34(3 \mathrm{H}, \mathrm{m}, 5-\mathrm{HH}$ and $4-\mathrm{H}) ; \delta_{\mathrm{C}}\left(62.9 \mathrm{MHz}, \mathrm{CDCl}_{3}\right) 8.84$ $\left(\mathrm{COCH}_{2} \mathrm{CH}_{3}\right), 15.03\left(\mathrm{CH}_{3} \mathrm{CHCH}_{3}\right), 18.37\left(\mathrm{CH}_{3} \mathrm{CHCH}_{3}\right), 28.76\left(\mathrm{CH}_{3} \mathrm{CHCH}_{3}\right), 29.56$ 
$\left(\mathrm{COCH}_{2} \mathrm{CH}_{3}\right), 58.81(\mathrm{C}-4), 63.77(\mathrm{C}-5), 154.5(\mathrm{C}-2), 174.5\left(\mathrm{C}-1^{\prime}\right) ; m / z(\mathrm{EI}) 185\left(\mathrm{M}^{+}\right.$, 22.6\%), 142 (39.9), 85 (14.9), 57 (100).

(4S)-4-Isopropyl-3-propionyloxazolidin-2-one 92 via $\mathrm{N}$-acetyloxazolidin-2one 96 (in order to prepare labelled material)<smiles>CCC(=O)N1C(=O)OCC1=C(C)C</smiles>

The reaction was carried out under anhydrous conditions. BuLi $(1.6 \mathrm{M}$ in hexane, 1.03 equiv., $6.4 \mathrm{~cm}^{3}, 10.3 \mathrm{mmol}$ ) was added dropwise to solution of DIP (1.1 equiv., $\left.1.54 \mathrm{~cm}^{3}, 11 \mathrm{mmol}\right)$ in THF $\left(10 \mathrm{~cm}^{3}\right)$ a $0^{\circ} \mathrm{C}$ and the mixture was stirred for $20 \mathrm{~min}$. The solution of LDA thus formed was cooled to $-78{ }^{\circ} \mathrm{C}$ and added to a $-78{ }^{\circ} \mathrm{C}$ solution of $96(1.8 \mathrm{~g}, 10 \mathrm{mmol})$ in DMPU and THF $\left(1: 1 \mathrm{v} / \mathrm{v}, 25 \mathrm{~cm}^{3}\right)$ which was then stirred at that temperature for $30 \mathrm{~min}$ in order for the enolate to form. Methyl iodide (3 equiv., $1.86 \mathrm{~cm}^{3}, 30 \mathrm{mmol}$ ) was added dropwise to the mixture which was then stirred for $30 \mathrm{~min}$ at $-78^{\circ} \mathrm{C}$ before being allowed to warm slowly to $0{ }^{\circ} \mathrm{C}$ and then stirred for a further $4 \mathrm{~h}$. The reaction mixture was quenched with hydrochloric acid ( 3 $\left.\mathrm{M}, 20 \mathrm{~cm}^{3}, 50 \mathrm{mmol}\right)$ and extracted with diethyl ether $\left(3 \times 50 \mathrm{~cm}^{3}\right)$. The combined organic extracts were then washed with saturated aqueous sodium hydrogen carbonate $\left(2 \times 30 \mathrm{~cm}^{3}\right)$ and sodium chloride solution $\left(30 \mathrm{~cm}^{3}\right)$, dried, evaporated and purified by flash column chromatography (1:3, ethyl acetate-light petroleum) to give 
the product $\left(R_{\mathrm{f}} 0.75,1: 1\right.$ ethyl acetate-light petroleum) as a colourless oil in $25 \%$ yield (or $32 \%$ based on recovered starting material).

$[\alpha]_{\mathrm{D}}+86.8\left(\mathrm{c} 1, \mathrm{CH}_{2} \mathrm{Cl}_{2}\right)$ (Found: 185.1052. $\mathrm{C}_{9} \mathrm{H}_{15} \mathrm{NO}_{3}$ requires 185.1052); $v_{\text {max. }}$ (film) 2950s, $1790 \mathrm{~s}, 1710 \mathrm{~s}, 1390 \mathrm{~s}, 1305 \mathrm{~m}, 1250 \mathrm{~ms}, 1220 \mathrm{~s} \mathrm{~cm} \mathrm{c}^{-1} ; \delta_{\mathrm{H}}(250 \mathrm{MHz} ;$ $\left.\mathrm{CDCl}_{3}\right) 0.88\left(3 \mathrm{H}, \mathrm{d}, J 6.9, \mathrm{CH}_{3} \mathrm{CHCH}_{3}\right), 0.92\left(3 \mathrm{H}, \mathrm{d}, J 7.05, \mathrm{CH}_{3} \mathrm{CHCH}_{3}\right), 1.17$ (3 $\left.\mathrm{H}, \mathrm{t}, J 7.3, \mathrm{COCH}_{2} \mathrm{CH}_{3}\right), 2.37\left(1 \mathrm{H}, \mathrm{m}, \mathrm{CH}_{3} \mathrm{CHCH}_{3}\right), 2.93\left(1 \mathrm{H}, \mathrm{m}, \mathrm{COCH}_{2} \mathrm{CH}_{3}\right), 4.34$ $(3 \mathrm{H}, \mathrm{m}, 5-\mathrm{HH}$ and $4-\mathrm{H}) ; \delta_{\mathrm{C}}\left(62.9 \mathrm{MHz}, \mathrm{CDCl}_{3}\right) 8.84\left(\mathrm{COCH}_{2} \mathrm{CH}_{3}\right), 15.03$ $\left(\mathrm{CH}_{3} \mathrm{CHCH}_{3}\right), 18.37\left(\mathrm{CH}_{3} \mathrm{CHCH}_{3}\right), 28.76\left(\mathrm{CH}_{3} \mathrm{CHCH}_{3}\right), 29.56\left(\mathrm{COCH}_{2} \mathrm{CH}_{3}\right), 58.81$ (C-4), $\left.63.77(\mathrm{C}-5), 154.5(\mathrm{C}-2), 174.5\left(\mathrm{C}-1^{\prime}\right)^{\prime}\right) ; \mathrm{m} / z(\mathrm{EI}) 185\left(\mathrm{M}^{+}, 22.6 \%\right), 142$ (39.9), 85 (14.9), 57 (100).

$\left(4 S, 2^{\prime} \mathbb{R}\right)-4$-Isopropyl-3-(2'-methyll-3'-oxobutanoyl)oxazolidin-2-one $93^{104}$<smiles>C=C(C(C)=O)C(=O)N1C(=O)OC[C@H]1C(C)=O</smiles>

The reaction was carried out under anhydrous conditions. To a stirred solution of DIP $\left(1.4 \mathrm{~cm}^{3}, 10 \mathrm{mmol}\right)$ in dry THF $\left(20 \mathrm{~cm}^{3}\right)$ at $0^{\circ} \mathrm{C}, \mathrm{BuLi}\left(1.6 \mathrm{M}\right.$ in hexane, $6.25 \mathrm{~cm}^{3}, 10$ mmol) was added and the mixture stirred for $20 \mathrm{~min}$ at $0{ }^{\circ} \mathrm{C}$. The LDA thus formed was then added to a solution of $92(10 \mathrm{mmol})$ in THF $\left(30 \mathrm{~cm}^{3}\right)$ at $0{ }^{\circ} \mathrm{C}$ under nitrogen and the reaction cooled to $-78^{\circ} \mathrm{C}$ and stirred at that temperature for $1.5 \mathrm{~h}$. Acetyl chloride (1.1 equiv., $\left.0.78 \mathrm{~cm}^{3}, 11 \mathrm{mmol}\right)$ in THF $\left(5 \mathrm{~cm}^{3}\right)$ at $0{ }^{\circ} \mathrm{C}$ was added and the 
reaction mixture stirred for $30 \mathrm{~min}$ at $-78^{\circ} \mathrm{C}$ before being quenched with saturated aqueous ammonium chloride $\left(25 \mathrm{~cm}^{3}\right)$ and then diluted with water. The mixture residue was then extracted with dichloromethane $\left(4 \times 60 \mathrm{~cm}^{3}\right)$ and the combined organic extracts were washed with saturated aqueous sodium hydrogen carbonate (2 $\left.\times 30 \mathrm{~cm}^{3}\right)$ and sodium chloride solution $\left(30 \mathrm{~cm}^{3}\right)$, dried and evaporated. The crude product was then purified by flash column chromatography (1:3, ethyl acetate-light petroleum) to give the title compound $\left(R_{\mathrm{f}} 0.59,1: 1\right.$ ethyl acetate-light petroleum) as a white solid which was recrystallised (ethyl acetate and light petroleum) to give white crystals in $57 \%$ yield (or $76 \%$ based on recovered starting material).

mp 131-133 ${ }^{\circ} \mathrm{C}$ (lit., $\left.{ }^{104} 134-135\right) ;[\alpha]_{\mathrm{D}}-18.8\left(c 1, \mathrm{CH}_{2} \mathrm{Cl}_{2}\right)$ (Found: $\mathrm{C}, 58.1 ; \mathrm{H}, 7.6$; $\mathrm{N}, 6.0 . \mathrm{C}_{11} \mathrm{H}_{17} \mathrm{NO}_{4}$ requires $\mathrm{C}, 58.1 ; \mathrm{H}, 7.5 ; \mathrm{N}, 6.2 \%$ ); $v_{\max .}$ (film) $2900 \mathrm{~m}, 1790 \mathrm{~s}$, $1780 \mathrm{~s}, 1720 \mathrm{~s}, 1400 \mathrm{~s}, 1390 \mathrm{~s} \mathrm{~cm}^{-1} ; \delta_{\mathrm{H}}\left(250 \mathrm{MHz} ; \mathrm{CDCl}_{3}\right) 0.92(3 \mathrm{H}, \mathrm{d}, J 6.9$, $\left.\mathrm{CH}_{3} \mathrm{CHCH}_{3}\right), 0.93\left(3 \mathrm{H}, \mathrm{d}, J 7.1, \mathrm{CH}_{3} \mathrm{CHCH}_{3}\right), 1.38\left[3 \mathrm{H}, \mathrm{d}, J 7.3, \mathrm{COCH}\left(\mathrm{CH}_{3}\right) \mathrm{CO}\right.$, $2.32\left(3 \mathrm{H}, \mathrm{s}, \mathrm{COCH}_{3}\right), 2.48\left(1 \mathrm{H}, \mathrm{m}, \mathrm{CH}_{3} \mathrm{CHCH}_{3}\right), 4.35(3 \mathrm{H}, \mathrm{m}, 5-\mathrm{HH}$ and $4-H), 4.50$ $\left(1 \mathrm{H}, \mathrm{q}, J 7.3, \mathrm{COCH}\left(\mathrm{CH}_{3}\right) \mathrm{CO}\right) ; \delta_{\mathrm{C}}\left(62.9 \mathrm{MHz}, \mathrm{CDCl}_{3}\right) 12.74\left[\mathrm{COCH}\left(\mathrm{CH}_{3}\right) \mathrm{CO}\right]$, $14.84\left(\mathrm{CH}_{3} \mathrm{CHCH}_{3}\right), 18.33\left(\mathrm{CH}_{3} \mathrm{CHCH}_{3}\right), 28.66\left(\mathrm{COCH}_{3}\right), 28.70\left(\mathrm{CH}_{3} \mathrm{CHCH}_{3}\right), 53.50$ [COCH$\left(\mathrm{CH}_{3}\right) \mathrm{CO}$ ], 58.98 (C-4), 64.05 (C-5), $154.7(\mathrm{C}-2), 170.12\left(\mathrm{C}-1^{\prime}\right), 205.9\left(\mathrm{C}-3^{\prime}\right)$; $m / z(\mathrm{EI}) 227\left(\mathrm{M}^{+}, 0.9 \%\right), 212(3.3), 185$ (100), 130 (50.1), 99 (35.1), 85 (34.2). 
$\left(2 ' R, 3^{\prime} R, 4 S\right)-4-$ Isopropyl-3-(2'-methyl-3'-hydroxybutanoyl)oxazolidin-2one 99 and $\left(2^{\prime} \mathbb{R}, 3^{\prime} S, 4 S\right)-4$-isopropyl-3-(2'-methyl-3'-hydroxybutanoyl)oxazolidin-2-one 100<smiles>C=C(C)C(=O)N1C(=O)OC[C@H]1C(=O)[C@H](C)O</smiles>

99

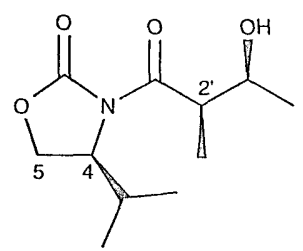

100

Compound $93(0.11 \mathrm{~g}, 0.48 \mathrm{mmol})$ was dissolved in a solvent mixture of methanoldichloromethane $\left(1: 1 \mathrm{v} / \mathrm{v}, 10 \mathrm{~cm}^{3}\right)$ and the solution cooled to $0^{\circ} \mathrm{C}$. Two equivalents of sodium borohydride $(0.04 \mathrm{~g}, 0.96 \mathrm{mmol})$ in methanol-dichloromethane $\left(5 \mathrm{~cm}^{3}\right)$ were added to the reaction mixture which was stirred for $40 \mathrm{~min}$ at $0^{\circ} \mathrm{C}$. The reaction was then quenched with hydrochloric acid $(5 \% \mathrm{v} / \mathrm{v})$ and the organic layer extracted with diethyl ether $\left(3 \times 40 \mathrm{~cm}^{3}\right)$. The combined organic extracts were washed with saturated aqueous sodium hydrogen carbonate $\left(2 \times 20 \mathrm{~cm}^{3}\right)$ then with sodium chloride solution $\left(20 \mathrm{~cm}^{3}\right)$, dried, evaporated and purified by flash column chromatography (1:3, ethyl acetate-light petroleum) to give a pale yellow oil containing the diastereoisomers 99 and $100\left(0.08 \mathrm{~g}, 80 \%\right.$, ratio $1: 2 ; R_{\mathrm{f}} 0.53,1: 1$ ethyl acetate-light petroleum), although structural assignment of the major diastereoisomer was not possible at this stage.

$V_{\max .}\left(\right.$ film) $3500 \mathrm{~s}, 2900 \mathrm{~s}, 1780 \mathrm{~s}, 1690 \mathrm{~s}, 1460 \mathrm{w}, 1390 \mathrm{~s} \mathrm{~cm}^{-1} ; \delta_{\mathrm{H}}\left(250 \mathrm{MHz} ; \mathrm{CDCl}_{3}\right) 0.9$

$\left(3 \mathrm{H}, \mathrm{d}, J 6.9, \mathrm{CH}_{3} \mathrm{CHCH}_{3}\right), 0.94\left(3 \mathrm{H}, \mathrm{d}, J 7.2 \mathrm{CH}_{3} \mathrm{CHCH}_{3}\right), 1.17(3 \mathrm{H}, \mathrm{d}, J$ 7.0, 
$\left[\mathrm{COCH}\left(\mathrm{CH}_{3}\right)\right], 1.21$ [major diastereoisomer: $3 \mathrm{H}, \mathrm{d}, J 6.4, \mathrm{CH}(\mathrm{OH}) \mathrm{CH}_{3}$ ] 1.28 [minor diastereoisomer: $\left.3 \mathrm{H}, \mathrm{d}, J 6.1, \mathrm{CH}(\mathrm{OH}) \mathrm{CH}_{3}\right], 2.39\left(1 \mathrm{H}, \mathrm{m}, \mathrm{CH}_{3} \mathrm{CHCH}_{3}\right), 3.88[1 \mathrm{H}$, $\left.\mathrm{m}, \mathrm{COCH}\left(\mathrm{CH}_{3}\right)\right], 4.16\left[1 \mathrm{H}, \mathrm{m}, \mathrm{CH}(\mathrm{OH}) \mathrm{CH}_{3}\right], 4.35(3 \mathrm{H}, \mathrm{m}, 5-H H$ and $4-H) ; \delta_{\mathrm{C}}(62.9$ $\left.\mathrm{MHz}, \mathrm{CDCl}_{3}\right) \quad 15.1 \quad\left(\mathrm{CH}_{3} \mathrm{CHCH}_{3}\right), \quad 18.4 \quad\left(\mathrm{CH}_{3} \mathrm{CHCH}_{3}\right), 19.7 \quad\left(\mathrm{COCH}_{3}\right), 28.9$ $\left(\mathrm{CH}_{3} \mathrm{CHCH}_{3}\right), 43.0\left(\mathrm{COCHCH}_{3}\right), 59.0(\mathrm{C}-4), 63.8(\mathrm{C}-5), 154.7(\mathrm{C}-2), 176.9\left(\mathrm{C}-1^{\prime}\right)$; $m / z(\mathrm{EI}) 233\left(\mathrm{M}^{+}, 2.4 \%\right), 188(100), 130(45.9), 86(52.7)$.

$\left(2^{\prime} \mathbb{R}, 3^{\prime} \mathbb{R}, 4 S\right)-3-\left[3^{\prime}-\left(3^{\prime \prime}, 5^{\prime \prime}-1 D i n i t r o b e n z o y l o x y\right)-2^{\prime}\right.$-methylloutanoyl]-4-isopropyloxazolidin-2-one 104 and $\left(2^{\prime} \mathbb{R}, 3^{\prime} S, 4, S\right)-3-\left[3^{\prime}-\left(3^{\prime \prime}, 5^{\prime \prime}-\right.\right.$ dinitrobenzoyloxy)-2'-methyllbutanoyl]-4-isopropyloxazolidin-2-one 105<smiles>C=C(C(=O)N1C(=O)OCC1=C(C)C)[C@H](C)OC(=O)c1cc([N+](=O)[O-])cc([N+](=O)[O-])c1</smiles><smiles>CC(C)=C1COC(=O)N1C(=O)C(C)C(C)OC(=O)c1cc([N+](=O)[O-])cc([N+](=O)[O-])c1</smiles>

The mixture of alcohols (99 and 100) $(0.07 \mathrm{~g}, 0.3 \mathrm{mmol})$ were dissolved in dichloromethane $\left(10 \mathrm{~cm}^{3}\right) .3,5-$ Dinitrobenzoyl chloride (2 equiv., $0.14 \mathrm{~g}, 0.6 \mathrm{mmol}$ ) was then added with stirring and the solution turned a yellow colour. Pyridine (2.2 equiv., $0.05 \mathrm{~cm}^{3}, 0.66 \mathrm{mmol}$ ) was next added and the reaction warmed at $c a .30^{\circ} \mathrm{C}$ 
for $2 \mathrm{~h}$. After this time, the reaction mixture was washed with dilute hydrochloric acid and the separated organic phase then washed with sodium hydroxide solution to remove any 3,5-dinitrobenzoic acid. The combined organic phases were dried and evaporated to give the product as two diastereoisomers. Flash column chromatography was used to separate the isomers (5:95, ethyl acetate-toluene), to give $0.02 \mathrm{~g}$ of each isomer $\left(R_{\mathrm{f}} 0.63\right.$ and $0.54,1: 3$ ethyl acetate-toluene) (combined yield including mixed spot $c a .40 \%$ ).

\section{Desired isomer $\left(2^{\prime} R, 3^{\prime} R\right)$}

(Found: 423.1278. $\mathrm{C}_{18} \mathrm{H}_{21} \mathrm{~N}_{3} \mathrm{O}_{9}$ requires 423.1278); $v_{\text {max. }}$ (nujol) 2900m, 1780s, $1740 \mathrm{~s}, 1700 \mathrm{~m}, 1550 \mathrm{~s}, 1380 \mathrm{~m}, 1350 \mathrm{~s} \mathrm{~cm}^{-1} ; \delta_{\mathrm{H}}\left(250 \mathrm{MHz}, \mathrm{CDCl}_{3}\right) 0.74(3 \mathrm{H}, \mathrm{d}, J 7.0$, $\left.\mathrm{CH}_{3} \mathrm{CHCH}_{3}\right), 0.82\left(3 \mathrm{H}, \mathrm{d}, \quad J \quad 7.0, \mathrm{CH}_{3} \mathrm{CHCH}_{3}\right), \quad 1.29[3 \mathrm{H}, \mathrm{d}, J \quad 6.9$, $\left.\mathrm{COCH}\left(\mathrm{CH}_{3}\right) \mathrm{CHO}\left(\mathrm{CH}_{3}\right)\right], 1.48\left[3 \mathrm{H}, \mathrm{d}, J 6.4, \mathrm{COCH}\left(\mathrm{CH}_{3}\right) \mathrm{CHO}(\mathrm{CH})_{3}\right], 2.18(1 \mathrm{H}, \mathrm{m}$, $\left.\mathrm{CH}_{3} \mathrm{CHCH}_{3}\right), 4.30\left[4 \mathrm{H}, \mathrm{m}, \mathrm{COCH}\left(\mathrm{CH}_{3}\right) \mathrm{CHO}\left(\mathrm{CH}_{3}\right), 5-\mathrm{HH}\right.$ and $\left.4-H\right], 5.58[1 \mathrm{H}, \mathrm{dq}$, $J 6.9$ and 2.0, $\left.\mathrm{COCH}\left(\mathrm{CH}_{3}\right) \mathrm{CHO}\left(\mathrm{CH}_{3}\right)\right], 9.16\left(3 \mathrm{H}, \mathrm{m}, \mathrm{C}_{6} \mathrm{H}_{3} \mathrm{~N}_{2} \mathrm{O}_{4}\right) ; \delta_{\mathrm{C}}(62.9 \mathrm{MHz}$, $\left.\mathrm{CDCl}_{3}\right) 10.57\left[\mathrm{COCH}\left(\mathrm{CH}_{3}\right) \mathrm{CHO}\left(\mathrm{CH}_{3}\right)\right], 14.84\left(\mathrm{CH}_{3} \mathrm{CHCH}_{3}\right), 18.32\left(\mathrm{CH}_{3} \mathrm{CHCH}_{3}\right)$, $18.41\left[\mathrm{COCH}\left(\mathrm{CH}_{3}\right) \mathrm{CHO}\left(\mathrm{CH}_{3}\right)\right], 28.70 \quad\left(\mathrm{CH}_{3} \mathrm{CHCH}_{3}\right), \quad 42.06 \quad\left[\mathrm{COCH}\left(\mathrm{CH}_{3}\right)-\right.$ $\left.\mathrm{CHO}\left(\mathrm{CH}_{3}\right)\right], 58.98(\mathrm{C}-4), 63.8(\mathrm{C}-5), 122.76,125.27,128.56,129.42,129.84,134.55$ (Ph C-1), $149.08\left[\mathrm{COCH}\left(\mathrm{CH}_{3}\right) \mathrm{CHO}\left(\mathrm{CH}_{3}\right)\right], 154.65(\mathrm{C}-2), 162.31(\mathrm{COPh}), 172.67$ $\left[\mathrm{COCH}\left(\mathrm{CH}_{3}\right) \mathrm{CHO}\left(\mathrm{CH}_{3}\right)\right] ; \mathrm{m} / z(\mathrm{EI}) 423\left(\mathrm{M}^{+}, 1.4 \%\right), 295(48.3), 211(100), 196$ (95.6), 83 (89.3). 


\section{Control isomer $\left(2^{\prime} \mathbb{R}, 3 ' S\right)$}

(Found: 423.1278. $\quad \mathrm{C}_{18} \mathrm{H}_{21} \mathrm{~N}_{3} \mathrm{O}_{9}$ requires 423.1278); $v_{\text {max. }}$ (nujol) 2900s, 1780s, $1740 \mathrm{~m}, 1700 \mathrm{~m}, 1550 \mathrm{~s}, 1350 \mathrm{~s} \mathrm{~cm}^{-1} ; \delta_{\mathrm{H}}\left(250 \mathrm{MHz}, \mathrm{CDCl}_{3}\right) 0.91(3 \mathrm{H}, \mathrm{d}, J 7.0$, $\left.\mathrm{CH}_{3} \mathrm{CHCH}_{3}\right), 0.93\left(3 \mathrm{H}, \mathrm{d}, J 6.8, \mathrm{CH}_{3} \mathrm{CHCH}_{3}\right), 1.31$ [3 H, d, $J$ 6.9, $\mathrm{COCH}\left(\mathrm{CH}_{3}\right)-$ $\left.\mathrm{CHO}\left(\mathrm{CH}_{3}\right)\right], 1.49\left[3 \mathrm{H}, \mathrm{d}, J\right.$ 6.4, $\left.\mathrm{COCH}\left(\mathrm{CH}_{3}\right) \mathrm{CHO}\left(\mathrm{CH}_{3}\right)\right], 2.31(1 \mathrm{H}, \mathrm{m}$, $\left.\mathrm{CH}_{3} \mathrm{CHCH}_{3}\right), 4.32\left[1 \mathrm{H}, \mathrm{dq}, J 6.4\right.$ and $\left.2.9, \mathrm{COCH}\left(\mathrm{CH}_{3}\right) \mathrm{CHO}\left(\mathrm{CH}_{3}\right)\right], 4.35(3 \mathrm{H}, \mathrm{m}, 5-$ $H H$ and 4-H), $5.65\left[1 \mathrm{H}, \mathrm{dq}, J 6.9\right.$ and $\left.2.5, \mathrm{COCH}\left(\mathrm{CH}_{3}\right) \mathrm{CHO}\left(\mathrm{CH}_{3}\right)\right], 9.18(3 \mathrm{H}, \mathrm{m}$, $\left.\mathrm{C}_{6} \mathrm{H}_{3} \mathrm{~N}_{2} \mathrm{O}_{4}\right) ; \quad \delta_{\mathrm{C}}\left(62.9 \quad \mathrm{MHz}, \quad \mathrm{CDCl}_{3}\right) \quad 10.57 \quad\left[\mathrm{COCH}\left(\mathrm{CH}_{3}\right) \mathrm{CHO}\left(\mathrm{CH}_{3}\right)\right], \quad 14.84$ $\left(\mathrm{CH}_{3} \mathrm{CHCH}_{3}\right), 18.37\left[\mathrm{CH}_{3} \mathrm{CHCH}_{3}\right.$ and $\left.\mathrm{COCH}\left(\mathrm{CH}_{3}\right) \mathrm{CHO}\left(\mathrm{CH}_{3}\right)\right], 28.70\left(\mathrm{CH}_{3} \mathrm{CHCH}_{3}\right)$, $42.06\left[\mathrm{COCH}\left(\mathrm{CH}_{3}\right) \mathrm{CHO}\left(\mathrm{CH}_{3}\right)\right], 58.98$ (C-4), 63.8 (C-5), 122.76, 125.27, 128.56, 129.42, 129.84, 134.55 ( $\mathrm{Ph} \mathrm{C}-1), 149.08\left[\mathrm{COCH}\left(\mathrm{CH}_{3}\right) \mathrm{CHO}\left(\mathrm{CH}_{3}\right)\right], 154.65(\mathrm{C}-2)$, $162.31(\mathrm{COPh}), 172.67\left[\mathrm{COCH}\left(\mathrm{CH}_{3}\right) \mathrm{CHO}\left(\mathrm{CH}_{3}\right)\right] ; \mathrm{m} / \mathrm{z}(\mathrm{EI}) 423\left(\mathrm{M}^{+}, 1.0 \%\right), 295$ (53.2), 211 (89.4), $196(100), 83$ (82.1).

$(2 \mathbb{R}, 3 S)-3-H-H y d r o x y-2-m e t h y l b u t a n o i c$ acid 109 (not isolated) $)^{138}$<smiles>C=C(C(C)=O)C(=O)O</smiles> 
To a solution of the dinitrobenzoate $\mathbf{1 0 5}(90 \mathrm{mg}, 0.213 \mathrm{mmol})$ in THF-water $(4: 1,16$ $\left.\mathrm{cm}^{3}\right)$ under nitrogen, hydrogen peroxide $\left(30 \% \mathrm{v} / \mathrm{v}, 0.18 \mathrm{~cm}^{3} 1.74 \mathrm{mmol}\right)$ and lithium hydroxide $(34 \mathrm{mg}, 0.852 \mathrm{mmol})$ in water $\left(7 \mathrm{~cm}^{3}\right)$ were added. The reaction mixture was stirred at $0{ }^{\circ} \mathrm{C}$ for $6 \mathrm{~h}$, then quenched with sodium sulfite $(200 \mathrm{mg}, 0.74 \mathrm{mmol})$ in water $\left(8 \mathrm{~cm}^{3}\right)$. The THF was removed in vacuo and the resulting mixture was extracted with dichloromethane $\left(4 \times 20 \mathrm{~cm}^{3}\right)$ to remove the chiral auxiliary 91 which was recovered in $74 \%$ yield. The aqueous layer was acidified to $\mathrm{pH} 1$ with dilute hydrochloric acid and saturated sodium chloride solution was added (to assist in the extraction of acids). The acids were then extracted with ethyl acetate $\left(5 \times 20 \mathrm{~cm}^{3}\right)$, the combined organic extracts dried and evaporated to give a mixture of the title compound 109 and the by-product, dinitrobenzoic acid 110 , of combined weight 33 $\mathrm{mg}(47 \%)$.

$\delta_{\mathrm{H}}\left(90 \mathrm{MHz}, \mathrm{CDCl}_{3}\right)$ 109: $1.25\left[6 \mathrm{H}, 2 \times \mathrm{d}, \mathrm{COCH}\left(\mathrm{CH}_{3}\right) \mathrm{CH}(\mathrm{OH}) \mathrm{CH}_{3}\right], 2.45[1 \mathrm{H}, \mathrm{m}$, $\left.\mathrm{COCH}\left(\mathrm{CH}_{3}\right)\right], 3.80\left[1 \mathrm{H}, \mathrm{m}, \mathrm{CH}(\mathrm{OH}) \mathrm{CH}_{3}\right] ; 110: 9.20\left(3 \mathrm{H}, \mathrm{m}, \mathrm{C}_{6} \mathrm{H}_{3} \mathrm{~N}_{2} \mathrm{O}_{4}\right)$.

\section{Ethyl 3-hydroxy-2-methylbutanoate 116}<smiles>CCOC(=O)C(C)C(C)O</smiles>

To a solution of ethyl methylacetoacetate $115(3 \mathrm{~g}, 0.02 \mathrm{M})$ in methanol $\left(50 \mathrm{~cm}^{3}\right)$ at 0

${ }^{\circ} \mathrm{C}$, sodium borohydride $(0.75 \mathrm{~g}, 0.02 \mathrm{M})$ was added. The reaction mixture was 
stirred at $0{ }^{\circ} \mathrm{C}$ for $2 \mathrm{~h}$ before being quenched with hydrochloric acid $(25 \% \mathrm{v} / \mathrm{v}, 25$ $\left.\mathrm{cm}^{3}\right)$. The solution was then extracted with diethyl ether $\left(3 \times 30 \mathrm{~cm}^{3}\right)$, the combined ether layers washed with saturated aqueous sodium hydrogen carbonate then saturated sodium chloride solution, dried and evaporated to give the product as a pale cream oil $(3.7 \mathrm{~g}, 80 \%)$

$\delta_{\mathrm{H}}\left(90 \mathrm{MHz}, \mathrm{CDCl}_{3}\right) 1.19\left[3 \mathrm{H}, \mathrm{d}, J 6.3, \mathrm{CH}(\mathrm{OH}) \mathrm{CH}_{3}\right], 1.2(3 \mathrm{H}, \mathrm{d}, J 6.3$, $\left.\operatorname{COCH}\left(\mathrm{CH}_{3}\right)\right], 1.21\left(3 \mathrm{H}, \mathrm{t}, J 6.3, \mathrm{COOCH}_{2} \mathrm{CH}_{3}\right), 2.4\left[1 \mathrm{H}, \mathrm{m}, \mathrm{COCH}\left(\mathrm{CH}_{3}\right)\right], 3.8[1$ $\left.\mathrm{H}, \mathrm{m}, \mathrm{CH}(\mathrm{OH}) \mathrm{CH}_{3}\right], 4.1\left(2 \mathrm{H}, \mathrm{q}, J 6.3, \mathrm{COOCH}_{2} \mathrm{CH}_{3}\right)$.

\section{3-Hydroxy-2-methylbutanoic acid 117}<smiles>CC(O)C(C)C(=O)O</smiles>

To a solution of the ester $116(1.2 \mathrm{~g}, 8.2 \mathrm{mmol})$ in $\mathrm{THF}-\mathrm{H}_{2} \mathrm{O}\left(3: 1,20 \mathrm{~cm}^{3}\right)$, sodium hydroxide $(0.7 \mathrm{~g}, 16.4 \mathrm{mmol})$ in $\mathrm{H}_{2} \mathrm{O}\left(15 \mathrm{~cm}^{3}\right)$ was added and the reaction warmed to ca. $30^{\circ} \mathrm{C}$ for $1.5 \mathrm{~h}$. After this time the THF was removed in vacuo and the resulting mixture was extracted with dichloromethane $\left(3 \times 30 \mathrm{~cm}^{3}\right)$. The aqueous layer was then acidified to $\mathrm{pH} 1$ with dilute hydrochloric acid and extracted with ethyl acetate $\left(3 \times 30 \mathrm{~cm}^{3}\right)$. The combined ethyl acetate extracts were then dried and evaporated to give the product as a pale yellow oil $(0.6 \mathrm{~g}, 63 \%)$.

$\delta_{\mathrm{H}}\left(90 \mathrm{MHz}, \mathrm{CDCl}_{3}\right) 1.25\left[6 \mathrm{H}, 2 \times \mathrm{d}, J 6.3, \mathrm{COCH}\left(\mathrm{CH}_{3}\right) \mathrm{CH}(\mathrm{OH}) \mathrm{CH}_{3}\right], 2.5[1 \mathrm{H}, \mathrm{m}$, $\left.\mathrm{COCH}\left(\mathrm{CH}_{3}\right)\right], 4.0\left[1 \mathrm{H}, \mathrm{m}, \mathrm{CH}(\mathrm{OH}) \mathrm{CH}_{3}\right], 7.4(2 \mathrm{H}, \mathrm{br} \mathrm{s}, 2 \times \mathrm{OH})$. 
$N$-Acetylcysteamine [N-(3-sulfanyl)propanylacetamide $] 112^{1,34}$<smiles>CC(=O)NCCS</smiles>

A solution of $N, S$-diacetylcysteamine $\mathbf{1 1 1}$ (previously prepared in our group following the method in ref. 139) $(0.1 \mathrm{~g}, 0.6 \mathrm{mmol})$ in degassed $\mathrm{H}_{2} \mathrm{O}\left(5 \mathrm{~cm}^{3}\right)$ was cooled to $0{ }^{\circ} \mathrm{C}$. Potassium hydroxide $(0.11 \mathrm{~g}, 2.04 \mathrm{mmol})$ was added and the reaction mixture stirred for $30 \mathrm{~min}$ at room temperature. The clear solution was then adjusted to $\mathrm{pH} 7$ with dilute hydrochloric acid and saturated sodium chloride solution was added. The product was extracted with dichloromethane $\left(4 \times 30 \mathrm{~cm}^{3}\right)$, and the combined organic extracts were dried and evaporated to give the product as a colourless oil $(70 \mathrm{mg}, 95 \%)$. The product decomposes rapidly and was therefore used immediately in the thioesterification reactions.

$V_{\max }\left(\right.$ film) $3300 \mathrm{~s}, 3050 \mathrm{~m}, 2900 \mathrm{~m}, 2550 \mathrm{w}, 1660 \mathrm{~s}, 1550 \mathrm{~s}, 1430 \mathrm{~m} \mathrm{~cm}^{-1} ; \delta_{\mathrm{H}}(300 \mathrm{MHz}$, $\left.\mathrm{CDCl}_{3}\right) 1.45(1 \mathrm{H}, \mathrm{t}, J 8.4, \mathrm{SH}), 2.0\left(3 \mathrm{H}, \mathrm{s}, \mathrm{CH}_{3} \mathrm{CO}\right), 2.65\left(2 \mathrm{H}, \mathrm{m}, \mathrm{CH}_{2} \mathrm{SH}\right), 3.4(2$ $\left.\mathrm{H}, \mathrm{m}, \mathrm{CH}_{2} \mathrm{NH}\right), 6.7(1 \mathrm{H}$, br s, $\mathrm{NH})$. 
3-hydroxy-2-methylbutanoyl $N$-acetylcysteamine [S-2-(acetylamino)ethyl 3-hydroxy-2-methylbutanethioate] $118^{140,141}$<smiles>CC(=O)NCCSC(=O)C(C)C(C)O</smiles>

To a solution of the acid $117(320 \mathrm{mg}, 2.7 \mathrm{mmol})$ in dichloromethane $\left(50 \mathrm{~cm}^{3}\right)$ at 0 ${ }^{\circ} \mathrm{C}$, DMAP (33 mg, $\left.0.27 \mathrm{mmol}\right), N$-acetylcysteamine $(0.32 \mathrm{~g}, 2.7 \mathrm{mmol})$ and DCC $(0.84 \mathrm{~g}, 4.05 \mathrm{mmol})$ were added and the reaction mixture stirred for 10 minutes at 0 ${ }^{\circ} \mathrm{C}$ and then for $18 \mathrm{~h}$ at room temperature. The solution was filtered through Celite and the Celite washed with ethyl acetate. The filtrate was evaporated to give a crude mixture containing excess $N$-acetylcysteamine $\left(R_{\mathrm{f}} \quad 0.32\right)$ and some residual dicyclohexylurea $\left(R_{\mathrm{f}} 0.73\right)$, and was purified by flash chromatography (ethyl acetate) to give the title thioester $\left(0.17 \mathrm{~g}, 33 \%, R_{\mathrm{f}} 0.19\right)$.

(Found 219.0929. $\mathrm{C}_{11} \mathrm{H}_{14} \mathrm{NO}_{3}$ requires 219.0929); $v_{\max }$ (film) 3300s (br), 2950s, $1660 \mathrm{~s}, 1550 \mathrm{~s}, 1460 \mathrm{~m} \mathrm{~cm}^{-1} ; \delta_{\mathrm{H}}\left(300 \mathrm{MHz}, \mathrm{CDCl}_{3}\right) 1.18\left[3 \mathrm{H}, \mathrm{d}, J 7.2, \mathrm{COCH}\left(\mathrm{CH}_{3}\right)\right]$, $1.22\left[3 \mathrm{H}, \mathrm{d}, J 6.3, \mathrm{CH}(\mathrm{OH}) \mathrm{CH}_{3}\right], 1.96\left(3 \mathrm{H}, \mathrm{s}, \mathrm{CH}_{3} \mathrm{CONH}\right), 2.7[1 \mathrm{H}, \mathrm{dq}, J 7.1$ and 3.6, $\left.\mathrm{COCH}\left(\mathrm{CH}_{3}\right)\right], 3.03\left(2 \mathrm{H}, \mathrm{m}, \mathrm{CH}_{2} \mathrm{~S}\right), 3.42\left(2 \mathrm{H}, \mathrm{m}, \mathrm{CH}_{2} \mathrm{NH}\right), 4.02[1 \mathrm{H}, \mathrm{m}$, $\left.\mathrm{CH}(\mathrm{OH}) \mathrm{CH}_{3}\right], 6.74(1 \mathrm{H}$, br s, $\mathrm{N} H) ; \delta_{\mathrm{C}}\left(62.9 \mathrm{MHz}, \mathrm{CDCl}_{3}\right) 15.0\left[\mathrm{COCH}\left(\mathrm{CH}_{3}\right)\right], 21.2$ [CH(OH) $\left.\mathrm{CH}_{3}\right], 23.4\left(\mathrm{CH}_{3} \mathrm{CONH}\right), 28.9\left(\mathrm{CH}_{2} \mathrm{~S}\right), 39.5\left(\mathrm{CH}_{2} \mathrm{NH}\right), 56.5\left[\mathrm{COCH}\left(\mathrm{CH}_{3}\right)\right]$, $70.1\left[\mathrm{CH}(\mathrm{OH}) \mathrm{CH}_{3}\right], 171.5\left(\mathrm{CH}_{2} \mathrm{CONH}\right), 204.0(\mathrm{COS}) ; \mathrm{m} / \mathrm{z}(\mathrm{EI}) 219\left(\mathrm{M}^{+}, 2.1 \%\right), 145$ (46.5), 119 (100), 60 (69.7). 
(2R,3S)-3-Hydroxy-2-methylbutanoyl $N$-acetylcysteamine $\quad\{S$-2-(acetyl amino)ethyl $(2 \mathbb{R}, 3 S)-3-$ hhydroxy $-2-\left[{ }^{2} \mathbb{H}_{3}\right]$ methyl[ $\left.3-^{2} \mathbb{H}\right]$ butanethioate 113<smiles>C=C(C(=O)SCCNC(C)=O)C(C)O</smiles>

DMAP (2 $\mathrm{mg}, 0.016 \mathrm{mmol})$ and $N$-acetylcysteamine $(20 \mathrm{mg}, 0.164 \mathrm{mmol}$ ) were added to a solution of the acids 109 and $110(33 \mathrm{mg}, 0.16 \mathrm{mmol})$ in dichloromethane $\left(7 \mathrm{~cm}^{3}\right)$ at $0^{\circ} \mathrm{C}$. DCC (50 mg, $\left.0.24 \mathrm{mmol}\right)$ was added and the solution was stirred for $10 \mathrm{~min}$ at $0^{\circ} \mathrm{C}$ and then for a further $18 \mathrm{~h}$ at room temperature. The solution was then filtered through Celite and the Celite washed with ethyl acetate. The filtrate was evaporated to give an oil containing residual dicyclohexylurea (TLC system: acetone-toluene, $\left.1: 1 ; R_{\mathrm{f}} 0.73\right)$, the dinitrobenzoate thioester $\left(R_{\mathrm{f}} 0.48\right)$, excess $N$ acetylcysteamine $\left(R_{\mathrm{f}} 0.36\right)$ and the product thioester $\left(R_{\mathrm{f}} 0.2\right)$, and was separated by flash chromatography (1:2, acetone-toluene) to give the title compound as an oil (8 $\mathrm{mg}, 41 \%$, ).

(Found 219.0929. $\mathrm{C}_{11} \mathrm{H}_{14} \mathrm{NO}_{3}$ requires 219.0929); $v_{\max .}$ (film) 3300s (br), 2950s, $1660 \mathrm{~s}, 1550 \mathrm{~s}, 1460 \mathrm{~m} \mathrm{~cm}^{-1} ; \delta_{\mathrm{H}}\left(300 \mathrm{MHz}, \mathrm{CDCl}_{3}\right) 1.22\left[3 \mathrm{H}, \mathrm{d}, J 7.0, \mathrm{COCH}\left(\mathrm{CH}_{3}\right)\right]$, $1.24\left[3 \mathrm{H}, \mathrm{d}, J 6.2, \mathrm{CH}(\mathrm{OH}) \mathrm{CH}_{3}\right], 1.97\left(3 \mathrm{H}, \mathrm{s}, \mathrm{CH}_{3} \mathrm{CONH}\right), 2.69[1 \mathrm{H}, \mathrm{dq}, J 7.1$ and 3.4, $\left.\mathrm{COCH}\left(\mathrm{CH}_{3}\right)\right], 3.05\left(2 \mathrm{H}, \mathrm{m}, \mathrm{CH}_{2} \mathrm{~S}\right), 3.46\left(2 \mathrm{H}, \mathrm{m}, \mathrm{CH}_{2} \mathrm{NH}\right), 3.95[1 \mathrm{H}, \mathrm{m}$, $\left.\mathrm{CH}(\mathrm{OH}) \mathrm{CH}_{3}\right] ; \delta_{\mathrm{C}}\left(62.9 \mathrm{MHz}, \mathrm{CDCl}_{3}\right) 15.1\left[\mathrm{COCH}\left(\mathrm{CH}_{3}\right)\right], 21.2\left[\mathrm{CH}(\mathrm{OH}) \mathrm{CH}_{3}\right], 23.5$ $\left(\mathrm{CH}_{3} \mathrm{CONH}\right), 29.0\left(\mathrm{CH}_{2} \mathrm{~S}\right), 39.3\left(\mathrm{CH}_{2} \mathrm{NH}\right), 56.5\left[\mathrm{COCH}\left(\mathrm{CH}_{3}\right)\right], 70.0\left[\mathrm{CH}(\mathrm{OH}) \mathrm{CH}_{3}\right]$, 
$171.4\left(\mathrm{CH}_{3} \mathrm{CONH}\right), 203.9(\mathrm{COS}) ; \mathrm{m} / z$ (EI) $219\left(\mathrm{M}^{+}, 2.1 \%\right), 145$ (46.5), 119 (100), 60 (69.7).

\section{$(R)$-Valinol $120^{115}$}<smiles>CC(C)[C@H](N)CO</smiles>

A solution of trimethylsilyl chloride $(82.6 \mathrm{~g}, 0.76 \mathrm{~mol})$ was added under nitrogen to a solution of lithium borohydride $(8.28 \mathrm{~g}, 0.38 \mathrm{~mol})$ in THF $\left(150 \mathrm{~cm}^{3}\right)$ over a period of twenty minutes, during which time a precipitate of lithium chloride formed. $(R)$ Valine 119 (22 $\mathrm{g}, 0.19 \mathrm{~mol})$ was then added portionwise over $45 \mathrm{~min}$ and the reaction mixture stirred at room temperature for $24 \mathrm{~h}$. Methanol $\left(62.9 \mathrm{~cm}^{3}\right)$ was added cautiously and the volatiles were removed by distillation (i.e. to dryness) by heating with a mantle at atmospheric pressure.. Potassium hydroxide solution $(20 \% \mathrm{w} / \mathrm{v}, 200$ $\mathrm{cm}^{3}$ ) was then added to the stirred residue, and the mixture then extracted with dichloromethane $\left(4 \times 200 \mathrm{~cm}^{3}\right)$. The combined organic extracts were then dried, evaporated and purified by Kugelrhör distillation $\left(90-100^{\circ} \mathrm{C}, 0.1-0.2 \mathrm{mbar}\right)$ to give the title compound $(15.23 \mathrm{~g}, 78 \%)$.

$[\alpha]_{\mathrm{D}}-15.2\left(c 1, \mathrm{C}_{2} \mathrm{H}_{5} \mathrm{OH}\right)$, lit. ${ }^{165}[\alpha]_{\mathrm{D}}-16.0\left(c 10, \mathrm{C}_{2} \mathrm{H}_{5} \mathrm{OH}\right)$ (Found: $\mathrm{MH}^{+}$104.1075. $\mathrm{C}_{5} \mathrm{H}_{13} \mathrm{NO}$ requires $\mathrm{MH}^{+} 104.1075$ ); $v_{\text {max. }}$ (nujol) $3350 \mathrm{~s}$ (br), 2900s, 2450s, $1600 \mathrm{w}$, $1460 \mathrm{~s}, 1390 \mathrm{~s}, 1370 \mathrm{~s} \mathrm{~cm}^{-1} ; \delta_{\mathrm{H}}\left(250 \mathrm{MHz}, \mathrm{CDCl}_{3}\right) 0.91\left(6 \mathrm{H}, \mathrm{d}, J 6.8, \mathrm{CH}_{3} \mathrm{CHCH}_{3}\right)$, $0.92\left(6 \mathrm{H}, \mathrm{d}, J 6.8, \mathrm{CH}_{3} \mathrm{CHCH}_{3}\right), 1.60\left(1 \mathrm{H}, \mathrm{m}, \mathrm{CH}_{3} \mathrm{CHCH}_{3}\right), 2.55\left(1 \mathrm{H}, \mathrm{m}, \mathrm{NH}_{2} \mathrm{CH}\right)$, $2.65\left(2 \mathrm{H}\right.$, br s, $\left.N H_{2}\right), 3.30(1 \mathrm{H}, \mathrm{dd}, J 8.4$ and 10.6, $\mathrm{CH} H \mathrm{OH}), 3.65(1 \mathrm{H}$, dd, $J 3.8$ 
and 10.6, $\mathrm{CHHOH}) ; \delta_{\mathrm{C}}\left(62.9 \mathrm{MHz}, \mathrm{CDCl}_{3}\right) 18.42\left(\mathrm{CH}_{3} \mathrm{CHCH}_{3}\right), 19.38\left(\mathrm{CH}_{3} \mathrm{CHCH}_{3}\right)$, $31.04\left(\mathrm{CH}_{3} \mathrm{CHCH}_{3}\right), 58.45(\mathrm{CHOH}), 64.48\left(\mathrm{CHNH}_{2}\right)$.

\section{(4R)-4-Isopropyloxazolidin-2-one $121^{105,116}$}

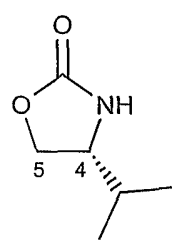

(R)-Valinol $120(11.5 \mathrm{~g}, 0.112 \mathrm{~mol})$ and diethyl carbonate $\left(17.6 \mathrm{~cm}^{3}, 0.145 \mathrm{~mol}\right)$, were heated together in toluene $\left(10 \mathrm{~cm}^{3}\right)$ and the toluene was distilled over to azeotrope any water using a Dean and Stark apparatus at atmospheric pressure (with heating up to $\left.120^{\circ} \mathrm{C}\right)$. Sodium methoxide $(0.61 \mathrm{~g}, 0.0112 \mathrm{~mol})$ was added and the mixture heated under reflux for $5 \mathrm{~h}$, occasionally distilling off the ethanol that was produced. The reaction mixture was allowed to cool to give a yellow solid which was purified by flash column chromatography (1:1, ethyl acetate-light petroleum; $R_{\mathrm{f}}$ 0.6) to give the product as a solid which was recrystallised (ethyl acetate and light petroleum) to give white crystals $(12.12 \mathrm{~g}, 84 \%)$.

mp $70{ }^{\circ} \mathrm{C}$ (lit., $\left.{ }^{99} 71-72{ }^{\circ} \mathrm{C}\right) ;[\alpha]_{\mathrm{D}}-23.6\left(c 1, \mathrm{C}_{2} \mathrm{H}_{5} \mathrm{OH}\right)$ (Found: $\mathrm{C}, 55.7 ; \mathrm{H}, 8.6 ; \mathrm{N}$, 10.9. $\mathrm{C}_{6} \mathrm{H}_{11} \mathrm{NO}_{2}$ requires $\mathrm{C}, 55.8 ; \mathrm{H}, 8.6 ; \mathrm{N}, 10.8 \%$ ); $v_{\text {max. }}$ (nujol) $3629 \mathrm{~s}, 2900 \mathrm{~s}$, $1750 \mathrm{~s}, 1480 \mathrm{~m}, 1405 \mathrm{~s}, 1240 \mathrm{~s} \mathrm{~cm}^{-1} ; \delta_{\mathrm{H}}\left(250 \mathrm{MHz}, \mathrm{CDCl}_{3}\right) 0.90(3 \mathrm{H}, \mathrm{d}, J 6.8$, $\left.\mathrm{CH}_{3} \mathrm{CHCH}_{3}\right), 0.95\left(3 \mathrm{H}, \mathrm{d}, J 6.7, \mathrm{CH}_{3} \mathrm{CHCH}_{3}\right), 1.73\left(1 \mathrm{H}, \mathrm{m}, \mathrm{CH}_{3} \mathrm{CHCH}_{3}\right), 3.63(1 \mathrm{H}$, $\mathrm{m}, 4-H), 4.10\left(1 \mathrm{H}\right.$, dd, $J 6.3$ and 8.7, 5-HH), $4.45(1 \mathrm{H}, \mathrm{t}, J 8.7,5-\mathrm{H} H) ; \delta_{\mathrm{C}}(62.9$ 
$\left.\mathrm{MHz}, \mathrm{CDCl}_{3}\right) 17.7\left(\mathrm{CH}_{3} \mathrm{CHCH}_{3}\right), 18.0\left(\mathrm{CH}_{3} \mathrm{CHCH}_{3}\right), 32.7\left(\mathrm{CH}_{3} \mathrm{CHCH}_{3}\right), 58.4(\mathrm{C}-$ 4), $68.7(\mathrm{C}-5), 160.7(\mathrm{C}-2) ; \mathrm{m} / \mathrm{z}$ (EI) $129\left(\mathrm{M}^{+}, 8.3 \%\right), 86(100), 58(10.4)$.

\section{(4R)-3-acetyl-4-isopropyloxazolidin-2-one 122}

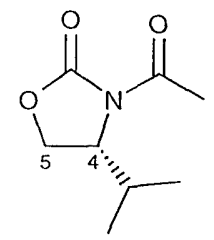

Oxazolidinone 121 (12 g, $0.09 \mathrm{~mol})$ was dissolved in THF $\left(200 \mathrm{~cm}^{3}\right)$ and cooled to $0^{\circ} \mathrm{C}$. Butyl lithium $\left(1.6 \mathrm{M}\right.$ in hexane, $56.3 \mathrm{~cm}^{3}, 0.09 \mathrm{~mol}$ ) was added and the reaction mixture stirred at $0^{\circ} \mathrm{C}$ for $20 \mathrm{~min}$, then cooled to $-78^{\circ} \mathrm{C}$. Acetyl chloride $\left(7.3 \mathrm{~cm}^{3}\right.$, $0.1035 \mathrm{~mol}$ ) was added dropwise and the reaction mixture stirred at $-78^{\circ} \mathrm{C}$ for $\mathrm{ca} .4$ $\mathrm{h}$, then quenched with saturated aqueous ammonium chloride solution $\left(100 \mathrm{~cm}^{3}\right)$ and extracted with dichloromethane $\left(4 \times 100 \mathrm{~cm}^{3}\right)$. The combined organic extracts were dried, evaporated and purified by flash column chromatography $(1: 2$, ethyl acetatelight petroleum) to give the product $\left(R_{\mathrm{f}} 0.64,1: 1\right.$ ethyl acetate-light petroleum) as a pale yellow oil $(11.7 \mathrm{~g}, 76 \%)$.

$[\alpha]_{D}-70.36\left(c\right.$ 2.8, $\mathrm{CH}_{2} \mathrm{Cl}_{2}$ ) (Found: $171.0895 \mathrm{C}_{8} \mathrm{H}_{13} \mathrm{NO}_{3}$ requires 171.0895); $V_{\text {max. }}\left(\right.$ film) $2950 \mathrm{~m}, 1780 \mathrm{~s}, 1710 \mathrm{~s}, 1380 \mathrm{~s}, 1310 \mathrm{~s}, 1210 \mathrm{~s} \mathrm{~cm}^{-1} ; \delta_{\mathrm{H}}\left(250 \mathrm{MHz}, \mathrm{CDCl}_{3}\right)$ $0.89\left(3 \mathrm{H}, \mathrm{d}, J 6.9, \mathrm{CH}_{3} \mathrm{CHCH}_{3}\right), 0.92\left(3 \mathrm{H}, \mathrm{d}, J 7.1, \mathrm{CH}_{3} \mathrm{CHCH}_{3}\right), 2.38(1 \mathrm{H}, \mathrm{m}$, $\left.\mathrm{CH}_{3} \mathrm{CHCH}_{3}\right), 2.55\left(3 \mathrm{H}, \mathrm{s}, \mathrm{COCH}_{3}\right), 4.35(3 \mathrm{H}, \mathrm{m}, 5-\mathrm{HH}$ and $4-H) ; \delta_{\mathrm{C}}(62.9 \mathrm{MHz}$, $\left.\mathrm{CDCl}_{3}\right) \quad 14.63\left(\mathrm{CH}_{3} \mathrm{CHCH}_{3}\right), \quad 17.95\left(\mathrm{CH}_{3} \mathrm{CHCH}_{3}\right), 23.79\left(\mathrm{CH}_{3} \mathrm{CHCH}_{3}\right), \quad 28.35$ 
$\left(\mathrm{COCH}_{3}\right), 58.34(\mathrm{C}-4), 63.37(\mathrm{C}-5), 154.36(\mathrm{C}-2), 170.31\left(\mathrm{C}-\mathrm{l}^{\prime}\right) ; \mathrm{m} / \mathrm{z}$ (EI) $171\left(\mathrm{M}^{+}\right.$, $31.4 \%), 128(73.0), 86(100)$.

(4R)-4-Isopropyl-3-[3'- $\left.{ }^{2} \mathbb{H}_{3}\right]$ propionyloxazolidin-2-one 123<smiles>CC(C)[C@H]1COC(=O)N1C(=O)CC(C)(C)C</smiles>

Diisopropylamine $\left(11.6 \mathrm{~cm}^{3}, 0.083 \mathrm{~mol}\right)$ was added to THF $\left(80 \mathrm{~cm}^{3}\right)$ and the solution cooled to $0^{\circ} \mathrm{C}$. Butyl lithium( $1.6 \mathrm{M}$ in hexane, $\left.47.8 \mathrm{~cm}^{3}, 0.077 \mathrm{~mol}\right)$ was added dropwise and the reaction mixture stirred for $20 \mathrm{~min}$ at $0{ }^{\circ} \mathrm{C}$. The solution of LDA thus formed was cooled to $-78{ }^{\circ} \mathrm{C}$ and added dropwise to a stirred solution of the $\mathrm{N}$ acetyloxazolidinone $122(12.8 \mathrm{~g}, 0.075 \mathrm{~mol})$ in DMPU-THF $\left(1: 1 \mathrm{v} / \mathrm{v}, 160 \mathrm{~cm}^{3}\right)$ at $78{ }^{\circ} \mathrm{C}$ and the reaction stirred at that temperature for $30 \mathrm{~min} .\left[{ }^{2} \mathrm{H}_{3}\right]-$ Methyl iodide $\left(10.9 \mathrm{~cm}^{3}, 0.175 \mathrm{~mol}\right)$ was added dropwise to the stirred mixture and the stirring continued at $-78^{\circ} \mathrm{C}$ for $30 \mathrm{~min}$ before allowing the mixture to warm slowly to $0^{\circ} \mathrm{C}$ and then stirring for a further $4 \mathrm{~h}$. After this time, the reaction was quenched with hydrochloric acid $\left(3 \mathrm{M}, 80 \mathrm{~cm}^{3}\right)$ and the mixture extracted with diethyl ether $(4 \times 100$ $\mathrm{cm}^{3}$ ). The combined ether layers were then washed with saturated aqueous sodium chloride solution $\left(3 \times 100 \mathrm{~cm}^{3}\right)$ (to remove the DMPU), dried, evaporated and 
purified by flash column chromatography (1:4, ethyl acetate-light petroleum) to give the title compound $\left(R_{\mathrm{f}} 0.74,1: 1\right.$ ethyl acetate-light petroleum) $(5.42 \mathrm{~g}, 45 \%)$.

mp 134-135 ${ }^{\circ} \mathrm{C} ;[\alpha]_{\mathrm{D}}-91.3\left(c 2, \mathrm{CH}_{2} \mathrm{Cl}_{2}\right)$ (Found: 188.1240. $\mathrm{C}_{9} \mathrm{H}_{12} \mathrm{D}_{3} \mathrm{NO}_{3}$ requires $188.1240) ; v_{\max }(\mathrm{film}) 2950 \mathrm{~m}, 1780 \mathrm{~s}, 1720 \mathrm{~s}, 1390 \mathrm{~s}, 1250 \mathrm{~s}, 1210 \mathrm{~s} \mathrm{~cm}^{-1} ; \delta_{\mathrm{H}}(250 \mathrm{MHz}$, $\left.\mathrm{CDCl}_{3}\right) 0.86\left(3 \mathrm{H}, \mathrm{d}, J 6.9, \mathrm{CH}_{3} \mathrm{CHCH}_{3}\right), 0.92\left(3 \mathrm{H}, \mathrm{d}, J 7.1, \mathrm{CH}_{3} \mathrm{CHCH}_{3}\right), 2.36(1 \mathrm{H}$, m, $\left.\mathrm{CH}_{3} \mathrm{CHCH}_{3}\right), 2.92\left(2 \mathrm{H}, \mathrm{m}, \mathrm{COCH}_{2} \mathrm{CD}_{3}\right), 4.35(3 \mathrm{H}, \mathrm{m}, 5-\mathrm{HH}$ and $4-H) ; \delta_{\mathrm{C}}(62.9$ $\left.\mathrm{MHz}, \mathrm{CDCl}_{3}\right) 15.0\left(\mathrm{CH}_{3} \mathrm{CHCH}_{3}\right), 18.3\left(\mathrm{CH}_{3} \mathrm{CHCH}_{3}\right), 28.7\left(\mathrm{CH}_{3} \mathrm{CHCH}_{3}\right), 29.3$ $\left(\mathrm{COCH}_{2} \mathrm{CD}_{3}\right), 58.8(\mathrm{C}-4), 63.8(\mathrm{C}-5), 154.5(\mathrm{C}-2), 174.5\left(\mathrm{C}-1^{\prime}\right) ; \mathrm{m} / z(\mathrm{EI}) 188\left(\mathrm{M}^{+}\right.$, $18.0 \%), 145(26.4), 60(100)$.

$\left(2^{\prime} S, 4 R\right)-4-\left[\right.$ sopropyl-3- $\left(2^{\prime}-\left[{ }^{2} \mathbb{H}_{3}\right]\right.$ methyl- $3^{\prime}$-oxobutanoyl)oxazolidin-2-one $124^{104}$<smiles>CC(=O)[C@H](C)C(=O)N1C(=O)OC[C@H]1C(C)C</smiles>

To a stirred solution of diisopropylamine $\left(2.3 \mathrm{~cm}^{3}, 16 \mathrm{mmol}\right)$ in THF $\left(40 \mathrm{~cm}^{3}\right)$ at 0 ${ }^{\circ} \mathrm{C}$, butyl lithium $\left(1.6 \mathrm{M}\right.$ in hexane, $\left.10.1 \mathrm{~cm}^{3}, 16 \mathrm{mmol}\right)$ was added and the mixture stirred for $20 \mathrm{~min}$ at $0^{\circ} \mathrm{C}$. The LDA thus formed was then added to a solution of the $N$-propionyloxazolidinone $123(3.0 \mathrm{~g}, 16 \mathrm{mmol})$ in $\mathrm{THF}\left(60 \mathrm{~cm}^{3}\right)$ at $0{ }^{\circ} \mathrm{C}$ under nitrogen and the reaction was cooled to $-78^{\circ} \mathrm{C}$ and stirred at that temperature for 1.5

h. Acetyl chloride $\left(1.4 \mathrm{~cm}^{3}, 17.8 \mathrm{mmol}\right)$ in $\operatorname{THF}\left(10 \mathrm{~cm}^{3}\right)$ at $0{ }^{\circ} \mathrm{C}$ was then added to 
the reaction mixture (over $<5 \mathrm{~min}$ ) and it was stirred at $-78^{\circ} \mathrm{C}$ for $30 \mathrm{~min}$ before being quenched with saturated aqueous sodium chloride $\left(25 \mathrm{~cm}^{3}\right)$ and diluted with $\mathrm{H}_{2} \mathrm{O}$ to dissolve solids. The mixture was extracted with dichloromethane $(4 \times 60$ $\left.\mathrm{cm}^{3}\right)$, washed with saturated sodium hydrogen carbonate $\left(2 \times 50 \mathrm{~cm}^{3}\right)$ and brine $(50$ $\mathrm{cm}^{3}$ ), dried, evaporated and purified by recrystallisation (ethyl acetate-light petroleum) or flash column chromatography (1:4, ethyl acetate-light petroleum) to give the title compound $\left(R_{\mathrm{f}} 0.60,1: 1\right.$ ethyl acetate-light petroleum) as white crystals $(1.66 \mathrm{~g}, 47 \%)$.

mp. $134-135{ }^{\circ} \mathrm{C} ;[\alpha]_{\mathrm{D}}+20.2\left(c 1, \mathrm{CH}_{2} \mathrm{Cl}_{2}\right)$ (Found 230.13467. $\mathrm{C}_{11} \mathrm{H}_{14} \mathrm{D}_{3} \mathrm{NO}_{4}$ requires 230.1346); $v_{\text {max. }}$ (nujol) $2900 \mathrm{~m}, 1790 \mathrm{~s}, 1780 \mathrm{~s}, 1720 \mathrm{~s}, 1400 \mathrm{~s}, 1390 \mathrm{~s} \mathrm{~cm}^{-1}$; $\delta_{\mathrm{H}}\left(300 \mathrm{MHz}, \mathrm{CDCl}_{3}\right) 0.92\left(3 \mathrm{H}, \mathrm{d}, J 6.9, \mathrm{CH}_{3} \mathrm{CHCH}_{3}\right), 0.93(3 \mathrm{H}, \mathrm{d}, J$ 7.1, $\left.\mathrm{CH}_{3} \mathrm{CHCH}_{3}\right), 2.32\left(3 \mathrm{H}, \mathrm{s}, \mathrm{COCH}_{3}\right), 2.49\left(1 \mathrm{H}, \mathrm{m}, \mathrm{CH}_{3} \mathrm{CHCH}_{3}\right), 4.35(2 \mathrm{H}, \mathrm{m}, 5-\mathrm{HH}$ and $4-H), 4.49\left[\begin{array}{llll}1 & \mathrm{H} & \text { br } & \mathrm{s}, \mathrm{COCH}\left(\mathrm{CD}_{3}\right) \mathrm{CO}\end{array} ; \delta_{\mathrm{C}}\left(62.9 \mathrm{MHz}, \mathrm{CDCl}_{3}\right) 14.8\right.$ $\left(\mathrm{CH}_{3} \mathrm{CHCH}_{3}\right), \quad 18.3\left(\mathrm{CH}_{3} \mathrm{CHCH}_{3}\right), \quad 28.7\left(\mathrm{CH}_{3} \mathrm{CHCH}_{3}\right), 28.8\left(\mathrm{COCH}_{3}\right), \quad 53.3$ $\left[\mathrm{COCH}\left(\mathrm{CD}_{3}\right)\right], 59.0(\mathrm{C}-4), 64.0(\mathrm{C}-5), 154.7(\mathrm{C}-2), 170.1\left(\mathrm{C}-1^{\prime}\right), 205.9\left(\mathrm{C}-3^{\prime}\right) ; \mathrm{m} / \mathrm{z}$ (EI) $230\left(\mathrm{M}^{+}, 1.1 \%\right), 215(2.5), 188(100), 131(26.7), 102(39.8), 85(36.5)$.

$\left(2^{\prime} S, 3^{\prime} S, 4 R\right)-4 \cdot$ Isopropyl-3- $\left(2^{\prime} \cdot\left[{ }^{2} \mathbb{H}_{3}\right]\right.$ methyl- $3^{\prime}-\left[{ }^{2} \mathbb{H}_{1}\right]$ hydroxybutanoyl $)$ oxazolidin-2-one 125 and $\left(2^{\prime} S, 3^{\prime} \mathbb{R}, 4 \mathbb{R}\right)-4$-isopropyl-3- $\left(2^{\prime}-\left[{ }^{2} \mathbb{H}_{3}\right]\right.$ methyl-3' $\left[{ }^{2} \mathrm{H}_{1}\right]$ hydroxybutanoyl)oxazolidim-2-one 126 


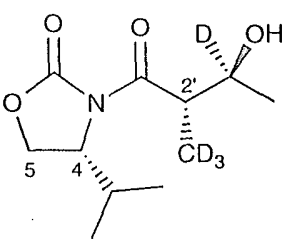

125

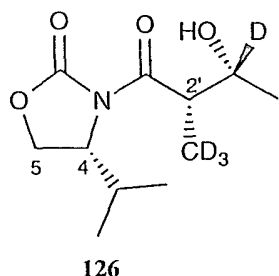

126

Compound $124(1.5 \mathrm{~g}, 6.6 \mathrm{mmol})$ was dissolved in a methanol-dichloromethane solvent mixture $\left(1: 1 \mathrm{v} / \mathrm{v}, 75 \mathrm{~cm}^{3}\right)$. To this solution sodium borodeuteride $(0.51 \mathrm{~g}$, $13.2 \mathrm{mmol})$ in methanol-dichloromethane $\left(1: 1 \mathrm{v} / \mathrm{v}, 30 \mathrm{~cm}^{3}\right)$ was added and the reaction stirred at $0{ }^{\circ} \mathrm{C}$ for $30 \mathrm{~min}$. The reaction mixture was quenched with hydrochloric acid $\left(5 \% \mathrm{v} / \mathrm{v}, 50 \mathrm{~cm}^{3}\right)$ and extracted with diethyl ether $\left(4 \times 100 \mathrm{~cm}^{3}\right)$. The combined ether extracts were washed with saturated aqueous ammonium chloride $\left(2 \times 50 \mathrm{~cm}^{3}\right)$ and sodium chloride $\left(50 \mathrm{~cm}^{3}\right)$, dried, evaporated and purified by flash column chromatography (1:3.5, ethyl acetate-light petroleum) to give the product $\left(R_{\mathrm{f}} 0.54,1: 1\right.$ ethyl acetate-light petroleum) as a mixture (1:2) of diastereoisomers $(0.8 \mathrm{~g}, 50 \%)$ although assignment of stereochemistry to the major diastereoisomer was not possible at this stage.

$V_{\text {max. }}\left(\right.$ film) $3500 \mathrm{~s}, 2900 \mathrm{~s}, 1780 \mathrm{~s}, 1690 \mathrm{~s}, 1460 \mathrm{w}, 1390 \mathrm{~s} \mathrm{~cm}^{-1} ; \delta_{\mathrm{H}}\left(250 \mathrm{MHz}, \mathrm{CDCl}_{3}\right)$ $0.9\left(3 \mathrm{H}, \mathrm{d}, J 7.3, \mathrm{CH}_{3} \mathrm{CHCH}_{3}\right), 0.94\left(3 \mathrm{H}, \mathrm{d}, J 7.4, \mathrm{CH}_{3} \mathrm{CHCH}_{3}\right), 1.21$ [major diastereoisomer: $3 \mathrm{H}, \mathrm{s}, \mathrm{CD}(\mathrm{OH}) \mathrm{CH}_{3}$ ], 1.28 [minor diastereoisomer: $3 \mathrm{H}, \mathrm{s}$, $\left.\mathrm{CD}(\mathrm{OH}) \mathrm{CH}_{3}\right], 2.39\left(1 \mathrm{H}, \mathrm{m}, \mathrm{CH}_{3} \mathrm{CHCH}_{3}\right.$ ), 2.53 (1 H, br s, OH), 3.84 (major diastereoisomer: $1 \mathrm{H}$, br s, $\mathrm{COCHCD}$ ), 3.89 (minor diastereoisomer: $1 \mathrm{H}$, br s, $\mathrm{COCHCD} 3), 4.34(3 \mathrm{H}, \mathrm{m}, \quad 5-\mathrm{HH}$ and $4-H) ; \delta_{\mathrm{C}}\left(62.9 \mathrm{MHz}, \mathrm{CDCl}_{3}\right) 15.1$ $\left(\mathrm{CH}_{3} \mathrm{CHCH}_{3}\right), 18.4\left(\mathrm{CH}_{3} \mathrm{CHCH}_{3}\right), 19.7\left[\mathrm{CD}(\mathrm{OH}) \mathrm{CH}_{3}\right], 28.9\left(\mathrm{CH}_{3} \mathrm{CHCH}_{3}\right), 43.0$ 
$\left(\mathrm{COCHCD}_{3}\right), 59.0(\mathrm{C}-4), 63.8(\mathrm{C}-5), 154.7(\mathrm{C}-2), 176.9\left(\mathrm{C}-\mathrm{I}^{\prime}\right) ; \mathrm{m} / \mathrm{z}$ ( $\left.\mathrm{EI}\right) 233\left(\mathrm{M}^{+}\right.$, $2.0 \%), 188(100), 130(45.9), 86(55.4)$.

$\left(2^{\prime} S, 3^{\prime} S, 4 R\right)-3-\left\{3^{\prime}-\left(3^{\prime \prime}, 5^{\prime \prime}-\right.\right.$ Dinitrobenzoyloxy $)-2^{\prime}-\left[{ }^{2} \mathrm{H}_{3}\right]$ methyl[ $\left.3^{\prime}-{ }^{2} \mathrm{H}_{1}\right]$ butanoyl $\}$-4-isopropyloxazolidin-2-one 127 and $\left(2^{\prime} S, 3^{\prime} R, 4 R\right)-3-\left\{3^{\prime}-\left[{ }^{2} H_{1}\right]-\left(3^{\prime \prime}, 5^{\prime \prime}-\right.\right.$ dinitrobenzoyloxy)-2'-[ $\left.{ }^{2} \mathrm{H}_{3}\right]$ methyl[ $\left[3^{\prime} \cdot{ }^{2} \mathrm{H}_{1}\right]$ butanoyl $\}-4$-isopropyloxazolidin-2-one 128<smiles>[2H][C@@](C)([CH]C(=O)N1C(=O)OC[C@H]1C(C)C)OC(=O)c1cc([N+](=O)[O-])cc([N+](=O)[O-])c1</smiles><smiles>[2H][C@](C)(OC(=O)c1cc([N+](=O)[O-])cc([N+](=O)[O-])c1)[C@@H](C)C(=O)N1C(=O)OC[C@@H]1C(C)C</smiles>

The mixture of alcohols 125 and $\mathbb{1 2 6}(0.83 \mathrm{~g}, 3.7 \mathrm{mmol})$ was dissolved in dichloromethane $\left(50 \mathrm{~cm}^{3}\right)$. 3,5-Dinitrobenzoyl chloride $(1.7 \mathrm{~g}, 7.4 \mathrm{mmol})$ was added and the solution turned yellow colour. Pyridine $\left(0.65 \mathrm{~cm}^{3}, 8.14 \mathrm{mmol}\right)$ was added slowly and the reaction was warmed to $c a .30^{\circ} \mathrm{C}$ and stirred for $2 \mathrm{~h}$ (solution went cream with a precipitate). The reaction was quenched with dilute hydrochloric acid and extracted with dichloromethane $\left(3 \times 50 \mathrm{~cm}^{3}\right)$. The combined dichloromethane 
extracts were then washed with dilute hydrochloric acid $\left(3 \times 50 \mathrm{~cm}^{3}\right)$, washed with saturated aqueous sodium chloride, dried and evaporated to give a mixture of diastereoisomers $(1.0 \mathrm{~g})$. Column chromatography was used to separate the diastereoisomers (1:99, ethyl acetate-toluene), to give $82 \mathrm{mg}$ of $127\left(R_{\mathrm{f}} 0.54,1: 3\right.$ ethyl acetate-toluene)and $500 \mathrm{mg}$ of $\mathbb{1 2 8}\left(R_{\mathrm{f}} 0.63,1: 3\right.$ ethyl acetate-toluene). The remainder was stored as a mixture (100 $\mathrm{mg}$

127 Desired isomer $(2 ' S, 3 ' S)$

(Found: 427.1529. $\mathrm{C}_{18} \mathrm{H}_{17} \mathrm{D}_{4} \mathrm{~N}_{3} \mathrm{O}_{9}$ requires 427.1530); $v_{\text {max. }}$ (film) $2900 \mathrm{~m}, 1780 \mathrm{~s}$, $1740 \mathrm{~m}, 1700 \mathrm{~m}, 1550 \mathrm{~s}, 1380 \mathrm{~m}, 1350 \mathrm{~s} \mathrm{~cm}^{-1} ; \delta_{\mathrm{H}}\left(300 \mathrm{MHz}, \mathrm{CDCl}_{3}\right) 0.75(3 \mathrm{H}, \mathrm{d}, J 6.9$, $\left.\mathrm{CH}_{3} \mathrm{CHCH}_{3}\right), 0.81\left(3 \mathrm{H}, \mathrm{d}, J\right.$ 7.0, $\left.\mathrm{CH}_{3} \mathrm{CHCH}_{3}\right), 1.47\left(3 \mathrm{H}, \mathrm{s}, \mathrm{CDOCH}_{3}\right), 2.17(1 \mathrm{H}$, $\left.\mathrm{m}, \mathrm{CH}_{3} \mathrm{CHCH}_{3}\right), 4.36\left(4 \mathrm{H}, \mathrm{m}, 5-\mathrm{HH}\right.$ and $\mathrm{COCHCD}_{3}$ and $\left.4-H\right), 9.19(3 \mathrm{H}, \mathrm{m}$, $\left.\mathrm{C}_{6} \mathrm{H}_{3} \mathrm{~N}_{2} \mathrm{O}_{4}\right) ; \delta_{\mathrm{C}}\left(62.9 \mathrm{MHz}, \mathrm{CDCl}_{3}\right) 14.9\left(\mathrm{CH}_{3} \mathrm{CHCH}_{3}\right), 18.0\left(\mathrm{CH}_{3} \mathrm{CHCH}_{3}\right), 18.4$ $\left(\mathrm{CDOCH}_{3}\right), 28.7\left(\mathrm{CH}_{3} \mathrm{CHCH}_{3}\right), 42.0\left(\mathrm{COCHCD}_{3}\right), 59.0(\mathrm{C}-4), 63.8(\mathrm{C}-5), 122.8$, 128.6, 129.4, 129.8, 134.5 (Ph C-1), $149.1\left(\mathrm{CODCH}_{3}\right), 154.4(\mathrm{C}-2), 162.2(\mathrm{CO}-\mathrm{Ph})$; $m / z(\mathrm{EI}) 427\left(\mathrm{M}^{+}, 1.3 \%\right), 382(4.5), 299$ (47.3), 215 (100), 195 (91.0), $149(43.4), 87$ (88.6).

128 Controll isomer $\left(2 ' S, 3^{\prime} R\right)$ 
(Found: 427.1529. $\mathrm{C}_{18} \mathrm{H}_{17} \mathrm{D}_{4} \mathrm{~N}_{3} \mathrm{O}_{9}$ requires 427.1530); $v_{\text {max. }}$ (nujol) $2900 \mathrm{~m}, 1780 \mathrm{~s}$, $1740 \mathrm{~m}, 1700 \mathrm{~m}, 1550 \mathrm{~s}, 1380 \mathrm{~m}, 1350 \mathrm{sm}^{-1} ; \delta_{\mathrm{H}}\left(300 \mathrm{MHz}, \mathrm{CDCl}_{3}\right) 0.91(3 \mathrm{H}, \mathrm{d}, J 7.1$, $\left.\mathrm{CH}_{3} \mathrm{CHCH}_{3}\right), 0.93\left(3 \mathrm{H}, \mathrm{d}, J 6.9, \mathrm{CH}_{3} \mathrm{CHCH}_{3}\right), 1.49\left(3 \mathrm{H}, \mathrm{s}, \mathrm{CDOCH}_{3}\right), 2.33(1 \mathrm{H}$, $\left.\mathrm{m}, \mathrm{CH}_{3} \mathrm{CHCH}_{3}\right), 4.14(1 \mathrm{H}$, br s, $\mathrm{COCHCD}$ ), $4.37(3 \mathrm{H}, \mathrm{m}, 5-\mathrm{HH}$ and 4-H), $9.19(3$ $\left.\mathrm{H}, \mathrm{m}, \mathrm{C}_{6} \mathrm{H}_{3} \mathrm{~N}_{2} \mathrm{O}_{4}\right) ; \delta_{\mathrm{C}}\left(62.9 \mathrm{MHz}, \mathrm{CDCl}_{3}\right) 14.9\left(\mathrm{CH}_{3} \mathrm{CHCH}_{3}\right), 18.0\left(\mathrm{CH}_{3} \mathrm{CHCH}_{3}\right)$, $18.4\left(\mathrm{CDOCH}_{3}\right), 28.7\left(\mathrm{CH}_{3} \mathrm{CHCH}_{3}\right), 42.0\left(\mathrm{COCHCD}_{3}\right), 59.0(\mathrm{C}-4), 63.8(\mathrm{C}-5)$, $122.8,125.3,128.6,129.4,129.8,134.5(\mathrm{Ph}-\mathrm{C}), 149.1\left(\mathrm{CDOCH}_{3}\right), 154.4(\mathrm{C}-2)$, 162.2 (CO-Ph); m/z (EI) $427\left(\mathrm{M}^{+}, 1 \%\right), 382(2.3), 299$ (48.1), 215 (90.8), 195 (95.1), $149(45.8), 87(100)$.

$(2 S, 3 R)-3-H y d r o x y-2-\left[{ }^{2} \mathbb{H}_{3}\right]$ methyl[ $\left[3-{ }^{2} \mathbb{H}\right]$ butanoic acid 129 (not isolated) ${ }^{1.38}$<smiles>[2H]C([2H])(O)[C@H](C(=O)O)C(C)(C)O</smiles>

To a solution of the 3,5-dinitrobenzoate $\mathbf{1 2 7}(85 \mathrm{mg}, 0.21 \mathrm{mmol})$ in THF-water (4:1, $\left.20 \mathrm{~cm}^{3}\right)$ under nitrogen, hydrogen peroxide $\left(30 \% \mathrm{w} / \mathrm{v}, 0.19 \mathrm{~cm}^{3}, 1.68 \mathrm{mmol}\right)$, and lithium hydroxide $(36 \mathrm{mg}, 0.84 \mathrm{mmol})$ in water $\left(5 \mathrm{~cm}^{3}\right)$, were added. The reaction was then stirred at $0^{\circ} \mathrm{C}$ for $6 \mathrm{~h}$ then quenched with sodium sulfite $(212 \mathrm{mg}, 1.68$ mmol) in water $\left(7 \mathrm{~cm}^{3}\right)$. The THF was removed in vacuo and the resulting mixture was extracted with dichloromethane $\left(4 \times 20 \mathrm{~cm}^{3}\right)$ to remove the chiral auxiliary 121 which was isolated in $74 \%$ yield. The aqueous layer was acidified to $\mathrm{pH} 1$ with dilute hydrochloric acid and saturated aqueous sodium chloride solution was added 
(to assist in the extraction of acids). The acids were then extracted with ethyl acetate $\left(5 \times 20 \mathrm{~cm}^{3}\right)$ and the combined organic extracts dried and evaporated to give a mixture of the title compound $\mathbb{1 2 9}$ and dinitrobenzoic acid 130 , of combined weight $44 \mathrm{mg}(63 \%)$.

$\delta_{\mathrm{H}}\left(90 \mathrm{MHz}, \mathrm{CDCl}_{3}\right) 129: 1.26\left[3 \mathrm{H}, \mathrm{s}, \mathrm{CD}(\mathrm{OH}) \mathrm{CH}_{3}\right], 2.61\left[1 \mathrm{H}\right.$, br s, $\left.\mathrm{COCH}\left(\mathrm{CD}_{3}\right)\right]$, $7.81(1 \mathrm{H}$, br s, $\mathrm{OH}) ; 130: 7.81(1 \mathrm{H}$, br s, $\mathrm{OH}), 9.20\left(3 \mathrm{H}, \mathrm{m}, \mathrm{C}_{6} \mathrm{H}_{3} \mathrm{~N}_{2} \mathrm{O}_{4}\right)$.

(2S,3S)-3-Hydroxy-2-[ $\left[{ }^{2} \mathrm{H}_{3}\right]$ methyl[ $\left[3{ }^{2} \mathrm{H}_{1}\right]$ butanoic acid 131 (not isolated) ${ }^{138}$<smiles>CC(O)(O)C(C(=O)O)C(=O)O</smiles>

The procedure was carried out as described above using the following; 3.5dinitrobenzoate $128(744 \mathrm{mg}, 1.83 \mathrm{mmol})$ in $\mathrm{THF}-\mathrm{H}_{2} \mathrm{O}\left(4: 1,150 \mathrm{~cm}^{3}\right)$, hydrogen peroxide (30\% w/v, $\left.1.64 \mathrm{~cm}^{3}, 14.64 \mathrm{mmol}\right)$, lithium hydroxide (310 $\left.\mathrm{mg}, 7.32 \mathrm{mmol}\right)$ in $\mathrm{H}_{2} \mathrm{O}\left(40 \mathrm{~cm}^{3}\right)$, sodium sulfite $(1.84 \mathrm{~g}, 14.64 \mathrm{mmol})$ in $\mathrm{H}_{2} \mathrm{O}\left(50 \mathrm{~cm}^{3}\right)$; to give 400 mg of the crude mixture of acids (67\%) and $178 \mathrm{mg}$ of the recovered chiral auxiliary, $75 \%)$.

$\delta_{\mathrm{H}}\left(90 \mathrm{MHz}, \mathrm{CDCl}_{3}\right) 131: 1.15\left[3 \mathrm{H}, \mathrm{s}, \mathrm{CD}(\mathrm{OH}) \mathrm{CH}_{3}\right], 2.30\left[1 \mathrm{H}\right.$, br s, $\left.\mathrm{COCH}\left(\mathrm{CD}_{3}\right)\right]$, $7.81\left(1 \mathrm{H}\right.$, br s, OH); 132: $7.81(\mathrm{l} \mathrm{H}, \mathrm{br} \mathrm{s}, \mathrm{OH}), 9.20\left(3 \mathrm{H}, \mathrm{m}, \mathrm{C}_{6} \mathrm{H}_{3} \mathrm{~N}_{2} \mathrm{O}_{4}\right)$. 
(2S,3S)-3-HHydroxy $2\left[^{2} \mathbb{H}_{3}\right]$ methyl[ $\left[3{ }^{2} \mathbb{H}_{1}\right]$ butanoyl $N$-acetylcysteamine 51 $\left\{S-2\right.$-(acetylamino)ethyl $(2 S, 3 S)-3-$ hyydroxy-2-[ $\left.{ }^{2} \mathrm{H}_{3}\right]$ methyl[3- $\left.{ }^{2} \mathrm{H}\right] \mathrm{butane}$ thioate\}<smiles>[2H]C([2H])(O)[C@H](C(=O)SCCNC(C)=O)[C@]([2H])(C)O</smiles>

DMAP (3.3 mg, $0.027 \mathrm{mmol})$ and $N$-acetylcysteamine $(64 \mathrm{mg}, 0.54 \mathrm{mmol})$ were added to a solution of the acids 129 and 130 (44 $\mathrm{mg}, 0.27 \mathrm{mmol}$ ) in diethyl etherdichloromethane $\left(1: 1 \mathrm{v} / \mathrm{v}, 15 \mathrm{~cm}^{3}\right)$ at $0^{\circ} \mathrm{C}$. DCC $(85 \mathrm{mg}, 0.41 \mathrm{mmol})$ was added and the solution was stirred for $10 \mathrm{~min}$ at $0{ }^{\circ} \mathrm{C}$ and then for a further $18 \mathrm{~h}$ at room temperature. The solution was then filtered through Celite and the Celite washed with ethyl acetate. The filtrate was evaporated to give an oil containing residual dicyclohexyurea (TLC system: acetone-toluene, $1: 1 ; R_{\mathrm{f}} 0.72$ ), the dinitrobenzoate thioester $\left(R_{\mathfrak{f}} 0.45\right)$, excess $N$-acetylcysteamine $\left(R_{\mathrm{f}} 0.35\right)$ and the product thioester $\left(R_{\mathrm{f}}\right.$ $0.2)$, which was isolated by flash chromatography $(1: 2$, acetone-toluene) to give the title compound as an oil ( $8 \mathrm{mg}, 30 \%)$.

$\nu_{\max }\left(\right.$ film) $3250 \mathrm{~s}, 2950 \mathrm{~s}, 1660 \mathrm{~s}, 1550 \mathrm{~s}, 1460 \mathrm{~m} \mathrm{~cm}^{-1} ; \delta_{\mathrm{H}}\left(250 \mathrm{MHz}, \mathrm{CDCl}_{3}\right) 1.24[3$ $\left.\mathrm{H}, \mathrm{s}, \mathrm{CD}(\mathrm{OH}) \mathrm{CH}_{3}\right], 1.97\left(3 \mathrm{H}, \mathrm{s}, \mathrm{CH}_{3} \mathrm{CONH}\right), 2.69\left[1 \mathrm{H}\right.$, br s, $\left.\mathrm{COCH}\left(\mathrm{CD}_{3}\right)\right], 3.05(2$ $\left.\mathrm{H}, \mathrm{m}, \mathrm{CH}_{2} \mathrm{~S}\right), 3.46\left(2 \mathrm{H}, \mathrm{m}, \mathrm{CH}_{2} \mathrm{NH}\right) ; \delta_{\mathrm{C}}\left(62.9 \mathrm{MHz}, \mathrm{CDCl}_{3}\right) 15.25\left(\mathrm{COCHCD}_{3}\right)$, $20.46\left(\mathrm{CH}_{3} \mathrm{CONH}\right), 23.61\left[\mathrm{CD}(\mathrm{OH}) \mathrm{CH}_{3}\right], 28.97\left(\mathrm{CH}_{2} \mathrm{~S}\right), 39.78\left(\mathrm{CH}_{2} \mathrm{NH}\right), 54.62$ $\left[\mathrm{COCH}\left(\mathrm{CD}_{3}\right)\right], 70.17\left[\mathrm{CD}(\mathrm{OH}) \mathrm{CH}_{3}\right], 171.95\left(\mathrm{CH}_{3} \mathrm{CONH}\right) 203.55(\mathrm{SCO}) ; \mathrm{m} / z(\mathrm{EI})$ $223\left(\mathrm{M}^{+}, 3.9 \%\right), 205(1.8), 149(58.4), 119(59.6), 60(100)$ 
$(2 S, 3 \mathbb{R})-3 \cdot \mathbb{H}\left[y d r o x y-2-\left[{ }^{2} \mathbb{H}_{3}\right]\right.$ methyl[ $\left.3{ }^{2}{ }^{2} \mathbb{H}_{1}\right]$ butanoyl $N$-acetylcysteamine 133 $\left\{S-2\right.$-(acetylamino)ethyl $(2 S, 3 R)-3-$ hydroxy-2- $\left[{ }^{2} \mathbb{H}_{3}\right]$ methyl[ $\left[3-^{2} \mathbb{H}\right]$ butanethioate<smiles>CC(=O)NCCSC(=O)[C@H]([18O])C(C)(O)O</smiles>

The procedure was carried out as described above using the following; acids 131 and $132(400 \mathrm{mg}, 2.42 \mathrm{mmol})$ in diethyl ether-dichloromethane $\left(1: 1 \mathrm{v} / \mathrm{v}, 150 \mathrm{~cm}^{3}\right)$, DMAP (29 mg, $0.242 \mathrm{mmol}$ ), $N$-acetylcysteamine ( $573 \mathrm{mg}, 4.84 \mathrm{mmol}$ ), DCC (749 $\mathrm{mg}, 3.63 \mathrm{mmol}$ ); to give the crude material which contained residual dicyclohexyurea (TLC system: acetone-toluene, $\left.1: 1 ; R_{\mathrm{f}} 0.72\right)$, the dinitrobenzoate thioester $\left(R_{\mathrm{f}} 0.45\right)$, excess $N$-acetylcysteamine $\left(R_{\mathrm{f}} 0.35\right)$ and the product thioester $\left(R_{\mathrm{f}} 0.2\right)$, which was isolated by flash chromatography (1:2, acetone-toluene) to give the title compound as an oil (16 mg, $40 \%)$.

$v_{\max .(f i l m)} 3300 \mathrm{~s}, 2950 \mathrm{~s}, 1660 \mathrm{~s}, 1550 \mathrm{~s}, 1460 \mathrm{~m} \mathrm{~cm}^{-1} ; \delta_{\mathrm{H}}\left(250 \mathrm{MHz}, \mathrm{CDCl}_{3}\right) 1.16[3 \mathrm{H}$, s, $\left.\mathrm{CD}(\mathrm{OH}) \mathrm{CH}_{3}\right], 1.90\left(3 \mathrm{H}, \mathrm{s}, \mathrm{CH}_{3} \mathrm{CONH}\right), 2.61\left[1 \mathrm{H}, \mathrm{br} \mathrm{s}, \mathrm{COCH}\left(\mathrm{CD}_{3}\right)\right], 2.97(2 \mathrm{H}$, m, $\left.\mathrm{CH}_{2} \mathrm{~S}\right), 3.38\left(2 \mathrm{H}, \mathrm{m}, \mathrm{CH}_{2} \mathrm{NH}\right) ; \delta_{\mathrm{C}}\left(62.9 \mathrm{MHz}, \mathrm{CDCl}_{3}\right) 19.06\left(\mathrm{COCHCD}_{3}\right), 22.19$ $\left(\mathrm{CH}_{3} \mathrm{CONH}\right), \quad 23.91 \quad\left[\mathrm{CD}(\mathrm{OH}) \mathrm{CH}_{3}\right], \quad 27.56\left(\mathrm{CH}_{2} \mathrm{~S}\right), \quad 38.35 \quad\left(\mathrm{CH}_{2} \mathrm{NH}\right), \quad 53.24$ [COCH $\left.\left(\mathrm{CD}_{3}\right)\right], 70.10\left[\mathrm{CD}(\mathrm{OH}) \mathrm{CH}_{3}\right], 169.90\left(\mathrm{CH}_{3} \mathrm{CONH}\right), 204.95(\mathrm{SCO}) ; \mathrm{m} / \mathrm{z}(\mathrm{EI})$ $223\left(\mathrm{M}^{+}, 2.4 \%\right), 149(54.3), 119(100), 60(96.7)$. 


\subsection{Biologicall experimentall details}

\subsubsection{Composition of culture media}

\subsubsection{Agar medium 1: AGS}

Glycerol (12.5 g), sodium chloride (1 g), oxoid agar technical no.3 (15 g), $\mathrm{K}_{2} \mathrm{HPO}_{4}(1$ g), $\mathrm{MgSO}_{4}{ }^{\circ} 7 \mathrm{H}_{2} \mathrm{O}(0.5 \mathrm{~g}), \mathrm{Fe}_{2}\left(\mathrm{SO}_{4}\right)_{3} \cdot 6 \mathrm{H}_{2} \mathrm{O}(0.01 \mathrm{~g}), \mathrm{CuSO}_{4}{ }^{\circ} 5 \mathrm{H}_{2} \mathrm{O}(0.001 \mathrm{~g})$, $\mathrm{ZnSO}_{4}{ }^{\circ} 7 \mathrm{H}_{2} \mathrm{O}(0.001 \mathrm{~g}), \mathrm{MnSO}_{4}{ }^{\circ} \mathrm{H}_{2} \mathrm{O}(0.001 \mathrm{~g}), 1000 \mathrm{~cm}^{3}$ water; adjusted to $\mathrm{pH} 7.0$ with dilute sodium hydroxide solution.

\subsubsection{Agar medium 2: ATCC no. 5}

Oxoid yeast extract ( $1 \mathrm{~g})$, oxoid beef extract ( $1 \mathrm{~g})$, tryptose $(2 \mathrm{~g})$, oxoid agar technical no. $3(15 \mathrm{~g}), \mathrm{FeSO}_{4}{ }^{\circ} 7 \mathrm{H}_{2} \mathrm{O}(0.001 \mathrm{~g}), 1000 \mathrm{~cm}^{3}$ water; adjusted to $\mathrm{pH} 7.0$ with dilute sodium hydroxide solution.

\subsubsection{Primary seed: glucose-yeast-extract medium (GYE)}

Glucose (10 g), spray dried yeatex (10 g) (SmithKline Beecham), $1000 \mathrm{~cm}^{3}$ water; adjusted to $\mathrm{pH} 7.0$ with dilute sodium hydroxide solution.

\subsubsection{Production medium: $\mathbb{F C A}$}

Fructose (20 g), collofilm dextrin (60 g) (SmithKline Beecham), arkasoy (30 g) (SmithKline Beecham), calcium carbonate $(10 \mathrm{~g}), 1000 \mathrm{~cm}^{3}$ water; adjusted to $\mathrm{pH}$ 7.7 with dilute sodium hydroxide solution. 


\subsubsection{Production medium: complex}

Glucose (50 g), pharmamedia (30 g), (SmithKline Beecham), calcium carbonate (10 g), $1000 \mathrm{~cm}^{3}$ water; adjusted to $\mathrm{pH} 7.7$ with dilute sodium hydroxide solution.

\subsubsection{Production medium: defined}

Glucose (50 g), L-asparigine (1 g), $\mathrm{FeSO}_{4} \circ 7 \mathrm{H}_{2} \mathrm{O}(0.1 \mathrm{~g}), \mathrm{MnCl}_{2} \circ 4 \mathrm{H}_{2} \mathrm{O}(0.1 \mathrm{~g})$, calcium carbonate $(10 \mathrm{~g}), 1000 \mathrm{~cm}^{3}$ water; adjusted to $\mathrm{pH} 7.7$ with dilute sodium hydroxide solution.

\subsubsection{Growth of Streptomyces nodosus}

\subsubsection{Preparation of vegetative culture}

Onto a previously prepared agar slant (Agar media 1 or 2) spores of $S$. nodosus (strain ATCC 14899) $\left(0.1 \mathrm{~cm}^{3}\right)$, stored at $-80^{\circ} \mathrm{C}$, were added and dispersed with a sterilised loop and incubated at $30^{\circ} \mathrm{C}$. A vegetative culture was prepared by dislodging the spores from the slant by the addition of sterilised Tween $80(0.02 \%$ $\mathrm{w} / \mathrm{v}, 5 \mathrm{~cm}^{3}$ ) (Sigma). This suspension was then added to a glucose-yeast-extract medium (GYE) $\left(2 \mathrm{~cm}^{3}\right.$ per $500 \mathrm{~cm}^{3}$ of medium; $100 \mathrm{~cm}^{3}$ of GYE per $500 \mathrm{~cm}^{3}$ Erlenmeyer flask) and was placed in an incubator shaker for $48 \mathrm{~h}\left(28^{\circ} \mathrm{C}, 350 \mathrm{rpm}\right)$. After this time the broth was mixed in a 1:1 ratio with sterilised CRY preservative $(20 \%$ glycerol $\mathrm{w} / \mathrm{v}, 10 \%$ lactose $\mathrm{w} / \mathrm{v}, 70 \%$ water). The vegetative culture was then stored at $-40^{\circ} \mathrm{C}$ in $1.5 \mathrm{~cm}^{3}$ aliquots. 


\subsubsection{Primary seed}

In order to initiate the growth of the vegetative culture of $S$. nodosus, initially a flask containing GYE medium was inoculated to produce a primary seed. The GYE medium $\left(100 \mathrm{~cm}^{3}\right)$ was made up and placed in an Erlenmeyer flask $\left(500 \mathrm{~cm}^{3}\right)$ with a cotton wool plug, which was then sterilised in an autoclave for 20 min $(2 \mathrm{~atm} ., 120$ ${ }^{\circ} \mathrm{C}$ ). (This procedure was generally carried out in duplicate in order to ensure the production of a satisfactory primary seed.) When this had cooled, an aliquot (1.0 $\mathrm{cm}^{3}$ ) of $S$. nodosus (ATCC 14899) vegetative suspension (defrosted) was added and the broth was incubated $\left(28^{\circ} \mathrm{C}, 350 \mathrm{rpm}\right)$ for $72 \mathrm{~h}$, after which time the spores had developed into spheres of vegetative cells, and this suspension was used to inoculate the production media.

\subsubsection{Production media}

The production media (FCA, complex or defined) were made up as required and portions $\left(60 \mathrm{~cm}^{3}\right)$ were added to Erlenmeyer flasks $\left(500 \mathrm{~cm}^{3}\right)$ (various size flasks could be used for the growth of $S$. nodosus, with the corresponding volumes of whole broth; $12 \% \mathrm{v} / \mathrm{v})$ with cotton wool plugs, which were then sterilised for $20 \mathrm{~min}(2$ atm., $\left.120^{\circ} \mathrm{C}\right)$. An inoculum of the primary seed $(5 \% \mathrm{v} / \mathrm{v})$ was then added to the media and the broth was incubated $\left(28^{\circ} \mathrm{C}, 400 \mathrm{rpm}\right)$ for $3-8$ days. Concentrations of amphotericin A and B generally peaked after 4-5 days and this was found to be the optimum time for harvesting the metabolites. 


\subsubsection{Quantitative determination of amphotericin $\mathbb{A}$ and $\mathbb{B}$ concentration ${ }^{71}$}

To a sample of culture broth $\left(1 \mathrm{~cm}^{3}\right)$ (or any other solution of unknown amphotericin A and/or B concentration) DMSO $\left(9 \mathrm{~cm}^{3}\right)$ was added, the solution was sonicated for $20 \mathrm{~min}$ at room temperature and then centrifuged $\left(10000 \mathrm{rpm}, 10 \mathrm{~min}, 20^{\circ} \mathrm{C}\right)$. The clear yellow-orange solution was diluted with methanol $(1: 10)$, with the dilution adjusted accordingly in the presence of higher or lower antibiotic concentrations. The absorption of the appropriate methanol dilution was recorded in $1 \mathrm{~cm}^{3}$ quartz cuvettes in the wavelength range 200 to $450 \mathrm{~nm}$. Amphotericin B showed four specific UV absorption peaks at 346, 364, 382 and $405 \mathrm{~nm}$, with its concentration being calculated on the basis of the absorption at $\lambda 405 \mathrm{~nm}$, assuming $\varepsilon_{1 \mathrm{~cm}}{ }^{1 \%}$ of 1725 (10 $\mu \mathrm{g} \mathrm{cm}^{-3}$ methanol). The UV spectrum of amphotericin A showed four specific tetraene peaks at $280,292,305$ and $320 \mathrm{~nm}$ and its concentration was determined on the basis of the absorption at $\lambda 305 \mathrm{~nm}$, assuming $\varepsilon_{1 \mathrm{~cm}}{ }^{1 \%} 840\left(10 \mu \mathrm{g} \mathrm{cm}^{-3}\right.$ methanol). The following equations were used for calculating the concentration of a solution ( $\mu \mathrm{g}$ $\mathrm{cm}^{-3}$ ): concentration of amphotericin $\mathrm{B}=$ absorbance $\times$ dilution $\times 5.797$ concentration of amphotericin $\mathrm{A}=$ absorbance $\times$ dilution $\times 11.765$.

\subsubsection{Extraction and purification of metabolites}

There were two general procedures applied when extracting metabolites from a fermentation broth. The first, liquid ion exchange extraction, was used for large scale experiments, when the broth was grown in $500 \mathrm{~cm}^{3}$ shake flasks and afforded pure amphotericin B in mg quantities. The second involved small levels of whole 
broth $\left(15 \mathrm{~cm}^{3}\right.$ grown up in $100 \mathrm{~cm}^{3}$ flasks) which was subjected to methanol extraction, followed by the isolation of a variety of metabolites by semi-preparative HPLC in $\mu$ g quantities. A third extraction process was undertaken in the search for minor co-metabolites of amphotericin B which involved the growth of a large quantity of broth, followed by a hot butanol extraction.

\subsubsection{Liquid ion exchange extraction}

(a) Extraction method. Fermentation broths were grown for between 3-7 days in $500 \mathrm{~cm}^{3}$ shake flasks. The broth was harvested when required and the $\mathrm{pH}$ was adjusted to 10.5 with aqueous sodium hydroxide solution $(5 \mathrm{M})$. The broth was then mixed for $1 \mathrm{~h}$ under continuous agitation (stirring at room temp.) with one half volume of ethyl acetate containing Aliquat $336(7 \% \mathrm{w} / \mathrm{v})$ (SmithKline Beecham), with the $\mathrm{pH}$ being maintained at 10.5 with further additions of sodium hydroxide solution $(5 \mathrm{M})$. The phases were then separated by centrifugation $\left(10000 \mathrm{rpm}, 20 \mathrm{~min}, 20^{\circ} \mathrm{C}\right.$ ) and the aqueous phase was discarded. The organic phase was then UV assayed to determine the amphotericin B content and was left to stand at $5{ }^{\circ} \mathrm{C}$ (in the fridge) for 3-5 days whilst precipitation of amphotericin $\mathrm{B}$ occurred. When the concentration of amphotericin B had fallen to less than $20 \%$ of the original level, the crude product was recovered from the mother liquor.

(b) Product recovery. The bulk of the mother liquor was decanted off and then the yellow precipitate of amphotericin B was resuspended in the remaining mother liquor. The product was recovered by subjecting this solution to centrifugation 
$\left(10000,10 \mathrm{~min}, 20^{\circ} \mathrm{C}\right)$. The solid phase was recovered and resuspended in deionised water and was once more recovered by centrifugation. This was then repeated by washing the solid with acetone and then methanol, before being dried under high vacuum to give amphotericin B as a bright yellow solid with a $95 \%$ purity.

\subsubsection{Freeze dried whole broth methanol extraction}

(a) Extraction method. The fermentation broth was grown for 3-5 days in $100 \mathrm{~cm}^{3}$ shake flasks containing FCA media $\left(15 \mathrm{~cm}^{3}\right)$. The broth was harvested when required and was freeze dried $\left(1 \mathrm{~h},-40{ }^{\circ} \mathrm{C}, 0.92 \mathrm{mbar} 1 \mathrm{~atm}^{-1}\right)$ to give a pale yellow powder. This was then extracted with methanol $\left(20 \mathrm{~cm}^{3}, 30 \mathrm{~min}\right.$, room temp.) after which the methanol was centrifuged $\left(10000 \mathrm{rpm}, 10 \mathrm{~min}, 20^{\circ} \mathrm{C}\right)$ to separate the supernatant and the solids. The solid pellet was resuspended in methanol and this extraction procedure was repeated up to six times, until the methanol extract showed minimal levels of amphotericin after UV assay. The methanol extracts were then combined and concentrated in vacuo to give a yellow, oily solid, which was redissolved (sonication, $10 \mathrm{~min}$ ) in a small amount of methanol-DMSO (1:1). Often, all the solid did not dissolve in this solvent mixture, which was therefore centrifuged $\left(10000 \mathrm{rpm}, 10 \mathrm{~min}, 20^{\circ} \mathrm{C}\right)$, the solvent decanted and the remaining solid redissolved in a further methanol-DMSO (1:1) solvent mixture. 
(b) Product isolation and recovery. A semi-preparative HPLC technique was used to isolate the amphotericin from the methanol extracts of the freeze-dried whole broth. Generally, a sample of known concentration of standard amphotericin B $\left(10 \mu \mathrm{g} \mathrm{cm}^{-3}\right.$ ) (SmithKline Beecham) was injected onto the HPLC to determine where the amphotericin B would run in the mobile phase system. The mobile phase used consisted of 75:25 HPLC-grade methanol-sodium citrate buffer $(0.05$ M, pH 5.3 adjusted with citric acid), detecting at o $305 \mathrm{~nm}$ for the tetraenes and o $346 \mathrm{~nm}$ for the heptaenes. The solvent and buffer used in the mobile phase were thoroughly degassed with helium prior to use. Before any injections were made, the reverse-phase C18 column was washed with methanol $(100 \%, 10 \mathrm{~min})$, followed by equilibration with the mobile phase $(20 \mathrm{~min})$. Amphotericin $\mathrm{A}$ and $\mathrm{B}$ peaks were collected (after repeated injections onto the HPLC column), the samples concentrated in vacuo and then redissolved in water-methanol $(1: 1, c a .3$ $\mathrm{cm}^{3}$ ) (solution was slightly cloudy). These samples were then passed down a HP20 diaion adsorption column, in order to remove the citrate salts from the HPLC buffer system. Prior to the addition of the sample onto the HP20 column, methanol was run through $(10 \mathrm{~min})$ followed by water $(10 \mathrm{~min})$ to clean the column. The sample was then loaded onto the HP20 column, with detection at the appropriate wavelength on the attached UV monitor. The column was first eluted with deionised water $(1 \mathrm{~h})$, after which methanol was run through. The yellow colour of the amphotericin could be seen to elute down the column and the fractions containing the amphotericin (detected by the UV monitor) were pooled and concentrated in vacuo. The yellow residue was then redissolved in a 
minimum volume of methanol $\left(<1 \mathrm{~cm}^{3}\right)$ which was then analysed by electrospray mass spectrometry.

\subsubsection{HHot butanol extraction}

(a) Extraction method. The fermentation broth was grown for 4 days in $2 \mathrm{dm}^{3}$ shake flasks containing FCA media $\left(240 \mathrm{~cm}^{3}\right)$, after which time the broth was harvested and was extracted with butanol $\left(2 \times 500 \mathrm{~cm}^{3}, 1 \mathrm{~h}\right.$ at $\left.c a .50{ }^{\circ} \mathrm{C}\right)$. The butanol extract was then reduced to dryness in vacuo and the resulting yellow-brown residue was dissolved, by sonicating ( $20 \mathrm{~min}$ ) in a solution of dimethyl formamide (DMF, $10 \mathrm{~cm}^{3}$ ) plus citric acid (10 mg). This extraction process was different to any previously undertaken, as it involved the addition of a quantity of citric acid in order to enhance the solubility of any metabolites present by forming the citrate salt.

(b) Product isolation and recovery. This extraction was carried out in the search for minor co-metabolites of amphotericin and so, initially, a crudely purification was undertaken to remove the amphotericin B. Samples of the extract were injected (1 $\mathrm{cm}^{3}$ ) onto a semi-preparative HPLC reverse phase C18 column using the mobile phase described previously (73:27, methanol-citrate buffer) detecting at o 405 $\mathrm{nm}$, from which the amphotericin B peak was collected and discarded whilst the remaining solute that was collected was pooled and concentrated in vacuo, before being redissolved by sonicating in DMF ( $5 \mathrm{~cm}^{3}$ with $5 \mathrm{mg}$ citric acid).

In order to get an indication of the content of the remaining extract an analytical reverse phase HPLC trace with a diode array facility (at SmithKline Beecham, 
Brockham Park) trace was taken of an injected sample detecting at o $305 \mathrm{~nm}$ (to detect any tetraenes) and at o $405 \mathrm{~nm}$ (to detect any heptaenes). The conditions for these injections $(10 \mu \mathrm{l})$ utilised a different solvent system to ensure any peaks present would be sufficiently separated, so as to identify individual peaks. The solvent program involved a gradual increase in concentration of mobile phase A in mobile phase B (0-100\% over $30 \mathrm{~min})$, followed by $20 \mathrm{~min}$ isocratic in mobile phase B [mobile phase A: 40:60, methanol-acetonitrile (3:2) : $\mathrm{NH}_{4} \mathrm{OAc}$ (aq., $0.001 \mathrm{~mol} \mathrm{dm}^{-3}$ ); mobile phase B: 80:20, methanol-acetonitrile (3:2) : $\mathrm{NH}_{4} \mathrm{OAc}$ (aq., $\left.0.001 \mathrm{~mol} \mathrm{dm}^{-3}\right]$. Once the content of the extract was determined, samples of the extract were injected $\left(1 \mathrm{~cm}^{3}\right)$ onto a semi-preparative HPLC column using the same mobile phase system, to isolate individual peaks. Fractions containing certain peaks could then be combined, concentrated, de-salted (as described above) and analysed by NMR or mass spectrometry.

\subsubsection{Addition of labelled compounds to the fermentation broth}

\subsubsection{1 $\left[1,2{ }^{13} \mathbb{C}_{2}\right]$ Acetate}

Four Erlenmeyer flasks $\left(500 \mathrm{~cm}^{3}\right)$ each containing FCA media $\left(60 \mathrm{~cm}^{3}\right)$ were prepared as previously outlined. $\left[1,2-{ }^{13} \mathrm{C}_{2}\right]$ Acetate was divided between four flasks and pulsed-fed to the whole broth over three days. The aliquots of acetate ( $42 \mathrm{mg}$ in $200 \mu 1$ of sterilised water per flask per feeding) were administered at $24,40,44,48$, 64, 68 and $70 \mathrm{~h}$ after inoculation. After $75 \mathrm{~h}$, the broth was harvested and the amphotericin B isolated as described above in Section 6.3.4.1. 


\subsubsection{2 $\left[1-^{13} \mathrm{C}\right]$ and $\left[2 \alpha^{13} \mathbb{C}\right]$ acetate}

Three Erlenmeyer flasks $\left(500 \mathrm{~cm}^{3}\right)$ each containing FCA media $\left(60 \mathrm{~cm}^{3}\right)$ were prepared as previously outlined. Aliquots of each acetate $(1 \mathrm{~g})$ were added to these three flasks over $70 \mathrm{~h} \mathrm{(42} \mathrm{mg}$ of each acetate in $200 \mu \mathrm{l}$ of sterilised water was added to each flask at $18,22,26,42,46,50,66$ and $70 \mathrm{~h}$ after inoculation). A fourth flask was inoculated and grown as a control, without the addition of any compounds. After $75 \mathrm{~h}$, the broth was harvested and the amphotericin B isolated as described above in Section 6.3.4.1.

\subsubsection{3 $\left[1-^{13} \mathbb{C}\right]$ Acetate and diethyl $\left[2-^{13} \mathbb{C}\right]$ malonate}

Four Erlenmeyer flasks $\left(500 \mathrm{~cm}^{3}\right)$ each containing FCA media $\left(60 \mathrm{~cm}^{3}\right)$ were prepared as previously outlined. Aliquots of the labelled acetate and malonate $(0.5$ $\mathrm{mg}$ of each) were added to the four shake flasks over a period of $72 \mathrm{~h}(21 \mathrm{mg}$ of each in $100 \mu 1 \mathrm{H}_{2} \mathrm{O}$ per feeding per flask at $21,25,42,46,50$ and $66 \mathrm{~h}$ after inoculation). After $75 \mathrm{~h}$, the broth was harvested and the amphotericin B isolated as described above in Section 6.3.4.1

\subsubsection{Labelled diketides}

Four Erlenmeyer flasks $\left(100 \mathrm{~cm}^{3}\right)$ each containing FCA media $\left(15 \mathrm{~cm}^{3}\right)$ were prepared as previously outlined. To the first flask, the labelled diketide $(51,10 \mathrm{mg}$, $200 \mu 1$ ethanol) was added ( $28 \mathrm{~h}$ after inoculation), along with compounds 3-PA, 3- 
TDPA and DMCD. Under exactly the same conditions, the control epimeric labelled diketide (133), as well as the above additional compounds, were fed to the second flask. As control experiments, a flask of whole broth was grown that had only the three extra compounds added, along with a flask that did not have any added compounds. After $75 \mathrm{~h}$ the broth was harvested and the amphotericin A and B isolated as described above in Section 6.3.4.2. 


\section{Referemces}

1 R. B Herbert, The Biosynthesis of Secondary Metabolites, Chapman and Hall, London, 2nd edn., 1989; S. Omura, Macrolide Antibiotics Chemistry, Biology and Practice, Academic Press, New York, 1984.

2 J. Mann, Secondary Metabolism, Clarendon Press, Oxford, 2nd edn., 1987.

3 J. N. Collie, Proc. Chem. Soc., 1907, 91, 230.

4 J. N. Collie, J. Chem. Soc., 1907, 91, 1806.

$5 \quad$ R. Robinson, J. Roy. Soc. Arts, 1948, 96, 795.

6 A. J. Birch, Science, 1967, 156, 202.

$7 \quad$ F. Lynen and E. Reichert, Angew. Chem., 1951, 6347.

8 D. Rittenberg and K. Bloch, J. Biol. Chem., 1945, 160, 417.

9 S. Omura, A. Nakagawa, H. Takeshima, J. Miyazawa, C. Kitao, F. Piriou and G. Lukacs, Tetrahedron Lett., 1975, 4503.

10 D. E. Cane H. Hasler, P. B.Taylor and T.-C. Liang, Tetrahedron, 1983, 39, 3449.

11 D. O'Hagan, Chem. Brit., 1990, 246.

12 D. A. Brindley, S. Matsumura and K. Bloch, Nature, 1969, 224, 666.

13 J. J. Volpe and P. R. Vagelos, Ann. Rev. Biochem., 1973, 42, 21.

14 T. A. Holak, S. K. Kearsley, Y. Kim and J. H. Prestegard, Biochemistry, $1988,27,6135$. 

Biochem. Biophys. Acta., 1973, 326, 155. S. M. Trandor, Bacteriol. Rev., 1976, 40, 681. G. Roberts and P. F. Leadlay, FEBS Lett., 1983, 159, 13. J. W. Westley, J. F. Blount, R. H. Evans, Jr., A. Stempel and J. Berger, J. Antib., 1974, 27, 597. Am. Chem. Soc., 1981, 103, 5953.

24 D. E. Cane, W. D. Celmer and J. W. Westley, J. Am. Chem. Soc., 1983, $105,3594$.

O. R. H. Oikawa, A. Ichihara and S. Sakarumi, J. Chem Soc., Chem. Commun., 1990, 908.

J. W. Westley, D. L. Pruess, R. G. Pitcher, J. Chem. Soc., Chem. Commun, $1972,161$.

C. Vederas, J. Am. Chem. Soc., 1988, 110, 2938.

30 S. Yue, J. S. Duncan, Y. Yamamoto and C. R. Hutchinson, J. Am. Chem. Soc., 1987, 109, 1253.

D. E. Cane and C.-C. Yang, J. Am. Chem. Soc., 1987, 109, 1255. 
Z. Li, F. M. Martin and J. C. Vederas, J. Am. Chem. Soc., 1992, 114, 1531 Y. Yoshizawa, Z. Lee, P. B. Reese and J. C. Vederas, J. Am. Chem. Soc., $1990,112,3212$.

D. E. Cane, R. H. Lambalot, P. C. Prabhakaran and W. R. Ott, J. Am. Chem. Soc., 1993, 115, 522.

H. Patzelt and J. A. Robinson, J. Chem. Soc., Chem. Commun., 1993, 1258.

M. J. Garson and J. Staunton, Chem. Soc. Rev., 1979, 539.

J. C. Vederas, J. Am. Chem. Soc., 1980, 102, 374.

J. Staunton and A. C. Sutkowski, J. Chem. Soc., Chem. Commun., 1991, 1113.

A. M. Hill, A. Jacobs and J. Staunton, J. Chem. Soc., Chem. Commun., 1995,859

A. M. Hill and J. Staunton, J. Chem. Soc., Chem. Commun., 1995, 861.

A. Jacobs and J. Staunton, J. Chem. Soc., Chem. Commun., 1995, 863.

H. C. Hailes, C. M. Jackson, P. F. Leadlay, S. V. Ley and J. Staunton, Tetrahedron Lett., 1994, 35, 307.

H. C. Hailes, S. Handa, P. F. Leadlay, I. C. Lennon, S. V. Ley and J. Staunton, Tetrahedron Lett., 1994, 35, 311.

C. J. Dutton, A. M. Hooper, P. F. Leadlay and J. Staunton, Tetrahedron Lett., 1994, 35, 327.

A. I. Scott, G. T. Philips and H. Kircheis, Bioorg. Chem., 1979, 1, 380.

F. Malpartida and D. Hopwood, Mol. Gen. Genet., 1986, 205, 63.

T. J. Simpson, Chem. Ind., 1995, 407.

D. A. Hopwood, F. Malpartida, H. M. Kieser, H. Ikeda, J. Duncan, I. Fujii,

B. A. M. Rudd, H. G. Floss and S. Omura, Nature, 1985, 314, 642. 
R. McDaniel, S. Ebert-Khosla, D. A. Hopwood and C. Khosla, Nature, 1995, 375, 549 .

R. McDaniel, S. Ebert-Khosla, D. A. Hopwood and C. Khosla, J. Am. Chem. Soc., 1995, 115, 11671.

C. M. Kao, G. Luo, L. Katz, D. E. Cane and C. Khosla, J. Am. Chem. Soc., $1994,116,11612$

C. W. Carreras, R. Pieper and C. Khosla, J. Am. Chem. Soc.,1996, 118, 5158.

J. Rohr, Angew. Chem., Int. Ed. Engl., 1995, 34, 881.

56 J. Cortes, S. F. Haydock, G. A. Roberts, D. J. Bevitt and P. F. Leadlay, Nature, 1990, 348, 176.

S. Donadio, M. J. Stavier, J. B. McAlpine, S. J. Swanson and L. Katz, Science, 1991, 252, 675.

C. M. Kao, G. Luo, L. Katz, D. E. Cane and C. Khosla, J. Am. Chem. Soc., $1994,116,11612$.

C. M. Kao, G. Luo, L. Katz, D. E. Cane and C. Khosla, J. Am. Chem. Soc., 1995, 117, 9105.

60 J.-F. Martin, Ann. Rev. Microbiol., 1977, 31, 13.

61 S. D. Rychnovsky, Chem. Rev., 1995, 95, 2021.

62 P. H. Harrison, H. Noguchi and J. C. Vederas, J. Am. Chem. Soc., 1986, 108, 3833 .

63 T. H. Sternberg, E. T. Wright and M. Oura, Antibiotics Annu., 1955-56, 566.

B. A. Steinberg, W. P. Jambar, L. O. Suydam, Antibiotics Annul., 1955-56, 574.

W. Gold, H. A. Stout, J. F. Pagano and R. Donovick, Antibiotics Annul., 
$1955-56,579$.

J. Vandeputte, J. L. Wadtel and E. T. Stiller, Antibiotics Annul., 1955-56, 587.

N. H. Georgopapadakou and T. J. Walsh, Science, 1994, 264, 371. $1975,28,317$.

H. A. B. Linke, W. Mechlinski and C. P. Schaffner, J. Antibiot., 1974, 27, 155.

N. Monji, W. Mechlinski, and C. P. Schaffner, J. Antibiot., 1976, 29, 435.

73 W. Mechlinski, C. P. Schaffner and P. Ganis, Tetrahedron Lett., 1970, 3873.

74 P. Ganis, G. Avitabile, W. Mechlinski and C. P. Schaffner, J. Am. Chem. Soc., 1971, 93, 4560.

75 K. C. Nicolaou, T. K. Chakraborty, Y. Ogawa, R. A. Daines, N. S. Simpkins and G. T. Furst, J. Am. Chem. Soc., 1988, 110, 4660.

76 K. C. Nicolaou, R. A. Daines, J. Uenishi, W. S. Li, D. P. Papahatjis and T.

K. Chakraborty, J. Am. Chem. Soc., 1988, 110, 4672.

77 K. C. Nicolaou, R. A. Daines, T. K. Chakraborty and Y. Ogawa, J. Am. Chem. Soc., 1988, 110, 4685.

78 K. C. Nicolaou, R. A. Daines, W. Ogawa and T. K. Chakraborty, J. Am. Chem. Soc., 1988, 110, 4696.

79 R. Thieringer and W.-H. Kunau, J. Biol. Chem., 1991, 266, 13110.

80 Ch. T. Buse and C. H. Heathcock, J. Am. Chem. Soc., 1977, 99, 8109.

81 G. Fráter, U. Müller and W. Günther, Tetrahedron, 1984, 40, 1269. 

Acta., 1981, 64, 2617. Soc., 1991, 113, 8024. and J. Lampe, J. Org. Chem., 1980, 45, 1066. Press, New York, 1984, vol. 3, p.111. C. H. Heathcock, M. C. Pirrung, S. H. Montgomery and J. E. Lampe, Tetrahedron, 1981, 37, 4087. Chem. Soc., 1973, 95, 3310.

H. Hiraua and S. Masamune, Tetrahedron Lett., 1979, 20, 2225.

S. Masamune, T. Sato, B.-M. Kim and T. A. Wollmann, J. Am. Chem. Soc, $1986,108,8279$. 40, 1381.

S. G. Davies, I. M. Dordor and P. Warner, J. Chem. Soc., Chem. Commun., $1984,956$. 

2127.

100 H. C. Hailes, S. Handa, P. F. Leadlay, I. C. Lennon, S. V. Ley and J. Staunton, Tetrahedron Lett., 1994, 35, 315.

101 D. A. Evans, M. D. Ennis and J. D. Mathre, J. Am. Chem. Soc., 1982, 104, 1737. 1123.

D. A. Evans, M. M. Morrissey and R. L. Dorrow, J. Am. Chem. Soc., 1985, $107,4346$. D. A. Evans, M. D. Ennis, T. Le, N. Mandel and G. Mandel, J. Am. Chem. Soc., 1984, 106, 1154.

D. A. Evans and J. R. Gage, Org. Syn., 1988, 68, 77.

A. Homer, Chem. Abst., 1940, 40, 4084.

M. S. Newman and A. Kutner, J. Am. Chem. Soc., 1951, 73, 4199.

D. A. Evans, D. J. Mathre and W. L. Scott, J. Org. Chem., 1985, 50, 1830.

111 S. H. Huno, A. Hirao, S. Nakahama and N. Yamazaki, J. Chem. Soc., Perkin Trans. 1, 1983, 1673.

112 G. A. Smith and R. E. Gawley, Org. Syn., 1985, 63, 136.

113 C. F. Lane, H. L. Myatt, J. Daniels and H. B. Hopps, J. Org. Chem., 1974, 39,3052 .

114 H. C. Brown, Y. M. Choi and S. Narasimhan, J. Org. Chem., 1982, 47, 3153. 
Collum, J. Am. Chem. Soc., 1991, 113, 5751.

T. Oishi and T. Nakata, Acc. Chem. Res., 1984, 17, 338.

130 Y. Ito, T. Katsuki and M. Yamaguchi, Tetrahedron Lett., 1985, 26, 4643. 3009. 

Structure, 3rd edn., Wiley, New York, 1985, p. 346. T. W. Greene and P. G. M. Wutts, Protecting Groups in Organic Synthesis, 2nd edn., Wiley, New York, 1991.

138 D. A. Evans, T. C. Britton and J. A. Ellman, Tetrahedron Lett., 1987, 28, 6141. J. M. Schwabb and J. B. Klassen, J. Am. Chem. Soc., 1984, 106, 7217. B. Neisses and W. Steglich, Angew. Chem., Int. Ed. Engl., 1978, 17, 522. H. Hailes, Ph.D. Thesis, University of Cambridge, 1991. K. F. Chater, Trends Genet., 1989, 5, 372. P. F. Stanbury, in Molecular Biology and Biotechnology, eds. J. M. Walker and E. B. Gingold, 3rd edn., The Royal Society of Chemistry, Cambridge, 1993.

R. K. Dart, Microbiology for the Analytical Chemist, The Royal Society of Chemistry, Cambridge, 1996. J. D. Bu'Lock, D. Hamilton, M. A. Hulme, A. J. Powell, D. Shepherd, H. M. Smalley and G. N. Smith, Can. J. Microbiol., 1965, 11, 765. M. E. Bushell, in Principles of Biotechnology, ed. A. Wiseman, Chapman and Hall, New York, 1988. A. H. Thomas, Analyst, 1976, 101, 321.

149 D. E. Cane, P. C. Prabhakaran, W. Tan and W. R. Ott, Tetrahedron Lett., $1991,32,5457$.

M. J. Rees, E. A. Cutmore, M. S. Verrall, in Separations for Biotechnology 3, ed. D. L. Pyle, The Royal Society of Chemistry, Cambridge, 1994, pp. $399-405$

A. Aszalos, A. Bax, N. Burlinson, P. Rollier and C. McNeal, J. Antibiot., $1985,38,1699$. 
152 R. C. Pandy and K. L. Rinehart Jnr., J. Antibiot., 1976, 29, 1035.

153 J. M. Brown and P. J. Sidebottom, Tetrahedron, 1981, 37, 1421.

154 P. Sowinski, J. Pawlak and E. Borowski, J. Magnetic Resonance, 1992, 30, 275

D. M. Ashworth and J. A. Robinson, J. Chem. Soc., Perkin Trans. 1, 1988, 1719.

156 G. R. Sood, D. M. Ashworth, A. A. Ajaz and J. A. Robinson, J. Chem. Soc., Perkin Trans. 1, 1988, 3183.

R. Thomas and D. J. Williams, J. Chem. Soc., Chem. Commun., 1983, 128.

Y. Doi, M. Kunioka, Y. Nakamura and K. Soga, J. Chem. Soc., Chem.

Commun., 1986, 1696.

159 J. C. Vederas, Nat. Prod. Rep., 1987, 4, 277.

160 D. S. Holmes, J. A. Sherringham, U. C. Dyer, S. T. Russell and J. A. Robinson, Helv. Chim. Acta, 1990, 73, 239.

J. Crawforth, Ph.D. Thesis, University of Leicester, 1996.

162

P. H. R. Harrison, Ph.D. Thesis, University of Alberta, 1987.

163

W. C. Still, M. Kahn and A. Mitra, J. Org. Chem., 1978, 43, 2923.

164 Aldrich Catalogue/Handbook of Fine Chemicals, 1996-1997, p. 907.

165 Aldrich Catalogue/Handbook of Fine Chemicals, 1996-1997, p. 83. 
Appendix $\mathbb{A}$ 
Table A1 Crystal data and structure refinement for 105.

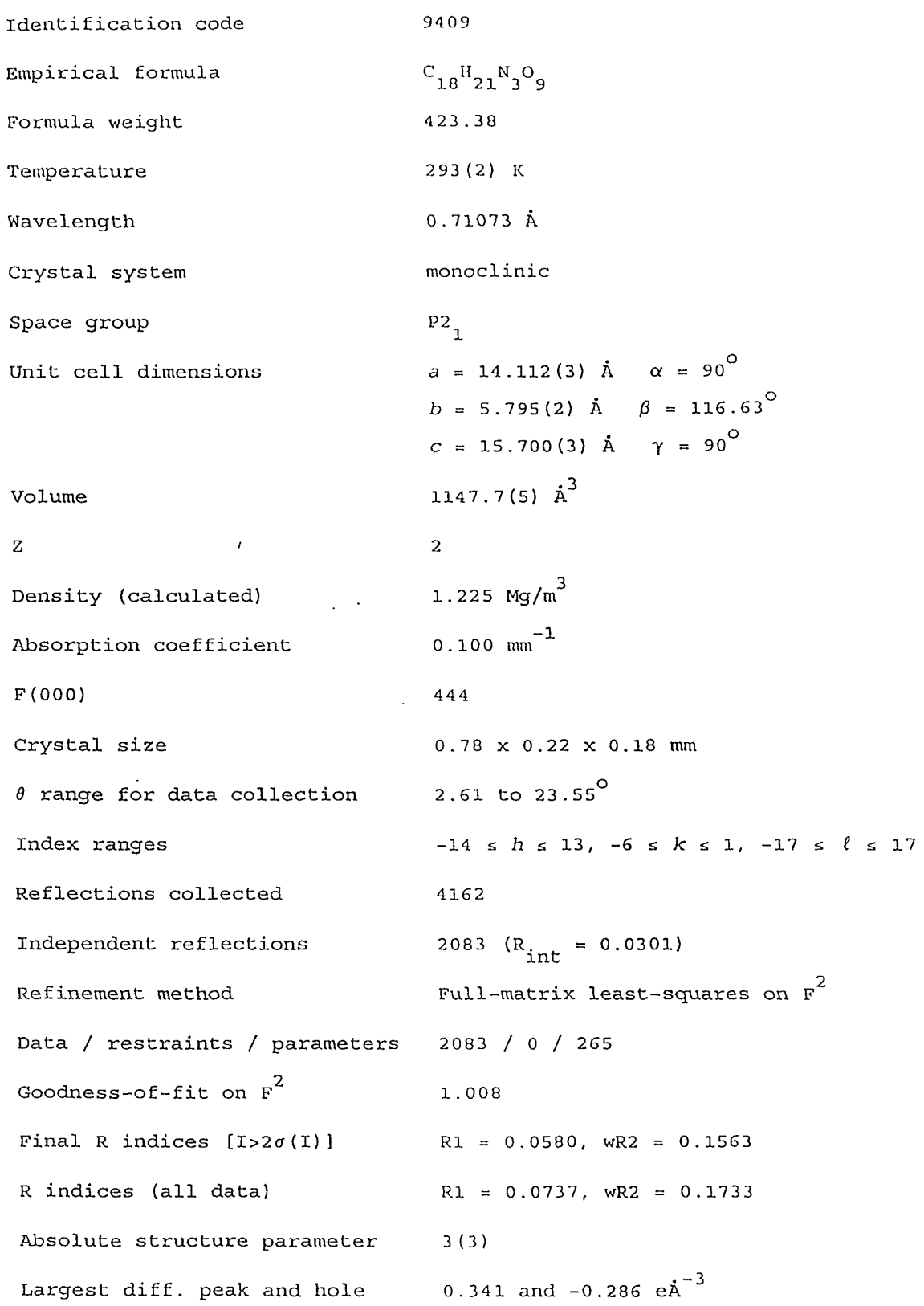


Table A2 Bond lengths $(\AA)$ and angles $\left(^{\circ}\right)$ for 105 .

\begin{tabular}{|c|c|c|c|}
\hline$O(1)-C(3)$ & $1.339(6)$ & $O(1)-C(1)$ & $1.439(8)$ \\
\hline$O(2)-C(3)$ & $1.198(6)$ & $O(3)-C(7)$ & 1. $221(7)$ \\
\hline$O(4)-C(12)$ & $1.298(8)$ & $O(4)-C(10)$ & $1.476(5)$ \\
\hline$O(5)-C(12)$ & $1.199(9)$ & $O(6)-N(2)$ & $1.206(6)$ \\
\hline$O(7)-N(2)$ & $1.227(6)$ & $O(8)-N(3)$ & $1.212(7)$ \\
\hline$O(9)-N(3)$ & $1.196(9)$ & $N(1)-C(3)$ & 1. $396(8)$ \\
\hline$N(1)-C(7)$ & $1.384(7)$ & $N(1)-C(2)$ & $1.474(6)$ \\
\hline$N(2)-C(15)$ & $1.479(9)$ & $N(3)-C(17)$ & $1.484(8)$ \\
\hline$C(1)-C(2)$ & $1.506(9)$ & $C(2)-C(4)$ & $1.531(7)$ \\
\hline$C(4)-C(6)$ & $1.456(11)$ & $C(4)-C(5)$ & $1.517(7)$ \\
\hline$C(7)-C(8)$ & $1.51 .6(7)$ & $C(8)-C(10)$ & $1.501(8)$ \\
\hline$C(8)-C(9)$ & $1.538(8)$ & $C(10)-C(11)$ & $1.514(12)$ \\
\hline$C(12)-C(13)$ & $1.498(7)$ & $C(13)-C(14)$ & 1. $373(9)$ \\
\hline$C(13)-C(18)$ & $3.384(8)$ & $C(14)-C(15)$ & $1.381(7)$ \\
\hline$C(15)-C(16)$ & $1.372(8)$ & $C(16)-C(17)$ & $1.353(9)$ \\
\hline$C(17)-C(18)$ & $1.390(7)$ & $C(19)-C(20)$ & $1.77(4)$ \\
\hline$C(19)-C(21) \# 1$ & $1.99(3)$ & $C(20)-C(21)$ & $2.14(4)$ \\
\hline$C(21)-C(19) \# 2$ & $1.99(3)$ & & \\
\hline$C(3)-O(1)-C(1)$ & $110.7(5)$ & $C(12)-O(4)-C(10)$ & $118.7(5)$ \\
\hline$C(3)-N(1)-C(7)$ & $127.6(4)$ & $C(3)-N(I)-C(2)$ & $111.2(4)$ \\
\hline$C(7)-N(1)-C(2)$ & $120.6(4)$ & $O(6)-N(2)-O(7)$ & $123.6(6)$ \\
\hline$O(6)-N(2)-C(15)$ & $118.7(5)$ & $O(7)-N(2)-C(15)$ & $117.7(6)$ \\
\hline$O(9)-N(3)-O(8)$ & $124.0(7)$ & $O(9)-N(3)-C(17)$ & $117.8(6)$ \\
\hline$O(8)-N(3)-C(17)$ & $118.2(7)$ & $O(1)-C(1)-C(2)$ & $107.0(4)$ \\
\hline$N(1)-C(2)-C(1)$ & $101.5(4)$ & $N(1)-C(2)-C(4)$ & $112.6(4)$ \\
\hline$C(I)-C(2)-C(4)$ & $114.5(5)$ & $O(2)-C(3)-O(1)$ & $122.4(6)$ \\
\hline$O(2)-C(3)-N(1)$ & $128.5(5)$ & $O(1)-C(3)-N(1)$ & $109.0(5)$ \\
\hline$C(6)-C(4)-C(5)$ & $111.7(6)$ & $C(6)-C(4)-C(2)$ & $123.7(6)$ \\
\hline$C(5)-C(4)-C(2)$ & $109.4(5)$ & $O(3)-C(7)-N(1)$ & $118.5(5)$ \\
\hline$O(3)-C(7)-C(8)$ & $121.4(5)$ & $N(1)-C(7)-C(8)$ & $120.1(5)$ \\
\hline$C(10)-C(8)-C(7)$ & $106.4(4)$ & $C(10)-C(8)-C(9)$ & $113.0(5)$ \\
\hline$C(7)-C(8)-C(9)$ & $107.9(5)$ & $O(4)-C(10)-C(8)$ & $107.4(4)$ \\
\hline$O(4)-C(10)-C(11)$ & $105.8(5)$ & $C(8)-C(10)-C(1 I)$ & $112.1(6)$ \\
\hline$O(5)-C(12)-O(4)$ & $124.8(6)$ & $O(5)-C(12)-C(13)$ & $122.2(6)$ \\
\hline$O(4)-C(12)-C(13)$ & $1.12 .9(6)$ & $C(14)-C(13)-C(18)$ & $120.9(5)$ \\
\hline$C(14)-C(13)-C(12)$ & $121.4(5)$ & $C(18)-C(13)-C(12)$ & $117.6(6)$ \\
\hline$C(13)-C(14)-C(15)$ & $119.0(5)$ & $C(16)-C(15)-C(14)$ & $122.0(6)$ \\
\hline$C(16)-C(15)-N(2)$ & $119.2(5)$ & $C(14)-C(15)-N(2)$ & $118.8(5)$ \\
\hline$C(15)-C(16)-C(17)$ & $117.1(5)$ & $C(16)-C(17)-C(18)$ & $124.0(5)$ \\
\hline$C(16)-C(17)-N(3)$ & $128.1(5)$ & $C(18)-C(17)-N(3)$ & $117.9(7)$ \\
\hline$C(13)-C(1.8)-C(17)$ & $116.9(6)$ & $C(20)-C(19)-C(21)$ \#I & $68(2)$ \\
\hline$C(19)-C(20)-C(21)$ & $164(2)$ & $C(20)-C(21)-C(19) \# 2$ & $68(2)$ \\
\hline
\end{tabular}

Symmetry transformations used to generate equivalent atoms:

$\# 1-x+1, y+1 / 2,-z+1 \quad \# 2-x+1, y-1 / 2,-z+1$ 